TECHNICAL REPORT NO. 3-753

\title{
TRAFFICABILITY CLASSIFICATION OF THAILAND SOILS
}

by

M. P. Meyer

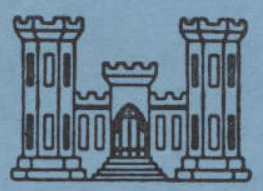

Yetz Reference Room

Civil Enginuering Department

B106 C. E. Building

University of Illinois

Uxbana, III1no18 61801

January 1967

Sponsored by

Advanced Research Projects Agency

Directorate of Remote Area Conflict

Service Agency

U. S. Army Materiel Command

Conducted by

U. S. Army Engineer Waterways Experiment Station CORPS OF ENGINEERS

Vicksburg, Mississippi

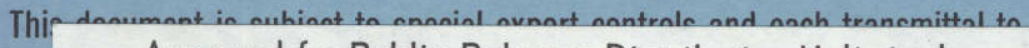

fort

Approved for Public Release; Distribution Unlimited

app 
Destroy this report when no longer needed. Do not return it to the originator.

The findings in this report are not to be construed as an official

Department of the Army position unless so designated by other authorized documents. 
TECHNICAL REPORT NO. 3-753

\section{TRAFFICABILITY CLASSIFICATION OF THAILAND SOILS}

by

M. P. Meyer

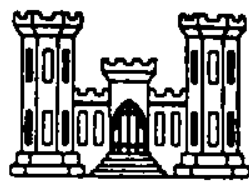

January 1967

Sponsored by

Advanced Research Projects Agency Directorate of Remote Area Conflict

Order No. 400

Service Agency

U. S. Army Materiel Command

Project No. I-V-0-2170I-A-046

Task 02

Conducted by

U. S. Army Engineer Waterways Experiment Station CORPS OF ENGINEERS

Vicksburg, Mississippi

ARMY.MRC VICKSBURG. MISS.

This Approved for Public Release; Distribution Unlimited

fore

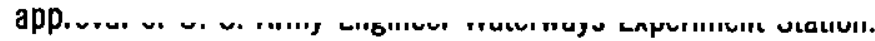



FOREWORD

The study reported herein constitutes a portion of the Mobility Environmental Research Study (MERS), sponsored by the Office, Secretary of Defense, Advanced Research Projects Agency (ARPA), Directorate of Remote Area Conflict, for which the U. S. Army Engineer Waterways Experiment Station (WES) is the prime contractor, and the U. S. Army Materiel Command (AMC) is the service agent. The broad mission of Project MERS is to determine the effects of the various features of physical environment on the performance of cross-country, ground-contact vehicles and to provide therefrom data which can be used to improve both the design and employment of such vehicles. One criterion of the project is that the data be interpretable in terms of vehicle requirements for Southeast Asia. Most of the funds employed for this study were allocated to WES through AMC under ARPA Order No. 400. The remaining funds were provided by the Directorate of Research and Development, AMC, as part of Department of the Army Project No. 1-V-0-21701-A-046, "Trafficability and Mobility Research," Task 1-V-021701-A-046-02, "Surface Mobility."

The study was performed by personnel of WES during the period August 1964-May 1966. The study was assigned to the Army Mobility Research Branch (AMRB), Mobility and Environmental (M\&E) Division. Mr. M. P. Meyer had the primary responsibility for the general conduct of the study including the preparation of this report. Mr. J. G. Kennedy programmed the data for computer analysis. Mr. G. T. Ellis compiled the data and assisted in the analysis. Mr. C. A. Blackmon wrote the appendix. Others assisting in the study include Messrs. S. M. Hodge, J. E. Lee, and H. D. Molthan. All phases of the study were under the direct supervision of Mr. E. S. Rush, Chief of the Trafficability Section, AMRB, and the general supervision of 
Dr. D. R. Freitag, Chief, AMRB; Mr. A. A. Rula, Chief, MERS Branch; Messrs. W. G. Shockley and S. J. Knight, Chief and Assistant Chief, respectively, M\&E Division; and Mr. W. J. Turnbull, Technical Assistant for Soils and Environmental Engineering.

Directors of the WES during the conduct of this study and the preparation of this report were Col. Alex G. Sutton, Jr., CE, and Col. John R. Oswalt, Jr., CE. Technical Director was Mr. J. B. Tiffany. 
CONTENTS

FOREWORD . . . . . . . . . . . . . . . . . . . . . . .

$\underline{\text { Page }}$

SUMMARY.

iii

PART I:

Background . . . . . . . . . . . . . . . . . . .

Purpose. . . . . . . . . . . . . . . . . . .

Scope. . . . . . . . . . . . . . . . . . . .

General Approach . . . . . . . . . . . . . . .

vii

PART II: TRAFFICABILITY FACTORS

Soil Bearing and Traction Capacities . . . . . . . . . . . Methods of Measuring and Evaluating Soil Trafficability. . . . Soil Moisture. . . . . . . . . . . . . . . . . . . Slope.

PART III: ANALYSIS OF DATA. . . . . . . . . . . . . . . . . 16

Basic Data . . . . . . . . . . . . . . . 16

Method of Computation. . . . . . . . . . . . . . 18

Mean and Standard Deviation Values of Soil Properties. . . . . 18

Cumulative Frequency Analysis of Rating Cone Index . . . . . . 23

PART IV: SOIL TRAFFICABILITY CLASSIFICATION SCHEME AND RELATED

STUDIES . . . . . . . . . . . . . . . . . . . 26

Vehicle Categories . . . . . . . . . . . . . . . 26

Soil Trafficability Classification Scheme, Level Terrain . • . 27

Probability of Vehicle "Go" on Level and Sloping Terrain . . . 29

Application of Information for Estimating Trafficability

Conditions . . . . . . . . . . . . . . . . 31

Estimation of Percentage of Area Trafficable . . . . . . . 34

PART V: CONCLUSIONS AID RECOMMENDATIONS . . . . . . . . . . . . 36

Conclusions. . . . . . . . . . . . . . . . 36

Recommendations. . . . . . . . . . . . . . . 38 


\section{CONTENTS}

TABLES $1-11$

PLATES 1-10

APPENDIX A: SOURCES OF DATA AND DETAILED PROCEDURES USED TO OBTAIN DATA . . . . . . . . . . . . . . . . . . . . . . AI

Preliminary Survey Study . . . . . . . . . . . . . A2

Trafficability Classification Study . . . . . . . . . . A3

Surface Composition Study . . . . . . . . . . . . A6

Soil Moisture-Strength Study . . . . . . . . . . . . . . AT

U. S. Army Cold Regions Research and Engineering Laboratory

(CRREL) Airphoto Pattern Study . . . . . . . . . . . AlO

Terrain-Vehicle Tests . . . . . . . . . . . . . All

TABLES AI-A6 
SUMMARY

The study reported herein consisted of a statistical analysis of the principal factors that influence soil trafficability and the application of the analysis to the development of a scheme for classifying soils under generally wet conditions in Thailand. The scheme is essentially a listing of soil types (in terms of the Unified Soil Classification System and the U. S. Department of Agriculture textural classification system) in decreasing order of median rating cone index. Means and ranges are given for each soil type in high- and low-topography positions for average and highest soil-moisture conditions during the wet season. The probability of successful negotiation of a soil type by military vehicles can be ascertained by comparing vehicle cone indexes with the frequency distribution of rating cone indexes for the soils. Results of the studies performed in the development of the trafficability scheme are sunmarized as follows:

a. The probability of "go" for a given vehicle over a given soil. type is higher on high topographic positions than on low topographic positions; on low topographic positions the probability of "go" is lowest during times of maximum soilmoisture conditions. For a given topography-moisture condition the probability of "go" decreases for USCS soils in the following order: clean, coarse-grained soils, coarse-grained soils with fines, fine-grained soils, and organic soils.

b. Soils in Thailand have slight.ly higher strengths under wettest soil-moisture conditions and slightly lower strengths under average soil-moisture conditions during the wet season than do soils in humid-temperate areas of the United States. 

PART I: INTRODUCTION

Background

1. The study reported herein is a part of an extensive investigation conducted to develop techniques and procedures for determining off-road soil conditions in Thailand and a graphic means of presenting these conditions which will show the relations between vehicle mobility, soil type and moisture content, and slope. This study consisted of a statistical analysis of the principal factors that influence soil trafficability and the application of the analysis in developing a scheme for classifying Thailand soils under generally wet conditions. The scheme presented herein is essentially the same as that reported in a previous WES publication. ${ }^{1}$ The major differences are that this scheme is applied to a more restricted area, and some refinement of analytical procedures has been made.

\section{Purpose}

2. The purpose of this study was to develop a scheme for classifying the trafficability of Thailand soils in the wet season based on identification of the soils in terms of the Unified Soil Classification System (USCS) and the U. S. Department of Agriculture (USDA) textural classification system, general topographic data, and two general levels of soilmoisture content.

$\underline{\text { Scope }}$

3. Trafficability data collected at 846 sites in Thailand on coarsegrained soils with fines and fine-grained soils of the 0 - to 6- and 6- to 12-in. soil layers were statistically analyzed in the development of a trafficability classification scheme. In 1964 data from 238 sites were collected specifically for this study. Other data used were collected in various Mobility Environmental Research Study (MERS) programs including: 
a preliminary survey of environmental factors affecting ground mobility in Thailand, ${ }^{2}$ performed in 1962; the study of a quantitative method for describing terrain for ground mobility, surface composition, ${ }^{3}$ performed in 1964-1965; a study of soil moisture-strength characteristics of selected soils in Thailand, 4 performed in 1963-1965; a study of selected airphoto patterns of terrain features, ${ }^{5}$ performed in 1964-1965 by the U. S. Army Cold Regions Research and Engineering Laboratory (CRREL); and tests to develop an analytical model for predicting cross-country vehicle performance, 6 performed in 1965. Most of these data came from the Chiang Mai, Nakhon Sawan, Khon Kaen, Lop Buri, Chanthaburi, and Pran Buri study areas. Analyses were made of cone index, remolding index, rating cone index, surface shear strength, moisture content, density, and specific gravity of soils of low and high topography identified according to the USCS and the USDA textural classification system.

\section{General Approach}

4. A soil trafficability classification scheme, if it is to be practicable, must first name or identify the soils according to some recognized system of soil classification, then establish trafficability limits for each soil type, and finally, if feasible, collect the various soil types into a small number of groups, each exhibiting a discrete trafficability behavior. The ideal scheme would be one that provides for consideration and evaluation of all aspects of the environment (pedologic, geologic, hyarologic, physiographic, climatic, and vegetative) that affect the trafficability of the soil. The scheme reported herein considers the soil type under very general space and time conditions in a tropical climate. Further refinement according to the environmental characteristics mentioned previously must await the collection of additional data and further analysis.

5. Because their trafficability behavior is not materially affected by moisture content, clean sands and gravel have been given a distinct place in the soil trafficability classification scheme and have been excluded from the various statistical analyses that are presented in this report. 
6. The soil trafficability classification scheme presented in this report may be considered a composite classification scheme because it uses two well-known systems of soil identification and is based on two moisture levels. The two soil classification systems used are the $\operatorname{USCS}^{7}$ (fig. I) and the USDA soil textural classification system $^{8}$ (fig. 2). The USCS employs soil texture, plasticity, and organic content to name or type soils, whereas the USDA system is based solely on grain size distribution. Because the USCS characterizes soils on the basis of their engineering behavior, it is considered to be more applicable to the development of a soil trafficability classification scheme than the USDA system. However, since many areas in Thailand and other areas in Southeast Asia are mapped in USDA terms, it was also considered desirable to develop a scheme in USDA terms. 


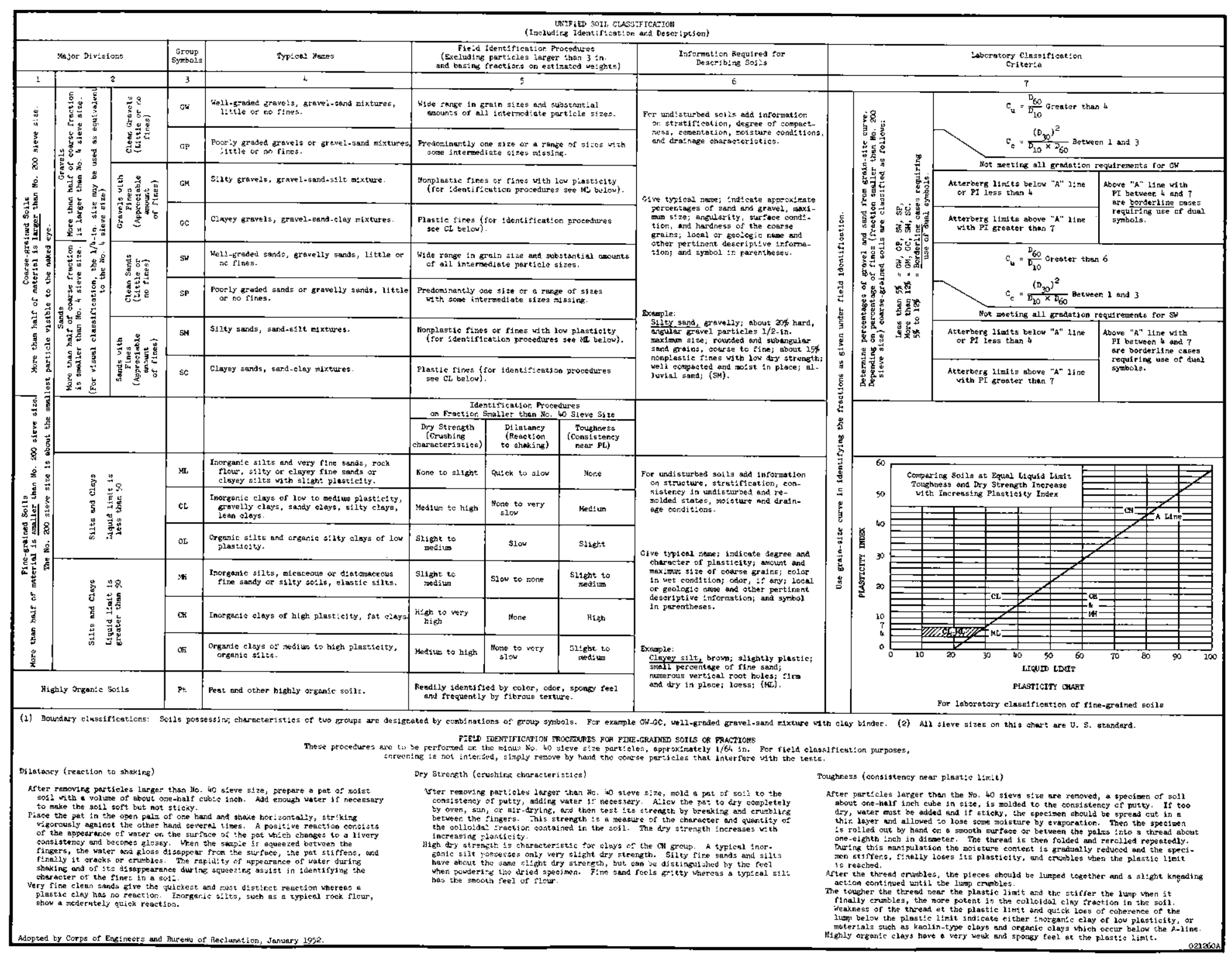

Fig. 1. Unified Soil 'Classification System 


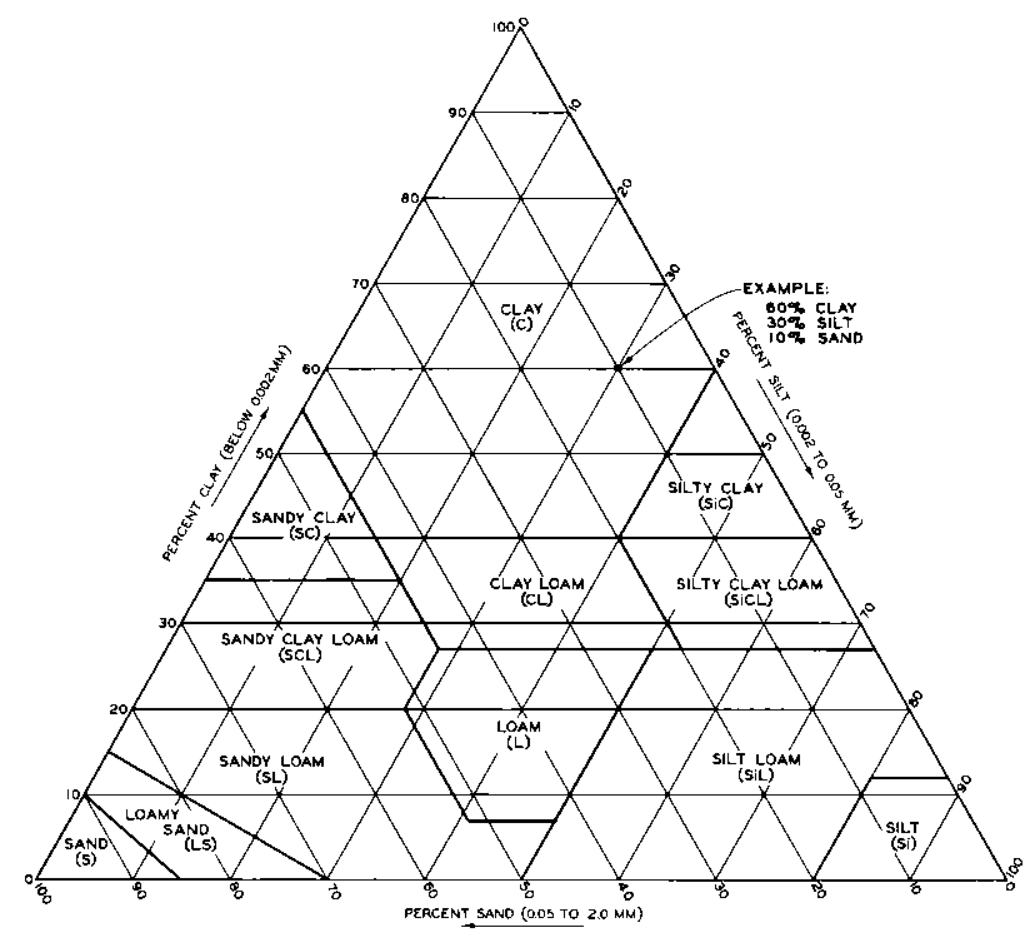

Fig. 2. USDA soil textural classification system 
7. Trafficability is defined as the capacity of a soil to withstand traffic by vehicles. It is an important aspect of cross-country movement which may be defined as the ability of terrain to permit the movement of vehicles. The factors that influence cross-country movement are numerous. They include not only the many variables which combine to determine the strength and other physical properties of soils, but also slope and other natural obstacles such as drainageways, scarps, vegetation, and microrelief features, as well as man-made obstacles such as railroad embankments, canals, paddy dikes, etc. The investigation reported herein deals mainly with the trafficability of soils. However, the effects of slope are also considered.

\section{Soil Bearing and Traction Capacities}

8. Bearing and traction capacities are primarily functions of strength (or shearing resistance) of a soil. Bearing capacity is the ability of a soil to support a vehicle without undue sinkage; traction capacity is the ability of a soil to provide sufficient resistance between the propulsion element of a vehicle and the soil for the necessary thrust to move the vehicle forward. The trafficability of a soil is considered adequate for a given vehicle if the soil has sufficient bearing capacity to support the vehicle and sufficient traction capacity to enable the vehicle to develop the forward thrust necessary to overcome its rolling resistance.

\section{Methods of Measuring and Evaluating Soil Trafficability}

9. The soil strength measurements used in the WES system for predicting vehicle performance were used in developing the soil trafficability classification scheme discussed in this report. It has been demonstrated that the effect of soil on the performance of vehicles in terms of "go"*

* In this report "go" means that 50 vehicles can pass in straight-line traffic or one vehicle can execute severe maneuvers without becoming immobilized. 
and "no go," slope-climbing ability, drawbar pull, and force required to tow the vehicle can be predicted with reasonable accuracy if the mass and surface soil strengths are not vastly different. If the mass soil strength is high and the surface is wet or consists of a thin, soft soil layer, the vehicle will sink very little, but forward motion may be denied because of loss of traction. Present methods for predicting the performance of vehicles on such soils are not entirely satisfactory. Previous investigations have also shown that the change in strength of a soil which will be developed under vehicular traffic differs significantly for fine-grained and coarse-grained soils; therefore, the measurement and evaluation methods differ somewhat. These differences are discussed in the following paragraphs. Soil strength measurements used for determining trafficability

10. Fine-grained soils and sands with fines, poorly drained. In fine-grained soils and in sands with fines, poorly drained, cone index (CI) and remolding index (RI) measurements are necessary to define soil trafficability. The CI provides an index of the in-situ or undisturbed shear strength of the soil prior to vehicular traffic. It, by itself, is inadequate for predicting the soil strength after repeated traffic by a vehicle because repetitive traffic invariably remolds the soil, thus altering its strength. The probable effect of vehicular traffic on soil strength is obtained from the RI, which indicates the direction and magnitude of the strength change that can be anticipated under vehicular traffic. An RI less than 1.00 denotes a strength loss as a result of remolding; an RI greater than 1.00 indicates a gain in strength. For example, a wet silt may retain only $25 \%$ of its undisturbed strength once it is subjected to repetitive vehicular traffic.

11. The trafficability of fine-grained soils and sands with fines, poorly drained, is therefore defined in terms of a value called the rating cone index (RCI), which is the product of the CI and the RI for the same soil layer. In general, the soil layer between the 6- and 12-in. depths is critical for most military vehicles operating in such soils. However, the depth of the critical layer varies with the strength profile of the soil and the vehicle type and weight. 9,10 
12. Various instruments are currently being tested to determine their utility for predicting surface traction for soil trafficability purposes. One instrument (sheargraph) used in this study provides a measure of the ultimate cohesion and ultimate angle of internal friction for soil-to-soil and rubber-to-soil shear failures. In this study a normal load of 10 psi was arbitrarily selected as a constant in determining the surface shear strength of a soil. The equipment and procedures used in taking sheargraph measurements and in reducing and evaluating the data are described in references 11 and 12 .

13. Coarse-grained soils. For coarse-grained soils or clean sands, CI measurements alone are adequate to quantify trafficability. Usually, the strength of clean sands is not altered significantly by changes in moisture content. Clean sands possess adequate strength to support vehicles without critical sinkage. In clean sands the first pass is the most critical, and subsequent passes are made with less difficulty. The 0- to 6-in. layer is considered the critical layer for most military vehicles. Evaluation of soil trafficability

14. Fine-grained soils and sands with fines, poorly drained. The ability of a given vehicle to complete 40 to 50 passes traveling in a straight-line path over a level area or to execute severe maneuvers in fine-grained soils or sands with fines, poorly drained, is assured if the RCI of the soil in the critical layer in that area is equal to or greater than the vehicle cone index (VCI) assigned to that vehicle. In general, an RCI equal to 50\% of the VCI indicates sufficient soil strength to permit one or two straight-line passes of the vehicle. ${ }^{13}$ If the RCI is greater than the VCI of a given vehicle, the additional traction resulting from the excess soil strength can be used to accelerate the vehicle, negotiate slopes, or tow a load.

15. The VCI's for most military vehicles are tabulated in several publications. 9,10 The referenced publications also contain formulas for computing mobility indexes, means of relating these indexes to VCI's, and the relation of drawbar pull, slope, and force required to tow the vehicle to soil strength.

16. Coarse-grained soils. Studies being conducted on clean sands 
have not yet progressed to the point of quantifying trafficability. Results thus far indicate that tracked vehicles usually experience little or no difficulty traversing level, clean-sand areas. The effect of soil strength on vehicle performance (in terms of drawbar pull and slope-climbing ability) of a given tracked vehicle is small; however, a significant difference in performance exists among vehicles having different types of track systems. A wide range in wheeled-vehicle performance occurs as a result of changes in tire pressure, number of tires, and tire size.

\section{Soil Moisture}

17. The principal factor influencing the strength of a given soil is its moisture content. Any soil in a comparatively dry state may be trafficable to all military vehicles; but at high moisture content, its strength and consequently its trafficability may be such that only certain vehicles can pass. It is apparent that moisture conditions must be taken into account in any evaluation of the trafficability of soils and, further, that soils must be at similar or equivalent conditions of moisture in order that they can be rated fairly in comparison with each other.

18. Moisture is added to the soil through precipitation, a rising water table, flooding, or irrigation. Moisture is generally depleted from the soil by runoff, gravitational percolation, evaporation, or transpiration through plants. The rate and magnitude of moisture gain or loss and the capacity of the soil to hold water are controlled primarily by the soil and by site characteristics that determine the porosity and permeability of the soil. These characteristics, for the most part, are influenced by the plastic, organic, and textural properties of the soil that are defined in terms of the USCS and the USDA soil classification system. Climate and season

19. Climate must be considered in any type of soil-moisture analysis. The principal elements of climate consist of precipitation, temperature, atmospheric humidity and pressure, and wind velocity. Of these, precipitation and temperature are the two most important factors controlling the gain and loss of soil moisture. Similar soils within a specific climatic 
area wi.ll have qualitatively similar seasonal soil-moisture conditions; and conversely, similar soils of different climates will have dissimilar seasonal soil-moisture regimes. Soils in hot, humid climatic areas, for example, generally approach minimum moisture levels more rapidly than soils in cool, humid climatic areas because of higher rates of evapotranspiration. 20. For purposes of this study, a wet season and a dry season are considered, based on the qualitative moisture conditions of the soil. The wet season is defined as the period of the year when generally high soilmoisture contents prevail; it corresponds to the period of maximum precipitation. The dry season is defined as the period of generalty low soilmoisture contents, although maximum moisture contents may occur for short periods inmediately after several days of heavy rain.

21. Soil-moisture studies conducted at specific sites in various sections of Thailand for continuous periods of almost two years have been used to refine a system for predicting the effects of meteorological factors on the trafficability of soils. The studies show, among other things, that the top $12 \mathrm{in.}$ or so of soil attain relatively high moisture contents during the monsoon season beginning in May or June and continuing through October or November. The distribution of high-rainfall months wherein the rainfall exceeds $100 \mathrm{~mm}$ (3.94 in.) a month is recorded in the following tabulation for nine weather stations in Thailand that are located in areas that include most of the study sites.

\begin{tabular}{|c|c|c|c|c|c|c|c|c|c|c|c|c|c|}
\hline Loca & $\begin{array}{l}\text { Years } \\
\text { of }\end{array}$ & & $\mathrm{Pe}$ & ent 0 & $\begin{array}{l}I 16 \\
1\end{array}$ & $\mathrm{~s}$ & $\begin{array}{l}\operatorname{Recc} \\
3.94\end{array}$ & $\begin{array}{l}\text { d with } \\
\text { in.) } p\end{array}$ & $\operatorname{Rai}$ & $\begin{array}{l}\text { fall } \\
\text { nth }\end{array}$ & ee & & \\
\hline Weathe & Record & $\overline{\mathrm{Jan}}$ & Feb & Mar & $\underline{\text { Apr }}$ & May & June & JuIy & Aug & Sept & Oct & Nov & Dec \\
\hline Chiang Mai & $19-1 / 2$ & 0 & 0 & 0 & 20 & 80 & 75 & 89 & 100 & 100 & 63 & 11 & 0 \\
\hline Khon Kaen & 17 & 0 & 0 & 12 & 35 & 100 & 76 & 76 & 100 & 100 & 35 & 0 & 0 \\
\hline Nakhon Sawan & 15 & 0 & 7 & 0 & 13 & 53 & 53 & 67 & 93 & 100 & 73 & 0 & 0 \\
\hline Lop Buri & $17-1 / 2$ & 0 & 0 & 24 & 29 & 71 & 82 & 94 & 89 & 100 & 61 & 6 & 0 \\
\hline Bangkok & $23-2 / 3$ & 0 & 4 & 4 & 35 & 87 & 87 & 79 & 92 & 100 & 96 & 17 & 0 \\
\hline Chanthaburi & 23 & 4 & 9 & 26 & 70 & 100 & 100 & 100 & 100 & 100 & 96 & 26 & 0 \\
\hline $\begin{array}{l}\text { Sattahip } \\
\text { (Chanthaburi Area) }\end{array}$ & 23 & 0 & 17 & 17 & 48 & 74 & 26 & 30 & 35 & 87 & 96 & 52 & 4 \\
\hline $\begin{array}{l}\text { Hua Hin (Pran Buri } \\
\text { Area) }\end{array}$ & $20-1 / 3$ & 5 & 0 & 0 & 15 & 55 & 40 & 25 & 40 & 67 & 81 & 43 & 0 \\
\hline $\begin{array}{l}\text { Songkhla (Hat Yai } \\
\text { Area) }\end{array}$ & $22-2 / 3$ & 61 & 17 & 22 & 43 & 57 & 57 & 45 & 33 & 55 & 96 & 100 & 91 \\
\hline
\end{tabular}


22. In order to estimate the trafficability of a site more accurately, consideration must be given not only to its soil type but also to its topographic position and its general relative moisture-content level. From a study of the data available, certain arbitrary "space" and "time" factors have been designated that are considered essential for optimum accuracy in estimating trafficability on the basis of existing knowledge and available data. Additional data and further study may produce more explicit criteria for estimating the trafficability at a site. However, at the present time, two space factors (low and high topography) and two time factors (wet-season and high-moisture conditions) will be used. These are illustrated in fig. 3 and explained in the following paragraphs.

23. Space factors. The depth to the water table has been found to be a significant factor in determining how wet a site may become. Sites which have a water table within 4 ft of the surface become wetter in the top foot than do sites with the water table below the top $4 \mathrm{ft}$, even though all other conditions appear to be the same.

a. Low topography. A site of low topography is one at which a water table is known to exist within 4 ft of the surface, perennially or at some time during the year. Such sites usually occur as bottomlands, lower terraces, depressions, or bottoms of slopes, or occasionally as upland flats associated with impervious subsurface layers or pans. They are generally characterized by poor to fair external drainage and moderately poor to very poor internal drainage. If the water table is actually observed at depths of less than 4 ft from the surface at a site at least once, the site automatically qualifies as a low-topography site. If observed data on water-table depth are not available, sites which appear, from observation, likely to have high water tables on the basis of their topographic position, drainage characteristics, proximity to surface water bodies, or soil coloring (gray or blue mottled soils usually indicate the presence of a consistent water table) are judged to be lowtopography sites.

b. High topography. Sites of high topography have water tables at depths greater than 4 ft from the surface at all times. These sites are characterized by soils with no impervious layers or pans and with moderate to good internal and external drainage. They are usually located on ridges or upper 


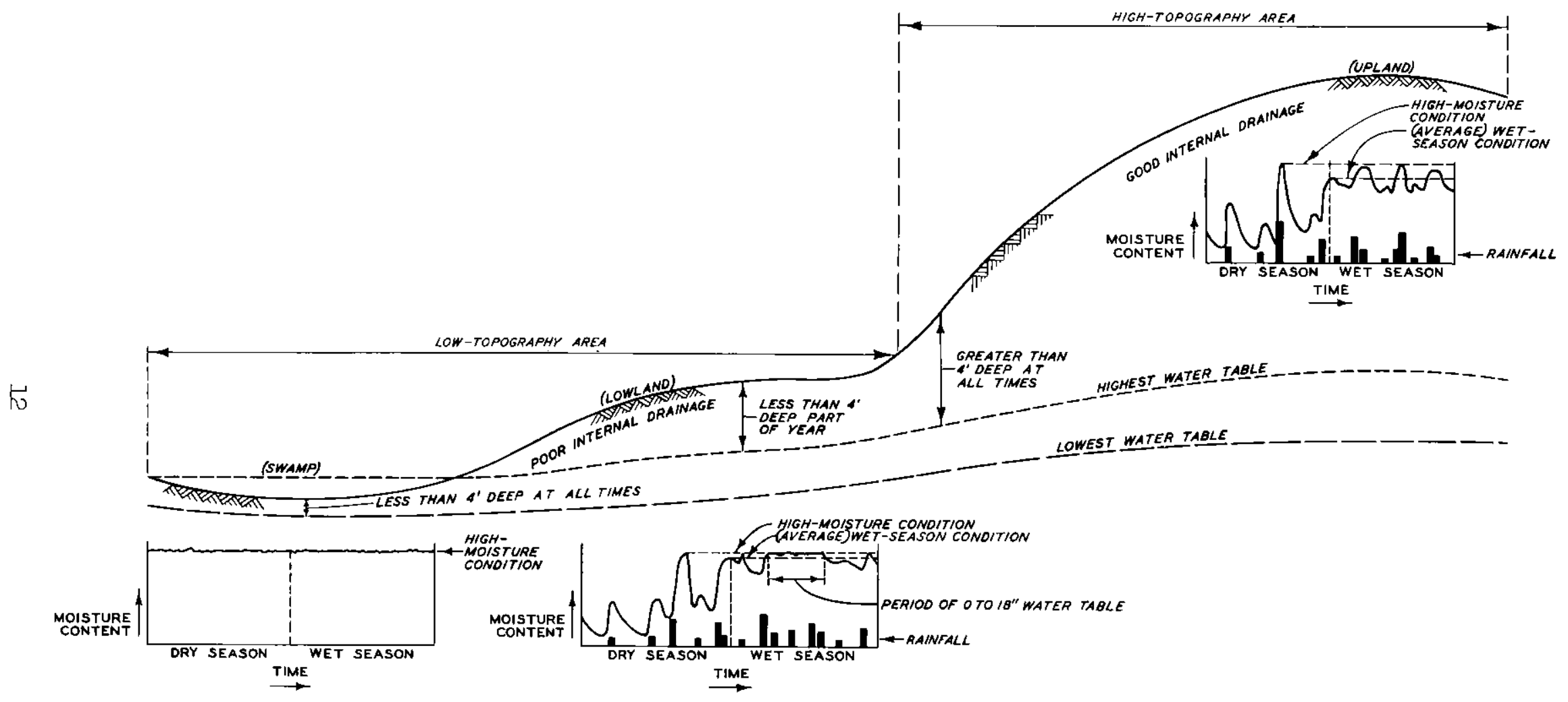

Fig. 3. Profile of a typical area showing various topography-moisture conditions during year 
slopes. If information on the water table is not available, it is usually possible to determine whether a site is one of high topography through a study of the topographic position and other environmental data available.

24. Time factors. While for this study it would have been desirable to have examined the means and ranges of pertinent soil values measured at a time when the moisture content was at rigorous reference levels, such as field maximum or field capacity, this was not feasible because only a few sites (the prediction-development sites) were known to have been tested when the moisture content was at these levels. In order to realize the benefit of values derived from large masses of data, less rigorous moisture levels, wet-season and high-moisture conditions, were selected. These conditions, or time factors as they are called in this report, are discussed in the following subparagraphs.

a. Wet-season condition. The wet-season condition is intended to represent the average moisture condition prevailing in soils during the wet season. Data from some of the drier sites were not utilized because the soil was too firm to obtain the necessary data for a determination of rating cone index. Exclusion of these data tended to bias the averages toward wetter-than-average conditions.

b. High-moisture condition. The high-moisture condition represents the worst trafficability condition that can occur at sites that undergo seasonal changes. Marshes, bogs, swamps, and other perennially wet, soft, spongy areas are prime examples of low-topography areas under a high-moisture condition at all times. Low-lying areas with fluctuating water tables and upland areas with seasonal perched water tables are typical examples of low-topography areas where a highmoisture condition occurs intermittently. Low- and hightopography areas that have been subjected to moderate or heavy rainfall are normally under a high-moisture condition during and immediately following rain periods. In this study a high-moisture condition at high-topography sites could not be identified from the collected data. Consequently, an analysis was not made for this topographymoisture condition and the classification scheme does not include data for this category. Only one set of highmoisture data (cone index, remolding index, rating cone index, sheargraph, and moisture content) was used in the analysis for a given low-topography site. At sites where highmoisture data were collected on more than one day, the set of data selected was for the day of lowest rating cone index. The moisture content for this day was usually, but not 
necessarily, the highest recorded at the site. In analyzing the data, a high-moisture condition was considered to have been prevalent at a low-topography site when it was known that the water table was within the top 18 in. of soil. (This 18-in. criterion is based on studies in the United States 14 that show that the strength of a soil decreases at a logarithmic rate with a decrease in depth to the water table and a relatively small rate of change of strength per unit change in depth to the water table when the water table is above a depth of $18 \mathrm{in.})$

\section{Slope}

25. Vehicles that can traverse certain soils on level surfaces often become immobilized when climbing slopes on similar soils. These immobilizations can be attributed primarily to a downhill force, a function of the vehicle's weight and the angle of slope, which opposes the vehicle's forward thrust. In this report slope is expressed in terms of percent (vertical rise divided by horizontal distance, multiplied by 100).

\section{Slope index}

26. The adverse effect of slope on vehicle performance can be expressed by an increase in rating cone index requirements above the vehicle's requirements for level terrain. These excess RCI points, called slope index, may be added to the vehicle cone index and the determination of "go" or "no go" is made by comparing this value with the measured RCI. Detailed procedures are available for determining slope effects and for estimating the maximum slopes negotiable by various velicle types.9,10 Three slope index values, one for tracked vehicles with grousers longer than $1-1 / 2$ in., another for tracked vehicles with grousers shorter than $1-1 / 2$ in., and the third for wheeled vehicles, can be obtained for a given slope from the three respective curves shown in plate 1 . If, for example, the slope is $30 \%$, the slope indexes for the three vehicle classes would be 13, 15, and 20, respectively.

Effective rating cone index (ERCI)

27. The ERCI is a combined soil strength-slope value which rates the trafficability of a sloping soi.l. The index is computed by subtracting the slope index from the rating cone index. For example, if the RCI of a soil 
is determined to be 50 and the slope is $30 \%$, the ERCI would be 37 (50 minus 13) for tracked vehicles with grousers longer than $1-1 / 2$ in.; 35 (50 minus 15) for tracked vehicles with grousers shorter than 1-1/2 in.; and 30 (50 minus 20) for wheeled vehicles. The determination of "go" or "no go" on sloping terrain is based on a comparison of the vehicle cone index with the ERCI for the vehicle class. If the VCI is greater than the ERCI, vehicles of this type will not be able to climb the slope; if the VCI is less than the ERCI, the slope is considered negotiable. The ERCI can also be applied and, if desired, mapped in regard to level terrain. In this case, the slope index is zero for all vehicle classes and the ERCI is equal to the RCI of the soil. 
28. The data were classified and analyzed in terms of both USCS and USDA soil types under a high-topography, wet-season condition, a lowtopography, wet-season condition, and a low-topography, high-moisture condition. The following studies were conducted:

a. A determination of means and standard deviations of cone index, remolding index, rating cone index, moisture content, dry density, and specific gravity for the 6- to 12-in. soil layer; cone index and moisture content for the 0- to 6-in. soil layer; and sheargraph shear strength for surface soils.

b. A cumulative frequency analysis of rating cone index for the 6- to 12-in. soil layer for each USCS and USDA soil type and for all soils.

\section{Basic Data}

29. The data used in these analyses were obtained from 846 sites, 701 of which were located in six MERS study areas, including 103 sites in Chiang Mai, 117 in Khon Kaen, 77 in Nakhon Sawan, 160 in Lop Buri, 182 in Chanthaburi, and 62 in Pran Buri. The remainder of the test sites were located in other sections of Thailand. The general locations of the sites are shown in fig. 4. The data were derived from six different test programs conducted for MERS during the period June 1962 through October 1965. The number of sites from each program which provided data for this study and for each analysis is shown in the following tabulation. The procedures

Number of sites

Mean and standard deviation

Wet-season condition

CT, 0.6 in.

CI, 6-12 in.

$\mathrm{RI}$ and $\mathrm{RCI}$, 6-12 in.

Sheargraph shear strength

Moisture content, $0-6$ in.

Moisture content, 6-12 in.

Dry density, 6-12 in.

Prelim-
inary
Survey

Traffic-

Class face

- Compo-

165

238

224

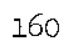

157

91

$1 \overline{45}$

130

116

\section{8}

238

193

227

238

238

193

(Continued)

\begin{tabular}{rrrrr}
224 & 75 & 105 & 23 & 825 \\
224 & 75 & 105 & 23 & 822 \\
146 & 70 & 69 & 14 & 583 \\
197 & 50 & -- & 22 & 496 \\
106 & 75 & 105 & 22 & 691 \\
105 & 75 & 104 & 20 & 672 \\
29 & 75 & -- & 10 & 423 \\
\hline
\end{tabular}

* Also used in analysis of cumulative frequency. 


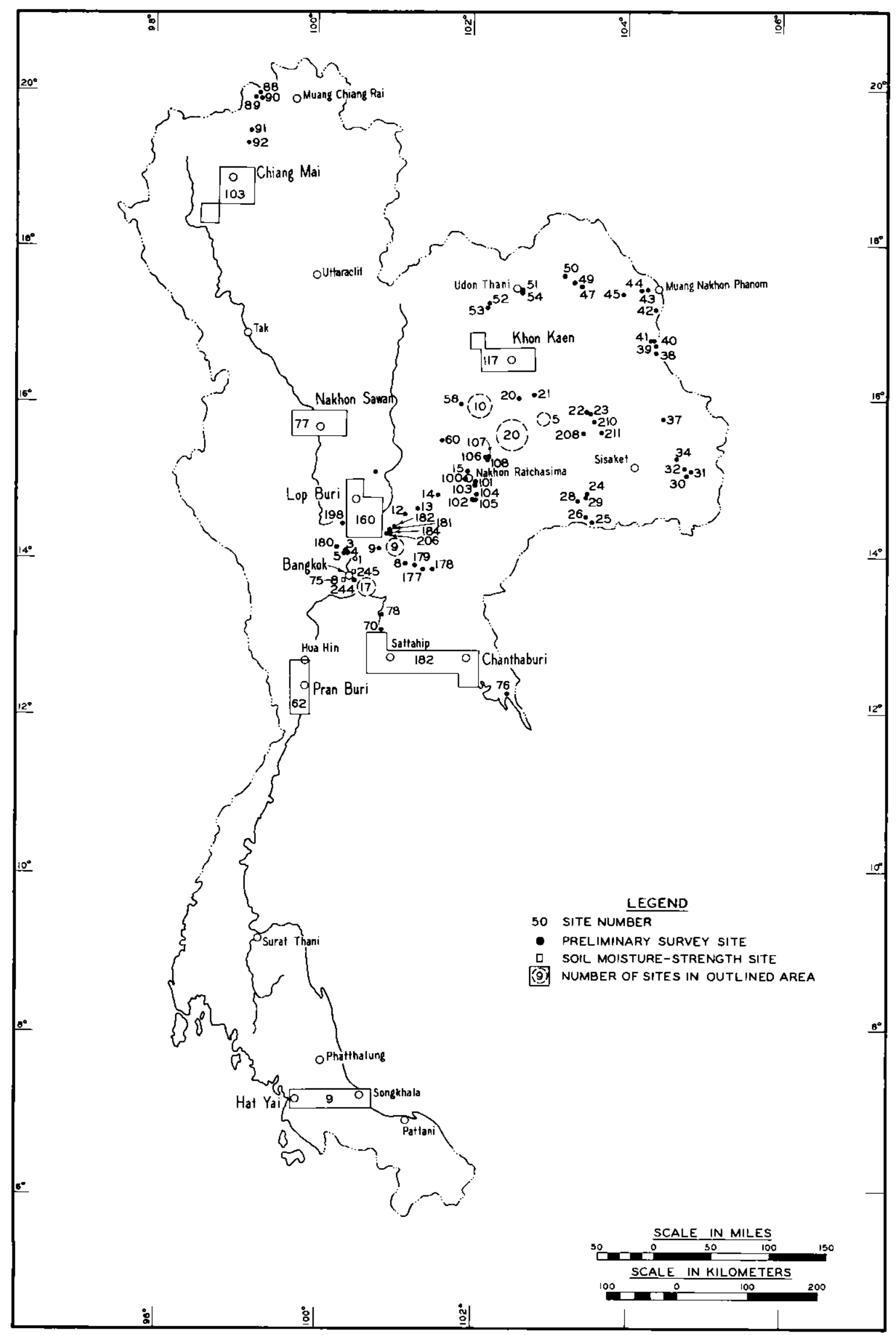

Fig. 4. General location of study sites 


\begin{tabular}{|c|c|c|c|c|c|c|c|}
\hline & $\begin{array}{c}\text { Prelim- } \\
\text { inary } \\
\text { survey }\end{array}$ & $\begin{array}{l}\text { Traffic- } \\
\text { ability } \\
\text { classi- } \\
\text { fication }\end{array}$ & $\begin{array}{l}\text { Sur- } \\
\text { face } \\
\text { Compo- } \\
\text { sition }\end{array}$ & $\begin{array}{l}\quad \text { Soil } \\
\text { Moisture- } \\
\text { Strength } \\
\end{array}$ & $\begin{array}{c}\text { CRREI } \\
\text { Air- } \\
\text { photo } \\
\text { Pattern } \\
\end{array}$ & $\begin{array}{l}\text { Terrain- } \\
\text { Vehicle } \\
\text { Tests } \\
\end{array}$ & Total \\
\hline Number of sites & 165 & 238 & 224 & 75 & 121 & 23 & 846 \\
\hline Mean and standard deviation & & & & & & & \\
\hline $\begin{array}{l}\text { High-moisture condition } \\
\text { CI, 0-6 in. } \\
\text { CI, 6-12 in. } \\
\text { RI and RCI, } 6-12 \text { in. } \\
\text { Sheargraph shear strength } \\
\text { Moisture content, } 0-6 \text { in. } \\
\text { Moisture content, } 6-12 \text { in. }\end{array}$ & $\begin{array}{l}70 \\
69 \\
55 \\
- \\
63 \\
61\end{array}$ & $\begin{array}{l}72 \\
72 \\
67 \\
43 \\
72 \\
72\end{array}$ & $\begin{array}{l}64 \\
63 \\
49 \\
30 \\
50 \\
50\end{array}$ & $\begin{array}{r}40 \\
40 \\
38 \\
4 \\
40 \\
40\end{array}$ & $\begin{array}{r}7 \\
7 \\
7 \\
-- \\
-- \\
--\end{array}$ & $\begin{array}{l}15 \\
15 \\
14 \\
15 \\
15 \\
14\end{array}$ & $\begin{array}{r}268 \\
266 \\
230 \\
92 \\
240 \\
237\end{array}$ \\
\hline
\end{tabular}

* Also used in analysis of cumulative frequency.

used in obtaining data in each program are discussed in Appendix $A$; the data are presented in tables Al-A6.

\section{Method of Computation}

30. The General Electric 225 electronic digital computer was employed in computations for this study. Two computer programs were required, one to compute the mean and standard deviation values and one to determine the frequency of occurrence of rating cone index. Data for these programs were supplied to the computer by means of punched IBM cards. These cards form a data retrieval system containing for each site the characteristics of the site (e.g. geographic location, topography class, topographic position, slope, land use, vegetation, etc.), soil data (e.g. percent grain sizes, Atterberg limits, USCS and USDA soil type, specific gravity, organic content, etc.), trafficability data (including CI, RI, RCI, sheargraph measurements, moisture content, and density for wet-season and high-moisture conditions, etc.), and climatological data (e.g. longtime average annual rainfall and temperature, etc.). For a given site, eight IBM cards are required to store approximately 125 pieces of information describing the site, soil, trafficability conditions, and cimate.

Mean and Standard Deviation Values of Soil Properties

31. This study establishes the statistical mean and standard deviation values of cone index and moisture content for the 0- to 6- and 
6- to 12-in. soil layers; remolding index, rating cone index, dry density, and specific gravity for the 6- to 12-in. soil layer; and sheargraph shear strength for the surface soil. Values for the dynamic soil properties, including moisture content, cone index, remolding index, rating cone index, and sheargraph shear strength, were computed for each of the three topography-moisture condition categories. Values for the static soil properties, including density and specific gravity, were computed only for a wet-season condition (high and low topography, respectively). Data are presented for each of the soil types in the USCS and USDA system in tables 1-7. The mean values in each table, except those for moisture content (table 5), are arranged from top to bottom in decreasing order; the moisture contents are arranged in increasing order. Where data are presented for both the 0- to 6- and 6- to 12-in. layers (tables 1 and 5), the soil types are arranged in order of mean values of the 6- to 12-in. layer. Where data are presented for soil-to-soil shear and soil-to-rubber shear (table 4), the soil types are arranged in order of mean values of soil-tosoil shear.

32. The data were analyzed in terms of mean $(\bar{x})$ and standard deviation ( $s$ ) because these are probably the most widely used and most readily understood statistical measures. The mean (commonly termed arithmetic mean or average) is computed by summing the individual measurements and dividing by the total number of measurements. The standard deviation is a measure of the dispersion of the data around the mean. The standard deviation for less than 30 measurements was computed by means of the formula

$$
s=\sqrt{\frac{\Sigma(x-\bar{x})^{2}}{n-1}}
$$

where

$$
\begin{aligned}
\Sigma= & \text { the sum of } \\
(\mathrm{x}-\overline{\mathrm{x}})= & \text { the deviation of an individual measurement from the mean } \\
& \text { of all measurements } \\
\mathrm{n}= & \text { the number of measurements }
\end{aligned}
$$

The -1 was omitted from the denominator of the formula when 30 or more 
measurements were used in the computation. When the number of measurements for the specific condition exceeds 30 , the interval defined by +1 and -1 standard deviation from the mean will usually contain approximately $68 \%$ of the data. Assuming the data are universally valid, if three additional measurements were taken, the values of two would usually fall within this interval. Mean and standard deviation values of a condition with fewer than 30 measurements, and especially of a condition with fewer than 5 measurements, should be viewed with skepticism.

33. The data in table 5 show that mean moisture contents for a given soil type are generally highest under a low-topography, high-moisture condition, intermediate under a low-topography, wet-season condition, and lowest under a high-topography, wet-season condition. If data had been developed for a high-topography, high-moisture condition, the mean moisture content would probably lie between those for the low-topography, wet-season condition, and the low-topography, high-moisture condition. This consistent pattern for all soil types (except a few with mean values that are based on relatively few observations and are therefore questionable) is presumed to be evidence in support of the proper identification of site data into the three arbitrary space-time categories used in this report. Analysis of strength

34. Cone index. The results of the analysis of CI are given in table 1. The data show mean cone indexes that are generally highest for the USCS coarse-grained soils with fines and USDA gravelly and sandy soils, intermediate for the USCS fine-grained soils and USDA loamy soils, and lowest for the USCS organic soil (OH) and USDA silty and clayey soils. Exceptions are the USCS low-plasticity soils (CI-ML and MU) of the 6- to 12-in. layer which have the highest mean cone indexes of all soils under low-topography, wet-season and high-moisture conditions. (The maximum mean value, for $\mathrm{GC}$, is based on one sample and, therefore, is not reliable.) The data also show higher cone indexes for the 6- to 12-in. soil layer than for the 0- to 6-in. layer; the mean cone indexes for all soils under each of the three topography-wetness conditions range from 57 to 78 units higher in the 6- to 12-in. layer than in the 0 - to 6-in. Iayer.

35. The mean and standard deviation values for each of the soil 
types and for all soils of a wetness condition are higher than those reported for temperate climates. ${ }^{I}$ This apparent difference can be accounted for by differences in the procedures used in the two studies for measuring cone index. In the temperate-soil study, the capacity of the cone penetrometer was 300 (0.5-in. cone), whereas in this study the capacity of the instrument was extended to $750(0.2-$ in. cone) in order to satisfy the needs for soil strength data for other engineering purposes. The average cone index for firm soil is higher when measured with the 0.2-in. cone. For example, the soil tested with the 0.5 -in. cone may give several 300+ readings, whereas the same soil tested with the 0.2-in. cone may give readings of 420,480 , etc.

36. Remolding index. The results of the analysis of RI are given in table 2. A comparison of the mean RI's for all soils shows lower average RI's for the low-topography positions. The data show an average RI of 1.03 for soils under high-topography, wet-season condition, an average RI of 0.76 for soils under low-topography, wet-season condition, and an average RI of 0.66 for soils under low-topography, high-moisture condition. Under each topography-moisture condition, the mean remolding indexes are generally highest for the USCS silty, coarse-grained and highly plastic, fine-grained soils and USDA sandy soils, lowest for the USCS lowplasticity, fine-grained soils and USDA loamy soils, and intermediate for the remaining soils. For the moisture levels considered in this report, relatively few soil types have mean RI's greater than 1.0.

37. Rating cone index. The results of the analysis of RCI are given in table 3. Like those for cone index, the data generally show rating cone indexes that are highest for the USCS coarse-grained soils with fines and USDA sandy soils, intermediate for the fine-grained soils, and lowest for the USCS organic soil (OH). The mean RCI for all soils under wet-season condition is about 60 units higher for high-topography than for lowtopography position (i.e. 180 versus $121 \mathrm{RCI}$ ), and the mean RCI for lowtopography position is 35 units higher under the wet-season condition than under the high-moisture condition (i.e. 121 versus 86 RCI). Because of test procedures that provide higher cone indexes in this study, the rating cone indexes are higher and the means and standard deviations are 
appreciably greater than those for comparable soils in a temperate climate (see paragraph 35 for an explanation).

38. Sheargraph shear strength. The results of the analysis of sheargraph shear strength of the surface soil for a normal load of 10 psi are given in table 4. The soil types are those for the 0 - to 6-in. layer. A comparison of the mean values for a given topography-moisture condition shows little difference between most soil types. The mean shear strengths for soil-to-soil and soil-to-rubber shear appear to be the highest for the high-plasticity and lowest for the low-plasticity USCS soils. A comparison of the mean values for all soils shows a decrease in strength with an increase in the moisture level of the soil for soil-to-soil shear. Only a slight decrease in strength is associated with an increase in moisture level. for soil-to-rubber shear. Because the number of samples for most soil types was insufficient for proper statistical analysis, and because the soil type of the 0-to 6-in. layer may, in some cases, be different from that of the surface soil, the results and conclusions drawn from this analysis should be viewed with caution. Analysis of moisture content

39. The results of the analysis of moisture content are given in table 5. The moisture content is inversely proportional to the soil strength. The data show mean moisture contents to be lowest for the USCS coarse-grained soils with fines and USDA gravelly and sandy soils, intermediate for the USCS low-plasticity, fine-grained soils and USDA loamy soils, and highest for the USCS high-plasticity and organic soils and USDA clayey soils. The mean moisture contents for all soils of the 0 - to 6 and 6- to 12-in. layers are about 5\% higher for low-topography than for high-topography, wet-season condition and those for low-topography position are about 4\% higher for high-moisture than for wet-season condition. Also, mean moisture contents of all soils are about $2 \%$ higher for the $0-$ to 6-in. layer than for the 6- to 12-in. layer.

Analysis of dry density

40. The results of the analysis of dry density are given in table 6 . Experience has indicated that changes in dry density of surface soils do not significantly affect their trafficability properties. However, the 
density data, along with specific gravity and moisture content data, can be used to estimate the percent saturation of a soil, which is an indicator of the degree of wetness. In this study the density data were analyzed for high- and low-topography, wet-season conditions. The data show densities that are generally highest for the USCS coarse-grained soils with fines and Iow-plasticity, fine-grained soils and USDA gravelly and sandy soils, intermediate for the USCS moderately plastic, fine-grained soils and USDA loamy soils, and lowest for the USCS highly plastic and organic soils and USDA clayey soils. The mean density for all soils is about 2 Ib per cu ft higher under a high-topography condition than under a low-topography, wetseason condition. A comparison of individual USCS soil types shows the density of all soils except CL and SM to be higher for the high-topography position than for the low-topography position. The CI and SM soil densities are slightly less in high positions than they are in low positions. Analysis of specific gravity

41. The results of the analyses of specific gravity are given in table 7. The specific gravity is a static soil property that does not vary with moisture content; consequently, the data were analyzed only for a wetseason condition. The mean specific gravity for all soils of high topography is 0.04 higher than that for all soils of low topography. Specific gravities are highest for the USCS plastic soils and USDA gravelly and clayey soils, and lowest for the USCS low-plasticity and organic soils and USDA silty and sandy soils.

\section{Cumulative Frequency Analysis of Rating Cone Index}

Procedures and presentation of data

42. The data used in this analysis are the same that were used in the mean and standard deviation analyses of RCI under wet-season and highmoisture conditions, respectively. The only difference between this and the previous analysis is in the statistical treatment of the data.

43. RCI's for each soil type under each topography-moisture condition were grouped into intervals of 10 RCI's from 1 to 300 and $300+$, i.e. 1 to 10,11 to 20,21 to $30 \ldots 291$ to 300 , and 300t. The measurements in 
each class for the group of $300+$ observations were tallied and their percentage of the total number was computed. The percentages were added cumulatively, starting with the percentage of $300+$ observations and progressing in order of decreasing RCI. Thus, the 300t or the larger value of the highest RCI increment for which data were available always was o\% frequency, and the smaller value for the lowest RCI increment for which data were available was $100 \%$ frequency. The RCI at $50 \%$ frequency is the median value.

44. The data are plotted in cumulative frequency graphs in plates 2-5 for the USCS soil types and in plates 6-9 for the USDA soil types. Graphs for three moisture conditions are usually shown for each soil type. Data were not available for sandy clay (USDA type) and OL and Pt (USCS types), nor were data available for analysis of one or more of the wetness conditions in some of the other soil types. The number of samples (sites) used in each analysis is indicated on its graph.

45. It is noted that where an appreciable number of samples were available for analysis, the three curves drawn for each soil type seldom cross each other. Also, the general range of RCI increases from the highmoisture graph through that for low-topography, wet-season, to the hightopography, wet-season graph. This is taken to be evidence of proper categorization of the basic field data into the three general moisture conditions.

46. Graphs are used to show the manner in which RCI varied. For example, the solid-line curve for CI soils in plate 4 shows that $10 \%$ of the CL soils under a low-topography, high-moisture condition had RCI's greater than 127, 20\% had values greater than 100, and 30\% had values greater than 90 , etc.

Estimating probability of vehicle "go"

47. The graphs can be used for estimating the probability of "go" for military vehicles. A soil for which the RCI is greatex than the VCI will permit 50 vehicles to pass in straight-line echelon or one vehicle to execute severe maneuvers. Thus, the cumulative frequency corresponding to the VCI indicates the probability of a vehicle's success in a given 
soil type under a given general moisture condition. For example, if it is known that the soil type is CL and that the water table is within 18 in. of the surface so that the soil is under a low-topography, high-moisture condition (but specific data on strength cannot be obtained), it can be hypothesized from plate 4 that the M48 tank (VCI $=49$ ) would have a $76 \%$ probability of "go."

Analysis for all soils

48. An analysis was made of the cumulative frequency of RCI for alI soils under wet-season and high-moisture conditions. The procedures of analysis were the same as those used in the analysis of each soil type (see paragraph 43). The curves developed from the analysis may be used to estimate the percentage of areas trafficable for a given vehicle under a given condition of moisture. A discussion of the curves and their use is presented in paragraph 73 . 
PART TV: SOIL TRAFFICABILITY CLASSIFICATION

SCHEME AND RELATED STUDIES

49. The soil trafficability classification scheme shown in tables 8 and 9 is essentially a listing of soil types in descending order of their median rating cone indexes under three conditions of moisture: high and low topography under wet-season conditions, and low topography under a highmoisture condition. Information for a high-topography, high-moisture condition was not included in the scheme because data were too few to permit proper analysis. Soil types according to both the USCS and the USDA soil classification system are employed. Thus the scheme can be considered a sixfold scheme for the classification of soils from a trafficability standpoint. The scheme considers the strength of soils in the 6- to 12-in. Iayer.

50. This part of the report summarizes the vehicle classification categories developed in an earlier study, and describes the soil trafficability classification scheme and its possible application in detail. Tables 10 and 11 supplement the classification scheme by providing specific data on the percent probability of "go" for military vehicles on level and sloping terrain for each of the three general moisture conditions and the two soil classification systems.

\section{Vehicle Categories}

51. Different military vehicles require different minimum soil strengths for operation. A soil condition that is easily trafficable for one vehicle may be impassable for another. Therefore, in order to make the soil trafficability classification meaningful, it was necessary to incorporate vehicle requirements into the scheme for estimating the probability of vehicle "go."

52. In an earlier study 15 a system was developed for classifying vehicles on the basis of the minimum soil strength each required for 50 straight-line passes or one severe maneuver on level ground. This system is condensed and repeated here. 


\begin{tabular}{|c|c|c|}
\hline $\begin{array}{l}\text { Cate- } \\
\text { gory }\end{array}$ & $\begin{array}{c}\text { VCI } \\
\text { Range }\end{array}$ & Vehicle and Vehicle Types \\
\hline 1 & $20-29$ & $\begin{array}{l}\text { M29C weasel, M76 otter., Canadian snowmobile, and some } \\
\text { lightweight experimental. vehicles. Example: VCI of } \\
\text { M29C weasel }=25\end{array}$ \\
\hline 2 & $30-49$ & $\begin{array}{l}\text { Engineer and high-speed tractors with comparatively wide } \\
\text { tracks and low contact pressures. Examples: VCI of D7 } \\
\text { engineer tractor }=40 \text {; VCI of } \mathrm{M} 14 \text { armored personnel } \\
\text { carrier }=37\end{array}$ \\
\hline 3 & $50-59$ & $\begin{array}{l}\text { Tractors with average contact pressures, tanks with com- } \\
\text { paratively low contact pressures, and some trailed ve- } \\
\text { hicles with very low contact pressures. Example: VCI } \\
\text { of M48 medium tank }=52\end{array}$ \\
\hline 4 & $60-69$ & $\begin{array}{l}\text { Most medium tanks, tractors with high contact pressures, } \\
\text { and all-wheel-drive trucks and trailed vehicles with } \\
\text { low contact pressures. Example: VCI of Ml35, 2-1/2- } \\
\text { ton truck }=62 \text {. }\end{array}$ \\
\hline 5 & $70-79$ & $\begin{array}{l}\text { Most all-wheel-drive trucks, a great number of trailed } \\
\text { vehicles, and heavy tanks. Example: VCI of } 1-1 / 2 \text {-ton, } \\
4 \times 4 \text { dump truck }=73\end{array}$ \\
\hline 6 & $80-99$ & $\begin{array}{l}\text { A great number of all-wheel-drive and rear-wheel-drive } \\
\text { trucks, and trailed vehicles intended primarily for } \\
\text { highway use. Example: VCI of } 1 / 2 \text {-ton, } 4 \times 2 \text { pickup } \\
\text { truck }=88\end{array}$ \\
\hline 7 & $\begin{array}{l}100 \\
\text { or } \\
\text { greater }\end{array}$ & $\begin{array}{l}\text { Rear-wheel-drive vehicles and others that generally are } \\
\text { not expected to operate off roads, especially in wet } \\
\text { soils. Example: VCI of } 5 \text {-ton, } 4 \times 2 \text { dump truck }=119\end{array}$ \\
\hline
\end{tabular}

The vehicle cone indexes for individual vehicles within the categories are included in Appendix A of reference 15.

\section{Soil Trafficability Classification Scheme, Level Terrain}

53. The soil trafficability classification scheme for level terrain is presented in USCS terms in table 8 for high and low topography under wetseason conditions, and for low topography under a high-moisture condition. The scheme is presented in USDA terms in table 9 for the same set of moisture conditions. Information presented in the scheme for each soil type includes a general estimate of the probability of "go" on level terrain for vehicles of various categories. Measurements of soil strength are also included. 
54. For the sake of simplicity of presentation, the percent probabilities of vehicle "go" have been arbitrarily classified as follows:

$\begin{array}{ll}\text { Excellent } & \text { greatex than } 90 \% \text { probability of "go" } \\ \text { Good } & 76 \text { to } 90 \% \text { probability of "go" } \\ \text { Fair } & 50 \text { to } 75 \% \text { probability of "go" } \\ \text { Poor } & 10 \text { to } 49 \% \text { probability of "go" } \\ \text { No "go" } & \text { less than } 10 \% \text { probability of "go" }\end{array}$

The probability-of-"go" information is illustrated in tables 8 and 9 by a series of bar graphs, one for each soil type.

Procedures for deriving "go" information

55. The vehicle cone indexes corresponding to 10, 50, 75, and $90 \%$ probability of "go," the limiting values of the vehicle "go" groupings, were derived from the cumulative frequency rating cone index graphs (plates 2-9). For example, from the CI soil graph for a low-topography, highmoisture condition (plate 4) it can be seen that the RCI's at 10, 50, 75, and $90 \%$ cumulative frequency are 128, 74, 50, and 39, respectively. This means that the soil strength will be greater than 128 RCI 10 times out of 100, greater than 74 RCI 50 times out of 100, greater than 50 RCI 75 times out of 100, and greater than 39 RCI 90 times out of 100. Table 8 shows that vehicles with a VCI greater than 128 will have less than a $10 \%$ probability of "go"; those with a VCI ranging from 74 to 128 will have $50 \%$ probability of "go"; those with a VCI ranging from 50 to 74 will have a 50 to $75 \%$ probability of "go"; those with a VCI ranging from 39 to 50 will have a 76 to 90\% probability of "go"; and those with a VCI less than 39 will have greater than $90 \%$ probability of "go."

Reliability of "go" information

56. The probability lines delineating the vehicle "go" groupings on the bar graphs in tables 8 and 9 are solid where the data were based on more than four samples and the information shown was considered to be reliable. The lines are broken where less than five samples were used in the analysis or the data were otherwise questionable. The positioning of these 
broken lines was based on an assumed RCI estimated from the textural, plasticity, and organic properties of the soil.

57. It should be noted particularly that the occurrence of obstacles was not considered in the probability of "go" estimates for level or sloping terrain. Obstacle components of terrain, such as trees, hedges, dikes, and streams, that present a definite deterrent or obstruction to mobility of vehicles would certainly decrease the probability of "go."

Soil strength information

58. The mean CI, RI, surface sheargraph shear strength for a load of $10 \mathrm{psi}$, and RCI, and the range of RCI (discussed in Part III) are presented again in tables 8 and 9. It may be noted that the mean RCI for a soil generally is greater than its median RCI, which is the same value as the VCI at $50 \%$ probability of "go."

\section{Probability of Vehicle "Go" on Level and Sloping Terrain}

59. The percent probabilities of vehicle "go" on level and sloping soils classified in terms of the USCS are presented in table 10 for both high and low topography under wet-season conditions, and for low topography under a high-moisture condition; these data on soils classified in terms of the USDA system are presented in table 11. The data for each soil typemoisture condition include the probabilities of negotiation of level terrain (O\% slope) and slopes of 15, 30, and 45\% by vehicles in each of the seven vehicle categories. The probabilities were established for the median VCI within vehicle categories 1 through 6 (i.e. 25 VCI for category 1, 40 VCI for category 2, etc.) and for the minimum VCI (100) in category 7, for tracked vehicles with grousers shorter than $1-1 / 2$ in. and for wheeled vehicles. Tracked vehicles with grousers longer than $1-1 / 2$ in. would have a slightly better probability of "go" on sloping soils than that computed for tracked vehiclès with shorter grousers. For all practical purposes, however, the difference is insignificant, and the probabilities of "go" listed under the "tracked" column in tables 10 and 11 may be applied to both types of tracked vehicles. The probability of "go" established for a vehicle with a median VCI of a category will closely approximate and may 
be used to estimate the probabilities of "go" for other vehicles within the same category.

Procedure for deriving "go" information

60. The probability data were obtained from the cumulative frequency rating cone index graphs presented in plates 2-9. If VCI is substituted for RCI and probability of "go" for cumulative frequency, an estimate of the probability of "go" on level terrain can be made for any vehicle for which a VCI has been computed (discussed in paragraph 47). In order to determine the probability of "go" for a given slope, the slope index, derived from the curve of the vehicle type shown in plate 1 , was added to the VCI and the probability of "go" for the soil type-moisture condition was based upon the cumulative frequency reading for this new VCI value. For example, the probabilities of "go" for tracked and wheeled vehicles of 55 VCI (median VCI of category 3) on $0,15,30$, and 45\% slopes of a silt loam soil area under low-topography, wet-season condition were derived as follows. The VCI was substituted for RCI in the abscissa, and the probability of "go" was substituted for cumulative frequency in the ordinate of the silt loam low-topography, wet-season condition graph shown in plate 8 . At 55 VCI the probability of "go," read from the graph, was $87 \%$. This value applies to tracked and wheeled vehicles at $0 \%$ slope. The slope index at $15 \%$ slope, read from the curves of plate 1, was 7 for tracked vehicles with grousers shorter than $1-1 / 2$ in. and 9 for wheeled vehicles. This index was added to the VCI to provide values of 62 (55 plus 7) for the tracked vehicles and 64 (55 plus 9) for the wheeled vehicles. The probabilities of "go" for the VCI values of 62 and 64, read from the graph in plate 8 for silt loam, low topography, and the wet season, were 80 and $79 \%$, respectively. At $30 \%$ slope, the slope indexes were 15 and 20, the VCI's became 70 and 75 , and the resulting probabilities of "go" were 74 and $68 \%$ for the two vehicle types, respectively; at 45\% slope, the slope indexes were 27 and 40, the VCI's became 82 and 95, and the probabilities of "go" read from the graph were 60 and 49\%, respectively. The probability of "go" can be estimated for any slope and for any vehicle for which a VCI has been computed by using data read from the proper soil type-moisture condition graph and 
slope index curve, and following the procedures discussed above. Reliability of "go" information

61. The probability values for a wet-season condition are undoubtedly influenced by the high-moisture, low-strength bias associated with the basic data (previously discussed in paragraph 24aㅡ); thus, the actual probability of "go" would be somewhat higher than that indicated.

62. The number of samples used in the analysis of a particular soil type-moisture condition provides a rough estimate of its reliability. Analyses based on more than 30 samples would generally have a small plus and minus probability error, i.e. the true probability based on an infinite number of the same type of samples would not vary by more than plus or minus a small standard error of estimate. The probabilities of "go," therefore, are considered to be of good reliability. An analysis based on fewer than 30 samples and especially fewer than 15 samples, but more than 4 samples, would have a moderate standard error of estimate (estimated at \pm 10 to $\pm 25 \%$ probability of "go"). Probabilities based on an analysis of this number of samples are considered to be of only fair reliability and should be viewed with skepticism. Five was arbitrarily chosen as the minimum number of samples needed to provide a reasonably reliable probability value; probabilities of "go" were only estimated for the analyses based on fewer than 5 samples. The estimations were based on assumed strengths estimated from the textural, plasticity, and organic properties of the soil.

\section{Application of Information for Estimating Trafficability Conditions}

63. The information presented in the trafficability classification scheme and probability of "go" tables should be especially useful in military intelligence, military-operations planning, and vehicle-design work. The information may be applied in quantitative or qualitative terms to military problems or to studies of a tactical or strategic nature.

64. The information can be used to estimate trafficability conditions for areas in Southeast Asia that, in most cases, will not be accessible for measurement. Information needed for proper analysis includes 
climate and weather, topographic position or water-table conditions, and soil type. Climatological and weather data can be obtained from meteorological records; data on topographic position (and slope if desired) are available from large-scale topographic maps, and information on soil type can be obtained from engineering or pedological reports. It may be reasoned that trafficability-prediction information would not be needed for accessible areas because direct strength measurements with the cone penetrometer could be taken where and when desired. The information, however, could be used in these areas to facilitate a particular study, e.g. the speedy selection of one of several possible access routes, the selection of possible barrier areas (mine fields, etc.) that normally would have good to excellent probabilities of "go," or the selection of broad areas providing the best positions for offensive or defensive operations.

Use of trafficability classification scheme

65. The following paragraphs explain by means of examples how the classification scheme can be used.

66. Season, soil type, and topography. If it is known that the season is the wet season, the soil type is CI, and the topography is low topography, the data in table 8 for low-topography, wet-season condition would be used to determine trafficability. In this case, the probability of "go" on the CI soil would be less than $10 \%$ for vehicles with VCI's greater than 185 , between 10 and $50 \%$ for vehicles with VCI's between 89 and 185, between 50 and $75 \%$ for vehicles with VCI's between 58 and 89 , between 76 and $90 \%$ for vehicles with VCI's between 42 and 58 , and greater than $90 \%$ for vehicles with VCI's less than 42.

67. Season, soil type, topography, plus rainy weather or high-watertable condition. If, in addition to the knowledge of the season, soil type, and topography, it is known that the soil has been subjected to several days of rain, or if a high water table is known to exist, the lowtopography, high-moisture condition data presented in table 8 (or table 9 for USDA soils) would be used. The probability of venicle "go" on CL soils under these conditions would be less than $10 \%$ for vehicles with VCI's greater than 128, between 10 and 50\% for vehicles with VCI's between 74 and 
128, between 50 and $75 \%$ for vehicles with VCI's between 50 and 74 , between 76 and $90 \%$ for vehicles with VCI's between 39 and 50, and greater than 90\% for vehicles with VCI's less than 39.

68. Probability of one straight-line pass for a vehicle. For clayey soils, an RCI equal to about $50 \%$ of the VCI usually will permit one straight-line pass of the vehicle. ${ }^{13}$ The probability of a successful operation may be derived from the classification scheme (tables 8 and 9 ) by projecting a line down from the VCI value multiplied by one-half and reading the probability at its intersection with the particular graph of soil type-wetness condition under consideration. For example, a vehicle with a VCI of $100^{\circ}$ would have a recomputed index of 50 ( $\left.100 \times 0.50\right)$. The probability of its making one straight-line pass on a CL soil under lowtopography, wet-season condition (from table 8) would be 76 to $90 \%$ (estimated at $83 \%$ ).

Use of probability of "go" tables

69. The following paragraphs explain how the probability tables (tables 10 and II) can be used. The particular data to be used, like that for the soil trafficability classification scheme, will depend upon the amount and type of information known, i.e. the topography, moisture condition, and the soil type and system in which the soil is classified.

70. Probability of "go" for vehicles within specific VCI categories. The probability of "go" on sloping ground may be estimated for tracked or wheeled vehicles within VCI categories. If, for example, a low-topography, high-moisture condition prevails and the soil is a CI with a $15 \%$ slope, the probability of "go" for tracked vehicles in category 3 (VCI 50 to 59) would be $63 \%$ (from table 10 ).

71. Comparison of probabilities between two vehicle categories. The probabilities of "go" can be compared for vehicles in two different categories to estimate the advantage that vehicles in one category would have over vehicles in another. For example, under the same set of conditions as those stated in the preceding paragraph, tracked vehicles in category 5 (VCI 70 to 79) would have a $40 \%$ probability of "go" (table 10). Since the table shows the probability of "go" for vehicles in category 3 to be $63 \%$, the difference, 23\% ( 63 minus 40), indicates the advantage in performance of vehicles in category 3 . 
72. Comparison of probabilities for different soil types and slopes. The probabilities of "go" for vehicles within a given category can be compared for two or more different soil types and slopes in order to determine quantitatively the advantage that one route would have over another. For example, if tracked vehicles in category 3 were being considered for use in a low-topography area under a high-moisture condition (table 10), the probability of "go" along route $\mathrm{A}$, a $\mathrm{CH}$ soil with maximum slopes of $30 \%$, would be 40\%; the probability of "go" along route B, a CL soil with maximum slopes of $15 \%$, would be $63 \%$. Thus, from the standpoint of soil type and slope, route $B$ would have a decided advantage of $23 \%$ (63 minus 40 ) over route $A$.

\section{Estimation of Percentage of Area Trafficable}

73. Cumulative frequency curves of the RCI data for all fine-grained soils and coarse-grained soils with fines tested in Thailand are shown for wet-season (high and low topography) and high-moisture (low topography) conditions in plate 10. For purposes of comparison, a similar set of curves is also shown for humid-temperate soils of the United States. The cumulative frequency of RCI, in percent, is plotted for a 10-300 range of RCI. The curves in plate 10 permit one to estimate the percentage of area trafficable for a given vehicle under a wet-season or high-moisture condition. Because the data are biased toward wetter-than-average conditions, estimates of percentages of trafficable areas made from the curves will be smaller than actual, i.e. on the conservative side. Examination of the data reveals that in Thailand a vehicle with a VCI of 80 can make 50 passes in $60 \%$ of the soil areas under average conditions in the wet season, and in $40 \%$ of the low-lying soil areas under poorest trafficability conditions (high-moisture condition). The same vehicle can make 1 pass (vehicle cone index is $80 \times 0.50$ or 40 for 1 pass) in $89 \%$ of the area under average conditions in the wet season and in 79\% of the low-lying areas under poorest trafficability conditions. It should be emphasized that passable areas are considered strictly in terms of the bearing strength of soils on level surfaces. The presence and orientation of slopes and obstacies and 
consideration of the extent of areas of sand would affect the percentage of area trafficable. A comparison of the curves for Thailand and U. S. soils shows that the Thailand soils have slightly higher strengths (68 median RCI for the Thailand soils versus 63 for the U. S. soils) under high-moisture conditions and slightly lower strengths (97 median RCI for the Thailand soils versus 107 for the U. S. soils) under wet-season conditions. 
Conclusions

74. Based on the data and discussions presented herein, it is concluded that the scheme for classifying trafficability of Thailand soils has the following advantages:

a. It rates soil types (both USCS and USDA) according to their median rating cone index under high- and low-topography, wet-season conditions, and under low-topography, highmoisture condition.

b. From a consideration of cumulative frequency of occurrence of rating cone index, it permits a ready estimate of the chances of successful travel of any military vehicle (whose vehicle cone index is known) on any soil type under three space-time moisture conditions.

75. The conclusions that follow are based on the soil information derived from the various analyses of the basic data.

a. Soil strength.

(1) Soils in low-lying positions (low topography) generally have lower strengths than those in high-lying positions (high topography). (Paragraphs 34-38 and tables 1-4.)

(2) The initial strength (cone index) of the 6- to 12-in. soil layer ranges from 57 to 78 units higher than that of the 0- to 6-in. layer. (Paragraph 34 and table 1.)

(3) The remolding indexes are generally highest for the silty coarse-grained soils and highly plastic finegrained soils and lowest for the low-plasticity and loamy fine-grained soils. (Paragraph 36 and table 2.)

(4) The remolding index of a soil decreases with an increase in the moisture level. Under highest moisture level (poorest trafficability condition) the soils retain an average of two-thirds of their initial strength after remolding. (Paragraph 36 and table 2.)

(5) The initial and remolded strengths of soils (cone index and rating cone index) are highest for the USCS coarsegrained soils with fines and USDA sandy soils, intermediate for the fine-grained soils, and lowest for the organic soils. The rating cone index averages 60 units higher for high-topography than for low-topography position, and that for low-topography positions averages 35 units higher under wet-season condition than under 
high-moisture condition. (Paragraphs 34 and 37 and tables 1 and 3.)

(6) Soils in Thailand have slightly higher strengths under high-moisture condition and slightly lower strengths under wet-season condition than do soils in humidtemperate areas of the United States. (Paragraph 73 and plate 10.)

b. Soil-moisture content.

(1) For a given topography-moisture level the moisture contents are lowest for the USCS coarse-grained soils with fines and USDA sandy soils, intermediate for the USCS low-plasticity, fine-grained soils and USDA loamy soils, and highest for the USCS high-plasticity and organic soils and USDA clayey soils. (Paragraph 39 and table 5.)

(2) The average moisture contents in the wet season are about 5\% higher for low-topography than for hightopography positions, and those in low-topography positions are about $4 \%$ higher under high-moisture than under wet-season conditions. (Paragraph 39 and table 5.)

c. Density.

(1) The densities are generally highest for the USCS coarsegrained soils with fines and low-plasticity fine-grained soils and USDA gravelly and sandy soils, and lowest for the USCS highly plastic and organic soils and USDA clayey soils. (Paragraph 40 and table 6.)

(2) The average density is about 2 Ib per cu ft higher under high-topography than under low-topography, wet-season condition. (Paragraph 40 and table 6.)

d. Specific gravity.

(1) Specific gravities are highest for the USCS plastic soils and USDA gravelly and clayey soils, and lowest for the USCS low-plasticity and organic soils and USDA silty and sandy soils. (Paragraph 41 and table 7.)

(2) The specific gravity of soils on high-topography position averages 0.04 more than that on low-topography position.

e. Probability of "go."

(1) The probability of "go" for a given vehicle on a given soil type is highest for high-topography, wet-season condition, intermediate for low-topography, wet-season condition, and lowest for low-topography, high-moisture condition. For a given topography-moisture condition, the probability of "go" decreases for soils in the 
following order: clean, coarse-grained soils, coarsegrained soils, coarse-grained soils with fines, finegrained soils, and organic soils. (Table 8.)

(2) On a basis of soil strength only, vehicles with vehicle cone indexes less than 80 (i.e. practically all military vehicles except those intended primarily for highway use) can negotiate at least $60 \%$ of the soil areas during average wet-season conditions and at least $40 \%$ of the areas during poorest trafficability conditions. (Paragraph 73 and plate 10.)

\section{Recommendations}

76. It is recommended that:

a. In order to improve the reliability of the probability-of"go" information that has been derived from a statistical analysis of existing data, new or additional rating cone index information should be collected on USCS soil typemoisture conditions with fewer than 15 observations. This information should include data from all soil types except SM and CL under high-topography, wet-season condition; SP-SM, SM-SC, and organic soil types under low-topography, wet-season condition; and SP-SM, SC, CL-ML, and organic soil types under low-topography, high-moisture condition.

b. Sheargraph measurements should be incorporated in the trafficability classification scheme if investigations in progress show that these measurements can be related to vehicle traction. 
1. U. S. Army Engineer. Waterways Experiment Station, CE, Trafficability of Soils; Soil Classification, by M. P. Meyer and S. J. Knight. Technical Memorandum No. 3-240, Supplement 16, Vicksburg, Miss., August 1961.

2. , Environmental Factors Affecting Ground Mobility in Thailand; Appendix C: ' Soil Trafficability. Technical Report No. 5-625, Vicksburg, Miss., May 1963.

3. , A Quantitative Method for Describing Terrain for Ground Mobility; Surface Composition. Technical Memorandum No. 3-726, vol II, Vicksburg, Miss. (not yet published).

4. Soil Moisture-Strength Characteristics of Selected Soils in Thailand (not yet published).

5. Mobility Environmental Research Study: A Quantitative Method for Describing Terrain for Ground Mobility; Selected Air-Photo Patterns of Terrain Features, by R. E. Frost and others. Technical Report No. 3-726, vol VI, Vicksburg, Miss., May 1966.

6. An Analytical Model for Predicting Cross-Country Vehicle Performance (not yet published).

7. , The Unified Soil Classification System. Technical Memorandum No. 3-357, vol I, Vicksburg, Miss., March 1953 (Rev April 1960).

8. U. S. Bureau of Plant, Industry, Soils, and Agriculture Engineering, Soil Survey Manual. U. S. Department of Agriculture Handbook No. 18, U. S. Government Printing Office, Washington, D. C., August 1951.

9. U. S. Army Engineer Waterways Experiment Station, CE, Trafficability of Soils; A Sumary of Trafficability Studies Through 1955, by S. J. Knight. Technical Memorandum No. 3-240, Supplement 14, Vicksburg, Miss., December 1956.

10. U. S. Department of the Army, Soils Trafficability. Technical Bulletin TB ENG 37, U. S. Government Printing Office, Washington, D. C., July 1959.

11. Cohron, Gerald T., "Soil sheargraph." Agricultural Engineering, vol 44, No. 10 (October 1963), pp 554-556.

12. "Cohron sheargraph for shearing strength measurements." Journal, Environmental Sciences, vol 6, No. 6 (December 1963), pp 17-20.

13. U. S. Army Engineer Waterways Experiment Station, CE, One-Pass Performance of Vehicles on Fine-Grained Soils, by C. J. Nuttall, Jr., C. W. Wilson, and R. A. Werner. Contract Report No. 3-152, Vicksburg, Miss., July 1966. 
14. U. S. Army Engineer Waterways Experiment Station, CE, Water Table Prediction Study (not yet published).

15. Trafficability of Soils; Vehicle Classification. Technical Memorandum No. 3-240, Supplement 9, Vicksburg, Miss., May 1951. 
Table 1

Mean and Standard Deviation Values for USCS and USDA Soil Types

Cone Index, 0- to 6-in. and 6- to 12-in. Layers

\begin{tabular}{|c|c|c|c|c|c|c|c|c|c|c|c|c|c|}
\hline \multicolumn{7}{|c|}{ USCS } & \multicolumn{7}{|c|}{ USDA } \\
\hline \multirow[b]{2}{*}{ Type } & 0 & \multicolumn{2}{|c|}{$\begin{array}{l}\text { to } 6 \text {-in. } \\
\text { Layer }\end{array}$} & \multicolumn{3}{|c|}{$\begin{array}{l}\text { 6- to 12-in. } \\
\text { Layer }\end{array}$} & \multirow[b]{2}{*}{ Type } & \multicolumn{3}{|c|}{$\begin{array}{l}0-\text { to } 6-i n . \\
\text { Layer }\end{array}$} & \multicolumn{3}{|c|}{$\begin{array}{l}\text { 6- to } 12-\text { in. } \\
\text { Layer }\end{array}$} \\
\hline & $\mathrm{n}$ & $\overrightarrow{\mathrm{x}}$ & $\bar{s}$ & $\mathrm{n}$ & $\overline{\bar{x}}$ & $\bar{s}$ & & $\mathrm{n}$ & $\overline{\bar{x}}$ & $s$ & $n$ & $\overline{\mathrm{x}}$ & $\mathrm{s}$ \\
\hline & & & & & Topog & aphy & Season & & & & & & \\
\hline $\mathrm{GC}$ & -- & -- & -- & 1 & 617 & -- & GL & 2 & 137 & 76 & 4 & 617 & 188 \\
\hline GM & -. & -- & -- & 2 & 462 & 407 & GSCL & 1 & 171 & -- & 2 & 524 & 320 \\
\hline SP-SM & 1 & 273 & -- & -- & -- & -- & $\mathrm{SC}$ & -- & -- & -- & 2 & 511 & 338 \\
\hline SM-SC & 3 & 163 & 71 & 10 & 378 & 238 & GSI & 1 & 273 & -- & 7 & 340 & 258 \\
\hline ML & 13 & 197 & 115 & 11 & 304 & 259 & LS & 17 & 290 & 164 & 22 & 301 & 226 \\
\hline $\mathrm{SC}$ & 7 & 155 & 85 & 17 & 286 & 187 & CL & 9 & 204 & 162 & 8 & 278 & 207 \\
\hline SM & 42 & 243 & 149 & 59 & 269 & 209 & SI & 35 & 184 & 129 & 49 & 264 & 189 \\
\hline $\mathrm{CL}$ & 8 & 100 & 63 & 23 & 258 & 221 & SiC & 2 & 127 & 83 & 2 & 252 & 27 \\
\hline $\mathrm{CH}$ & 8 & 168 & 185 & 15 & 205 & 163 & $\mathrm{~L}$ & 19 & 149 & 106 & 28 & 218 & 206 \\
\hline MH & 17 & 128 & 66 & 14 & 179 & 76 & SiI & 10 & 98 & 42 & 6 & 210 & 261 \\
\hline CL-ML & 3 & 89 & 18 & 8 & 135 & 41 & SCL & 6 & 145 & 63 & 9 & 199 & 133 \\
\hline & & & & & & & GCL & -- & -- & -- & 2 & 198 & 33 \\
\hline & & & & & & & SiCL & 1 & 126 & -- & 4 & 188 & 121 \\
\hline & & & & & & & $\mathrm{C}$ & 3 & 142 & 79 & 6 & 171 & 51 \\
\hline & & & & & & & $\mathrm{S}$ & 5 & 215 & 72 & 6 & 153 & 37 \\
\hline & & & & & & & GSiL & -- & -- & -- & 1 & 125 & - \\
\hline soil & 102 & 188 & 132 & 160 & 258 & 200 & AII soj & בגבו & 185 & 129 & 158 & 263 & 205 \\
\hline
\end{tabular}

$\begin{array}{llllllllllllll}\text { All soils } & 102 & 188 & 132 & 160 & 258 & 200 & \text { All soils } & 111 & 185 & 129 & 158 & 263 & 205\end{array}$

Low-Topography, Wet-Season Condition

\begin{tabular}{|c|c|c|c|c|c|c|c|c|c|c|c|c|c|}
\hline $\mathrm{GC}$ & -- & -- & -- & 1 & 430 & - & GCL & 1 & 98 & -- & 4 & 522 & 293 \\
\hline CL-ML & 12 & 156 & 103 & 41 & 313 & 188 & GL & -- & -- & -- & 2 & 385 & 198 \\
\hline$M L$ & 51 & 192 & 151 & 50 & 303 & 206 & GSiCL & -- & -- & -- & 1 & 371 & - \\
\hline $\mathrm{SM}$ & 61 & 239 & 160 & 95 & 282 & 176 & GSI & 3 & 246 & 150 & 5 & 349 & 216 \\
\hline $\mathrm{SC}$ & 10 & 181 & 145 & 35 & 264 & 190 & GSCL & - & -- & - & 2 & 290 & 198 \\
\hline SM-SC & 7 & 248 & 134 & 20 & 250 & 183 & SL & 84 & 218 & 155 & 140 & 252 & 171 \\
\hline CL & 84 & 155 & 152 & 241 & 209 & 260 & IS & 28 & 186 & 1.16 & 40 & 250 & 4 \\
\hline$S P-S M$ & 1 & 100 & - & 9 & 193 & 103 & $S$ & 5 & 233 & 289 & 14 & 240 & 46 \\
\hline $\mathrm{CH}$ & 57 & 74 & 49 & 119 & 134 & 90 & $\mathrm{CL}$ & 14 & 100 & 55 & 51 & 229 & 59 \\
\hline $\mathrm{MH}$ & 28 & 99 & $4 \sqrt{4}$ & 35 & 125 & 73 & $I$ & 57 & 145 & 125 & 122 & 221 & 72 \\
\hline $\mathrm{OH}$ & 8 & 52 & 45 & 6 & 31 & 13 & SiL & 83 & 142 & 123 & 98 & 210 & \\
\hline & & & & & & & SCL & 3 & 104 & 45 & 34 & 195 & \\
\hline & & & & & & & $\mathrm{C}$ & 25 & 63 & 35 & 43 & 184 & \\
\hline & & & & & & & SiCL & 32 & 1.14 & 129 & 51 & 154 & \\
\hline & & & & & & & Si & 4 & 300 & 299 & 2 & 141 & \\
\hline & & & & & & & SiC & 23 & 63 & 58 & 39 & 97 & \\
\hline soils & 319 & 158 & 142 & 652 & 217 & 168 & All soils & 362 & 152 & 138 & $6+8$ & 216 & \\
\hline
\end{tabular}

Low-Topography, High-Moisture Condition

\begin{tabular}{|c|c|c|c|c|c|c|c|c|c|c|c|c|c|}
\hline CL-ML & 7 & 119 & 117 & 20 & 266 & 209 & GSI & -- & -- & -- & 1 & 311 & -- \\
\hline ML & 28 & 124 & 109 & 21 & 257 & 221 & $S i$ & 2 & 337 & 392 & -- & -- & . \\
\hline SM-SC & 1 & 249 & -- & 4 & 249 & 259 & LSS & 9 & 145 & 102 & 13 & 208 & 144 \\
\hline $\mathrm{SM}$ & 19 & 171 & 159 & 32 & 201 & 114 & $\mathrm{~S}$ & 4 & 259 & 327 & 6 & 207 & 134 \\
\hline $\mathrm{SC}$ & 2 & 68 & 8 & 12 & 167 & 82 & GCL & 1 & 103 & - & - & $\cdots$ & -- \\
\hline CL & 38 & 74 & 30 & 98 & 127 & 55 & SiL & 23 & 85 & 40 & 26 & 190 & 180 \\
\hline MH & 13 & 91 & 34 & 16 & 116 & 66 & SL & 30 & 126 & 75 & 46 & 178 & 141 \\
\hline SP-SM & 1 & 78 & -- & 2 & 100 & 35 & I & 31 & 83 & 36 & 58 & 167 & 139 \\
\hline $\mathrm{CH}$ & 29 & 47 & 24 & 53 & 94 & 57 & CL & 12 & 76 & 39 & 22 & 158 & 76 \\
\hline \multirow[t]{4}{*}{$\mathrm{OH}$} & 4 & 30 & 17 & 4 & 35 & 14 & SCL & - & -- & -- & 15 & 123 & 54 \\
\hline & & & & & & & C & 16 & 61 & 35 & 18 & 122 & 60 \\
\hline & & & & & & & SiCL & 15 & 63 & 37 & 30 & 105 & 58 \\
\hline & & & & & & & $\mathrm{SiC}$ & 20 & 48 & 27 & 27 & 76 & 43 \\
\hline
\end{tabular}

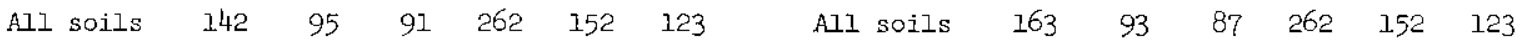

Note: $\mathrm{n}=$ number of samples; $\overline{\mathrm{x}}=$ mean or average; $\mathrm{s}=$ one standard deviation. 
Table 2

Mean and Standard Deviation Values for USCS and USDA Soil Types

Remolding Index, 6- to 12-in. Layer

\begin{tabular}{|c|c|c|c|c|c|c|c|}
\hline \multicolumn{4}{|c|}{ USCS } & \multicolumn{4}{|c|}{ USDA } \\
\hline Type & $n$ & $\overline{\bar{x}}$ & $s$ & Type & $\mathrm{n}$ & $\overline{\bar{x}}$ & $s$ \\
\hline \multicolumn{8}{|c|}{ High-'Topography, Wet-Season Condition } \\
\hline SM & 32 & 1.32 & 0.79 & LS & 12 & 1.62 & 0.96 \\
\hline $\mathrm{CH}$ & 11 & 1.04 & 0.16 & $C L$ & 5 & 1.19 & 0.12 \\
\hline MH & 9 & 1.02 & 0.36 & $\mathrm{SiC}$ & 2 & 1.16 & 0.06 \\
\hline SM-SC & 4 & 0.98 & 0.22 & $\mathrm{SL}$ & 29 & 1.04 & 0.57 \\
\hline $\mathrm{SC}$ & 10 & 0.94 & 0.27 & S & 4 & 1.03 & 0.48 \\
\hline CL-ML & 7 & 0.83 & 0.28 & SCL & 8 & 0.95 & 0.26 \\
\hline CI & 16 & 0.82 & 0.27 & SiL & 5 & 0.92 & 0.29 \\
\hline ML & 7 & 0.69 & 0.33 & C & 3 & 0.90 & 0.18 \\
\hline GM & 1 & 0.51 & -- & SiCL & 3 & 0.89 & 0.26 \\
\hline \multirow[t]{5}{*}{$\mathrm{GC}$} & 1 & 0.45 & -- & L & 23 & 0.83 & 0.28 \\
\hline & & & & GSiL & I & 0.71 & -- \\
\hline & & & & GSL & 1 & 0.51 & - \\
\hline & & & & GCI & 1 & 0.51 & -- \\
\hline & & & & GL & 1 & 0.45 & -- \\
\hline 11 soils & 98 & 1.03 & 0.55 & All soils & 98 & 1.03 & 0.55 \\
\hline
\end{tabular}

Low-Topography, Wet-Season Condition

\begin{tabular}{|c|c|c|c|c|c|c|c|}
\hline SP-SM & 6 & 1.31 & 0.68 & S & 9 & 1.34 & 0.69 \\
\hline SM & 55 & 0.89 & 0.57 & GSI & 2 & 1.14 & 0.02 \\
\hline $\mathrm{CH}$ & 101 & 0.88 & 0.24 & LS & 22 & 1.07 & 0.59 \\
\hline $\mathrm{MH}$ & 34 & 0.79 & 0.28 & $\mathrm{C}$ & 32 & 0.87 & 0.25 \\
\hline SC & 23 & 0.77 & 0.29 & Si & 1 & 0.86 & -- \\
\hline $\mathrm{CL}$ & 187 & 0.71 & 0.23 & SiL & 72 & 0.83 & 0.30 \\
\hline $\mathrm{OH}$ & 5 & 0.62 & 0.12 & SiCJ & 47 & 0.77 & 0.26 \\
\hline CL-ML & 24 & 0.57 & 0.21 & GSCL & 1 & 0.77 & -- \\
\hline ML & 35 & 0.51 & 0.27 & $\mathrm{SiC}$ & 39 & 0.76 & 0.19 \\
\hline \multirow[t]{5}{*}{ SM-SC } & 13 & 0.51 & 0.22 & CL & 38 & 0.75 & 0.20 \\
\hline & & & & $\mathrm{L}$ & 98 & 0.69 & 0.28 \\
\hline & & & & SL & 97 & 0.65 & 0.39 \\
\hline & & & & SCL & 26 & 0.64 & 0.16 \\
\hline & & & & GCL & 1 & 0.51 & 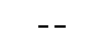 \\
\hline soils & 483 & 0.76 & 0.34 & All soils & 485 & 0.76 & 0.34 \\
\hline
\end{tabular}

Low-Topography, High-Moisture Condition

$\begin{array}{lrllllll}\text { SP-SM } & 2 & 1.22 & 0.56 & \text { S } & 5 & 1.32 & 0.69 \\ \text { CH } & 50 & 0.79 & 0.24 & \text { LS } & 9 & 0.86 & 0.65 \\ \text { MH } & 16 & 0.66 & 0.21 & \text { SiL } & 21 & 0.78 & 0.29 \\ \text { CL } & 91 & 0.65 & 0.20 & \text { C } & 15 & 0.75 & 0.14 \\ \text { OH } & 4 & 0.65 & 0.11 & \text { CL } & 21 & 0.73 & 0.22 \\ \text { SC } & 11 & 0.64 & 0.30 & \text { SiCL } & 29 & 0.71 & 0.27 \\ \text { SM } & 20 & 0.53 & 0.31 & \text { SiC } & 27 & 0.68 & 0.17 \\ \text { ML } & 17 & 0.43 & 0.22 & \text { SCL } & 15 & 0.60 & 0.15 \\ \text { CL-ML } & 12 & 0.42 & 0.13 & \text { L } & 50 & 0.59 & 0.22 \\ \text { SM-SC } & 3 & 0.34 & 0.21 & \text { SI } & 38 & 0.47 & 0.27 \\ \text { AlI soils } & 226 & 0.66 & 0.31 & \text { AII soils } & 230 & 0.66 & 0.31\end{array}$

Note: $\mathrm{n}=$ number of samples; $\overrightarrow{\mathrm{x}}=$ mean or average; $\mathrm{s}=$ one standard deviation. 
Table 3

Mean and Standard Deviation Values for USCS and USDA Soil Types

Rating Cone Index, 6- to 12-in. Layer

\begin{tabular}{|c|c|c|c|c|c|c|c|}
\hline & USCS & & & & USDA & & \\
\hline Type & $n$ & $\bar{x}$ & $S$ & Type & $\mathrm{n}$ & $\overline{\bar{x}}$ & s \\
\hline & & High- & raph & on Condition & & & \\
\hline $\mathrm{GC}$ & 1 & 253 & -- & LS & 12 & 325 & 268 \\
\hline SM & 32 & 227 & 197 & $\mathrm{SiC}$ & 2 & 274 & 16 \\
\hline $\mathrm{SC}$ & 10 & 221 & 165 & GL & I & 253 & -- \\
\hline SM-SC & 4 & 170 & 45 & CL & 5 & 245 & 89 \\
\hline $\mathrm{CH}$ & 11 & 169 & 81 & SCL & 8 & 221 & 193 \\
\hline CL & 16 & 150 & 125 & SL & 29 & 171 & 108 \\
\hline $\mathrm{MH}$ & 9 & 148 & 83 & $\mathrm{~s}$ & 4 & 145 & 69 \\
\hline CL-ML & 7 & 118 & 62 & C & 3 & 145 & 69 \\
\hline $\mathrm{ML}$ & 7 & 104 & 89 & SiCL & 3 & 136 & 124 \\
\hline GM & 1 & 89 & -- & $\mathrm{L}$ & 23 & 124 & 91 \\
\hline & & & & SiL & 5 & 99 & 47 \\
\hline & & & & GSiL & 1 & 89 & -- \\
\hline & & & & GCL & 1. & 89 & -- \\
\hline & & & & GSI & 1 & 53 & -- \\
\hline AlI soils & 98 & 180 & 148 & All soils & 98 & 180 & 147 \\
\hline & & Low- & graph & on Conditio & & & \\
\hline SP-SM & 6 & 234 & 184 & $\mathrm{~S}$ & 9 & 301 & 267 \\
\hline SM & 55 & 197 & 170 & GSL & 2 & 293 & 94 \\
\hline SC & 23 & 144 & 102 & IS & 22 & 230 & 164 \\
\hline CL-ML & 24 & 108 & 74 & $\mathrm{C}$ & 32 & 141 & 129 \\
\hline $\mathrm{CH}$ & 100 & 108 & 90 & SL & 97 & 121 & 123 \\
\hline $\mathrm{CI}$ & 187 & 102 & 60 & L & 98 & 119 & 116 \\
\hline $\mathrm{ML}$ & 34 & 101 & 91 & Si & 1 & 118 & $n$ \\
\hline $\mathrm{MH}$ & 33 & 90 & 57 & CL & 38 & 1.17 & 53 \\
\hline SM-SC & 13 & 85 & 53 & GSCL & 1. & 116 & 6 \\
\hline $\mathrm{OH}$ & 5 & 22 & 13 & SiI & 71 & 111 & 64 \\
\hline & & & & GCL & 26 & 91 & 56 \\
\hline & & & & SiCL & 46 & 84 & 52 \\
\hline & & & & SiC & 39 & 78 & 54 \\
\hline & & & & GCI & 1 & 53 & -- \\
\hline All soils & 480 & 121 & 1.11 & All soils & 483 & 120 & 111 \\
\hline & & Low-I & caphy & ture Condit & & & \\
\hline SP-SM & 2 & 132 & 98 & $\mathrm{~S}$ & 5 & 315 & 332 \\
\hline SM & 22 & 129 & 114 & LS & 9 & 134 & 75 \\
\hline $\mathrm{SC}$ & 11 & 113 & 106 & $\mathrm{CL}$ & 21 & 102 & 44 \\
\hline $\mathrm{ML}$ & 17 & 100 & 98 & SiL & 21 & 94 & 46 \\
\hline $\mathrm{CL}$ & 91 & 79 & 40 & C & 15 & 87 & 42 \\
\hline $\mathrm{CH}$ & 50 & 70 & 44 & $\mathrm{~L}$ & 50 & 85 & 69 \\
\hline $\mathrm{MH}$ & 16 & 67 & 36 & SCL & 15 & 79 & 42 \\
\hline CL-ML & 12 & 65 & 36 & SI & 38 & 75 & 82 \\
\hline$S M-S C$ & 3 & 47 & 37 & SiCL & 29 & 66 & 33 \\
\hline $\mathrm{OH}$ & 1. & 24 & 14 & $\mathrm{SiC}$ & 27 & 53 & 35 \\
\hline All soils & 228 & 86 & 81 & All soils & 230 & 86 & 81 \\
\hline
\end{tabular}

Note: $\mathrm{n}=$ number of samples; $\overline{\mathrm{x}}=$ mean or average; $\mathrm{s}=$ one standard deviation. 
Table 4

Mean and Standard Deviation Values for USCS and USDA Soil Types

Sheargraph Shear Strength in psi at 10-psi Normal Pressure

\begin{tabular}{|c|c|c|c|c|c|c|c|c|c|c|c|c|c|}
\hline \multirow[b]{3}{*}{ Type } & \multicolumn{6}{|c|}{ USCS } & \multicolumn{7}{|c|}{$\overline{\mathrm{USDA}}$} \\
\hline & \multicolumn{3}{|c|}{$\begin{array}{c}\text { Soil-to-Soil } \\
\text { Shear }\end{array}$} & \multicolumn{3}{|c|}{$\begin{array}{l}\text { Rubber-to-Soil } \\
\text { Shear }\end{array}$} & \multirow[b]{2}{*}{ Type } & \multicolumn{3}{|c|}{$\begin{array}{c}\text { Soil-to-soil } \\
\text { Shear }\end{array}$} & \multicolumn{3}{|c|}{$\begin{array}{c}\text { Rubber-to-Soil } \\
\text { Shear }\end{array}$} \\
\hline & $\mathrm{n}$ & $\bar{x}$ & $S$ & $n$ & $\overline{\mathrm{x}}$ & $s$ & & $\mathrm{n}$ & $\overline{\bar{x}}$ & 5 & $\mathrm{n}$ & $\overline{\bar{x}}$ & $s$ \\
\hline \multicolumn{14}{|c|}{ High-Topography, Wet-Season Condition } \\
\hline$C L$ & 4 & 8.88 & 1.23 & 4 & 7.45 & 0.85 & SiL & 1 & 9.00 & -- & 1 & 7.10 & -- \\
\hline $\mathrm{MH}$ & 4 & 8.88 & 1.90 & 4 & 6.43 & 1.11 & CL & 4 & 8.98 & 1.09 & 5 & 6.56 & 1.86 \\
\hline $\mathrm{CH}$ & 2 & 8.40 & 0.28 & 3 & 4.30 & 1.21 & L & 8 & 8.94 & 1.45 & 8 & 6.49 & 0.88 \\
\hline & 4 & 8.33 & 1.36 & 4 & 6.00 & 0.59 & $\mathrm{SiC}$ & 1 & 8.60 & -- & 1 & 5.70 & -- \\
\hline CL-ML & 3 & 8.13 & 1.95 & 3 & 6.70 & 0.96 & SiCL & 1 & 8.20 & -- & 1 & 3.60 & -- \\
\hline SM & 11 & 7.61 & 1.00 & 11 & 5.24 & 0.76 & SL & 7 & 7.80 & 0.97 & 7 & 5.91 & 0.61 \\
\hline SC & 2 & 7.20 & 0.99 & 2 & 5.90 & 1.13 & LS & 6 & 7.18 & 0.85 & 6 & 4.88 & 0.79 \\
\hline & & & & & & & SCL & 1 & 6.50 & -- & 1 & 5.10 & -- \\
\hline & & & & & & & & 1 & 6.20 & -- & 1 & 5.00 & -- \\
\hline All soils & 30 & 8.12 & 1.30 & 31 & 5.87 & 1.19 & A.l. soils & 30 & 8.12 & 1.30 & 31 & 5.87 & 1.19 \\
\hline \multicolumn{14}{|c|}{ Low-Topography, Wet-Season Condition } \\
\hline $\mathrm{CH}$ & 18 & 8.07 & 1.24 & 18 & 5.73 & 1.45 & SCL & 1 & 10.00 & -- & 1 & 6.90 & -- \\
\hline$C L$ & 46 & 7.77 & 2.13 & 42 & 6.24 & 1.58 & GCL & 1 & 9.40 & -- & 1 & 6.60 & -- \\
\hline $\mathrm{SM}-\mathrm{SC}$ & 5 & 7.46 & 1.16 & 5 & 5.16 & 0.86 & CL & 10 & 8.36 & 1.99 & 8 & 6.43 & 1.13 \\
\hline $\mathrm{SC}$ & 3 & 7.27 & 0.65 & 3 & 5.83 & 0.76 & SiL & 35 & 7.75 & 1.62 & 34 & 5.87 & 1.58 \\
\hline SM & 31 & 7.23 & 1.46 & 31 & 5.25 & 1.04 & SL & 42 & 7.59 & 1.32 & 42 & 5.37 & 1.02 \\
\hline SP-SM & 1 & 7.10 & -- & 1 & 5.70 & -- & I & 24 & 7.15 & 2.22 & 23 & 5.51 & 1.27 \\
\hline ML & 18 & 7.06 & 1.95 & 17 & 5.26 & 1.09 & $\mathrm{~S}$ & 1 & 7.10 & - & 1 & 5.70 & -- \\
\hline CL-ML & 8 & 6.76 & 2.39 & 7 & 5.34 & 1.28 & SiCL & 17 & 7.04 & 2.28 & 15 & 6.25 & 1.65 \\
\hline \multirow[t]{4}{*}{$\mathrm{MH}$} & 10 & 6.02 & 1.88 & 7 & 5.31 & 1.01 & $\mathrm{C}$ & 5 & $\begin{array}{l}6.98 \\
632\end{array}$ & 2.26 & 3 & 5.83 & 0.97 \\
\hline & & & & & & & LS & & 0.33 & & & 4.98 & 0.63 \\
\hline & & & & & & & GSL & 1 & 4.00 & - & 1 & 4.40 & -- \\
\hline & & & & & & & $\mathrm{SiC}$ & 2 & 3.65 & 2.33 & 1 & $3 \cdot 30$ & -- \\
\hline All soils & 140 & 7.39 & 1.88 & 131 & 5.66 & 1.35 & All soils & 151 & $7 \cdot 37$ & 1.84 & 142 & 5.64 & 1.32 \\
\hline \multicolumn{14}{|c|}{ Low-Topography, High-Moisture Condition } \\
\hline $\mathrm{CH}$ & 4 & 8.85 & 0.82 & 4 & 6.43 & 0.13 & GCL & 1 & 8.20 & -- & 1 & 7.40 & -- \\
\hline CL-ML & 4 & 7.00 & 3.36 & 3 & 5.57 & 2.61 & SiL & 10 & 7.53 & 2.66 & 9 & 6.02 & 1.66 \\
\hline & 6 & 6.68 & 1.35 & 6 & 4.90 & 0.49 & SL & 5 & 6.86 & 1.36 & 5 & 4.62 & 0.92 \\
\hline CL & 16 & 6.67 & 3.11 & 12 & 5.68 & 1.81 & CL & 5 & 6.84 & 3.54 & 3 & 5.17 & 3.00 \\
\hline $\mathrm{ML}$ & 5 & 4.84 & 2.03 & 4 & 4.35 & 1.49 & $\mathrm{C}$ & 3 & 6.33 & 3.00 & 1 & 6.40 & - \\
\hline $\mathrm{MH}$ & 3 & 3.80 & 1.85 & -- & -- & -- & LS & 4 & 5.93 & 0.77 & 4 & 4.73 & 0.51 \\
\hline & & & & & & & L & 11 & 5.93 & 2.06 & 10 & 4.68 & 0.92 \\
\hline & & & & & & & $\begin{array}{l}\text { SiCL } \\
\text { SiC }\end{array}$ & $\begin{array}{l}4 \\
1\end{array}$ & $\begin{array}{l}5.23 \\
2.00\end{array}$ & $\begin{array}{l}4.09 \\
--\end{array}$ & 2 & 6.70 & 0.42 \\
\hline All soils & 38 & 6.47 & 2.68 & 29 & 5.43 & 1.57 & All soils & 44 & 6.43 & 2.52 & 35 & 5.31 & 1.46 \\
\hline
\end{tabular}

Note: $\mathrm{n}=$ number of samples; $\overrightarrow{\mathrm{x}}=$ mean or average; $\mathrm{s}=$ one standard deviation. 
Table 5

Mean and Standard Deviation Values for USCS and USDA Soil Types

Moisture Content, \% Dry Weight, 0- to 6-in. and 6- to 12-in. Layers

\begin{tabular}{|c|c|c|c|c|c|c|c|c|c|c|c|c|c|}
\hline \multicolumn{7}{|c|}{ ÚSCS } & \multicolumn{7}{|c|}{ USDA } \\
\hline \multirow[b]{2}{*}{ Type } & \multirow{2}{*}{$\frac{0-\text { to }}{n}$} & \multirow{2}{*}{$\frac{6-i n .}{\bar{x}}$} & \multirow{2}{*}{$\frac{\text { Layer }}{\mathrm{s}}$} & \multicolumn{3}{|c|}{$\begin{array}{l}6 \text { - to } 12-\text { in. } \\
\text { Layer }\end{array}$} & \multirow[b]{2}{*}{ Type } & \multicolumn{2}{|c|}{0 - to 6 -in. } & \multirow{2}{*}{$\frac{\text { Layer }}{\mathrm{s}}$} & \multicolumn{3}{|c|}{$\begin{array}{l}\text { 6- to } 12-i n . \\
\text { Layer }\end{array}$} \\
\hline & & & & $\mathrm{n}$ & $\overline{\bar{x}}$ & $\mathrm{~s}$ & & $\mathrm{n}$ & $\overline{\mathrm{x}}$ & & $\mathrm{n}$ & $\overline{\bar{x}}$ & $\mathrm{~s}$ \\
\hline & & & & $\underline{\mathrm{Hi}}$ & -Topos & phy, & Seasor & aditj & & & & & \\
\hline SP-SM & 1 & 11.0 & -- & - & -- & -- & LS & 16 & 9.1 & 3.8 & 19 & 11.1 & 4.6 \\
\hline SM-SC & 3 & 16.5 & 2.6 & 8 & 13.0 & 3.1 & $\mathrm{~s}$ & 5 & 8.5 & 1.0 & 5 & 12.2 & 6.7 \\
\hline & 39 & 12.9 & 6.5 & 49 & 13.4 & 7.3 & GL & 2 & 18.3 & 0.4 & 1 & 14.0 & -- \\
\hline & $\cdots$ & -- & -- & 1 & 14.0 & -- & $\mathrm{SC}$ & -- & -- & -- & 2 & 14.1 & 8.5 \\
\hline $\mathrm{CL}-\mathrm{ML}$ & 3 & 15.9 & 1.1 & 8 & 15.8 & 3.3 & GSL & 1 & 11.0 & -- & 5 & $i 4.6$ & 3.3 \\
\hline SC & 7 & 20.5 & 5.1 & 14 & 18.0 & 4.5 & GSCL & 1 & 17.4 & - & -- & -- & - \\
\hline ML & 13 & 19.0 & 5.9 & 11 & 18.6 & 8.2 & SL & 34 & 19.7 & 9.7 & 43 & 16.9 & 9.1 \\
\hline CL & 8 & 22.7 & 5.0 & 20 & 18.8 & 4.6 & GSiL & -- & - & -- & 1 & 17.2 & -- \\
\hline GM & -- & -- & $=-$ & 1 & 22.4 & -- & SCL & 5 & 19.9 & 9.8 & 8 & 18.8 & 4.4 \\
\hline $\mathrm{CH}$ & 7 & 31.4 & 6.0 & 14 & 30.9 & 5.4 & GCL & -- & -- & -- & 2 & 19.4 & 4.2 \\
\hline MH & 17 & 41.6 & 8.5 & 14 & 39.1 & 8.3 & L & 17 & 27.5 & 13.6 & 27 & 23.8 & 11.5 \\
\hline & & & & & & & SiL & 10 & 33.4 & 10.2 & 6 & 24.5 & 8.2 \\
\hline & & & & & & & $\mathrm{C}$ & 2 & 23.3 & 10.3 & 6 & 28.6 & 8.3 \\
\hline & & & & & & & $\mathrm{CL}$ & 8 & 28.5 & 11.3 & 7 & 29.1 & 9.7 \\
\hline & & & & & & & SiC & 2 & 31.8 & 4.5 & 2 & 31.1 & 3.4 \\
\hline & & & & & & & SiCL & 1 & 35.4 & -- & 4 & 37.3 & 6.1 \\
\hline il soils & 98 & 21.5 & 12.2 & 140 & 19.6 & 10.4 & All so: & 104 & 21.1 & 12.0 & 138 & 19.5 & 10.3 \\
\hline
\end{tabular}

Low-Topography, Wet-Season Condition

\begin{tabular}{|c|c|c|c|c|c|c|c|c|c|c|c|c|c|}
\hline $\mathrm{GC}$ & -- & -- & -- & 1 & 12.6 & -- & LS & 28 & 15.5 & 5.7 & 37 & 14.6 & 4.7 \\
\hline$S M$ & 58 & 15.5 & 6.5 & 90 & 15.6 & 6.1 & GSCL & -- & -- & -- & 2 & 15.4 & 4.0 \\
\hline CI-ML & 11 & 19.9 & 3.6 & 40 & 16.1 & 5.1 & GSICI & - - & -- & -- & 1 & 15.7 & -- \\
\hline$S M-S C$ & 7 & 17.5 & 10.2 & 20 & 16.2 & 4.4 & GSI & 2 & 16.9 & 2.1 & 4 & 16.1 & 4.2 \\
\hline SP-SM & 1 & 18.5 & -- & 9 & 16.4 & 7.3 & GL & -- & -- & -- & 1 & 17.5 & - \\
\hline SC & 10 & 17.1 & 5.9 & 34 & 18.5 & 9.9 & GCL & 1 & 17.4 & -- & 4 & 18.0 & 8.4 \\
\hline ML & 52 & 22.2 & 10.0 & 49 & 18.5 & 6.9 & $\mathrm{~S}$ & 4 & 15.5 & 11.8 & 14 & 18.3 & 7.3 \\
\hline CL & 81 & 23.4 & 7.0 & 231 & 22.5 & 7.3 & SL & 83 & 18.0 & 8.5 & 139 & 18. & 12.0 \\
\hline $\mathrm{CH}$ & 50 & 36.6 & 10.8 & 113 & 35.5 & 15.8 & SCL & 3 & 25.2 & 16.3 & 34 & 18.5 & 4.5 \\
\hline MH & 26 & 46.0 & 20.4 & 34 & 45 & 20.5 & L & 56 & 25.9 & 16.2 & 118 & 22.7 & 12.5 \\
\hline $\mathrm{OH}$ & 8 & 66.2 & 20.1 & 6 & 93.4 & 11.9 & SiL & 79 & 28.5 & 12.7 & 93 & 27.1 & 12.8 \\
\hline & & & & & & & $\mathrm{CL}$ & 14 & 31.6 & 15.3 & 51 & 27. & 19.6 \\
\hline & & & & & & & C & 22 & 39.0 & 13.4 & 39 & 32.6 & 7.9 \\
\hline & & & & & & & Si & 4 & 23.8 & 8.0 & 2 & 33.5 & 12.6 \\
\hline & & & & & & & SiCL & 32 & 31.7 & 10.6 & 50 & 3 & 17.7 \\
\hline & & & & & & & SiC & 70 & 48.7 & 18.2 & 35 & & 19.4 \\
\hline soil & ol & 26.5 & 15.2 & 627 & 24. & 15.0 & All soils & 347 & 26.4 & 14.8 & 624 & 24.6 & 15. \\
\hline
\end{tabular}
Low-Topography, High-Moisture Condition

\begin{tabular}{|c|c|c|c|c|c|c|c|c|c|c|c|c|c|}
\hline CL-ML & 7 & 21.2 & 4.6 & 16 & 17.9 & 4.7 & LS & 8 & 18.3 & 6.9 & 12 & 18.3 & 5.1 \\
\hline$S M$ & 16 & 16.6 & 6.1 & 30 & 18.0 & 5.5 & SCL & -- & -- & -- & 15 & 18.3 & 5.0 \\
\hline$S M-S C$ & 1 & 16.6 & - & 4 & 20.0 & 5.0 & GCL & 1 & 17.4 & -- & -- & -- & $\therefore$ \\
\hline $\mathrm{SC}$ & 2 & 20.0 & 7.0 & 12 & 20.7 & 3.8 & GSL & -- & -- & -- & 1 & 20.4 & -- \\
\hline MI & 25 & 24.2 & 7.7 & 17 & 22.0 & 8.3 & SL & 25 & 19.9 & 5.3 & 41 & 22.6 & 16.9 \\
\hline SP-SM & 1 & 16.8 & - & 2 & 24.6 & 2.3 & Si & 1 & 27.7 & - & -- & -- & - \\
\hline CL & 35 & 26.4 & 5.5 & 93 & 24.8 & 7.0 & S & 3 & 17.0 & 13.4 & 6 & 23.1 & 5.9 \\
\hline $\mathrm{CH}$ & 18 & 44.3 & 12.8 & 44 & 39.6 & 15.7 & $\mathrm{~L}$ & 30 & 24.4 & 6.0 & 53 & 24.3 & 11.7 \\
\hline MH & 13 & 46.9 & 19.9 & 14 & 52.7 & 27.3 & SiL & 18 & 27.9 & 7.9 & 21 & 26.0 & 9.0 \\
\hline \multirow[t]{4}{*}{$\mathrm{OH}$} & 4 & 69.6 & 12.9 & 4 & 92.3 & 14.9 & CL & 12 & 33.2 & 13.7 & 22 & 28.4 & 12.5 \\
\hline & & & & & & & C & 14 & 41.3 & 15.4 & 16 & 34.0 & 7.1 \\
\hline & & & & & & & SICL & 12 & 38.1 & 13.4 & 26 & 39.4 & 21.7 \\
\hline & & & & & & & SiC & 16 & 52.7 & 18.1 & 23 & 49.0 & 21.8 \\
\hline soil & 122 & 30.3 & 15.7 & 236 & 28.5 & 16.8 & All soils & 140 & 30.3 & 15.1 & 236 & 28.5 & 16.8 \\
\hline
\end{tabular}


Table 6

Mean and Standard Deviation Values for USCS and USDA Soil Types

Dry Density, lb per cu ft, 6- to 12-in. Layer

\begin{tabular}{|c|c|c|c|c|c|c|c|}
\hline \multicolumn{4}{|c|}{ USCS } & \multicolumn{4}{|c|}{ USDA } \\
\hline Type & $\mathrm{n}$ & $\bar{x}$ & $\mathrm{~s}$ & Type & $n$ & $\overline{\mathrm{x}}$ & $S$ \\
\hline \multicolumn{8}{|c|}{ High-Topography, Wet-Season Condition } \\
\hline SM-SC & 5 & 112.2 & 8.2 & GSL & 3 & 109.5 & 13.2 \\
\hline GC & 1 & 104.8 & $-\infty$ & GL & 1 & 104.8 & - \\
\hline MI & 3 & 102.0 & 2.7 & SI & 27 & 102.7 & 5.8 \\
\hline CL-ML & 8 & 101.3 & 5.8 & S & 5 & 96.1 & 8.8 \\
\hline SM & 37 & 98.3 & 6.5 & L & 14 & 98.7 & 5.0 \\
\hline CL & 13 & 96.6 & 6.7 & SCL & 8 & 95.3 & 11.5 \\
\hline $\mathrm{SC}$ & 9 & 95.8 & 11.1 & GCL & 1 & 94.6 & -- \\
\hline $\mathrm{GM}$ & 1 & 94.6 & -- & LS & 14 & 94.4 & 3.0 \\
\hline $\mathrm{CH}$ & 5 & 82.4 & 6.6 & SiL & 1 & 91.8 & -- \\
\hline \multirow[t]{4}{*}{$\mathrm{MH}$} & 7 & 75.1 & 10.9 & $\mathrm{C}$ & 2 & 85.6 & 2.0 \\
\hline & & & & SICL & 4 & 79.1 & 10.7 \\
\hline & & & & CL & 6 & 78.9 & 12.1 \\
\hline & & & & $\mathrm{SiC}$ & 2 & 75.5 & 1.7 \\
\hline Al1 soils & 89 & 96.2 & 10.9 & AIl soils & 88 & 96.0 & 10.6 \\
\hline \multicolumn{8}{|c|}{ Low-Topography, Wet-Season Condition } \\
\hline$S M-S C$ & 12 & 101.9 & 5.1 & GSiCL & 1 & 117.3 & -- \\
\hline $\mathrm{SC}$ & 13 & 100.3 & $7 \cdot 5$ & GCI & 1 & 110.4 & -- \\
\hline SM & 48 & 99.5 & 7.7 & GSCL & 1 & 107.9 & -- \\
\hline CL-MI & 23 & 99.4 & 8.4 & GSI & 2 & 103.4 & 13.5 \\
\hline ML & 29 & 97.7 & 7.9 & SCI & 23 & 100.5 & 7.2 \\
\hline $\mathrm{CL}$ & 135 & 97.1 & 8.2 & SL & 72 & 99.9 & 7.6 \\
\hline SP-SM & 6 & 92.0 & 5.7 & LS & 22 & 98.9 & 5.0 \\
\hline $\mathrm{CH}$ & 54 & 80.5 & 10.3 & I & 73 & 97.3 & 10.4 \\
\hline $\mathrm{MH}$ & 8 & 69.9 & 10.5 & CL & 26 & 96.5 & 6.5 \\
\hline \multirow[t]{5}{*}{$\mathrm{OH}$} & 4 & 47.7 & 4.7 & SiI & 22 & 94.2 & 7.9 \\
\hline & & & & $S$ & 10 & 90.9 & 5.4 \\
\hline & & & & SiCL & 21 & 85.4 & 15.4 \\
\hline & & & & $\mathrm{C}$ & 25 & 79.6 & $7 \cdot 5$ \\
\hline & & & & $\mathrm{SIC}$ & 32 & 77.4 & 14.2 \\
\hline All soils & 332 & 93.9 & 12.4 & All soils & 331 & 93.9 & 12.4 \\
\hline
\end{tabular}

Note: $\mathrm{n}=$ number of samples; $\overline{\mathrm{x}}=$ mean or average; $\mathrm{s}=$ one standard deviation. 
Table 7

Mean and Standard Deviation Values for USCS and USDA Soil Types

Specific Gravity, 6- to 12-in. Layer

\begin{tabular}{|c|c|c|c|c|c|c|c|}
\hline \multicolumn{4}{|c|}{ USCS } & \multicolumn{4}{|c|}{ USDA } \\
\hline Type & $n$ & $\overline{\mathrm{x}}$ & $S$ & Type & $\underline{n}$ & $\overline{\bar{x}}$ & $s$ \\
\hline \multicolumn{8}{|c|}{ High-Topography, Wet-Season Condition } \\
\hline GM & 2 & 3.10 & 0.28 & $\mathrm{GL}$ & 5 & 2.97 & 0.28 \\
\hline $\mathrm{GC}$ & I & 2.96 & -- & GSCL & 2 & 2.91 & 0.37 \\
\hline $\mathrm{MH}$ & 12 & 2.77 & 0.11 & GSL & 8 & 2.90 & 0.30 \\
\hline $\mathrm{SC}$ & 15 & 2.77 & 0.24 & GCL & 2 & 2.81 & 0.15 \\
\hline SM-SC & 12 & 2.71 & 0.20 & SiCL & 2 & 2.77 & 0.11 \\
\hline $\mathrm{CH}$ & 12 & 2.69 & 0.06 & $C L$ & 7 & 2.75 & 0.11 \\
\hline CL & 18 & 2.68 & 0.03 & $\mathrm{~L}$ & 19 & 2.69 & 0.06 \\
\hline SM & 40 & 2.65 & 0.10 & GSiL & 1 & 2.68 & -- \\
\hline $\mathrm{ML}$ & 7 & 2.64 & 0.02 & SiC & 2 & 2.68 & 0.02 \\
\hline \multirow[t]{5}{*}{$\mathrm{CL}-\mathrm{ML}$} & 3 & 2.64 & 0.02 & SiL & 5 & 2.67 & 0.08 \\
\hline & & & & $\begin{array}{l}\mathrm{SI} \\
\mathrm{C}\end{array}$ & 39 & $\begin{array}{l}2.65 \\
2.65\end{array}$ & $\begin{array}{l}0.07 \\
0.05\end{array}$ \\
\hline & & & & S & 1 & 2.64 & -- \\
\hline & & & & IS & 18 & 2.63 & 0.02 \\
\hline & & & & SCL & 5 & 2.63 & 0.03 \\
\hline All soils & 122 & 2.70 & 0.14 & All soils & 122 & 2.70 & 0.14 \\
\hline
\end{tabular}

Low-Topography, Wet-Season Condition

\begin{tabular}{|c|c|c|c|c|c|c|c|}
\hline $\mathrm{CH}$ & 86 & 2.68 & 0.07 & GSiCL & 1 & 3.00 & -- \\
\hline $\mathrm{SC}$ & 20 & 2.67 & 0.11 & GL & 2 & 2.98 & 0.23 \\
\hline $\mathrm{MH}$ & 34 & 2.66 & 0.08 & GSI & 5 & 2.79 & 0.1 .1 \\
\hline CI & 128 & 2.66 & 0.06 & GCL & 3 & 2.76 & 0.05 \\
\hline $\mathrm{CL}-\mathrm{ML}$ & 24 & 2.65 & 0.04 & GSCI & $\hat{1}$ & 2.73 & -- \\
\hline SM & 65 & 2.65 & 0.07 & Si & 2 & 2.71 & 0.03 \\
\hline SP-SM & 4 & 2.65 & 0.06 & SiC & 25 & 2.71 & 0.04 \\
\hline $\mathrm{SM}-\mathrm{SC}$ & 8 & 2.65 & 0.05 & CL & 26 & 2.68 & 0.05 \\
\hline ML & 33 & 2.64 & 0.05 & $C$ & 26 & 2.67 & 0.07 \\
\hline \multirow[t]{7}{*}{$\mathrm{OH}$} & 6 & 2.61 & 0.18 & $\mathrm{SCL}$ & 13 & 2.65 & 0.03 \\
\hline & & & & SiCL & 36 & 2.65 & 0.08 \\
\hline & & & & SiI & 79 & 2.65 & 0.07 \\
\hline & & & & I & 65 & 2.65 & 0.07 \\
\hline & & & & S & 8 & 2.64 & 0.05 \\
\hline & & & & LS & 28 & 2.64 & 0.02 \\
\hline & & & & SL & 87 & 2.64 & 0.04 \\
\hline soils & 408 & 2.66 & 0.07 & All soils & 407 & 2.66 & 0.07 \\
\hline
\end{tabular}

Note: $\mathrm{n}=$ number of samples; $\overline{\mathrm{x}}=$ mean or average; $\mathrm{s}=$ one standard deviation. 



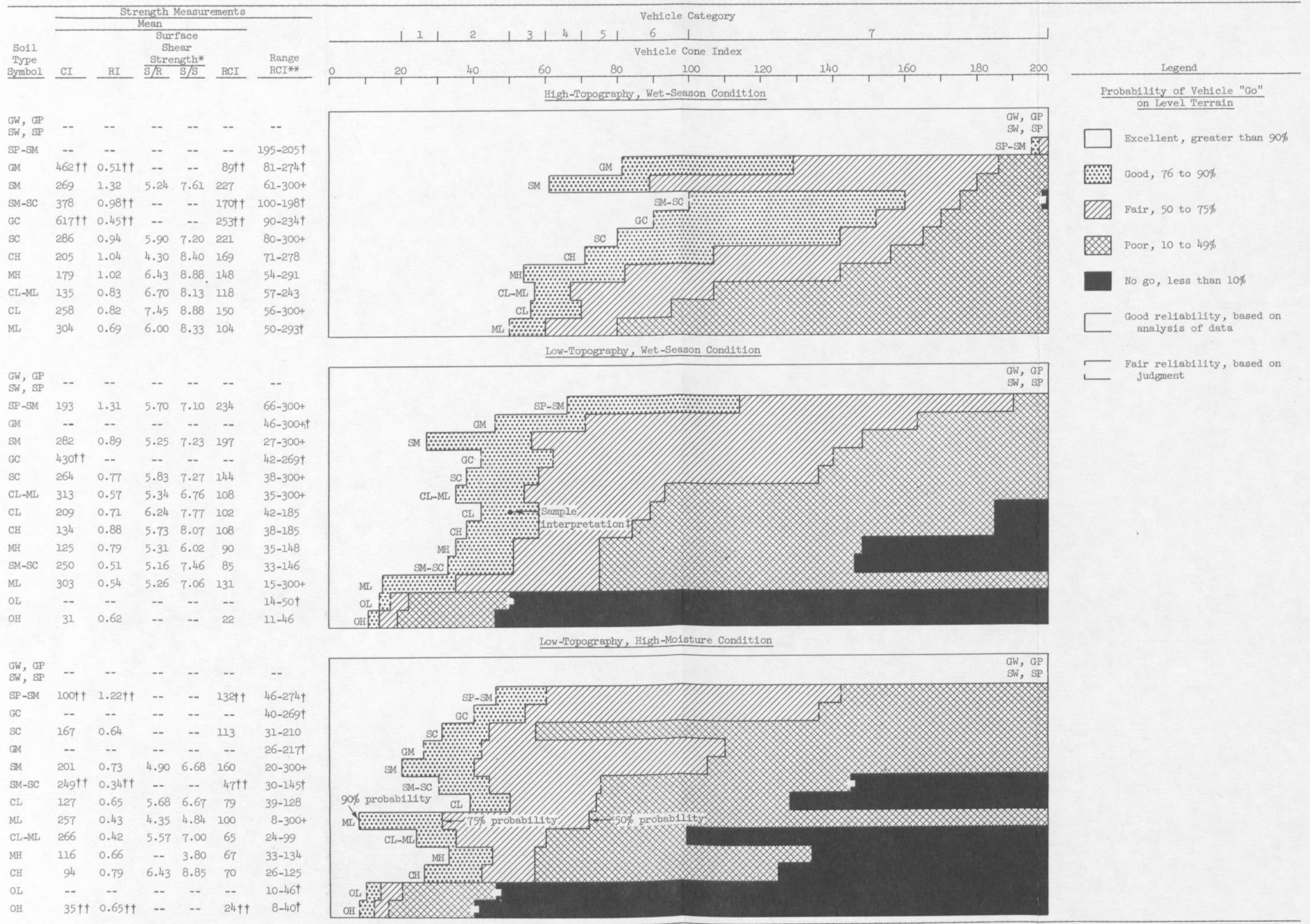

Vote: Vehicle category and cone index range are given in paragraph 52 .
* Sheargraph soil-to-rubber $(\mathrm{S} / \mathrm{R})$ and soil-to-soil $(\mathrm{S} / \mathrm{S})$ strength in psi for a normal load of 10 psi.

*
* Excluding lowest loo and highest $10 \%$ of all RCI values.
F

t十 Based on analysis of less than frive samples.
f A vehicle with a vehicle cone index of 50 would have a $75-90 \%$ chance of "go" on a CL soil of low-topography, wet-season condition. 

Table 9

So11 Trafficability Classification in USDA Terms

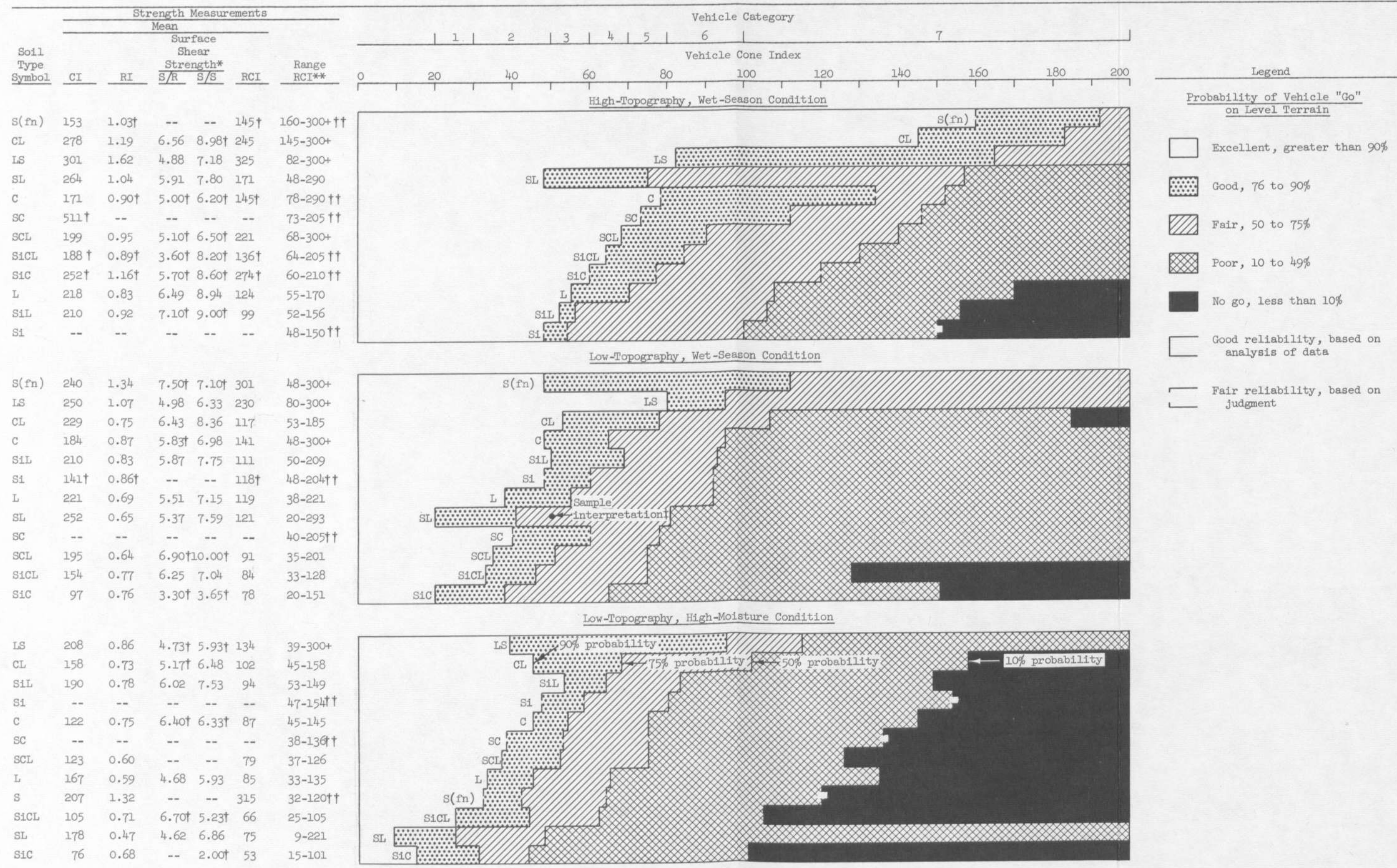

\footnotetext{
Note: Vehicle category and cone index range are given in paragraph 52 .
* Sheargraph soli-to-rubber ( $\mathrm{S} / \mathrm{R}$ ) and soil-to-soil ( $\mathrm{S} / \mathrm{S}$ ) strength in psi for a normal load of $10 \mathrm{psi}$.

* Excluding lowest 10\% and highest 10\% of all RCI values.

Estimated from textural, plasticity, and organic properties of soil under given moisture condition.
1 A vehicle with a vehicle cone 1 ndex of 50 would have a $50-75 \%$ chance of "go" on an SL soil of low-topography, wet-season condition.
} 



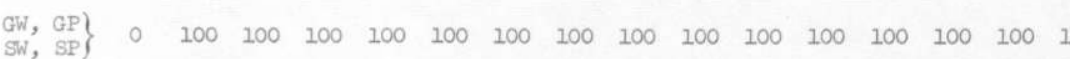

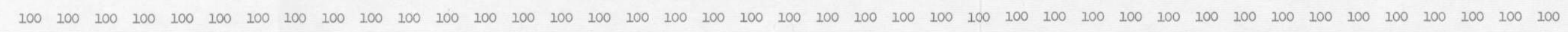

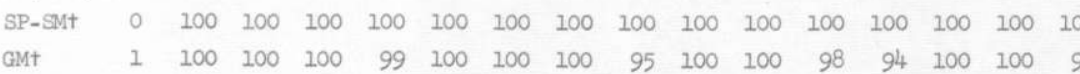
$\begin{array}{lrlllllllllllllll} & 32 & 97 & 96 & 95 & 93 & 97 & 96 & 94 & 88 & 95 & 94 & 92 & 86 & 95 & 94 & 90\end{array}$

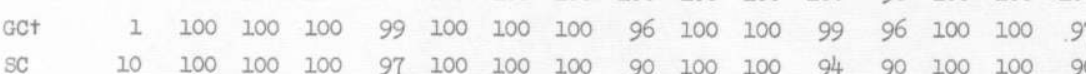

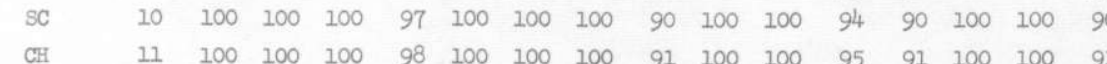
(fif $\quad \begin{array}{lllllllllllllll}100 & 100 & 100 & 95 & 100 & 100 & 100 & 78 & 100 & 100 & 88 & 78 & 100 & 100 & 78\end{array}$

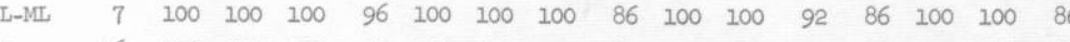

$\begin{array}{llllllllllllllll}16 & 100 & 100 & 100 & 92 & 100 & 100 & 97 & 82 & 100 & 95 & 91 & 80 & 100 & 94 & 88 \\ \text { Lit } & 100 & 100 & 100 & 87 & 100 & 100 & 99 & 68 & 100 & 95 & 83 & 65 & 100 & 90 & 75\end{array}$

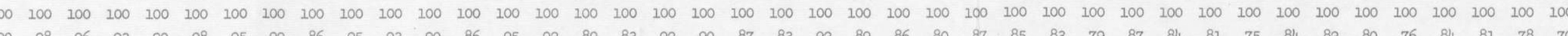

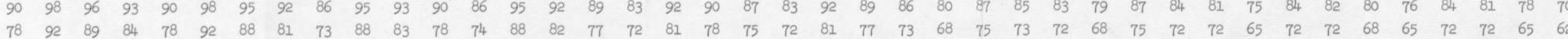

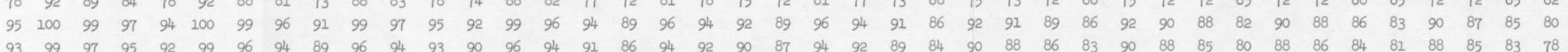

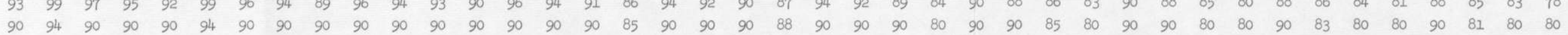

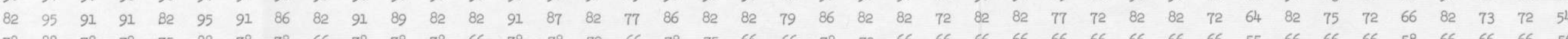
\begin{tabular}{lllllllllllllllllllllllll}
78 & 66 & 78 & 78 & 78 & 66 & 78 & 78 & 72 & 66 & 78 & 75 & 66 & 66 & 78 & 73 & 66 & 66 & 66 & 66 & & & \\
\hline
\end{tabular} 



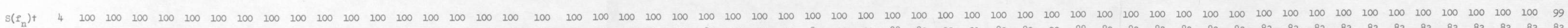

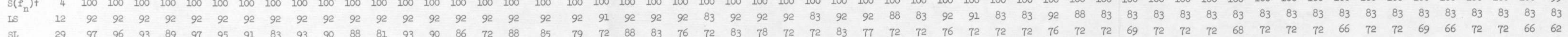

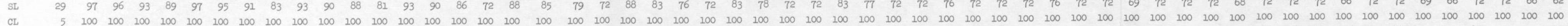

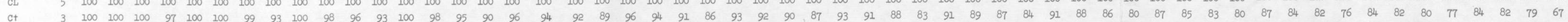

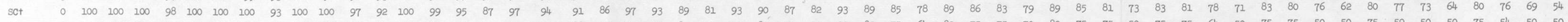

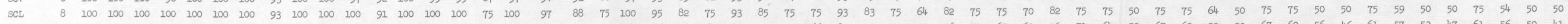

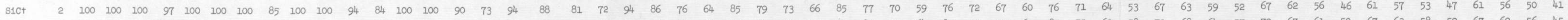

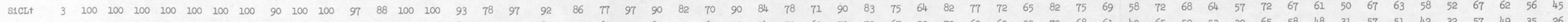

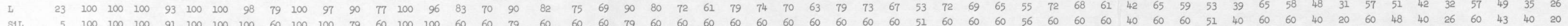

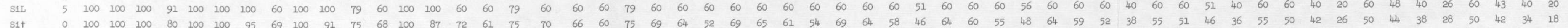

\author{
Lov-Topography, wet-Season condition
}

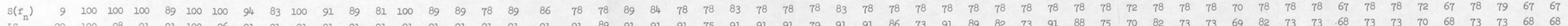

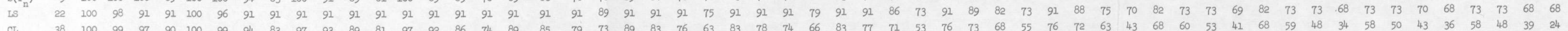

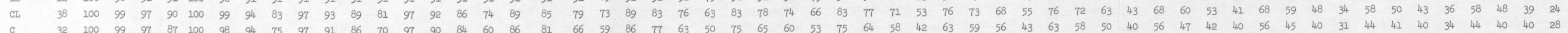

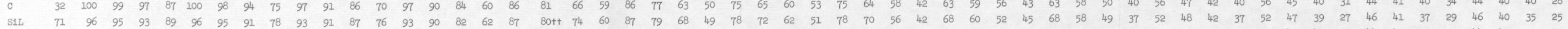

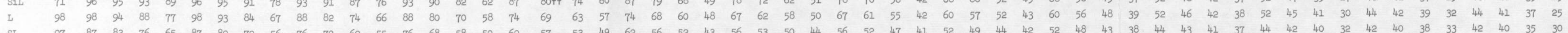

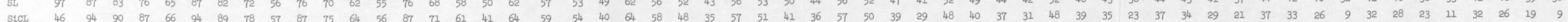

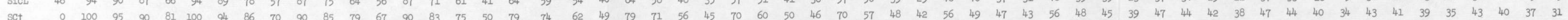

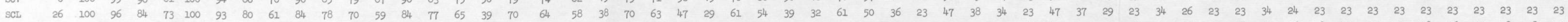

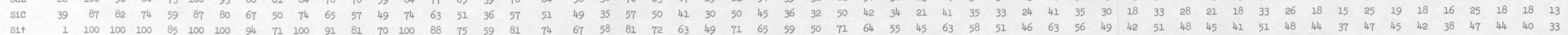

\title{
Low-Topography, High-Moisture Condition
}

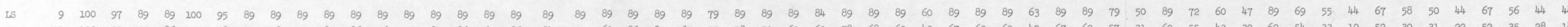

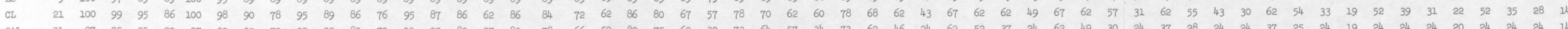

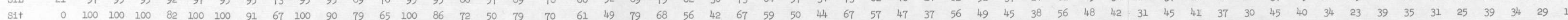

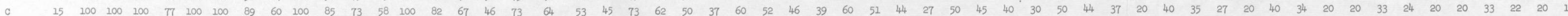

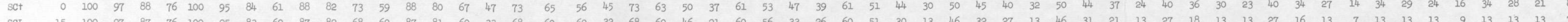

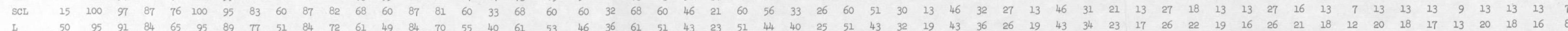

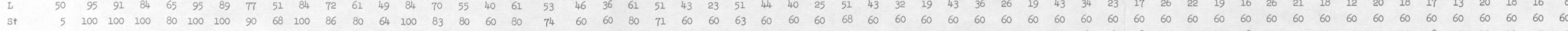

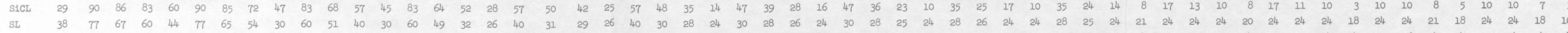

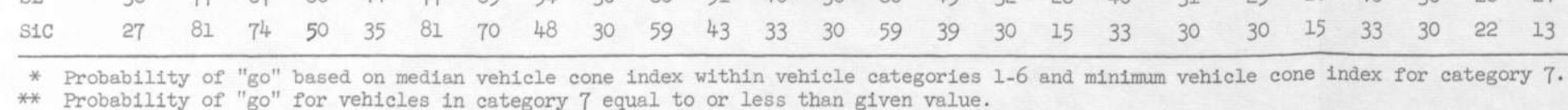

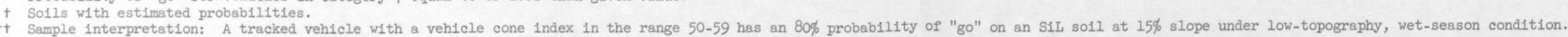





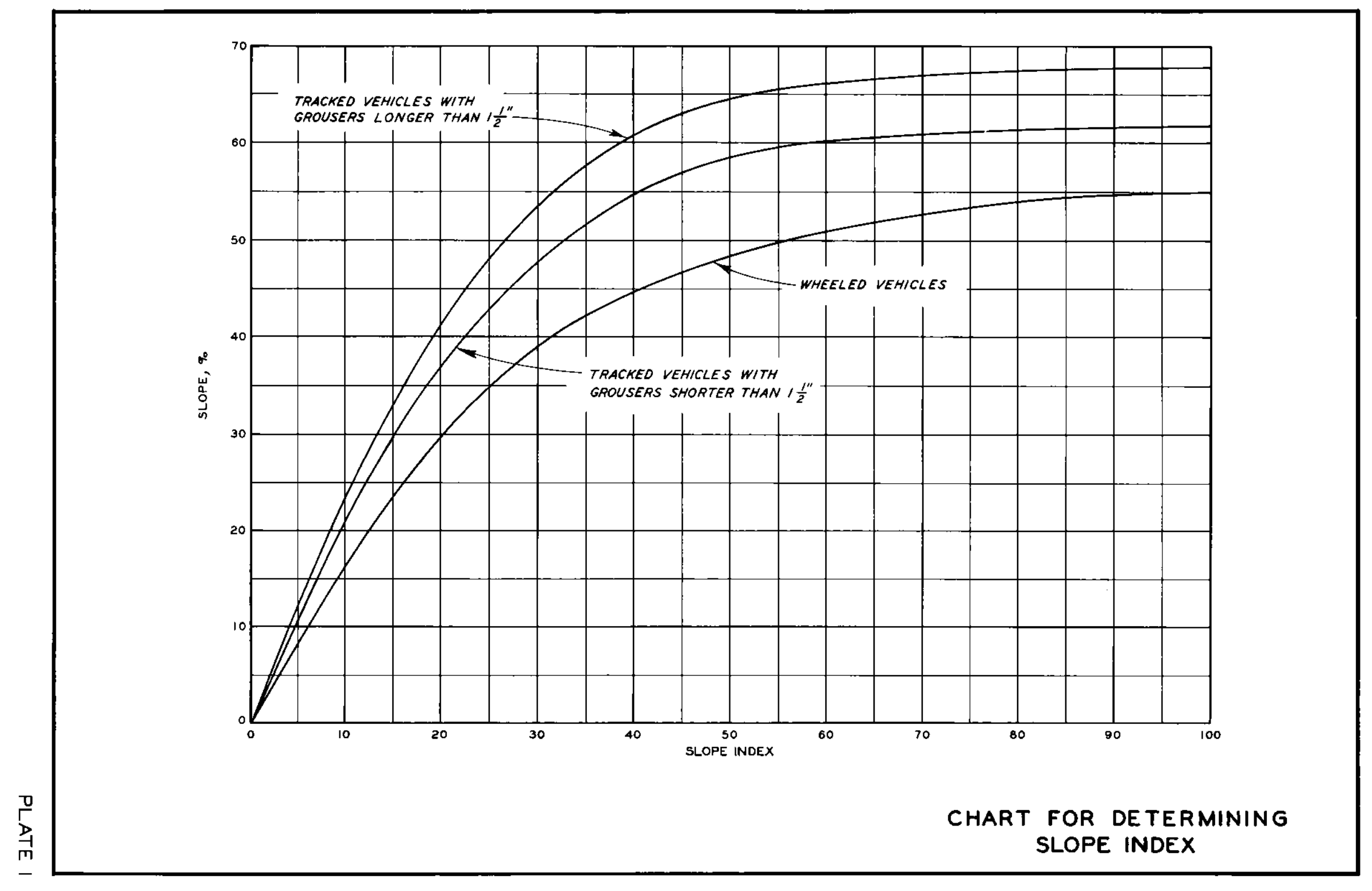




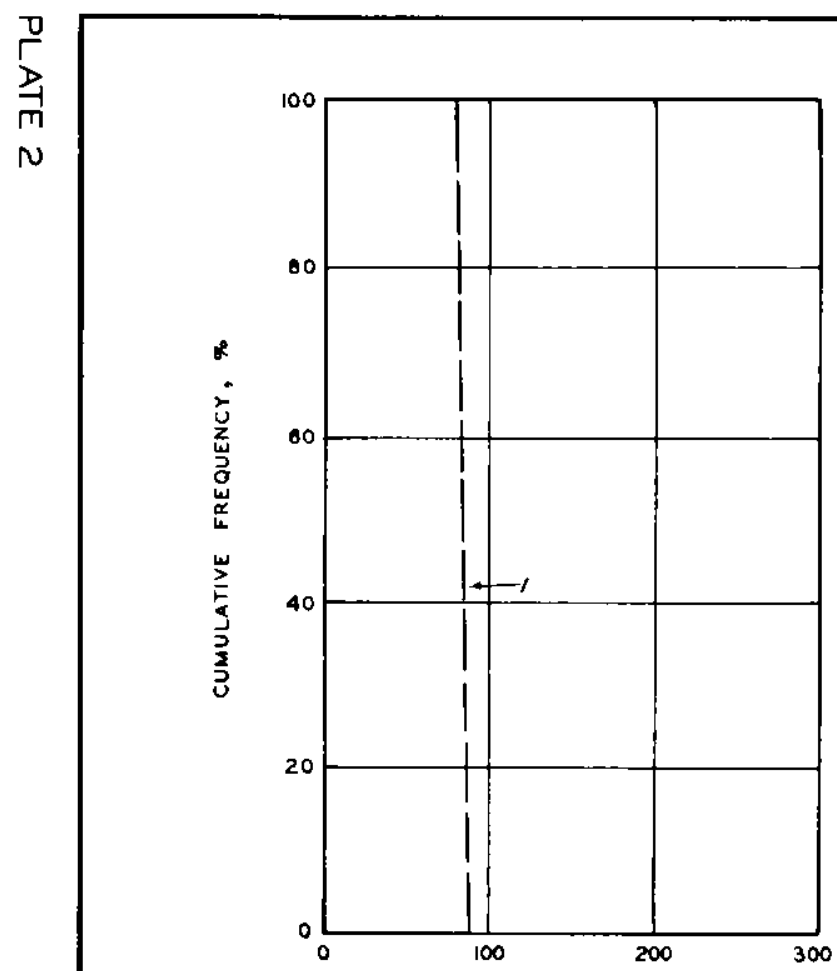

$\underline{G M}$

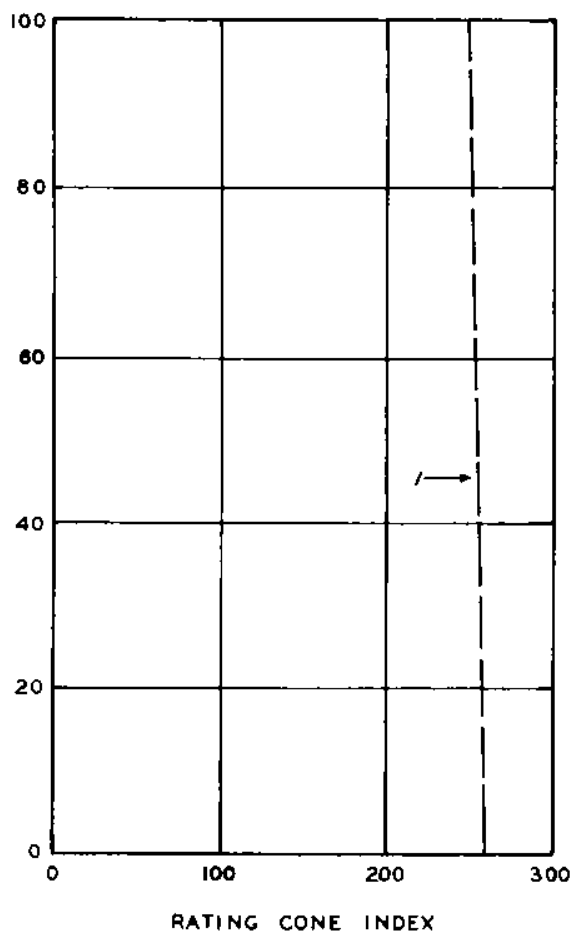

$\underline{\mathrm{GC}}$

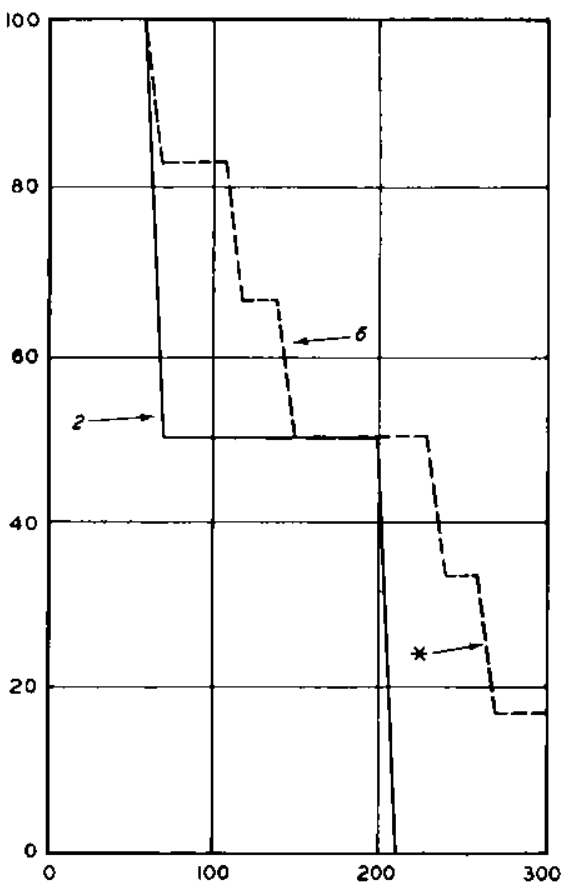

$\underline{S P-S M}$

\section{LEGEND}

LOW-TOPOGRAPHY, HIGH-MOISTURE CONOITION LOW-TOPOGRAPHY,

WET-SEASON CONDITION HIGH-TOPOGRAPHY,
NOTE: NUMBERS OY CURVES DENOTE NUMBER OF SAMPLES USED IN ANALYSIS

* SAMPLE INTERPRETATION: 25\% OF SAMPLES OCCURRED 265 OR HIGHER.
CUMULATIVE FREQUENCY OF RATING CONE INDEX USCS SOILS $G M, G C, A N D S P-S M$




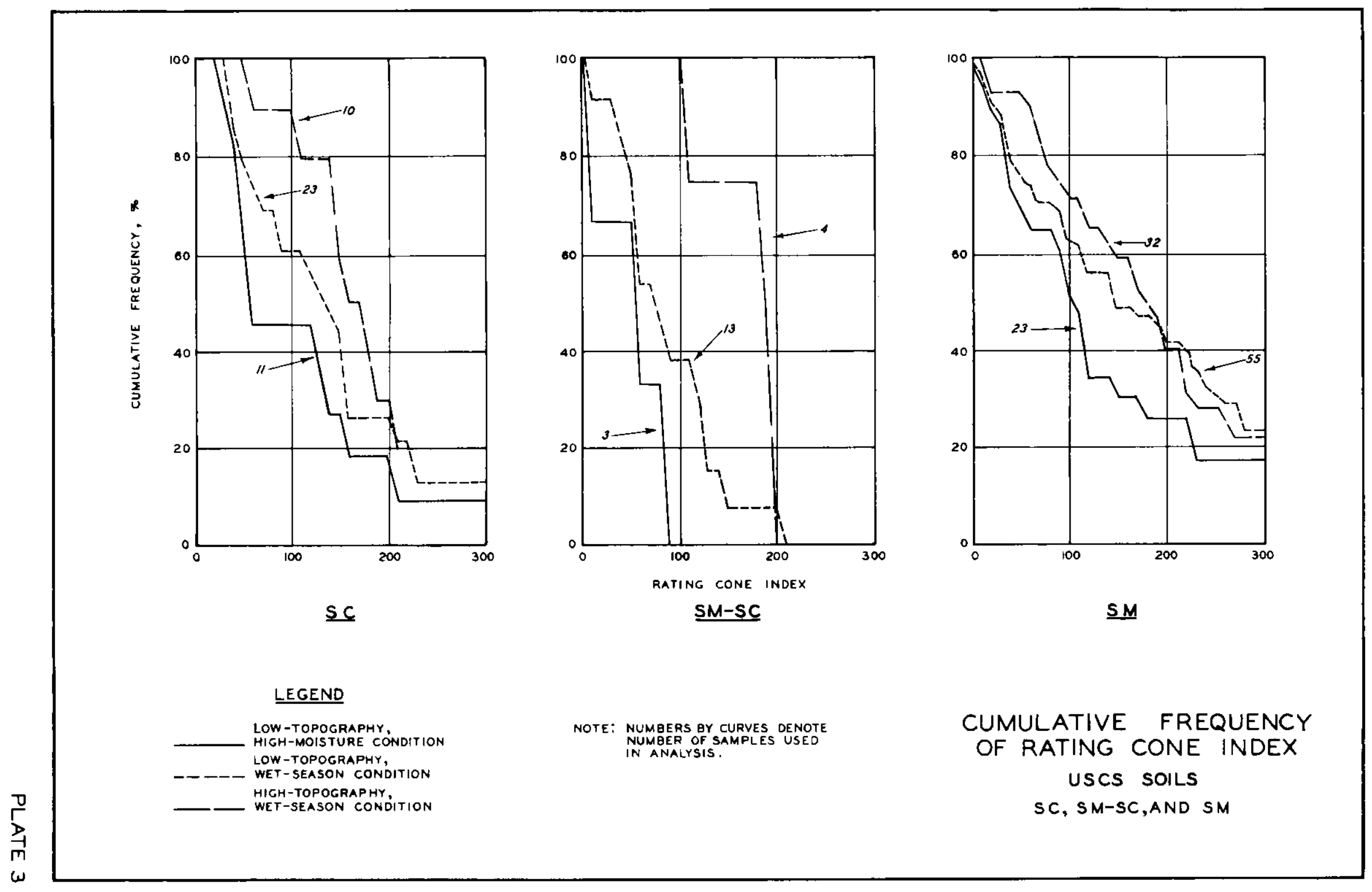




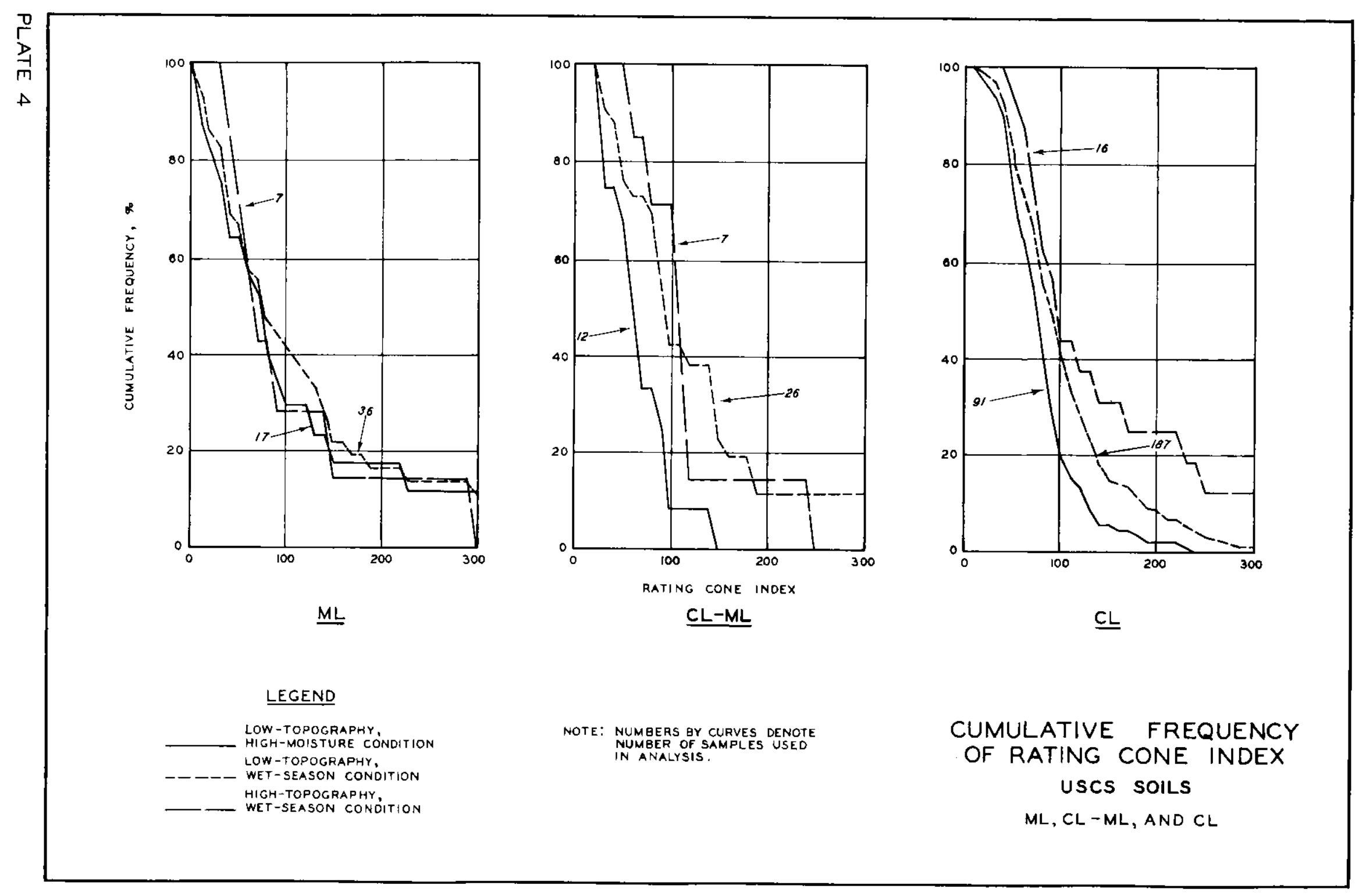




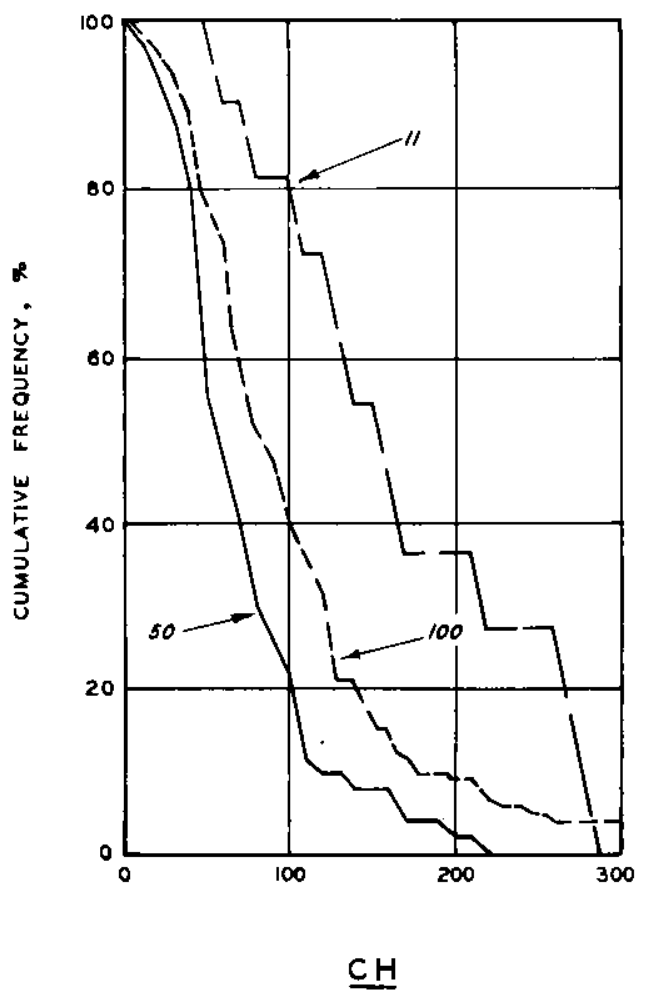

\section{LEGEND}

LOW-TOPOGRAPHY, HIGH-MOISTURE CONDITION

WET-SEASON CONDITION

HIGH-TOPOGRAPHY,
WET-SEASON CONDITION

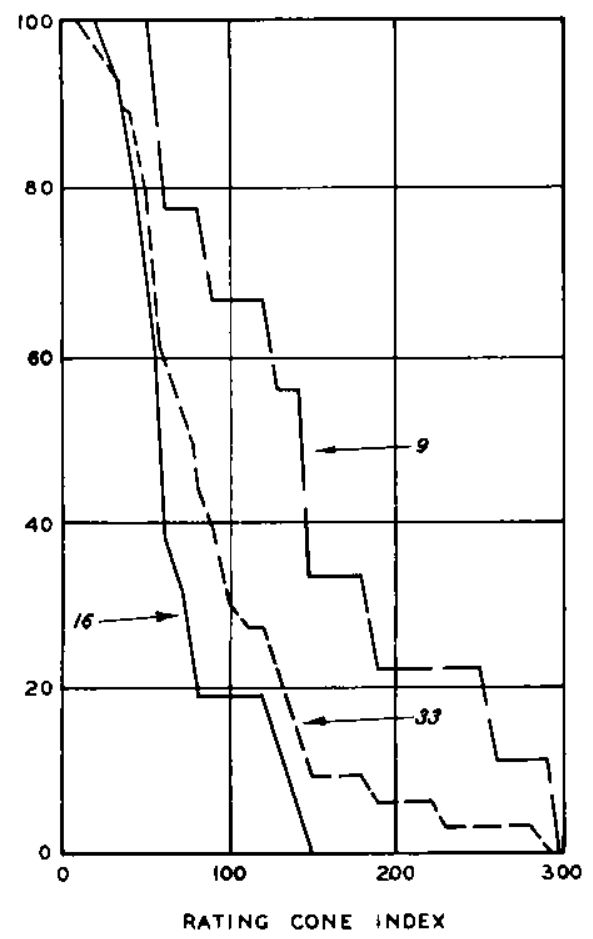

$\underline{\mathrm{MH}}$

NOTE: NUMEERS BY CURVES DENOTE NUMAER OF SAMPLES USEO IN ANALYSIS

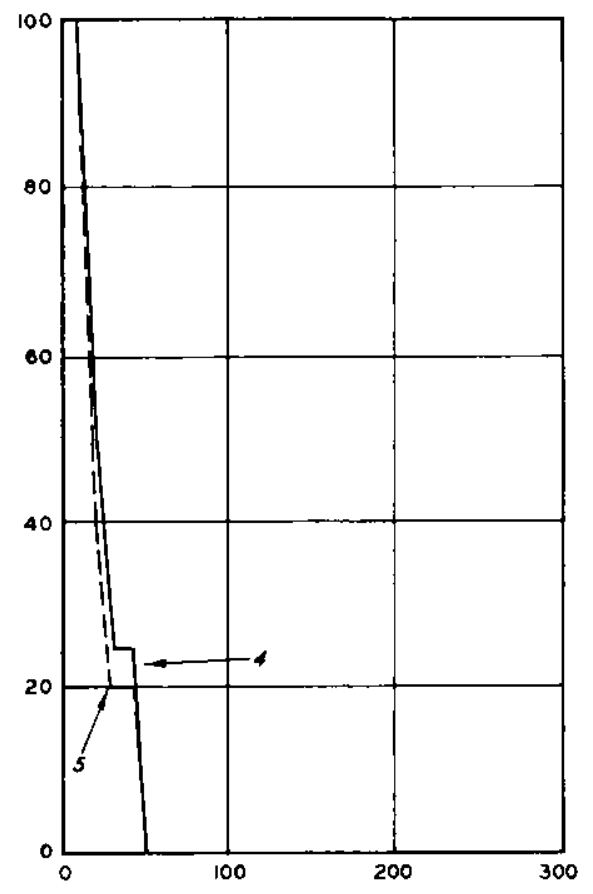

$\underline{\mathrm{OH}}$

CUMULATIVE FREQUENCY OF RATING CONE INDEX

USCS SOILS $\mathrm{CH}, \mathrm{MH}, \mathrm{AND} \mathrm{OH}$ 


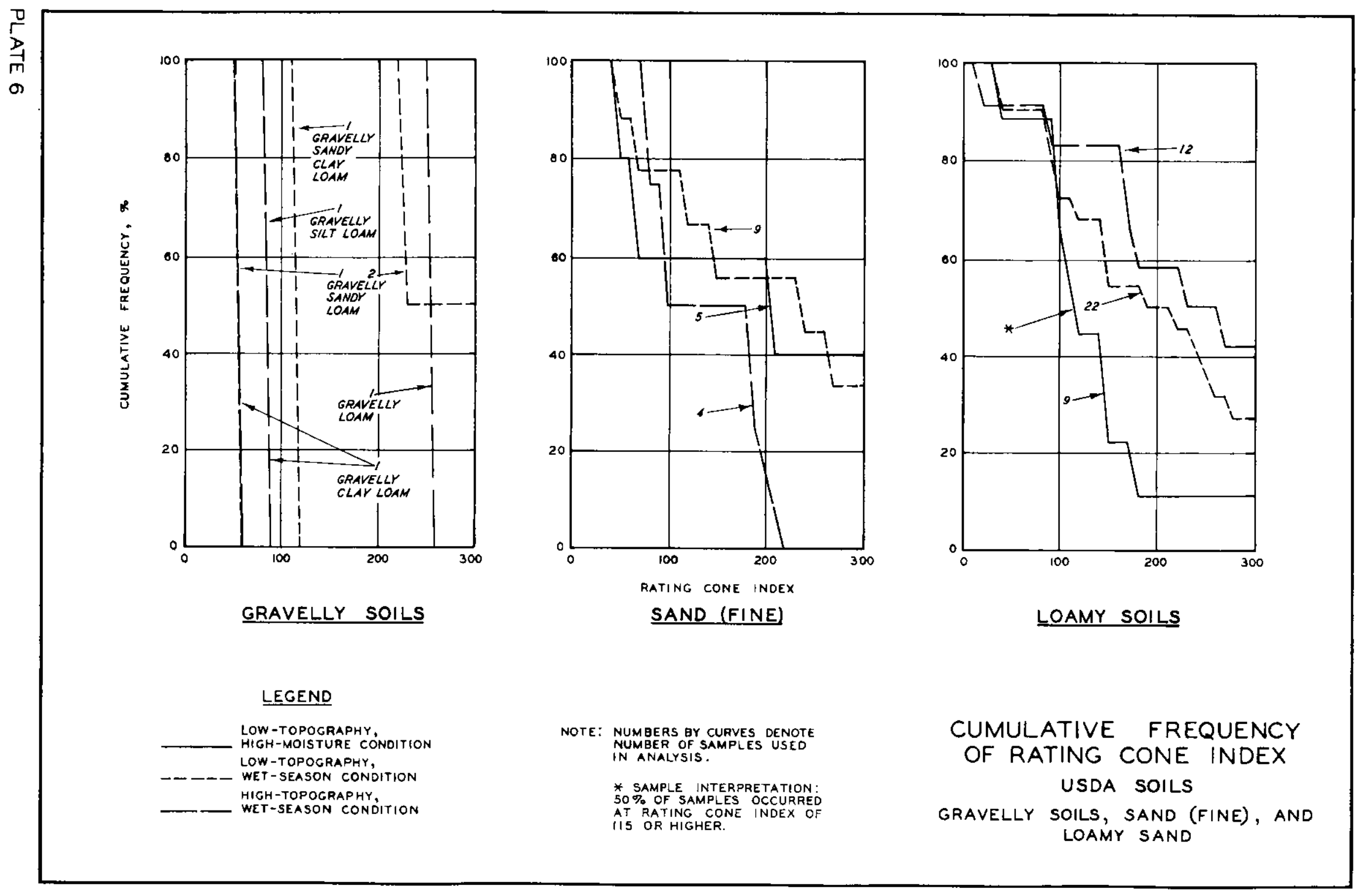




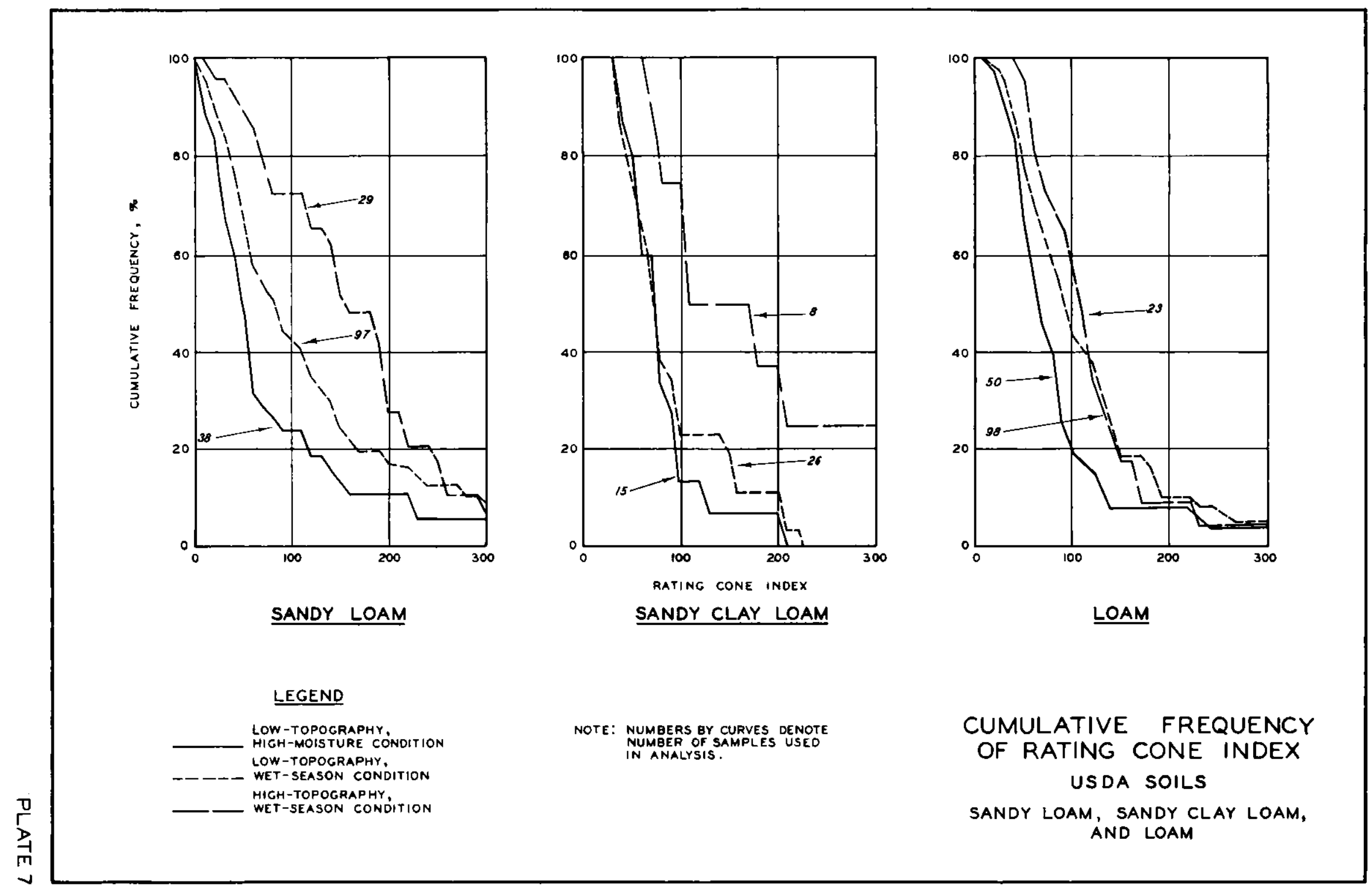




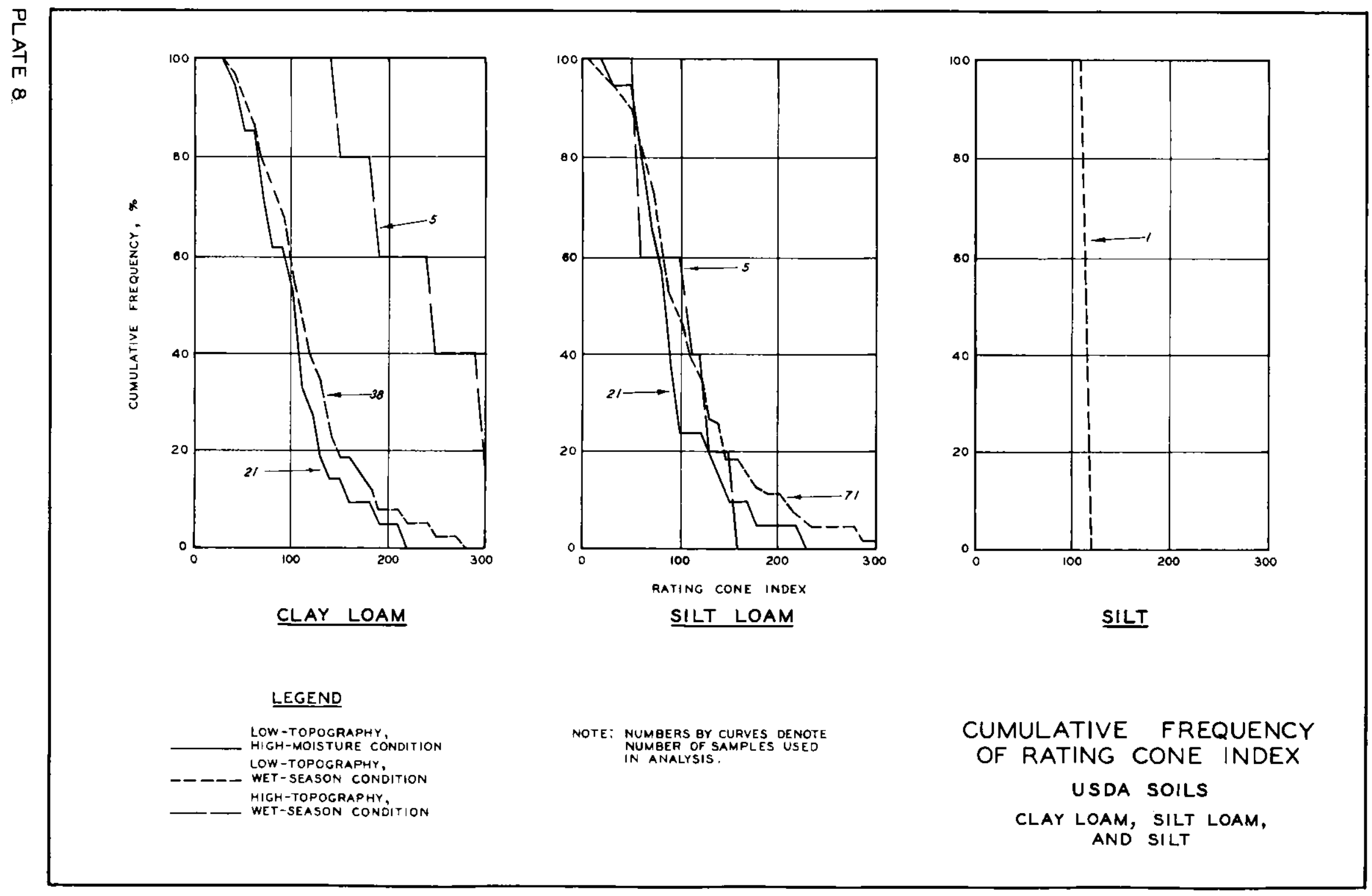




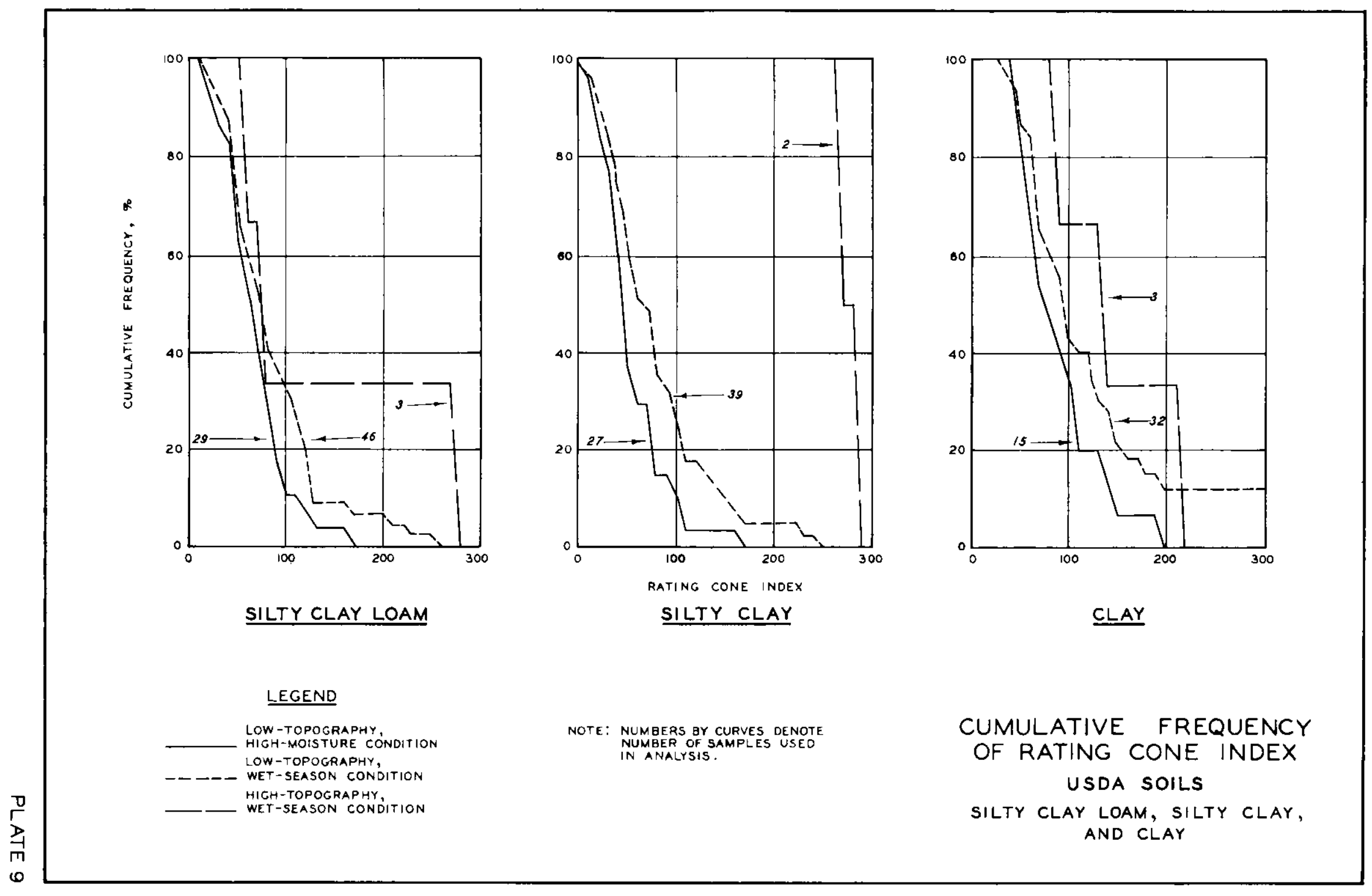




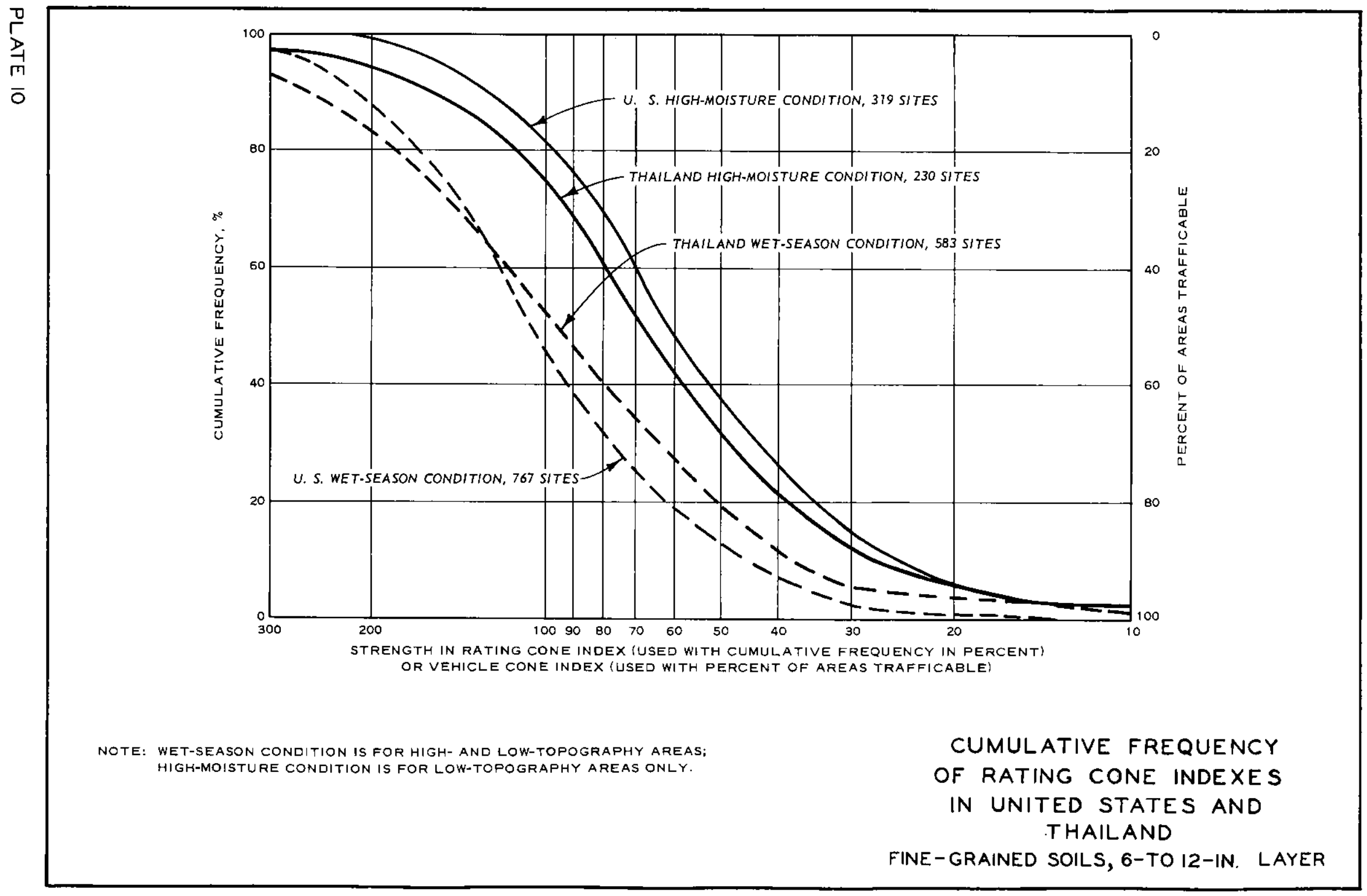


1. The soil and site data pertinent to the soil trafficability classification analyses are presented in tables Al-A6. These data were obtained in six different programs conducted during the period June 1962 through November 1965 in Thailand. Soil data included are texture, Atterberg limits, USCS and USDA soil type, organic content, and specific gravity. Trafficability data included are moisture content, density, depth to water table, and strength, i.e. cone index ( $\mathrm{CI}$ ), remolding index (RI), rating cone index (RCI), and sheargraph cohesion, adhesion and tan $\varnothing$ values, for wet-season and high-moisture conditions. Site data included are geographic locations furnished on Army Map Service map sheets and military grid coordinates, topographic class, topographic position, slope, vegetation, and land use. The general locations of the sites are shown on a map of Thailand in fig. 4 of the main text.

2. In tables Al-A6, the trafficability data for the wet-season condition are the data obtained during one visit to a site or an average of data for two or more visits during the wet season, as noted in the tables. At some sites the RI and, consequently, the RCI could not be determined on some visits because of the firmness of the soil. In determining an average RCI for a site which was visited two or more times, the CI data used were those for which RI measurements were available. Data for highmoisture conditions (water table 18 in. or less from the surface) for all trafficability parameters except sheargraph are usually for one visit. If this condition occurred on more than one visit, the data presented in the tables and used in the analyses were for the day of lowest RCI. Sheargraph data are listed under the high-moisture condition only when the water table was at the surface or free water was above the surface.

3. The following paragraphs are grouped according to the six sources of data and contain a detailed discussion of sampling techniques, number of visits to a site and number of measurements taken during each visit, number and geographical locations of the sites, and other important features of the test programs relevant to the data for each of the sources. 


\section{Preliminary Survey Study}

4. A preliminary study ${ }^{2}$ was made in Thailand to provide guidance for a planned, long-range research program to develop new methods and apply existing methods for measuring and predicting in quantitative and semiquantitative terms the effects of environmental factors on ground vehicles operating in Southeast Asia. Data were obtained from 202 sites visited during the period June-October 1962 by a team of specialist engineers, physical scientists, and technicians. The test sites were concentrated primarily in four geographic areas: the lower Chao Phraya Delta, the Bangkok Plain, the Khorat Plateau in south-central and eastern Thailand, and the Chiang Mai Basin in northwest Thailand. Also, some of the sites visited were located in the southeastern coastal plain. One visit was made to each site. The data collected for the preliminary survey study from 165 sites that are pertinent to this trafficability classification study are presented in table $\mathrm{Al}$.

Soil and trafficability data

5. At each test site, $10 \mathrm{CI}$ penetrations were made in an area approximately 10 by $20 \mathrm{ft}$. For each penetration, CI was measured at the surface and at 3 -in. vertical increments to a depth of $18 \mathrm{in.}$, and then at 6-in. vertical increments to a depth of $30 \mathrm{in.}$, when possible. When soil conditions permitted testing, RI was measured at each site for the $0-$ to 6 - and 6- to 12-in. layers. In some instances remolding index was measured for the 12- to 18-in. layer. Soil samples were taken for moisture contentdensity determinations for the same soil layers mentioned above. Representative bulk samples were taken from the 0- to 6- and 6- to 12-in. layers and occasionally from the 12- to I8-in. layer for laboratory tests. Samples were taken in an area approximately 1 ft square.

Site data

6. The site data obtained included geographic location of the test site, topographic position, slope, land use, vegetation, depth of soil, surface-water depth, and depth to water table. An attempt was made to sample a wide range of conditions and soil types with emphasis on testing the lowest and wettest areas on the assumption that the test results would 
give some insight into the maximum moisture contents and minimum strengths that various soil types would exhibit during the peak of the wet season.

\section{Trafficability Classification study}

7. Data collected for the trafficability classification study were obtained from 246 sites visited during the period August-October 1964 by a WES soil-trafficability team whose objective was to provide data specifically for the study reported herein. The sites were located within six MERS study areas: Nakhon Sawan, Lop Buri, Chiang Mai, Khon Kaen, Pran Buri, and Chanthaburi. In general, the rainy season extends from May to September in the northern area, from May to October in the central area, and from May to November in the south-central area of Thailand; hence, the period of testing coincided with the expected period of high soil-moisture content during the wettest monsoon season. One visit was made to each site. The data from 238 sites are presented in table A2. Soil and trafficability data

8. The strength data at each site were obtained as follows. Four sets of CI profiles were taken with measurements taken at the surface and at 3-in. vertical increments to a depth of 18 in.; RI tests were run on two or occasionally three samples from the 6- to 12-in. layer, and surface sheargraph measurements were made at three different locations on the site. Soil samples were taken from the 0- to 6- and 6- to 12-in. layers with the trafficability sampler for determination of moisture content, density, grain-size distribution, Atterberg limits, organic content, and specific gravity. When the soil was too firm for sampling with the trafficability sampler, samples were secured with an Oakfield punch or with a shovel. The locations of test or sampling points for each site are shown in fig. Al. Site data

9. In each area sites were selected to include a range of different soil types, topographic positions, land uses, and vegetation types with emphasis on the collection of data for soil type-topographic positions for which little or no data had previously been collected, e.g. moderately and highly organic soils on all topographic positions, all soils on upland 

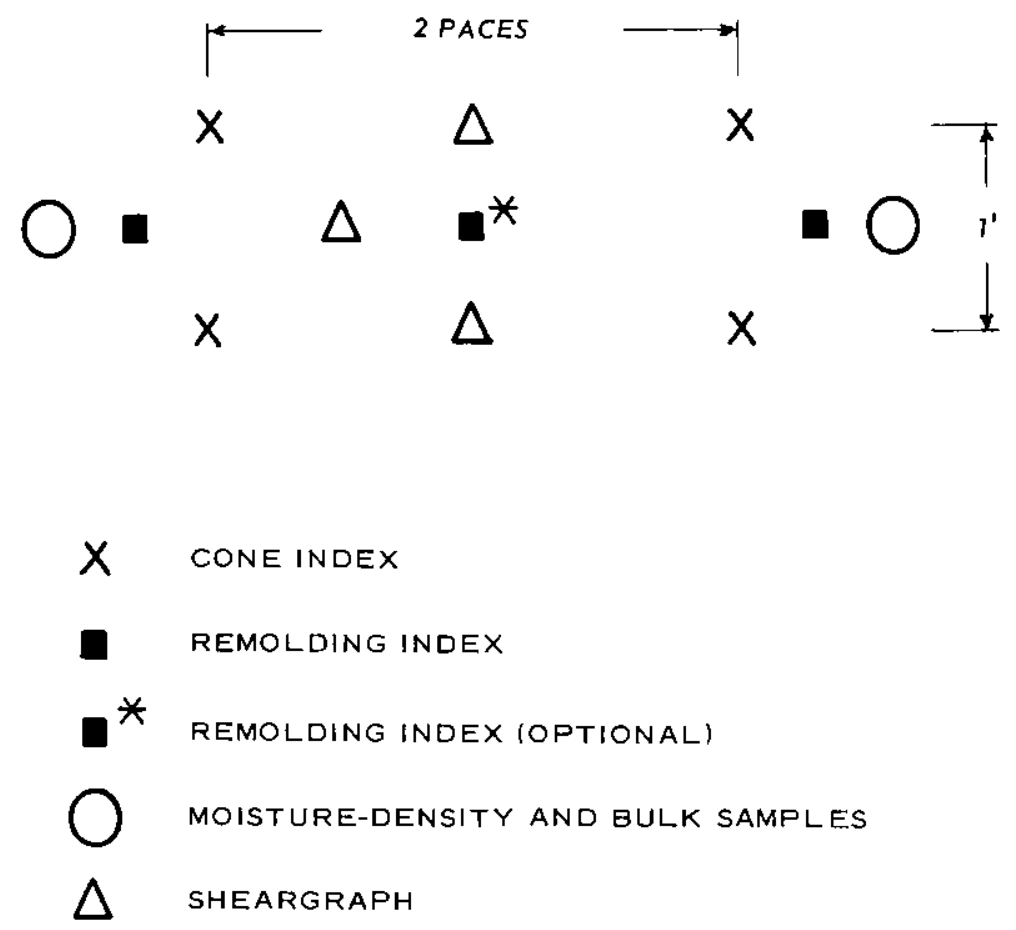

Fig. Al. Site layout

depressions and ridges, and clayey sands, silts, and clays on upland flats. A special effort was made to include an equal number of sites in each combination of soil type and topographic position.

10. Land use at each site was described in one or more of the following terms:

a. Undisturbed; not obviously used by man or domestic animals.

b. Disturbed; obviously used by man or domestic animals.

(I) Cropland currently in use (excluding hayfields, orchards, vineyards, tree plantations). Type

(2) Cropland currently lying fallow (excluding hayfields, orchards, vineyards, tree plantations). Type

(3) Pasture grazed by domestic animals.

(4) Hayfields (not currently being grazed).

(5) Orchards, vineyards, tree plantations. Type

(6) Lawns, recreation areas.

(7) Logged, cut for fuel, newly cleared for slash-and-burn agriculture. 
11. Since the essence of this program lay in securing a wide range of soil types and topographic position data during the high-moisture period of the wettest monsoon season, no attempt was made to collect detailed vegetation data; instead, the vegetation was described in general but consistent terms that could be readily identified even by relatively untrained observers. These terms are:

a. Forest. Trees more than $5 \mathrm{~m}$ (approximately $16 \mathrm{ft}$ ) tall with the crowns of the trees covering more than $90 \%$ of the area. Only the trees are significant; smaller plants are ignored.

b. Woodland. Trees more than $5 \mathrm{~m}$ (approximately $16 \mathrm{ft}$ ) tall with the crowns of the trees covering between 40 and $90 \%$ of the area.

c. Savanna. Trees more than $5 \mathrm{~m}$ (approximately $16 \mathrm{ft}$ ) tall with the crowns of the trees covering from 5 to $40 \%$ of the area. The "ground cover" may be wild grass, rice, maize, or any mostly herbaceous plants.

d. Tall scrub forest. Trees from 1.8 (approximately $6 \mathrm{ft}$ ) to $5 \mathrm{~m}$ (approximately $16 \mathrm{ft}$ ) tall with the crowns covering more than $90 \%$ of the area.

e. Tall scrub woodland. Trees from 1.8 (approximately $6 \mathrm{ft}$ ) to $5 \mathrm{~m}$ (approximately $16 \mathrm{ft}$ ) tall with the crowns covering 40 to $90 \%$ of the area.

f. Tall scrub savanna. Trees from 1.8 (approximately $6 \mathrm{ft}$ ) to $5 \mathrm{~m}$ (approximately $16 \mathrm{ft}$ ) tall with crowns covering from 5 to $40 \%$ of the area.

g. Low scrub. Shrubs from 0.7 (approximately $2.3 \mathrm{ft}$ ) to $1.8 \mathrm{~m}$ (approximately $6 \mathrm{ft}$ ) tall with the crowns covering more than $40 \%$ of the area.

h. Low scrub savanna. Shrubs from 0.7 (approximately $2.3 \mathrm{ft}$ ) to $1.8 \mathrm{~m}$ (approximately $6 \mathrm{ft}$ ) tall with the crowns covering from 5 to $40 \%$ of the area.

i. Tall-grass prairie. Herbaceous plants, usually of mostly grasses or grasslike plants, more than $0.7 \mathrm{~m}$ (approximately $2.3 \mathrm{ft}$ ) high with the plants covering more than $50 \%$ of the ground surface.

j. Short-grass prairie. Herbaceous plants, usually of mostly grasses or grasslike plants, less than $0.7 \mathrm{~m}$ (approximately $2.3 \mathrm{ft}$ ) high with the plants covering more than $50 \%$ of the ground surface.

k- Barren. More than $50 \%$ of the ground surface is bare, i.e. not covered by plants.

In the definitions listed above no distinction is made between cultivated 
and noncultivated plants. Thus, a coconut plantation, a rubber grove, or an orchard is usually a "forest" or a "woodland" and a field of mature rice is a "tall-grass prairie."

12. Topographic slope was measured with an Abney hand level at each site on the contiguous area over which no change in true slope occurred. Topographic position was identified as one of the following:

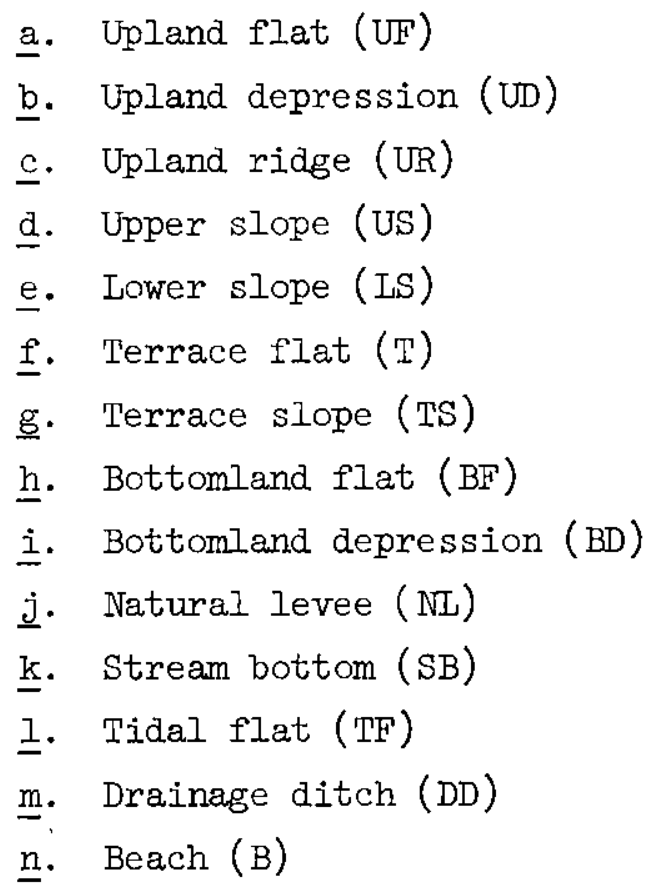

other data collected included depth of water over soil surface, depth to ground water, and depth to bedrock if within several feet of the surface.

\section{Surface Composition Study}

13. The objective of the surface composition study ${ }^{3}$ was to secure data for establishing the range in variation of areal and seasonal soil. strength in Thailand and for mapping soils exhibiting similar trafficability characteristics in selected study areas in Thailand. These data were obtained during the period April 1964 through June 1965 by a soil trafficability team. The study areas and the number of visits to sites in each area are shown in the following tabulation. 


\begin{tabular}{|c|c|}
\hline Area & $\begin{array}{l}\text { No. of Visits } \\
\text { to Site }\end{array}$ \\
\hline Nakhon Sawan & 2 \\
\hline Lop Buri & 3 \\
\hline Chiang Mai & 2 \\
\hline Pran Buri & 1 \\
\hline Khon Kaen & 2 \\
\hline Chanthaburi & 1 \\
\hline
\end{tabular}

The data collected for the surface composition study from 224 sites that are pertinent to the trafficability classification study are presented in table A3.

Soil and trafficability data

14. At each site, soil-strength data were collected for CI, RI, and sheargraph tests. Four or more CI profiles were obtained. Measurements for each profile were made at l-in. vertical increments to a depth of $18 \mathrm{in.}$ RI tests were conducted on samples from the 0- to 6- and 6- to 12-in. layers. If the results of the tests on two samples from each layer were not in close agreement, a third RI test was made. Sheargraph tests were performed on the soil surface at only one point on the site. Soil samples were taken with the trafficability sampler in 3-in. increments to a depth of $12 \mathrm{in.} \mathrm{for} \mathrm{the} \mathrm{determination} \mathrm{of} \mathrm{moisture} \mathrm{content} \mathrm{and} \mathrm{density.} \mathrm{Bulk}$ samples were taken for laboratory determination of grain-size distribution, Atterberg limits, organic content, and specific gravity. At each site a pit was dug and the soil profile was described to a depth of 18 in. Data also were obtained on the color, $\mathrm{pH}$, and reaction to $\mathrm{HCl}$ of soil horizons. From these data the soil series were identified.

Site data

15. The classification of site data for this study was the same as that used in the trafficability classification study (see paragraphs 10-12 of this appendix).

\section{Soil Moisture-Strength Study}

16. The objective of the soil moisture-strength study ${ }^{4}$ was to develop means for quantitatively predicting soil-moisture contents and 
strength of the soil for use in predicting trafficability of the critical soil layer. Data for this study were obtained from 75 sites during the period May 1964-November 1965 by teams of Thai engineers and technicians. Data were collected during two wet seasons and one dry season. Sites were selected to provide a range in climate, topography, soil type, and land use. The sites were located in seven MERS study areas and in the vicinity of Bangkok, Thailand.

17. Two types of sites, prediction-development (PD) and survey (TS), were established. Data from PD sites were used to develop rainfall-soil moisture-strength relations, and data from the TS sites will be used to check the accuracy of soil moisture-strength predictions that were based. on the relations developed from PD site data. Twenty PD and 55 TS sites were established. The PD sites were visited daily to collect unit electrical resistivity data for use in measurement of soil-moisture content, and these sites and the TS sites also were visited one or more times each month to collect trafficability data. The data from this study that are pertinent to the soil trafficability classification study are presented in table $\mathrm{A}_{4}$.

Soil and trafficability data

18. Data from the PD and TS sites were collected in a 21 - by 36-ft sampling area divided into 3-ft-square plots. Samples and direct measurements of the soil were taken in three randomly selected plots during each sampling visit to the site.

19. Six CI penetrations, two in each of three randomly selected plots, were made on each visit, and measurements of CI were averaged for the 0- to 6- and 6- to 12-in. layers. RI tests were performed on samples of the 6-to 12-in. layer from each of the three plots. Data from the tests were averaged for each visit. The RCI for a specific visit was then tabulated in the usual manner. An attempt was made to obtain strength measurements concurrently with moisture measurements. CI and especially RI data could not be obtained as frequently as moisture data and at some sites could not be obtained at all during the ary season due to firmness of the soil. At some sites flooding prevented data collection for long periods during the wet season. 
20. For each visit sheargraph measurements on the soil surface were made at two spots on each of the three selected plots and averaged. For most sites these data were obtained on four visits during the period of testing.

21. Soil samples of the 3-in. soil layers from the surface to a depth of $18 \mathrm{in.} \mathrm{were} \mathrm{taken} \mathrm{with} \mathrm{the} \mathrm{trafficability} \mathrm{sampler} \mathrm{for} \mathrm{determina-}$ tion of moisture content. Samples were obtained from two spots in each of three plots. The moisture content data were averaged for each 3-in. layer and for the 0 - to 6- and 6-to 12-in. layers.

22. When a thin-walled piston type soil sampler could be used to obtain a relatively undisturbed sample of proper length, that sample was saved for moisture content and density determinations. Also, when the soil was moist, two 2-in. cores were taken with the San Dimas or drivetype sampler for determination of density. The density listed in the summary table for each site is the average of density values determined from tests on piston-type soil samples, or where no such samples were obtained, it is the average of the two density values determined from tests on San Dimas soil samples.

23. The USDA and USCS soil types for each site were determined from mechanical analysis and Atterberg limits of bulk samples taken from the 0- to 6- and 6- to 12-in. layers. The sample tested in the laboratory for each layer was a composite of three samples taken at each end and along a point on one side of the site. The bulk soil samples were also tested to determine the organic content and specific gravity of the soil layer. Site data

24. Measurements of rainfall, depth to ground water, and ambient temperature were made daily at each of the PD sites.

25. Data describing the topographic position, slope, land use, and vegetation at a site were collected from observations in the field during the period of study. The systems for classifying topographic position, land use, and slope are the same as those used in the trafficability classification study (see paragraphs 10-12 of this appendix). 
U. S. Army Cold Regions Research and Engineering

Laboratory (CRREL) Airphoto Pattern Study

26. The purpose of the CRREL airphoto pattern study ${ }^{5}$ was to develop a method for interpreting, classifying, and mapping terrain features of Thailand from airphotos in terms of their effect on ground mobility. Data were obtained from 191 sites during the period 4 september-18 October 1964 by a team of specialist engineers and physical scientists.

27. The sites were located in two MERS study areas selected for detailed study--Lop Buri and Chanthaburi. The sites were selected primarily on the ability of the analyst to recognize tone and texture on aerial photographs. One visit was made to each site. The data from 121 sites pertinent to the trafficability classification study reported herein are presented in table $A 5$.

Soil and trafficability data

28. Three or more CI penetrations were made at each site. For each penetration, CI generally was measured at l-in. increments from the surface to a depth of $18 \mathrm{in.}$. RI tests were made on samples from the 6 - to 12-in. layer. Samples for determination of moisture content and density were taken with the trafficability sampler in 3-in. vertical increments from the surface to a depth of $12 \mathrm{in}$. The data were averaged for the $0-$ to 6- and 6- to 12-in. layers. (The density values are questionable and are not listed in the table.) When the soil was too firm to be sampled. with the trafficability sampler, samples for moisture only were taken with the Oakfield punch. Bulk samples for determination of grain size, Atterberg limits, organic content, and specific gravity were taken from the 0 to 6- and 6- to 12-in. layers. The soil profile was described briefly in pedological terms.

Site data

29. The topography class, topographic position, and land use identification for each site were based on a general description or were interpreted from aerial or ground photographs of the site. 
30. The terrain-vehicle test program ${ }^{6}$ was conducted to verify a mathematical model of cross-country vehicle performance previously developed in the United States, by applying it to tropical terrains and modifying it as required. Data for the program were collected in traffic test courses during the period September through October 1965 by a team of engineers, physical scientists, and technicians. The test courses ranged from 10 to $20 \mathrm{ft}$ (hydrologic geometry, designated HG, test courses), to several hundred feet (surface geometry, designated SG, and multiple, designated $M$, test courses), to several thousand feet (cross-country, designated CC, test courses) in length. For purposes of this study, each of the HG, SG, and M test courses was designated as a site. Each of the CC test courses was subdivided into two or more short stretches, based on changes in soil type, topography, and land use. These stretches were also designated as sites; e.g, test course CC2 was subdivided into sites CC2-A, -B, and -C. The data used in this report are from 23 sites located in the MERS study area of Khon Kaen. Data from the vehicle test program pertinent to the trafficability classification study are presented in table $A 6$.

Soil and trafficability data

31. The data in the table for each site are average values for the total number of visits. On each visit 10 or more CI penetrations were made. For each penetration, CI was measured at the surface, at depths of $I$ in. and 3 in., and then at 3-in. vertical increments to 24 or 30 in. Several RI tests were made on samples from the 0- to 6- and 6- to 12-in. layers. Sheargraph measurements of the soil. surface were taken in one small area. One set of samples per visit was obtained from the 0- to $1 / 4-$ and $0-$ to l-in. soil layers for determination of moisture content, and another set of samples was obtained from the 0-to 6- and 6- to 12-in. layers for determination of moisture content and density. Bulk samples for determination of grain size, Atterberg limits, and specific gravity were obtained from the $0-$ to 6 - and 6- to 12-in. layers. 
32. Geographic location, topography class, topographic position, slope, land use, and vegetation data were obtained from general terrain information secured in the field. Again, these data, as tabulated, represent average conditions of the test area. 
Tables A.1-A6 
Table AL

Irelisinary Survey sludy

Surmary of Site, Soil, and T.upficability Data.

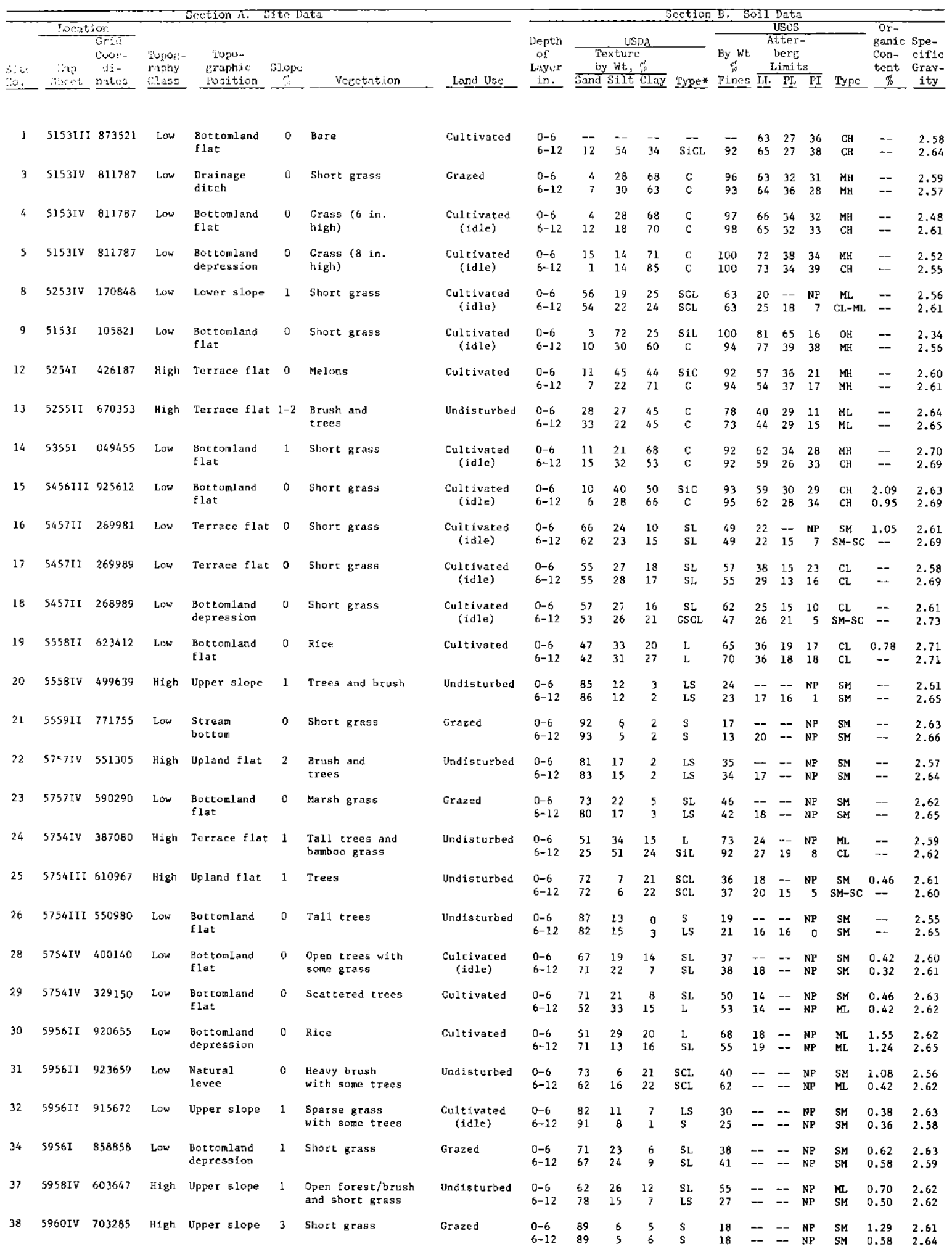

(Continued) 


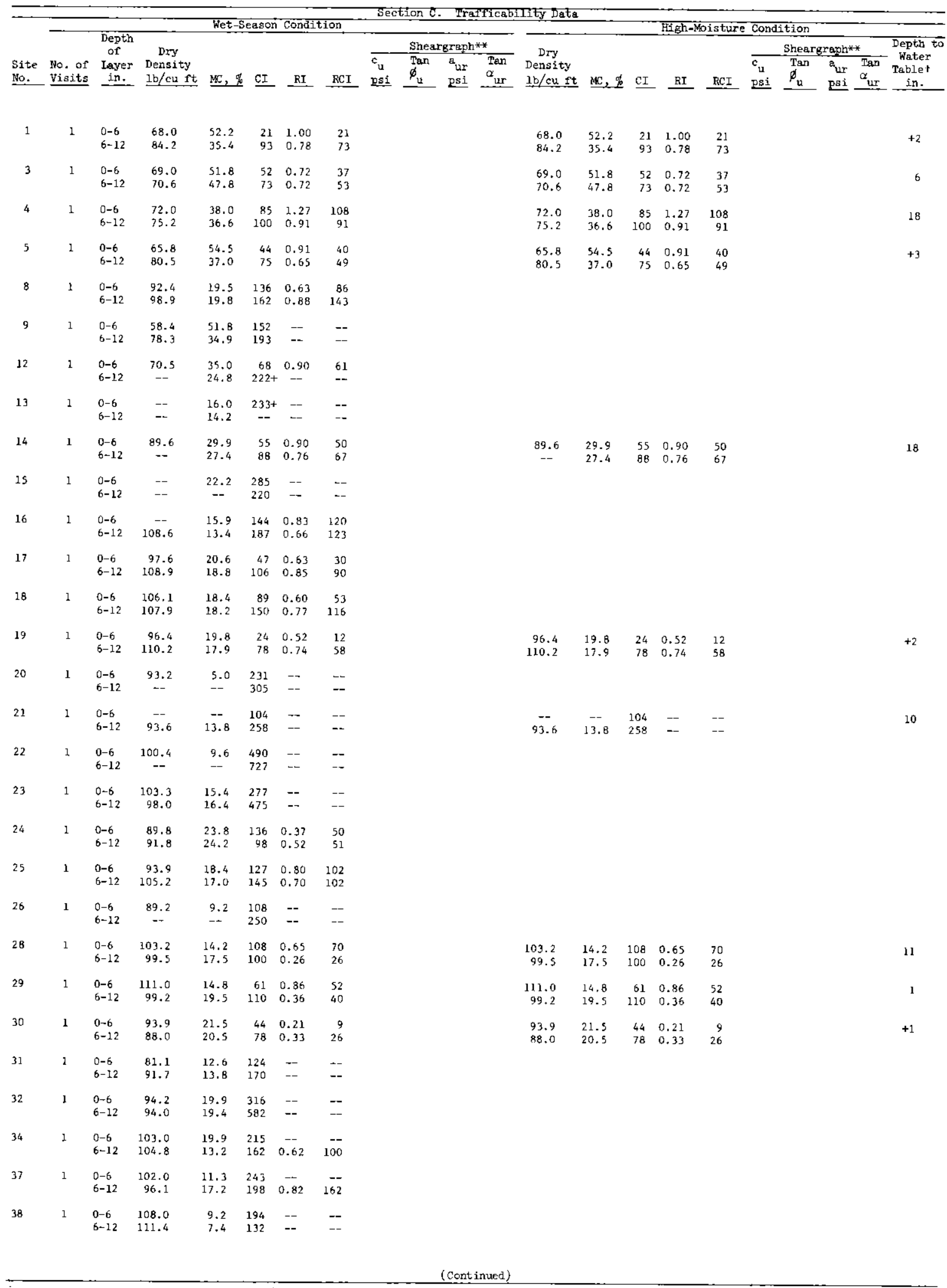

(Continued)

* $c_{u}$, ultimate soil-to-soil cohesion; $\phi_{u}$, ultimate soil-to-scil angle of internal friction; ar , wltimate soil-to-rubber adhesjon;

$\alpha_{u r}$, ultimate soil-to-rubber angle of friction.

+ Plus (+) denotes depth of water above surface.

( 2 of 12 sheets) 


\begin{tabular}{|c|c|c|c|c|c|c|c|c|c|c|c|c|c|c|c|c|c|c|c|}
\hline & & & & Stcction A. Si & itc Da & & 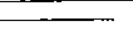 & & & & & Section & B. S & oil. & $\begin{array}{l}\text { Data } \\
\text { USCSS }\end{array}$ & & & or- & \\
\hline St t:- & $\begin{array}{c}\because x p \\
\text { suct } \\
\end{array}$ & $\begin{array}{l}\text { Grid } \\
\text { Luor- } \\
\text { di- } \\
\text { nates }\end{array}$ & $\begin{array}{l}\text { toposs- } \\
\text { raphy } \\
\text { class: } \\
\end{array}$ & $\begin{array}{l}\text { Lopo- } \\
\text { Lraphic } \\
\text { Posjtion }\end{array}$ & $\begin{array}{c}\text { Slope } \\
\text { in } \\
\text { in } \\
\end{array}$ & Veectition & Land Use & $\begin{array}{l}\text { Depth } \\
\text { of } \\
\text { Layer } \\
\text { in. } \\
\end{array}$ & $\begin{array}{r}-T \\
\quad b \\
\operatorname{sindi} \\
\end{array}$ & $\begin{array}{l}\text { us } \\
\text { exture } \\
y \text { wt, } \\
\text { sijt }\end{array}$ & $\begin{array}{l}\text { ISDA } \\
\text { re } \\
\frac{j}{t_{j}} \\
\text { Clisy }\end{array}$ & Sype & $\begin{array}{l}\text { By wt } \\
\text { Wis } \\
\text { lines } \\
\end{array}$ & & $\begin{array}{l}\text { teter } \\
\text { bere } \\
\text { Limits } \\
\text { PL }\end{array}$ & $\frac{s}{\text { PJ }}$ & Iype & $\begin{array}{l}\text { ganic: } \\
\text { Con- } \\
\text { tent } \\
0\end{array}$ & $\begin{array}{l}\text { Spe- } \\
\text { cific } \\
\text { Grav- } \\
\text { ity }\end{array}$ \\
\hline 39 & $59601 \mathrm{~V}$ & 700348 & Low & Terrace flat & 0 & Bare & Cultivated & $\begin{array}{l}0-6 \\
6-12\end{array}$ & $\begin{array}{l}51 \\
59\end{array}$ & $\begin{array}{l}36 \\
30\end{array}$ & $\begin{array}{l}13 \\
11\end{array}$ & $\begin{array}{l}\mathrm{L} \\
\mathrm{SL}\end{array}$ & $\begin{array}{l}65 \\
56\end{array}$ & $\begin{array}{l}-- \\
--\end{array}$ & $\begin{array}{l}-- \\
--\end{array}$ & $\begin{array}{l}\text { NP } \\
\text { NP }\end{array}$ & $\begin{array}{l}M L \\
M L\end{array}$ & $\begin{array}{l}0.74 \\
0.44\end{array}$ & $\begin{array}{l}2.63 \\
2.62\end{array}$ \\
\hline 40 & S960IV & 660420 & $\mathrm{High}$ & Upland flat & 0.5 & $\begin{array}{l}\text { Tall weeds with } \\
\text { scattered trees }\end{array}$ & $\begin{array}{l}\text { Cultivated } \\
\text { (idle) }\end{array}$ & $\begin{array}{l}0-6 \\
6-12\end{array}$ & $\begin{array}{l}68 \\
70\end{array}$ & $\begin{array}{l}11 \\
19\end{array}$ & $\begin{array}{l}21 \\
11\end{array}$ & $\begin{array}{l}\text { SCL } \\
\text { SL }\end{array}$ & $\begin{array}{l}45 \\
42\end{array}$ & -- & -- & $\begin{array}{l}\text { NP } \\
\text { NP }\end{array}$ & $\begin{array}{l}\text { SM } \\
\text { SM }\end{array}$ & $\begin{array}{l}1.46 \\
0.95\end{array}$ & $\begin{array}{l}2.61 \\
2.65\end{array}$ \\
\hline 41 & S960IV & 655415 & High & Lower slope & 1 & Trees & Undisturbed & $\begin{array}{l}0-6 \\
6-12\end{array}$ & $\begin{array}{l}82 \\
--\end{array}$ & $\begin{array}{l}18 \\
--\end{array}$ & $\begin{array}{c}0 \\
--\end{array}$ & $\begin{array}{l}\text { VGLS } \\
--\end{array}$ & $\begin{array}{l}13 \\
--\end{array}$ & $\begin{array}{l}-- \\
--\end{array}$ & $\begin{array}{l}-- \\
--\end{array}$ & $\begin{array}{l}\text { NP } \\
--\end{array}$ & $\begin{array}{l}\text { SM } \\
--\end{array}$ & $\begin{array}{c}1.15 \\
--\end{array}$ & $\begin{array}{c}2.76 \\
--\end{array}$ \\
\hline 42 & S962III & 698832 & Higi & Upper slope & 2 & Scattered trees & Undisturbed & $\begin{array}{l}0-6 \\
6-12\end{array}$ & $\begin{array}{l}72 \\
73\end{array}$ & $\begin{array}{l}12 \\
14\end{array}$ & $\begin{array}{l}16 \\
13\end{array}$ & $\begin{array}{l}\text { SL } \\
\text { GLS }\end{array}$ & $\begin{array}{l}31 \\
29\end{array}$ & $\begin{array}{l}-- \\
--\end{array}$ & $\begin{array}{l}-- \\
--\end{array}$ & $\begin{array}{l}\mathrm{NP} \\
\mathrm{NP}\end{array}$ & $\begin{array}{l}\text { SM } \\
\text { SM }\end{array}$ & $\begin{array}{l}0.90 \\
1.00\end{array}$ & $\begin{array}{l}2.64 \\
2.60\end{array}$ \\
\hline 43 & $\$ 963111$ & 614180 & High & Upland flat & 0 & Heavy tree growth & Undisturbed & $\begin{array}{l}0-6 \\
6-12\end{array}$ & $\begin{array}{l}50 \\
51\end{array}$ & $\begin{array}{l}29 \\
18\end{array}$ & $\begin{array}{l}21 \\
31\end{array}$ & $\begin{array}{c}\mathrm{GL} \\
V G S C L\end{array}$ & $\begin{array}{l}37 \\
23\end{array}$ & $\begin{array}{l}39 \\
52\end{array}$ & $\begin{array}{l}27 \\
32\end{array}$ & $\begin{array}{l}12 \\
20\end{array}$ & $\begin{array}{l}\text { SM } \\
\text { SM }\end{array}$ & $\begin{array}{l}2.20 \\
1.05\end{array}$ & $\begin{array}{l}3.14 \\
3.17\end{array}$ \\
\hline 44 & 5963111 & 590171 & Low & $\begin{array}{l}\text { Bottomland } \\
\text { f lat }\end{array}$ & 0 & $\begin{array}{l}\text { Low brush and } \\
\text { scattered trees }\end{array}$ & $\begin{array}{l}\text { Cultivated } \\
\quad \text { (idle) }\end{array}$ & $\begin{array}{l}0-6 \\
6-12\end{array}$ & $\begin{array}{l}35 \\
20\end{array}$ & $\begin{array}{l}46 \\
47\end{array}$ & $\begin{array}{l}19 \\
33\end{array}$ & $\stackrel{\mathrm{L}}{\mathrm{SiCl}}$ & $\begin{array}{l}81 \\
85\end{array}$ & $\begin{array}{l}38 \\
48\end{array}$ & $\begin{array}{l}26 \\
24\end{array}$ & $\begin{array}{l}12 \\
24\end{array}$ & $\begin{array}{l}\mathrm{ML}, \\
\mathrm{CL}\end{array}$ & $\begin{array}{l}2.87 \\
0.96\end{array}$ & $\begin{array}{l}2.64 \\
2.69\end{array}$ \\
\hline 45 & $\$ 862 I$ & 391151 & High & Upland $f$ lat & 0.5 & $\begin{array}{l}\text { Short grass and } \\
\text { scattered trees }\end{array}$ & $\begin{array}{l}\text { Cultivated } \\
\quad \text { (idle) }\end{array}$ & $\begin{array}{l}0-6 \\
6-12\end{array}$ & $\begin{array}{l}45 \\
39\end{array}$ & $\begin{array}{l}38 \\
28\end{array}$ & $\begin{array}{l}27 \\
33\end{array}$ & $\begin{array}{l}\mathrm{L} \\
\text { VGCL }\end{array}$ & $\begin{array}{l}91 \\
13\end{array}$ & $\begin{array}{l}16 \\
17\end{array}$ & $\begin{array}{l}-- \\
--\end{array}$ & $\begin{array}{l}\mathrm{NP} \\
\mathrm{NP}\end{array}$ & ${ }_{\text {ML }}^{M}$ & $\begin{array}{l}2.01 \\
1.29\end{array}$ & $\begin{array}{l}2.58 \\
2.91\end{array}$ \\
\hline 47 & S763III & 640201 & $\mathrm{High}$ & Upland flat & 0 & Trees & Undisturbed & $\begin{array}{l}0-6 \\
6-12\end{array}$ & $\begin{array}{l}69 \\
60\end{array}$ & $\begin{array}{l}24 \\
31\end{array}$ & $\begin{array}{l}7 \\
9\end{array}$ & $\begin{array}{l}\text { SL } \\
\text { SL }\end{array}$ & $\begin{array}{l}47 \\
47\end{array}$ & $\begin{array}{l}-- \\
--\end{array}$ & $\begin{array}{l}-- \\
--\end{array}$ & $\begin{array}{l}\mathrm{NP} \\
\mathrm{NP}\end{array}$ & $\begin{array}{l}S M \\
S M\end{array}$ & $\begin{array}{l}0.38 \\
0.25\end{array}$ & $\begin{array}{l}2.53 \\
2.53\end{array}$ \\
\hline 49 & 5763 III & 640220 & Low & Terrace flat & 0 & Rice & Cultivated & $\begin{array}{l}0-6 \\
6-12\end{array}$ & $\begin{array}{l}54 \\
56\end{array}$ & $\begin{array}{l}37 \\
41\end{array}$ & $\begin{array}{l}9 \\
3\end{array}$ & $\begin{array}{l}\text { SL } \\
\text { SL }\end{array}$ & $\begin{array}{l}60 \\
70\end{array}$ & $\overline{17}$ & $\overline{17}$ & $\begin{array}{r}\mathrm{NP} \\
\mathrm{O}\end{array}$ & $\begin{array}{l}\text { ML } \\
\text { ML }\end{array}$ & $\begin{array}{l}0.50 \\
0.44\end{array}$ & $\begin{array}{l}2.61 \\
2.63\end{array}$ \\
\hline 50 & S763III & 505279 & Low & Terrace flat & 0 & Bare & Cultivated & $\begin{array}{l}0-6 \\
6-12\end{array}$ & $\begin{array}{l}46 \\
25\end{array}$ & $\begin{array}{l}32 \\
24\end{array}$ & $\begin{array}{l}22 \\
51\end{array}$ & $\begin{array}{l}\text { L } \\
\mathrm{C}\end{array}$ & $\begin{array}{l}85 \\
90\end{array}$ & $\begin{array}{l}17 \\
35\end{array}$ & $\overline{21}$ & $\begin{array}{l}\text { NP } \\
14\end{array}$ & $\begin{array}{l}\mathrm{ML} \\
\mathrm{CL}\end{array}$ & $\begin{array}{l}0.58 \\
0.62\end{array}$ & $\begin{array}{l}2.55 \\
2.55\end{array}$ \\
\hline 51 & 556311 & 683239 & Low & Terrace flat & 0 & Short grass & $\begin{array}{l}\text { Cultivated } \\
\quad \text { (idle) }\end{array}$ & $\begin{array}{l}0-6 \\
6-12\end{array}$ & $\begin{array}{l}72 \\
43\end{array}$ & $\begin{array}{l}20 \\
28\end{array}$ & $\begin{array}{r}8 \\
29\end{array}$ & $\begin{array}{l}\text { SL } \\
\text { CL }\end{array}$ & $\begin{array}{l}53 \\
62\end{array}$ & $\overline{17}$ & $\begin{array}{l}-- \\
--\end{array}$ & $\begin{array}{l}\text { NP } \\
\text { NP }\end{array}$ & $\begin{array}{l}\text { ML. } \\
\text { ML. }\end{array}$ & $\begin{array}{l}0.46 \\
0.39\end{array}$ & $\begin{array}{l}2.58 \\
2.58\end{array}$ \\
\hline 52 & 54621 & 312063 & High & Lower slope & 15 & $\begin{array}{l}\text { Scattered trees } \\
\text { and till grass }\end{array}$ & Undisturbed & $\begin{array}{l}0-6 \\
6-12\end{array}$ & $\begin{array}{l}82 \\
60\end{array}$ & $\begin{array}{r}8 \\
26\end{array}$ & $\begin{array}{l}10 \\
14\end{array}$ & $\begin{array}{l}\text { LS } \\
\text { SL }\end{array}$ & $\begin{array}{l}50 \\
\text { so }\end{array}$ & $\overline{22}$ & $\overline{17}$ & $\begin{array}{r}\mathrm{NP} \\
5\end{array}$ & $\begin{array}{c}S M \\
S M-S C\end{array}$ & $\begin{array}{l}0.86 \\
0.55\end{array}$ & $\begin{array}{l}2.53 \\
2.68\end{array}$ \\
\hline 53 & $5462 I$ & 326070 & High & Lower slope & 7 & $\begin{array}{l}\text { Bamboo with } \\
\text { scattered trees }\end{array}$ & Undisturbed & $\begin{array}{l}0-6 \\
6-12\end{array}$ & $\begin{array}{l}45 \\
46\end{array}$ & $\begin{array}{l}19 \\
16\end{array}$ & $\begin{array}{l}36 \\
38\end{array}$ & $\begin{array}{l}\mathrm{CL} \\
\mathrm{SC}\end{array}$ & $\begin{array}{l}55 \\
55\end{array}$ & $\begin{array}{l}22 \\
23\end{array}$ & $\begin{array}{l}-- \\
--\end{array}$ & $\begin{array}{l}\text { NP } \\
\text { NP }\end{array}$ & $\begin{array}{l}\text { ML } \\
\text { ML }\end{array}$ & $\begin{array}{l}1.55 \\
1.15\end{array}$ & $=$ \\
\hline 54 & $5563 I I$ & 683239 & Low & Terrace flat & 0 & Short grass & $\begin{array}{l}\text { Cultivated } \\
\quad \text { (idle) }\end{array}$ & $\begin{array}{l}0-6 \\
6-12\end{array}$ & $\begin{array}{l}72 \\
44\end{array}$ & $\begin{array}{l}20 \\
28\end{array}$ & $\begin{array}{r}8 \\
30\end{array}$ & $\begin{array}{l}\mathrm{SL} \\
\mathrm{CL}\end{array}$ & $\begin{array}{l}53 \\
62\end{array}$ & $\overline{17}$ & $\begin{array}{l}-- \\
--\end{array}$ & $\begin{array}{l}\text { NP } \\
\text { NP }\end{array}$ & $\begin{array}{l}M L \\
M L\end{array}$ & $\begin{array}{l}0.46 \\
0.39\end{array}$ & $\begin{array}{l}-- \\
--\end{array}$ \\
\hline 55 & 556111 & 661493 & High & Upland flat & 1 & Trees and brush & Undisturbed & $\begin{array}{l}0-6 \\
6-12\end{array}$ & $\begin{array}{l}94 \\
82\end{array}$ & $\begin{array}{r}6 \\
14\end{array}$ & $\begin{array}{l}0 \\
4\end{array}$ & $\begin{array}{l}\text { S } \\
\text { LS }\end{array}$ & $\begin{array}{l}28 \\
30\end{array}$ & -- & -- & $\begin{array}{r}\text { NP } \\
1\end{array}$ & $\begin{array}{l}S M \\
S M\end{array}$ & $\begin{array}{l}0.32 \\
0.25\end{array}$ & $\begin{array}{l}2.62 \\
2.62\end{array}$ \\
\hline 56 & SS60II & 696228 & Bigh & Upper slope & 1 & Brush and trees & Und fsturbed & $\begin{array}{l}0-6 \\
6-12\end{array}$ & $\begin{array}{l}87 \\
71\end{array}$ & $\begin{array}{l}13 \\
22\end{array}$ & 0 & $\begin{array}{l}\text { S } \\
\text { SL. }\end{array}$ & $\begin{array}{l}35 \\
38\end{array}$ & $\overline{13}$ & $\overline{13}$ & $\begin{array}{r}\text { NP } \\
0\end{array}$ & $\begin{array}{l}S M \\
S M\end{array}$ & $\begin{array}{l}0.74 \\
0.62\end{array}$ & $\begin{array}{l}2.59 \\
2.66\end{array}$ \\
\hline 57 & $54581 I$ & 142367 & High & Upper slope & 3 & Short grass & $\begin{array}{l}\text { Cultivated } \\
\text { (idle) }\end{array}$ & $\begin{array}{l}0-6 \\
6-12\end{array}$ & $\begin{array}{l}82 \\
80\end{array}$ & $\begin{array}{l}14 \\
17\end{array}$ & $\begin{array}{l}4 \\
3\end{array}$ & $\begin{array}{l}\text { LS } \\
\text { LS }\end{array}$ & $\begin{array}{l}28 \\
28\end{array}$ & $\begin{array}{l}20 \\
20\end{array}$ & $\overline{18}$ & $\begin{array}{r}\mathrm{NP} \\
2\end{array}$ & $\begin{array}{l}S M \\
S M\end{array}$ & $\begin{array}{l}0.70 \\
0.42\end{array}$ & $\begin{array}{l}2.64 \\
2.62\end{array}$ \\
\hline 58 & 535811 & 204351 & Low & $\begin{array}{l}\text { Bottomland } \\
\text { flat }\end{array}$ & 0 & Bare & Cultivated & $\begin{array}{l}0-6 \\
6-12\end{array}$ & $\begin{array}{r}100 \\
74\end{array}$ & $\begin{array}{l}0 \\
9\end{array}$ & $\begin{array}{r}0 \\
17\end{array}$ & $\begin{array}{l}S \\
S L\end{array}$ & $\begin{array}{l}32 \\
42\end{array}$ & $\overline{22}$ & -- & $\begin{array}{l}\text { NP } \\
\text { NP }\end{array}$ & $\begin{array}{l}\text { SM } \\
\text { SM }\end{array}$ & $\begin{array}{l}1.77 \\
0.70\end{array}$ & $\begin{array}{l}2.61 \\
2.64\end{array}$ \\
\hline 59 & 535711 & 033062 & High & Upper slope & 1 & Short grass & Lawn & $\begin{array}{l}0-6 \\
6-12\end{array}$ & $\begin{array}{r}100 \\
71\end{array}$ & $\begin{array}{r}0 \\
17^{0}\end{array}$ & $\begin{array}{r}0 \\
12 .\end{array}$ & $\begin{array}{l}\mathrm{S} \\
\mathrm{SL}\end{array}$ & $\begin{array}{l}34 \\
38\end{array}$ & $\overline{18}$ & $\overrightarrow{--}$ & $\begin{array}{l}\mathrm{NP} \\
\mathrm{MP}\end{array}$ & $\begin{array}{l}\text { SM } \\
\text { SM }\end{array}$ & $\begin{array}{l}0.88 \\
0.70\end{array}$ & $\begin{array}{l}2.62 \\
2.62\end{array}$ \\
\hline 60 & 515611 & 001670 & Low & $\begin{array}{l}\text { Bottomland } \\
\text { flat }\end{array}$ & 0 & Rice & Cultivated & $\begin{array}{l}0-6 \\
6-12\end{array}$ & $\begin{array}{l}40 \\
32\end{array}$ & $\begin{array}{l}30 \\
45\end{array}$ & $\begin{array}{l}30 \\
23\end{array}$ & $\begin{array}{l}\text { c } \\
\text { C }\end{array}$ & $\begin{array}{l}66 \\
70\end{array}$ & $\begin{array}{l}50 \\
68\end{array}$ & $\begin{array}{l}19 \\
26\end{array}$ & $\begin{array}{l}31 \\
42\end{array}$ & $\begin{array}{l}\mathrm{CH} \\
\mathrm{CH}\end{array}$ & $\begin{array}{l}0.88 \\
0.46\end{array}$ & $\begin{array}{l}2.57 \\
2.56\end{array}$ \\
\hline 62 & S1511V & 770977 & Low & $\begin{array}{l}\text { Bottomland } \\
\text { flat }\end{array}$ & 0 & Short grass & $\begin{array}{l}\text { Cultivated } \\
\text { (1dle) }\end{array}$ & $\begin{array}{l}0-6 \\
6-12\end{array}$ & $\frac{9}{7}$ & $\begin{array}{l}37 \\
36\end{array}$ & $\begin{array}{l}54 \\
57\end{array}$ & $\begin{array}{l}\mathrm{C} \\
\mathrm{C}\end{array}$ & $\begin{array}{l}92 \\
95\end{array}$ & -- & $\overline{28}$ & -- & $\overline{C L}$ & $\begin{array}{l}0.38 \\
0.32\end{array}$ & $\begin{array}{l}-- \\
2.72\end{array}$ \\
\hline 63 & $5151 \mathrm{IV}$ & 770977 & Low & $\begin{array}{l}\text { Bottomland } \\
\text { flat }\end{array}$ & 0 & Short grass & $\begin{array}{l}\text { Cultivated } \\
\text { (idle) }\end{array}$ & $\begin{array}{l}0-6 \\
6-12\end{array}$ & $\begin{array}{l}6 \\
8\end{array}$ & $\begin{array}{l}36 \\
34\end{array}$ & $\begin{array}{l}58 \\
58\end{array}$ & $\begin{array}{l}\mathrm{c} \\
\mathrm{c}\end{array}$ & $\begin{array}{l}96 \\
93\end{array}$ & $\overline{72}$ & -- & $\begin{array}{l}-- \\
42\end{array}$ & $\overline{\mathrm{CH}}$ & $\begin{array}{l}0.32 \\
0.84\end{array}$ & 2.74 \\
\hline 66 & $\$ 1541 I$ & 061031 & Low & $\begin{array}{l}\text { Bottomland } \\
\text { flat }\end{array}$ & 0 & Short grass & $\begin{array}{l}\text { Cultivated } \\
\quad \text { (idle) }\end{array}$ & $\begin{array}{l}0-6 \\
6-12\end{array}$ & $\begin{array}{l}39 \\
42\end{array}$ & $\begin{array}{l}49 \\
45\end{array}$ & $\begin{array}{l}12 \\
13\end{array}$ & $\begin{array}{l}\mathrm{L} \\
\mathrm{L}\end{array}$ & $\begin{array}{l}67 \\
64\end{array}$ & $\overline{18}$ & $\overline{14}$ & -- & $\overline{C L}-\overline{M L}$ & $\begin{array}{l}0.58 \\
0.38\end{array}$ & $2 .-65$ \\
\hline 67 & $5151 \mathrm{IV}$ & 795940 & Low & $\begin{array}{l}\text { Bottomland } \\
\text { flat }\end{array}$ & 0 & Brush & Grazed & $\begin{array}{l}0-6 \\
6-12\end{array}$ & $\begin{array}{l}9 \\
6\end{array}$ & $\begin{array}{l}39 \\
47\end{array}$ & $\begin{array}{l}52 \\
47\end{array}$ & $\begin{array}{c}\mathrm{C} \\
\mathrm{Sic}\end{array}$ & $\begin{array}{l}97 \\
97\end{array}$ & $\overline{79}$ & $\overline{25}$ & $\overline{54}$ & $\overline{\mathrm{CH}}$ & $\begin{array}{l}1.82 \\
1.72\end{array}$ & 2.75 \\
\hline 68 & $5151 \mathrm{IV}$ & 795940 & Low & $\begin{array}{l}\text { Bottomland } \\
\text { flat }\end{array}$ & 0 & $\begin{array}{l}\text { Brush and } \\
\text { short grass }\end{array}$ & Grazed & $\begin{array}{l}0-6 \\
6-12\end{array}$ & $\begin{array}{r}12 \\
5\end{array}$ & $\begin{array}{l}43 \\
48\end{array}$ & $\begin{array}{l}45 \\
47\end{array}$ & $\begin{array}{l}\text { Sic } \\
\text { Sic }\end{array}$ & $\begin{array}{l}91 \\
97\end{array}$ & $\begin{array}{l}70 \\
69\end{array}$ & $\begin{array}{l}32 \\
30\end{array}$ & $\begin{array}{l}38 \\
39\end{array}$ & $\begin{array}{l}\mathrm{CH} \\
\mathrm{CH}\end{array}$ & $\begin{array}{l}2.22 \\
2.20\end{array}$ & $\overline{2.72}$ \\
\hline 69 & $5151 \mathrm{IV}$ & 795940 & Low & $\begin{array}{l}\text { Bottomland } \\
\text { flat }\end{array}$ & 0 & $\begin{array}{l}\text { Brush and } \\
\text { short grass }\end{array}$ & Grazed & $\begin{array}{l}0-6 \\
6-12\end{array}$ & $\begin{array}{l}5 \\
4\end{array}$ & $\begin{array}{l}56 \\
46\end{array}$ & $\begin{array}{l}39 \\
50\end{array}$ & $\begin{array}{l}\mathrm{SiCL} \\
\mathrm{SiC}\end{array}$ & $\begin{array}{l}97 \\
97\end{array}$ & $\begin{array}{l}86 \\
97\end{array}$ & $\begin{array}{l}34 \\
36\end{array}$ & $\begin{array}{l}52 \\
61\end{array}$ & $\begin{array}{l}\mathrm{CH} \\
\mathrm{CH}\end{array}$ & $\begin{array}{l}3.87 \\
3.58\end{array}$ & 2.73 \\
\hline 70 & $5150 \mathrm{II}$ & 087445 & High & Terrace flat & 0 & $\begin{array}{l}\text { Recently cleared } \\
\text { of trees }\end{array}$ & $\begin{array}{l}\text { Fruft } \\
\text { plantation }\end{array}$ & $\begin{array}{l}0-6 \\
6-12\end{array}$ & $\begin{array}{l}76 \\
75\end{array}$ & $\begin{array}{l}18 \\
17\end{array}$ & $\begin{array}{l}6 \\
8\end{array}$ & $\begin{array}{l}\text { SL } \\
\text { SL }\end{array}$ & $\begin{array}{l}28 \\
29\end{array}$ & $\overline{11}$ & -- & $\overline{\mathrm{NP}}$ & $\overline{S M}$ & $\begin{array}{l}-- \\
--\end{array}$ & -- \\
\hline 71 & 52481 & 530000 & Low & $\begin{array}{l}\text { Bot tom 1and } \\
\text { flat }\end{array}$ & 0 & Rice & Cultivated & $\begin{array}{l}0-6 \\
6-12\end{array}$ & $\begin{array}{l}73 \\
75\end{array}$ & $\begin{array}{l}9 \\
5\end{array}$ & $\begin{array}{l}18 \\
20\end{array}$ & $\begin{array}{l}\text { SL } \\
\text { SL }\end{array}$ & $\begin{array}{l}32 \\
27\end{array}$ & -- & $\overline{15}$ & $-\overline{8}$ & $\overline{\text { SC }}$ & $\begin{array}{l}1.35 \\
1.20\end{array}$ & $\overline{2.54}$ \\
\hline 73 & $S 2481$ & 635955 & Low & Beach & 0.5 & Bare & Undisturbed & $\begin{array}{l}0-6 \\
6-12\end{array}$ & $\begin{array}{l}96 \\
94\end{array}$ & $\begin{array}{l}2 \\
3\end{array}$ & $\begin{array}{l}2 \\
3\end{array}$ & $\begin{array}{l}\mathrm{s} \\
\mathrm{s}\end{array}$ & $\begin{array}{l}20 \\
20\end{array}$ & $\overline{27}$ & -- & $\overline{\mathrm{NP}}$ & $\overline{S M}$ & $\begin{array}{l}0.22 \\
0.25\end{array}$ & $2 . \overline{68}$ \\
\hline 74 & 5449111 & 973040 & Low & Terrace flat & 0 & Short grass & Grazed & $\begin{array}{l}0-6 \\
6-12\end{array}$ & $\begin{array}{l}45 \\
52\end{array}$ & $\begin{array}{l}52 \\
37\end{array}$ & $11^{3}$ & $\begin{array}{l}\text { SIL } \\
\text { SI }\end{array}$ & $\begin{array}{l}70 \\
58\end{array}$ & $\begin{array}{l}17 \\
14\end{array}$ & $\begin{array}{l}17 \\
14\end{array}$ & $\begin{array}{l}0 \\
0\end{array}$ & $\begin{array}{l}M L \\
M L\end{array}$ & $\begin{array}{l}2.91 \\
0.84\end{array}$ & $\overline{2.64}$ \\
\hline 75 & $\$ 449111$ & 945008 & High & Upper slope & 6 & $\begin{array}{l}\text { Rubber } \\
\text { plantation }\end{array}$ & Cultivated & $\begin{array}{l}0-6 \\
6-12\end{array}$ & $\begin{array}{l}64 \\
62\end{array}$ & $\begin{array}{l}19 \\
12\end{array}$ & $\begin{array}{l}17 \\
26\end{array}$ & $\begin{array}{l}\text { SL } \\
\text { SCL }\end{array}$ & $\begin{array}{l}40 \\
43\end{array}$ & $\begin{array}{l}34 \\
42\end{array}$ & $\begin{array}{l}24 \\
20\end{array}$ & $\begin{array}{l}10 \\
22\end{array}$ & $\begin{array}{l}\text { SM } \\
\text { SC }\end{array}$ & $\begin{array}{l}3.94 \\
2.49\end{array}$ & $\overline{2.64}$ \\
\hline
\end{tabular}




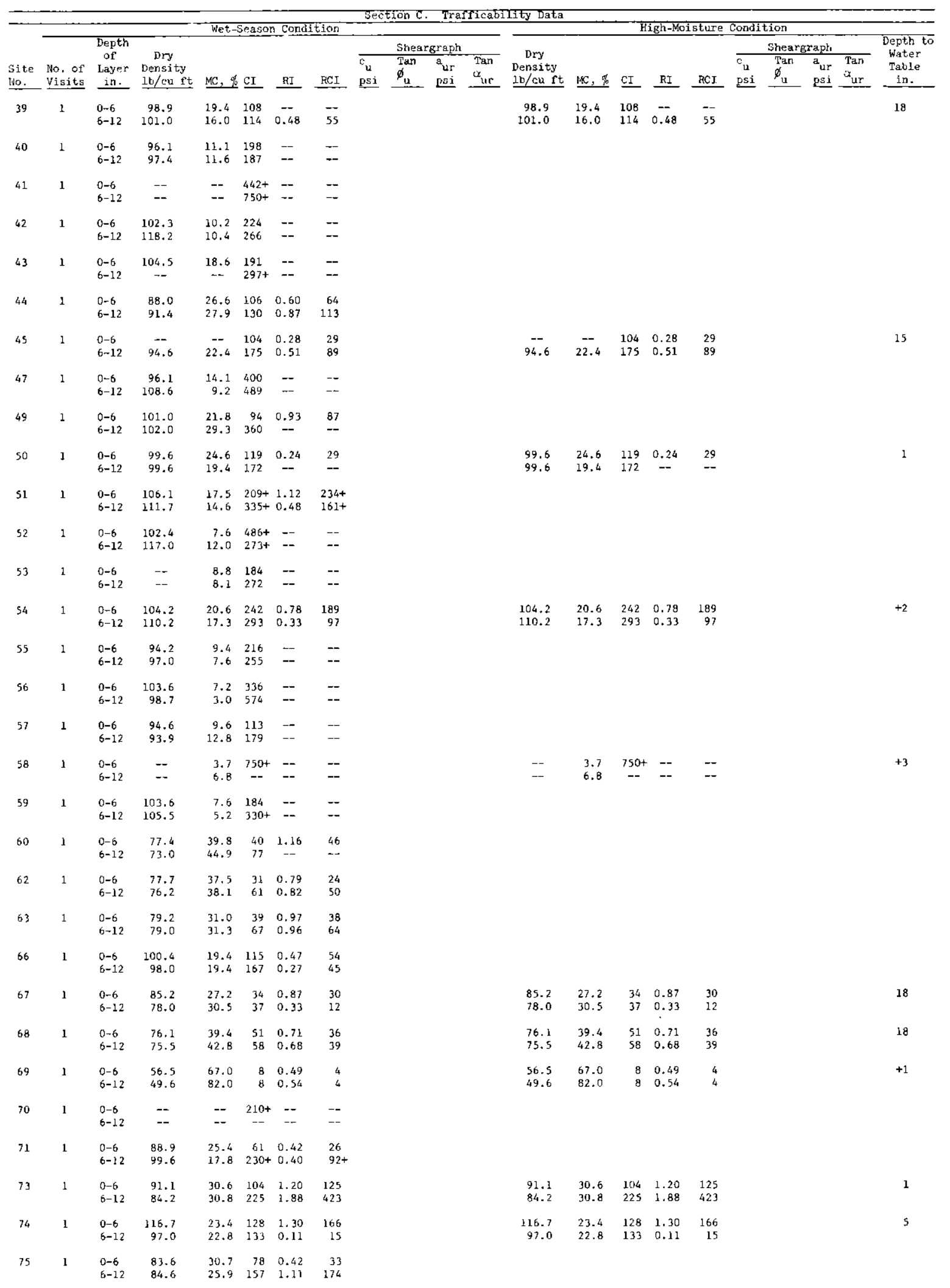




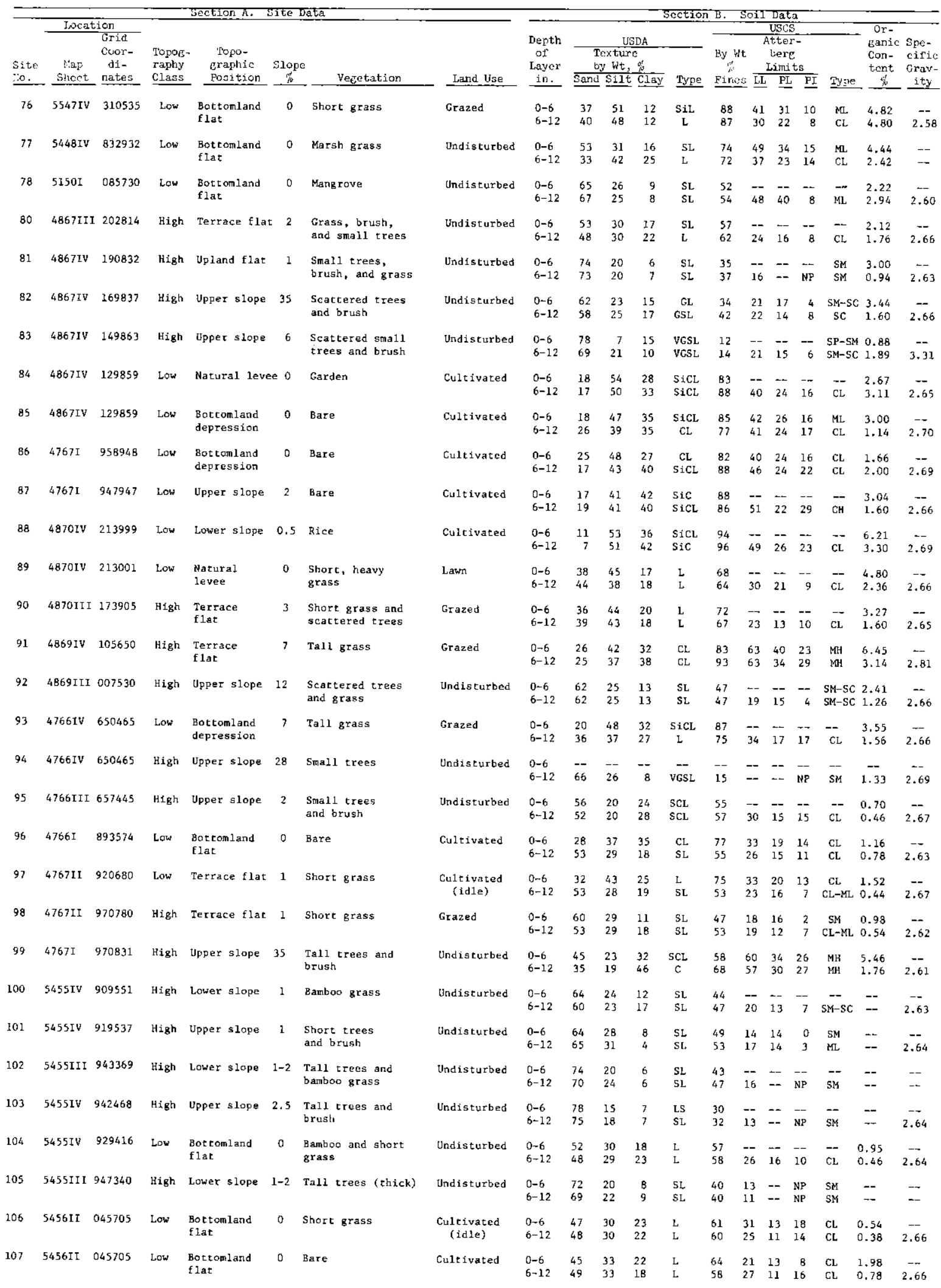




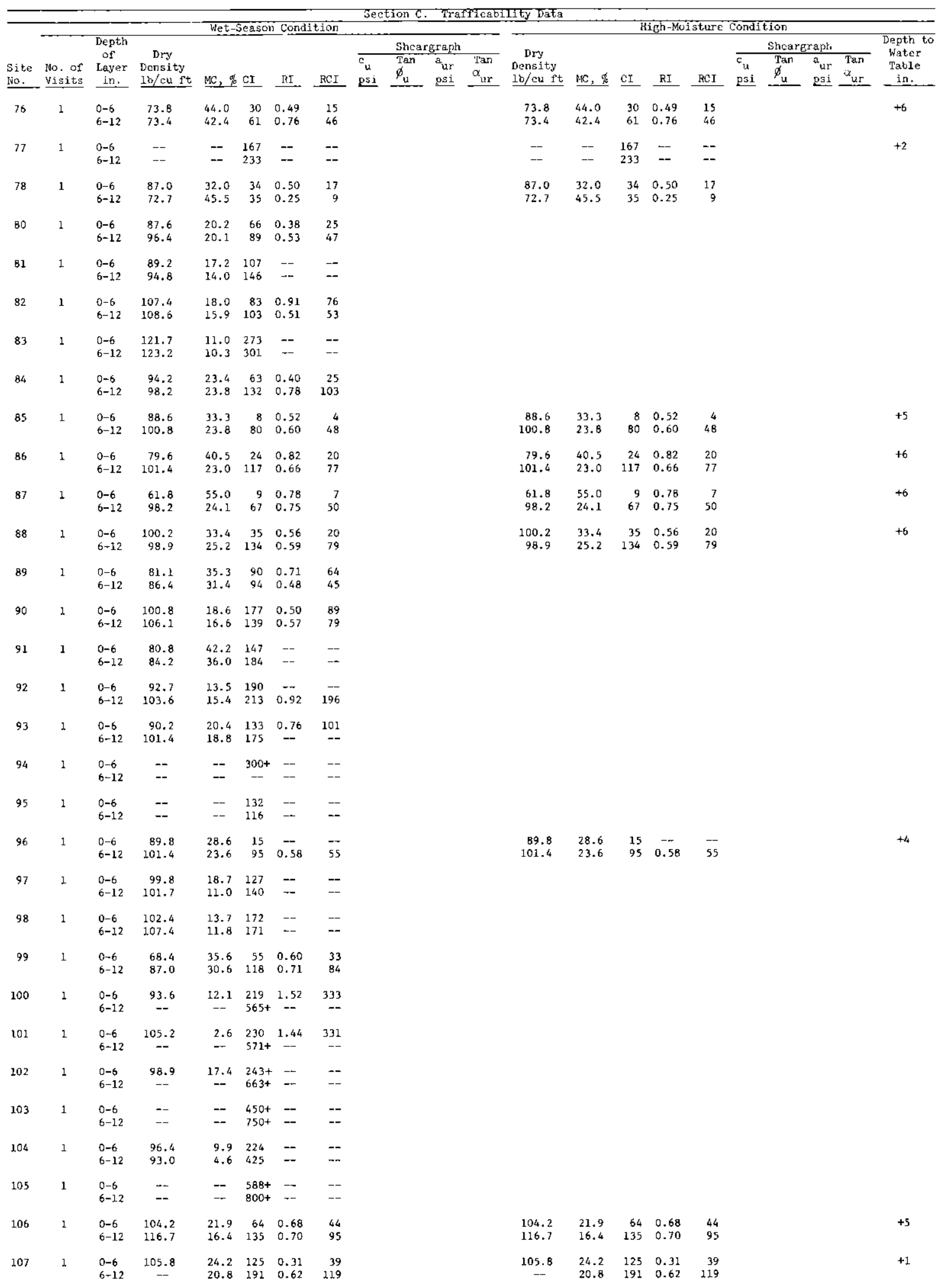


Table Al (Continued)

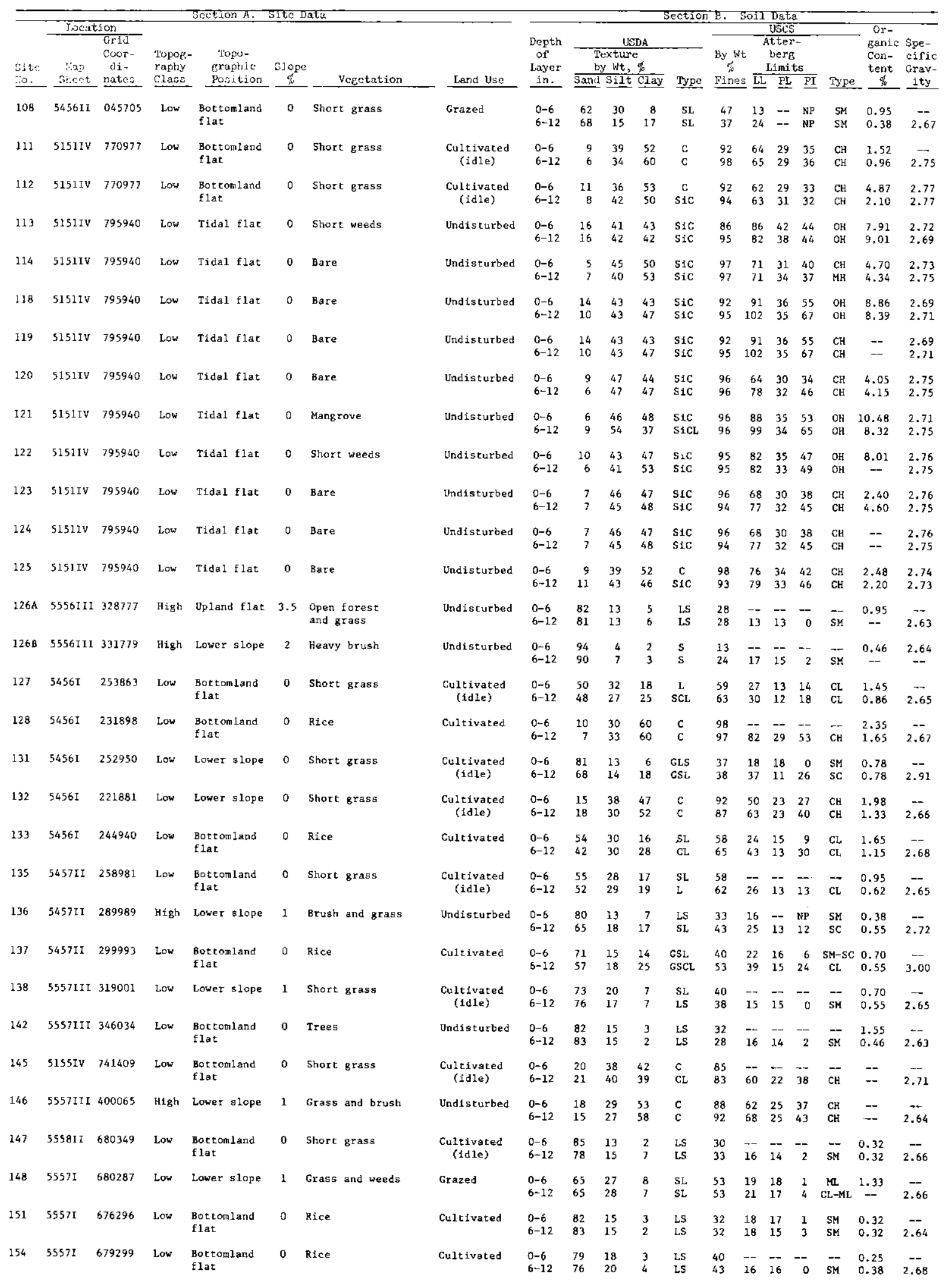




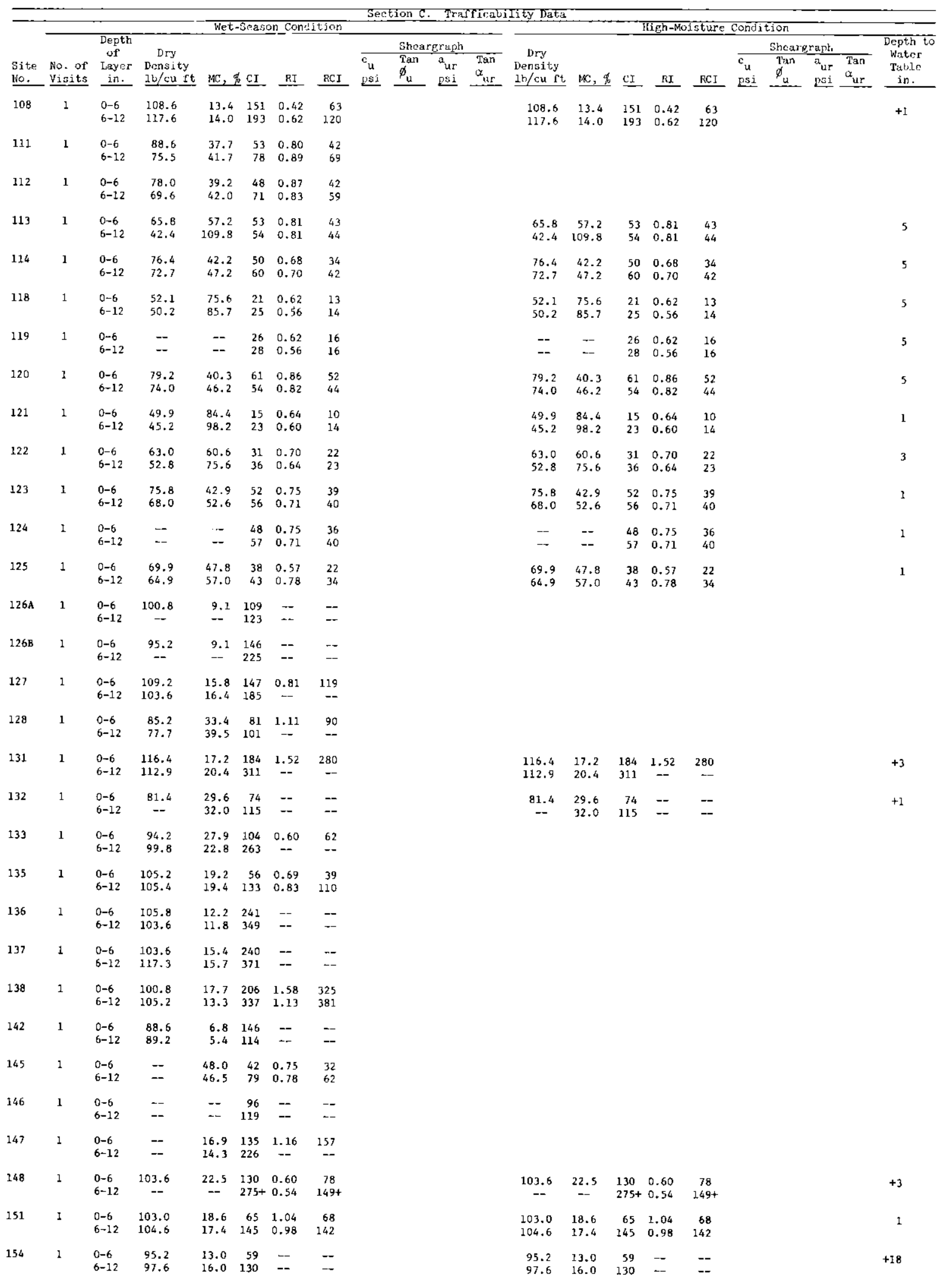




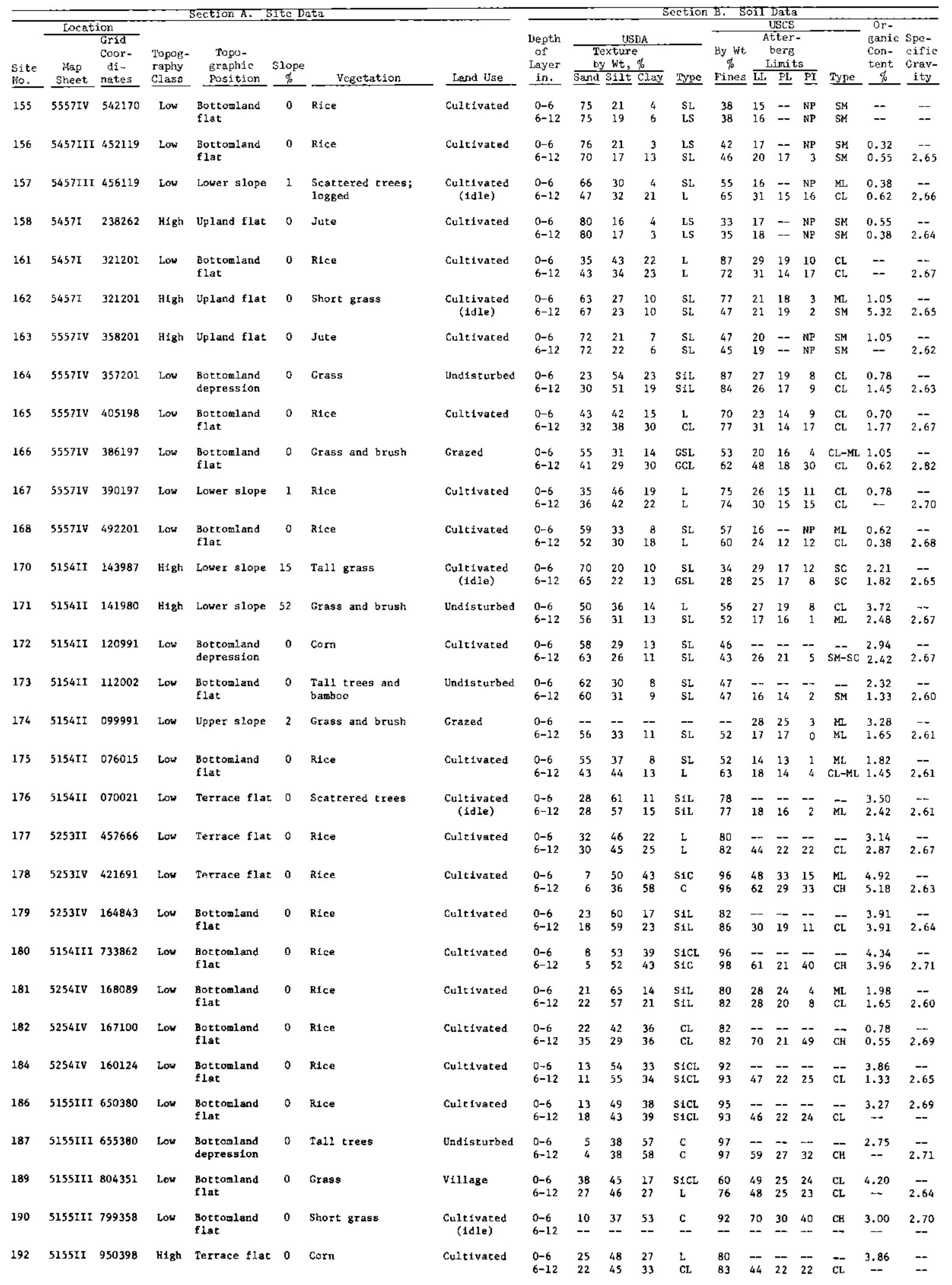




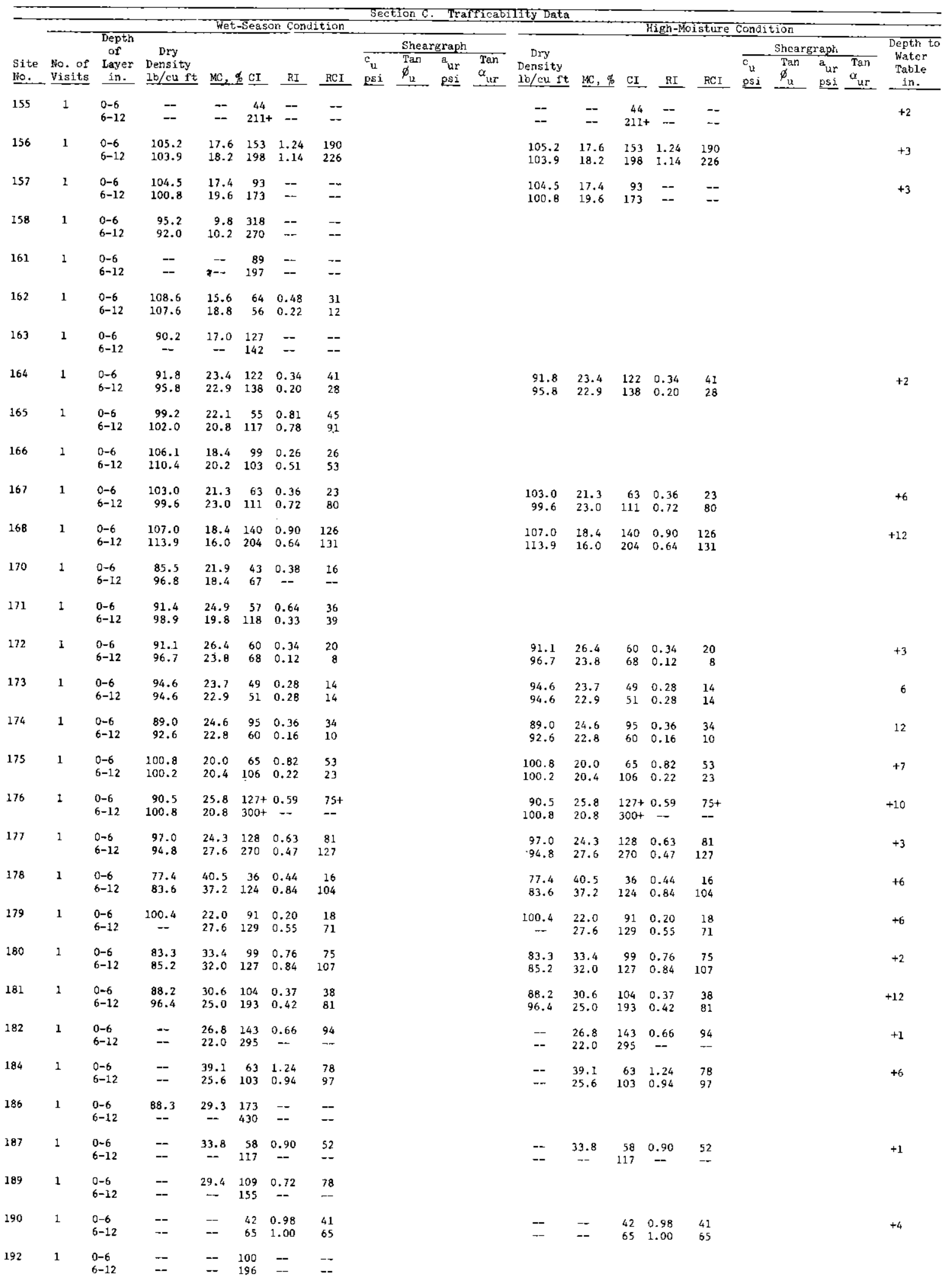


Table Al (Continued)

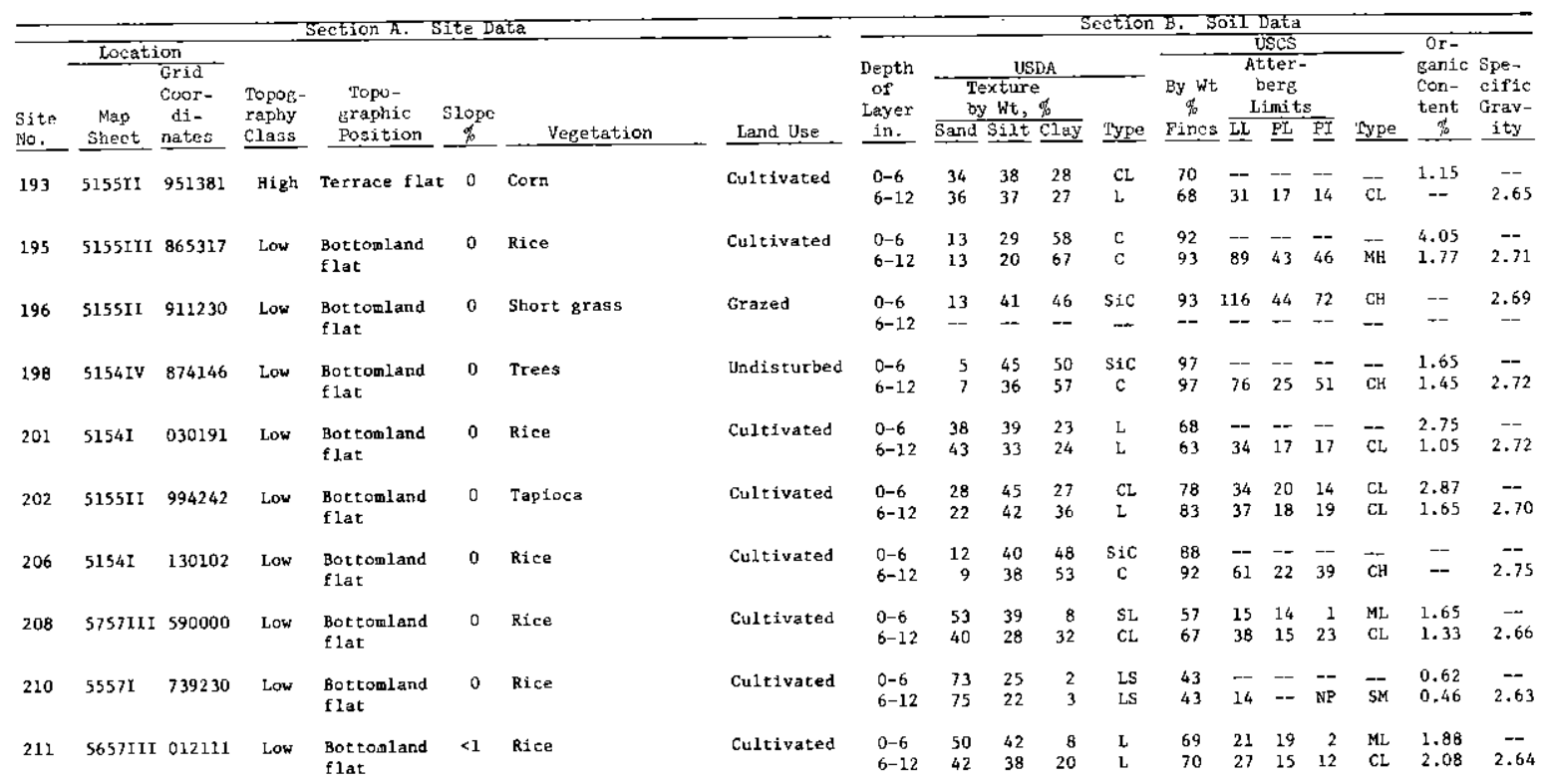




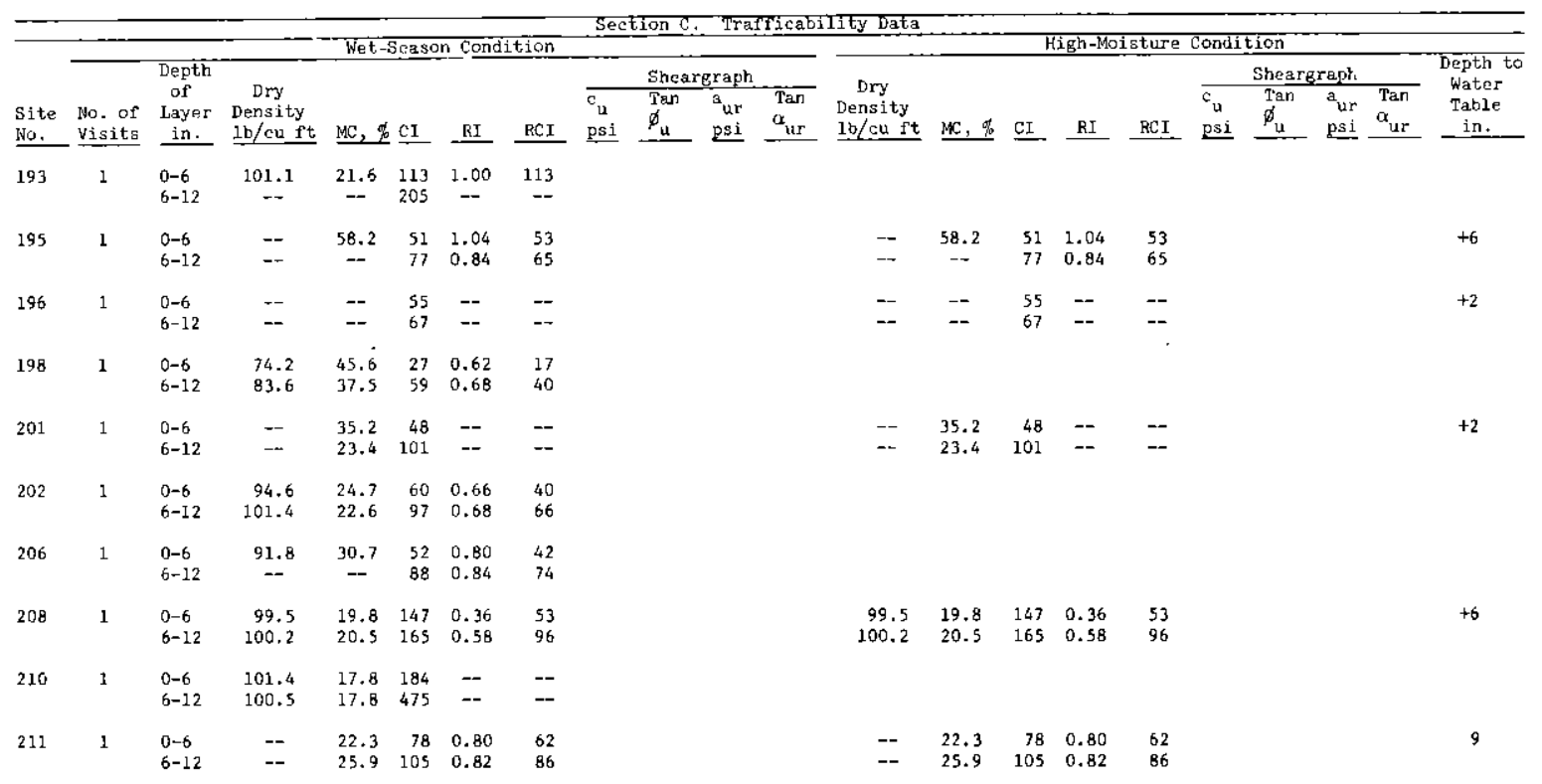


Table A2

Trafficability Classification Study

Sumary of Site, Soil, and Trafficobility Data

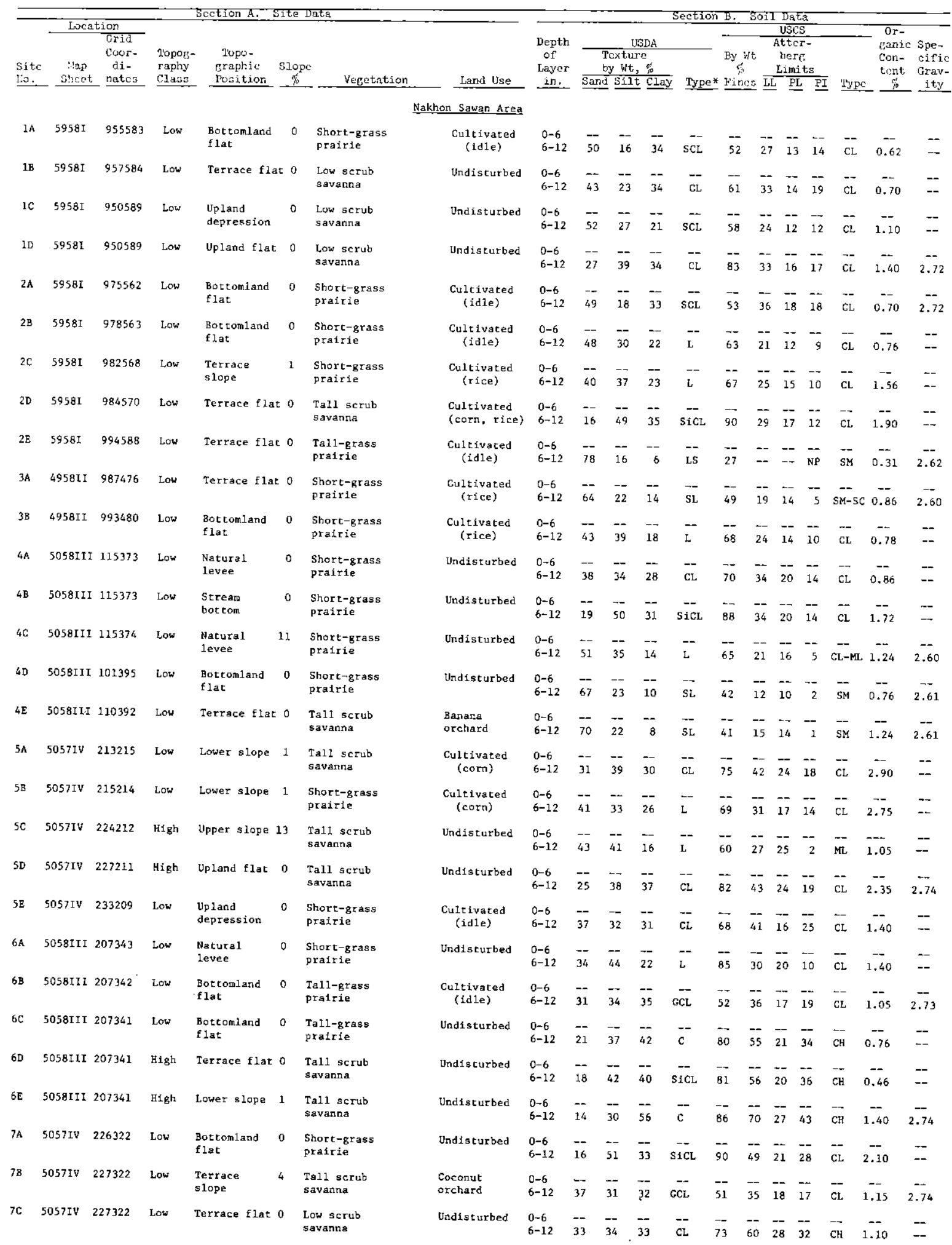

(Continued) 


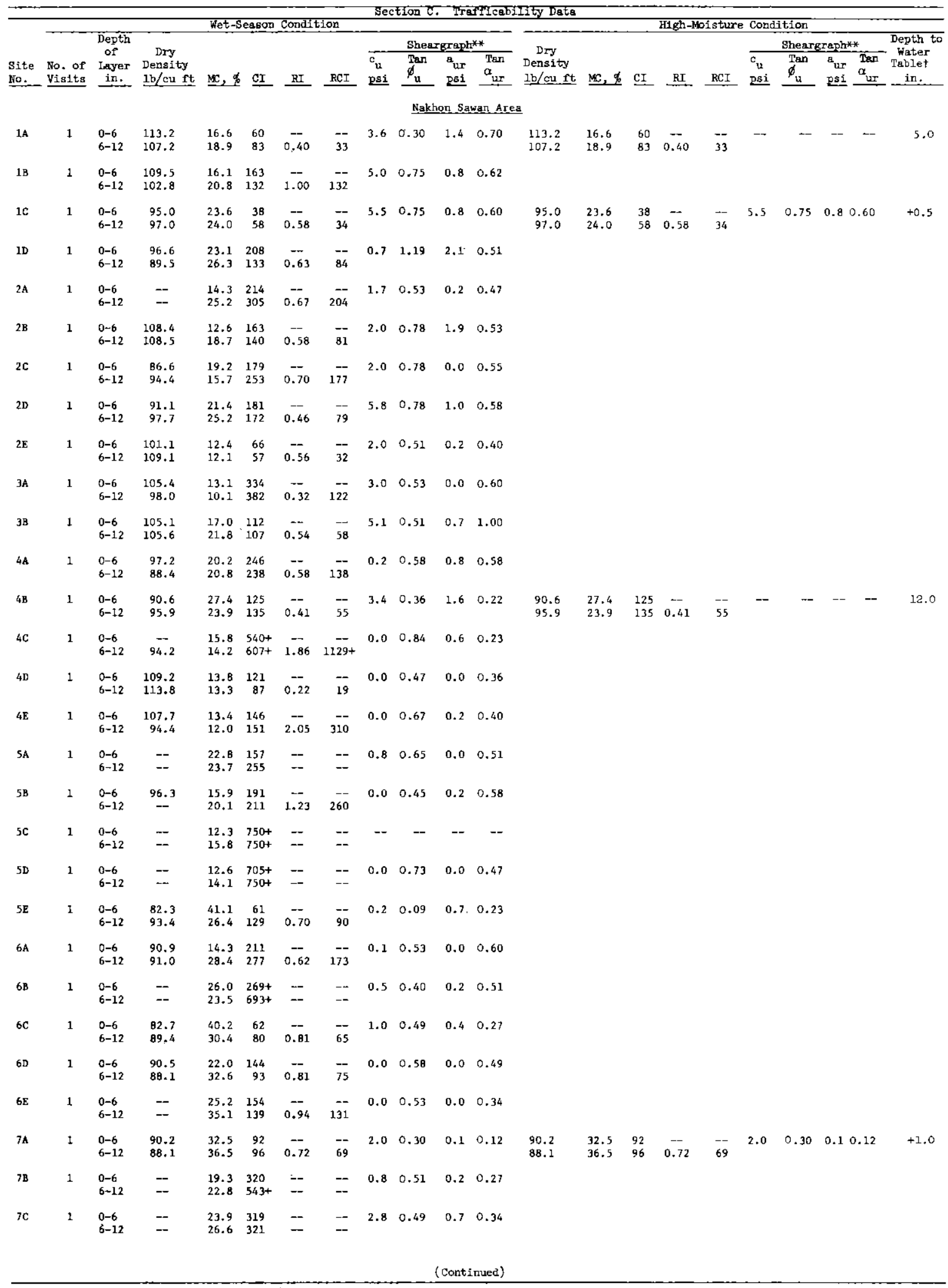

* $\varepsilon_{u}$, ultimate soil-to-soll cohesion; $\not \phi_{u}$, vltimate soil-to-soil angle of internal friction; a

$\alpha_{u r}$, ultimate soil-to-rubber angle of friction.

+ Plus $(+)$ denotes depth of water above surface. 


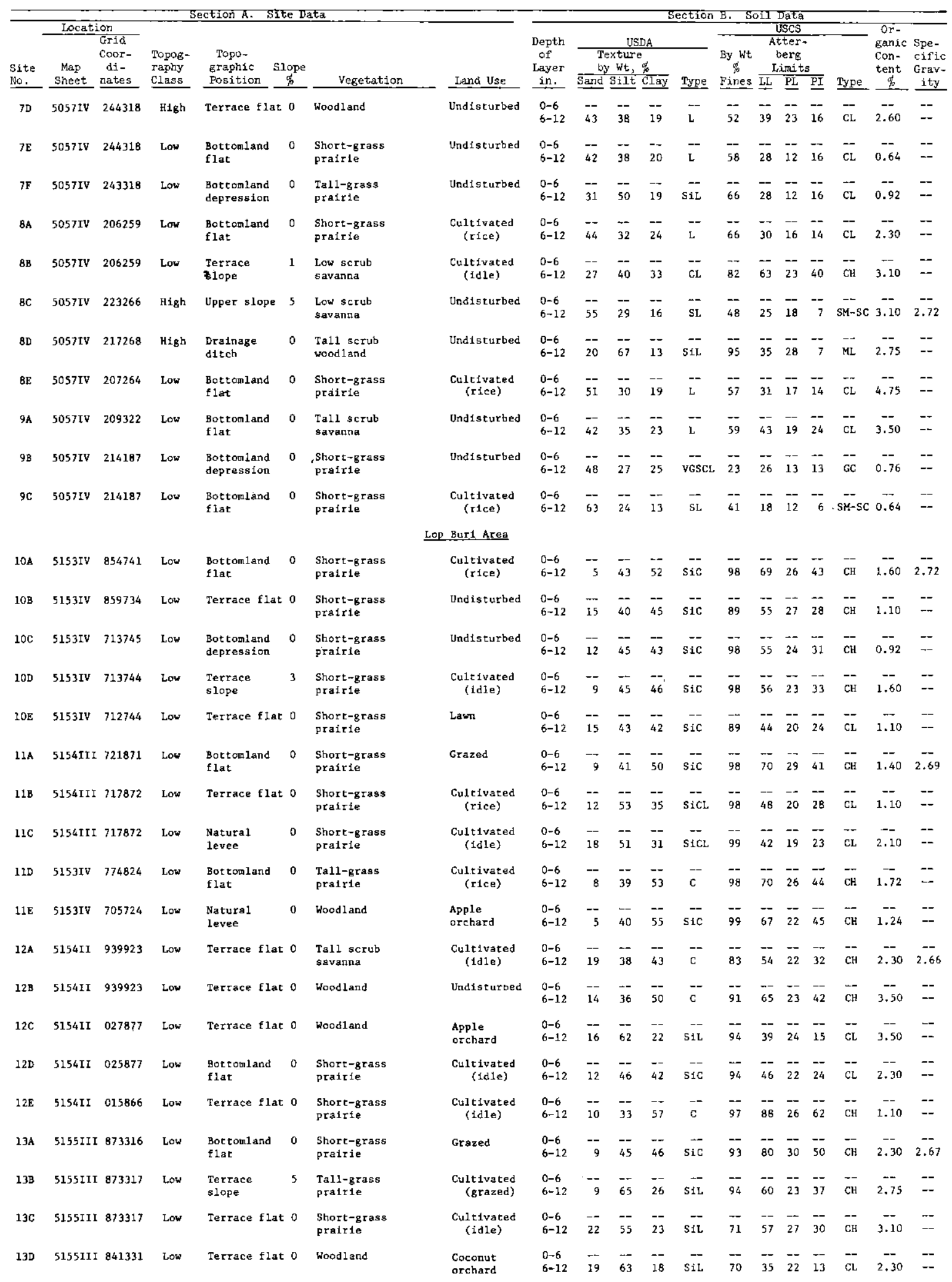




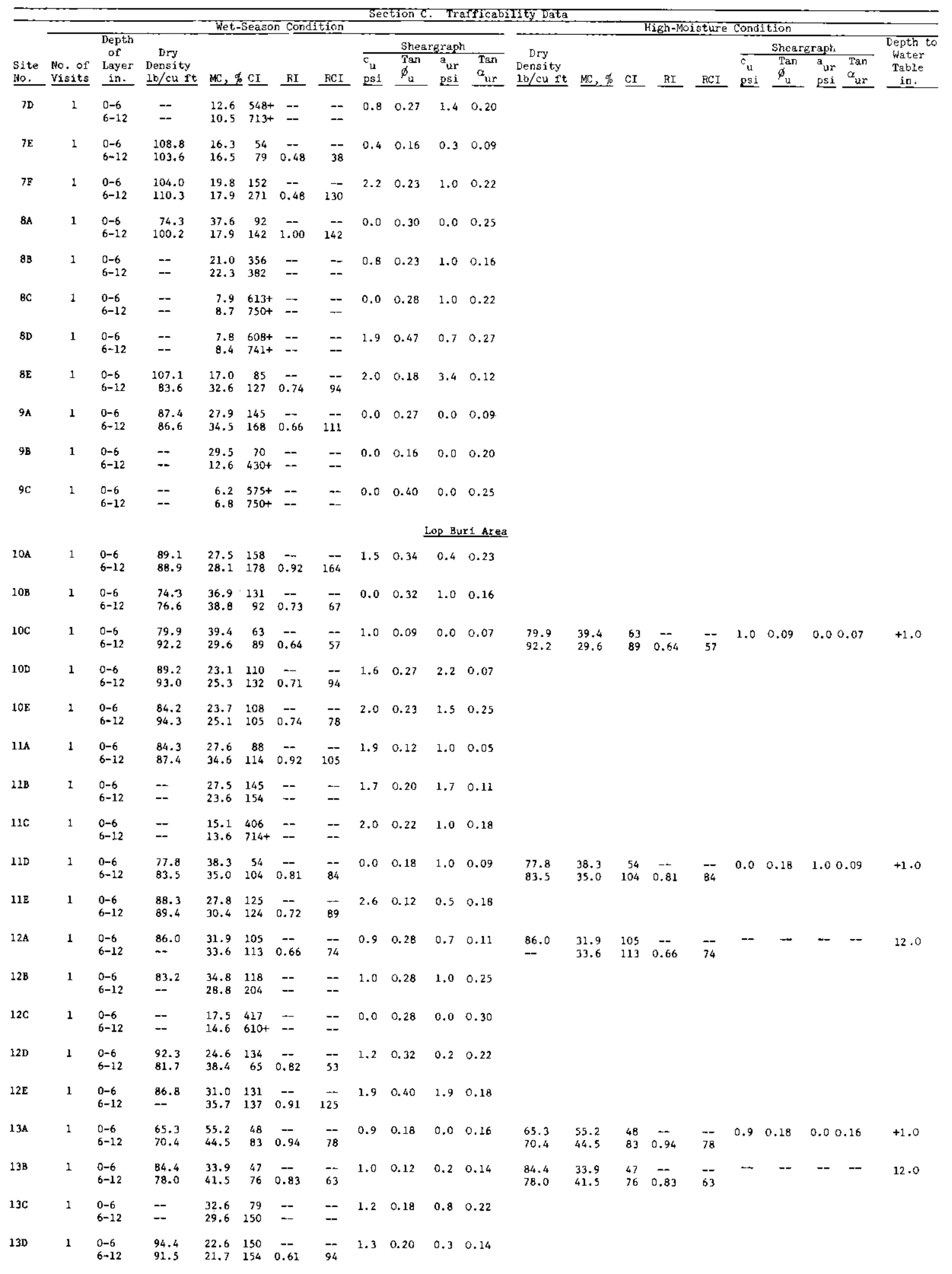




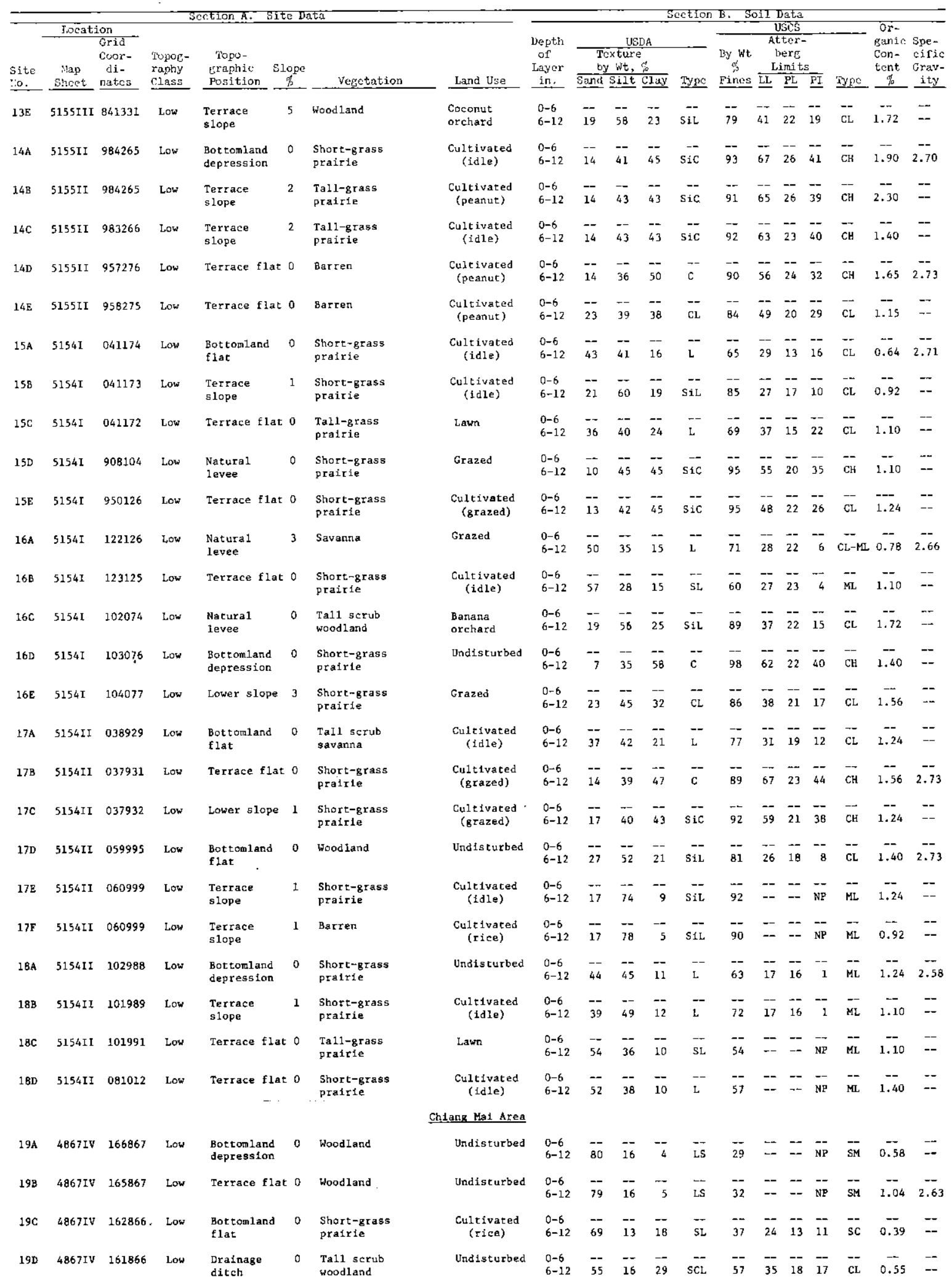




\begin{tabular}{|c|c|c|c|c|c|c|c|c|c|c|c|c|c|c|c|c|c|c|c|c|}
\hline \multirow[b]{3}{*}{$\begin{array}{l}\text { Site } \\
\text { ito. } \\
\end{array}$} & \multirow[b]{3}{*}{$\begin{array}{l}\text { No. of } \\
\text { Visits }\end{array}$} & \multirow{3}{*}{$\begin{array}{l}\text { Depth } \\
\text { of } \\
\text { Layer } \\
\text { in. } \\
\end{array}$} & \multirow{3}{*}{$\begin{array}{c}\text { Dry } \\
\text { Density } \\
\text { Lb/cu ft }\end{array}$} & \multicolumn{4}{|c|}{ Wet-Season_Condition } & \multirow{2}{*}{\multicolumn{4}{|c|}{ Shcargraph }} & \multirow{2}{*}{\multicolumn{8}{|c|}{$\frac{\text { High Moisture Condition }}{\text { Sheargrapk }}$}} & \multirow{3}{*}{$\begin{array}{l}\text { Depth to } \\
\text { Water } \\
\text { Table } \\
\text { in. } \\
\end{array}$} \\
\hline & & & & \multirow{2}{*}{\multicolumn{2}{|c|}{$\underline{M C}, \% \underline{C I}$}} & \multirow[b]{2}{*}{$\underline{R I}$} & \multirow[b]{2}{*}{$\mathrm{RCI}$} & & & & & & & & & & & & \\
\hline & & & & & & & & \multirow{2}{*}{$\begin{array}{l}\mathrm{c} \\
\mathrm{psi} \\
\mathrm{psi} \\
1.5\end{array}$} & \multirow{2}{*}{$\frac{\phi_{u}}{0.32}$} & \multirow{2}{*}{ 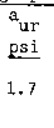 } & \multirow{2}{*}{$\begin{array}{c}\frac{\begin{array}{c}\text { Tan } \\
\alpha_{\text {ur }}\end{array}}{} \\
0.14\end{array}$} & $\begin{array}{l}\text { Dry } \\
\text { Density } \\
1 \mathrm{~b} / \mathrm{cu} \mathrm{ft}\end{array}$ & $\underline{M C,}$ & $\underline{\mathrm{CI}}$ & RI & $\mathrm{RCY}$ & \multirow[t]{2}{*}{$\begin{array}{l}c_{u} \\
\text { psi. }\end{array}$} & $\begin{array}{c}\operatorname{Tan}_{\mathrm{u}} \\
\mathrm{P}_{\mathrm{u}}\end{array}$ & $\begin{array}{ll}a_{u r} & \text { Tan } \\
\text { psi } & \alpha_{u r} \\
\end{array}$ & \\
\hline $13 \mathrm{E}$ & 1 & $\begin{array}{l}0-6 \\
6-12\end{array}$ & $\begin{array}{l}91.5 \\
93.8\end{array}$ & $\begin{array}{l}25.7 \\
22.9\end{array}$ & $\begin{array}{l}212 \\
112\end{array}$ & $--\overline{0}$ & $\overline{80}$ & & & & & & & & & & & & & \\
\hline $14 \mathrm{~A}$ & $x$ & $\begin{array}{l}0-6 \\
6-12\end{array}$ & $\begin{array}{l}81.4 \\
84.6\end{array}$ & $\begin{array}{l}36.3 \\
34.6\end{array}$ & $\begin{array}{r}30 \\
100\end{array}$ & $\overline{0.92}$ & $\overline{92}$ & 0.4 & 0.22 & 1.2 & 0.09 & $\begin{array}{l}81.4 \\
84.6\end{array}$ & $\begin{array}{l}36.3 \\
34.6\end{array}$ & $\begin{array}{r}30 \\
100\end{array}$ & 0.92 & $\overline{92}$ & 0.4 & 0.22 & 1.20 .09 & +3.0 \\
\hline $14 \mathrm{~B}$ & 1 & $\begin{array}{l}0-6 \\
6-12\end{array}$ & $\begin{array}{l}83.9 \\
85.6\end{array}$ & $\begin{array}{l}32.4 \\
30.4\end{array}$ & $\begin{array}{r}58 \\
133\end{array}$ & $\overline{1 .-16}$ & $\overline{154}$ & 1.2 & 0.22 & 1.6 & 0.14 & & & & & & & & & \\
\hline $14 \mathrm{C}$ & 1 & $\begin{array}{l}0-6 \\
6-12\end{array}$ & 86.8 & $\begin{array}{l}30.5 \\
29.4\end{array}$ & $\begin{array}{r}67 \\
151\end{array}$ & --- & $\overline{124}$ & 2.0 & 0.25 & 1.0 & 0.16 & & & & & & & & & \\
\hline 14D & 1 & $\begin{array}{l}0-6 \\
6-12\end{array}$ & $\begin{array}{l}79.1 \\
81.0\end{array}$ & $\begin{array}{l}29.0 \\
30.5\end{array}$ & $\begin{array}{l}126 \\
195\end{array}$ & 0.82 & -- & 0.6 & 0.32 & 1.7 & 0.30 & & & & & & & & & \\
\hline 14E & 1 & $\begin{array}{l}0-6 \\
6-12\end{array}$ & $\begin{array}{l}88.6 \\
91.0\end{array}$ & $\begin{array}{l}25.9 \\
23.9\end{array}$ & $\begin{array}{r}97 \\
190\end{array}$ & 0.76 & $\overline{144}$ & 0.4 & 0.45 & 0.0 & 0.28 & & & & & & & & & \\
\hline $15 \mathrm{~A}$ & 1 & $\begin{array}{l}0-6 \\
6-12\end{array}$ & $\begin{array}{l}106.8 \\
103.2\end{array}$ & $\begin{array}{l}19.0 \\
20.4\end{array}$ & $\begin{array}{r}76 \\
115\end{array}$ & $\overline{0.51}$ & $\overline{59}$ & 0.5 & 0.23 & 0.8 & 0.09 & $\begin{array}{l}106.8 \\
103.2\end{array}$ & $\begin{array}{l}19.0 \\
20.4\end{array}$ & $\begin{array}{r}76 \\
115\end{array}$ & 0.51 & $\overline{59}$ & 0.5 & 0.23 & 0.80 .09 & +1.0 \\
\hline 15B & 1 & $\begin{array}{l}0-6 \\
6-12\end{array}$ & $\begin{array}{l}99.9 \\
97.0\end{array}$ & $\begin{array}{l}19.5 \\
23.1\end{array}$ & $\begin{array}{r}97 \\
147\end{array}$ & 0.55 & $\overline{81}$ & 0.7 & 0.27 & 0.1 & 0.27 & & & & & & & & & \\
\hline $15 \mathrm{C}$ & 1 & $\begin{array}{l}0-6 \\
6-12\end{array}$ & $\begin{array}{r}93.9 \\
106.3\end{array}$ & $\begin{array}{l}31.8 \\
19.5\end{array}$ & $\begin{array}{l}125 \\
143\end{array}$ & $\overline{0.88}$ & $\overline{126}$ & 1.6 & 0.28 & 0.4 & 0.30 & & & & & & & & & \\
\hline 15D & 1 & $\begin{array}{l}0-6 \\
6-12\end{array}$ & $\begin{array}{l}92.7 \\
90.6\end{array}$ & $\begin{array}{l}26.6 \\
25.2\end{array}$ & $\begin{array}{l}100 \\
149\end{array}$ & 0.95 & $\overline{142}$ & 1.0 & 0.23 & 0.4 & 0.20 & & & & & & & & & \\
\hline $15 E$ & 1 & $\begin{array}{l}0-6 \\
6-12\end{array}$ & $\begin{array}{l}89.7 \\
92.5\end{array}$ & $\begin{array}{l}29.4 \\
26.5\end{array}$ & $\begin{array}{r}90 \\
147\end{array}$ & $\overline{--}$ & $\overline{137}$ & 1.8 & 0.23 & 0.0 & 0.32 & & & & & & & & & \\
\hline 16A & 1 & $\begin{array}{l}0-6 \\
6-12\end{array}$ & $\begin{array}{l}88.7 \\
89.4\end{array}$ & $\begin{array}{l}21.5 \\
14.0\end{array}$ & $\begin{array}{l}240 \\
390\end{array}$ & 0.95 & $\overline{370}$ & 1.0 & 0.40 & 0.0 & 0.32 & & & & & & & & & \\
\hline 16B & 1 & $\begin{array}{l}0-6 \\
6-12\end{array}$ & $\begin{array}{l}93.5 \\
92.3\end{array}$ & $\begin{array}{l}19.9 \\
15.9\end{array}$ & $\begin{array}{l}237 \\
356\end{array}$ & $\overline{1.07}$ & $\overrightarrow{381}$ & 0.0 & 0.49 & 0.0 & 0.30 & & & & & & & & & \\
\hline $16 \mathrm{C}$ & 1 & $\begin{array}{l}0-6 \\
6-12\end{array}$ & $\begin{array}{l}82.8 \\
90.5\end{array}$ & $\begin{array}{l}30.3 \\
27.0\end{array}$ & $\begin{array}{l}65 \\
70\end{array}$ & $-\overline{0.61}$ & -- & 1.3 & 0.32 & 0.0 & 0.36 & & & & & & & & & \\
\hline 16D & 1 & $\begin{array}{l}0-6 \\
6-12\end{array}$ & $\begin{array}{l}88.0 \\
87.6\end{array}$ & $\begin{array}{l}28.8 \\
31.5\end{array}$ & $\begin{array}{l}55 \\
95\end{array}$ & $\overline{0.49}$ & -- & 0.0 & 0.18 & 0.0 & 0.12 & $\begin{array}{l}88.0 \\
87.6\end{array}$ & $\begin{array}{l}28.8 \\
31.5\end{array}$ & $\begin{array}{l}55 \\
95\end{array}$ & $0 .-49$ & -- & 0.0 & 0.18 & 0.00 .12 & +3.0 \\
\hline $16 \mathrm{E}$ & 1 & $\begin{array}{l}0-6 \\
6-12\end{array}$ & $\begin{array}{l}94.5 \\
91.6\end{array}$ & $\begin{array}{l}21.9 \\
25.2\end{array}$ & $\begin{array}{l}244 \\
201\end{array}$ & $\overline{0.68}$ & $\begin{array}{r}-- \\
137\end{array}$ & 0.1 & 0.51 & 0.0 & 0.22 & & & & & & & & & \\
\hline $17 \mathrm{~A}$ & 1 & $\begin{array}{l}0-6 \\
6-12\end{array}$ & $\begin{array}{l}99.4 \\
94.4\end{array}$ & $\begin{array}{l}21.3 \\
25.5\end{array}$ & $\begin{array}{l}105 \\
116\end{array}$ & $\overline{0.56}$ & -- & 0.7 & 0.30 & 0.0 & 0.11 & $\begin{array}{l}99.4 \\
94.4\end{array}$ & $\begin{array}{l}21.3 \\
25.5\end{array}$ & $\begin{array}{l}105 \\
116\end{array}$ & -- & -- & -- & -- & $--\quad--$ & 7.0 \\
\hline $17 \mathrm{~B}$ & 1 & $\begin{array}{l}0-6 \\
6-12\end{array}$ & $\begin{array}{l}94.8 \\
90.0\end{array}$ & $\begin{array}{l}22.9 \\
28.0\end{array}$ & $\begin{array}{l}192 \\
198\end{array}$ & 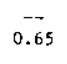 & $\overline{129}$ & 1.9 & 0.36 & 2.4 & 0.09 & & & & & & & & & \\
\hline $17 \mathrm{c}$ & 1 & $\begin{array}{l}0-6 \\
6-12\end{array}$ & $\begin{array}{l}97.3 \\
94.3\end{array}$ & $\begin{array}{l}23.6 \\
28.5\end{array}$ & $\begin{array}{r}83 \\
106\end{array}$ & $\overline{0.39}$ & -- & 0.5 & 0.18 & 1.1 & 0.18 & $\begin{array}{l}97.3 \\
94.3\end{array}$ & $\begin{array}{l}23.6 \\
28.5\end{array}$ & $\begin{array}{r}83 \\
106\end{array}$ & 0.39 & 41 & 0.5 & 0.18 & 1.10 .18 & +1.0 \\
\hline $17 \mathrm{D}$ & 1 & $\begin{array}{l}0-6 \\
6-12\end{array}$ & $\begin{array}{l}79.9 \\
96.0\end{array}$ & $\begin{array}{l}30.7 \\
18.6\end{array}$ & $\begin{array}{l}178 \\
165\end{array}$ & $\overline{0 .}$ & -- & 0.7 & 0.45 & 0.0 & 0.27 & & & & & & & & & \\
\hline $17 E$ & 1 & $\begin{array}{l}0-6 \\
6-12\end{array}$ & -- & $\begin{array}{l}14.8 \\
13.3\end{array}$ & $\begin{array}{l}647 \\
750+\end{array}$ & -- & $\begin{array}{l}-- \\
--\end{array}$ & 0.0 & 0.36 & 0.0 & 0.28 & & & & & & & & & \\
\hline $17 \mathrm{~F}$ & 1 & $\begin{array}{l}0-6 \\
6-12\end{array}$ & -- & $\begin{array}{l}43.3 \\
21.0\end{array}$ & $\begin{array}{l}437+ \\
750+\end{array}$ & -- & $=$ & 0.0 & 0.47 & 1.8 & 0.32 & $=-$ & $\begin{array}{l}43.3 \\
21.0\end{array}$ & $\begin{array}{l}437+ \\
750+\end{array}$ & -- & -- & 0.0 & 0.47 & 1.80 .32 & +3.0 \\
\hline $18 \mathrm{~A}$ & 1 & $\begin{array}{l}0-6 \\
6-12\end{array}$ & $\begin{array}{l}97.5 \\
92.8\end{array}$ & $\begin{array}{l}19.6 \\
25.5\end{array}$ & $\begin{array}{l}221 \\
205\end{array}$ & $\overline{0.15}$ & $\overline{31}$ & 2.0 & 0.22 & 0.0 & 0.25 & $\begin{array}{l}97.5 \\
92.8\end{array}$ & $\begin{array}{l}19.6 \\
25.5\end{array}$ & $\begin{array}{l}221 \\
205\end{array}$ & $\begin{array}{c}-- \\
0.15\end{array}$ & 31 & -- & -- & $--\quad--$ & 9.0 \\
\hline $18 \mathrm{~B}$ & 1 & $\begin{array}{l}0-6 \\
6-12\end{array}$ & $\begin{array}{l}98.6 \\
97.9\end{array}$ & $\begin{array}{l}19.3 \\
16.3\end{array}$ & $\begin{array}{l}221 \\
379\end{array}$ & $--\overline{0.48}$ & $-\overline{182}$ & 0.0 & 0.32 & 0.0 & 0.32 & & & & & & & & & \\
\hline $18 \mathrm{C}$ & 1 & $\begin{array}{l}0-6 \\
6-12\end{array}$ & $\begin{array}{l}93.7 \\
92.8\end{array}$ & $\begin{array}{l}15.9 \\
15.9\end{array}$ & $\begin{array}{l}423 \\
433\end{array}$ & $\overline{1.66}$ & $\overline{719}$ & 0.0 & 0.42 & 0.8 & 0.25 & & & & & & & & & \\
\hline $18 \mathrm{D}$ & 1 & $\begin{array}{l}0-6 \\
6-12\end{array}$ & $\begin{array}{r}97.6 \\
102.6\end{array}$ & $\begin{array}{l}20.0 \\
18.9\end{array}$ & $\begin{array}{l}261 \\
687+\end{array}$ & 0.46 & $\overline{316+}$ & 0.0 & 0.27 & 0.0 & 0.20 & $\begin{array}{r}97.6 \\
102.6\end{array}$ & $\begin{array}{l}20.0 \\
18.9\end{array}$ & $\begin{array}{l}261 \\
687+\end{array}$ & $\overline{0 .-}$ & -- & 0.0 & 0.27 & 0.00 .20 & +0.5 \\
\hline & & & & & & & & & & ians - & Mai Aree & & & & & & & & & \\
\hline $19 \mathrm{~A}$ & 1 & $\begin{array}{l}0-6 \\
6-12\end{array}$ & $\begin{array}{r}99.4 \\
102.1\end{array}$ & $\begin{array}{l}16.5 \\
16.1\end{array}$ & $\begin{array}{r}96 \\
175\end{array}$ & -- & $-\overline{9}$ & 0.0 & 0.27 & 0.7 & 0.14 & $\begin{array}{r}99.4 \\
102.1\end{array}$ & $\begin{array}{l}16.5 \\
16.1\end{array}$ & $\begin{array}{r}96 \\
175\end{array}$ & $0 .-53$ & 93 & 0.0 & 0.27 & 0.70 .14 & 0.0 \\
\hline $19 \mathrm{~B}$ & 1 & $\begin{array}{l}0-6 \\
6-12\end{array}$ & $\begin{array}{l}92.5 \\
94.8\end{array}$ & $\begin{array}{l}18.7 \\
19.5\end{array}$ & $\begin{array}{l}155 \\
174\end{array}$ & -- & -- & 0.5 & 0.30 & 0.9 & 0.34 & & & & & & & & & \\
\hline $19 \mathrm{C}$ & 1 & $\begin{array}{l}0-6 \\
6-1.2\end{array}$ & $\begin{array}{r}86.2 \\
100.0\end{array}$ & $\begin{array}{l}46.0 \\
22.3\end{array}$ & $\begin{array}{l}128 \\
112\end{array}$ & $\overline{0.2 B}$ & $\overline{31}$ & -- & -- & -- & -- & $\begin{array}{r}86.2 \\
100.0\end{array}$ & $\begin{array}{l}46.0 \\
22.3\end{array}$ & $\begin{array}{l}128 \\
112\end{array}$ & $\overline{0.28}$ & -- & -- & - & -- $\quad--$ & +12.0 \\
\hline 190 & 1 & $\begin{array}{l}0-6 \\
6-12\end{array}$ & $\begin{array}{l}104.9 \\
103.1\end{array}$ & $\begin{array}{l}21.4 \\
22.9\end{array}$ & $\begin{array}{r}76 \\
156\end{array}$ & $\overline{0.59}$ & -- & 1.6 & 0.42 & 0.0 & 0.23 & $\begin{array}{l}104.9 \\
103.1\end{array}$ & $\begin{array}{l}21.4 \\
22.9\end{array}$ & $\begin{array}{r}76 \\
156\end{array}$ &.-- & $\overline{92}$ & 1.6 & 0.42 & 0.00 .23 & +6.0 \\
\hline
\end{tabular}




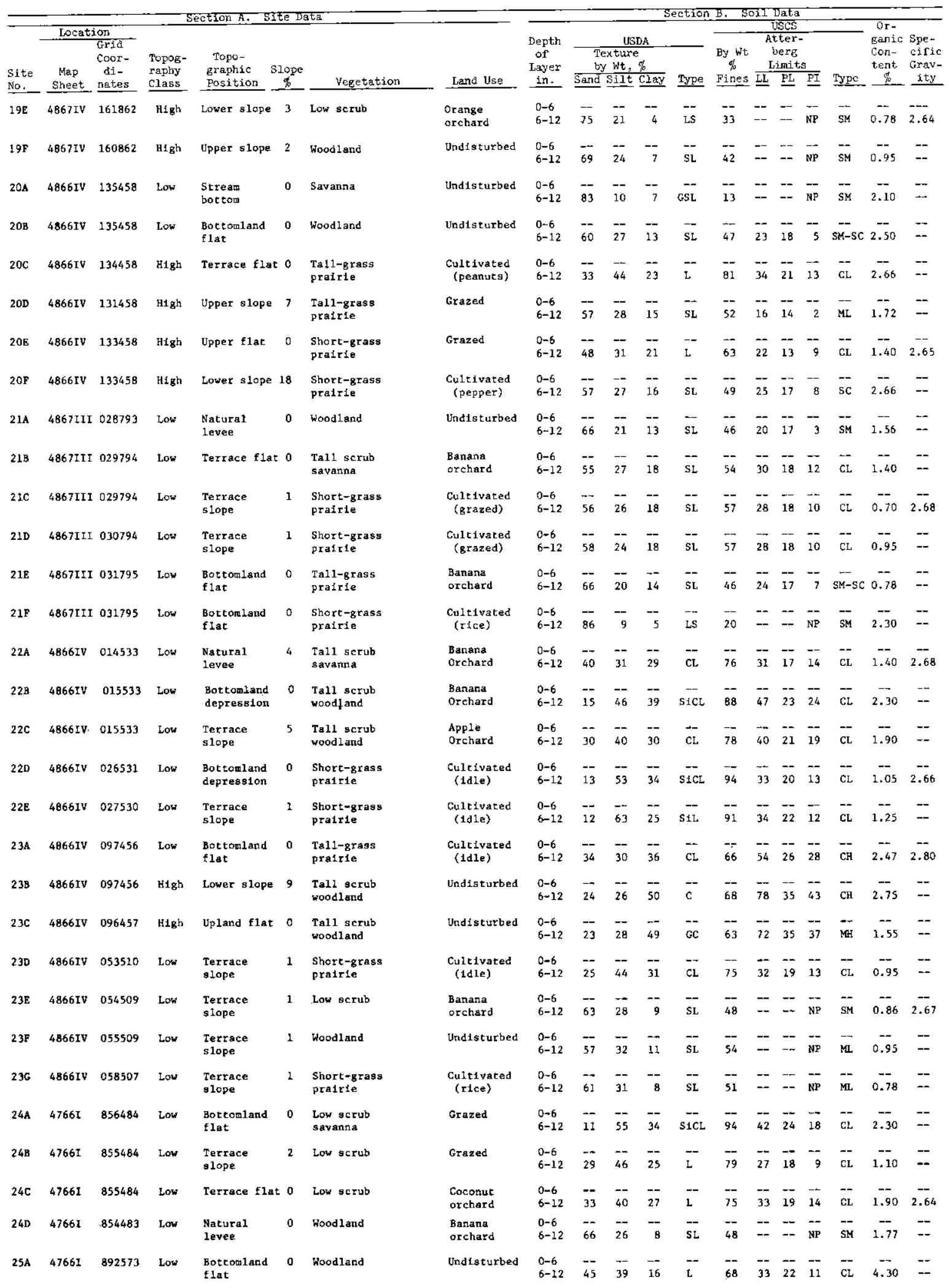




\begin{tabular}{|c|c|c|c|c|c|c|c|c|c|c|c|c|c|c|c|c|c|c|c|c|}
\hline \multirow[b]{3}{*}{$\begin{array}{l}\text { Site } \\
\text { No. }\end{array}$} & \multirow{3}{*}{$\begin{array}{l}\text { No. of } \\
\text { Visits }\end{array}$} & \multirow{3}{*}{$\begin{array}{l}\text { Depth } \\
\text { of } \\
\text { Layer } \\
\text { in. } \\
\end{array}$} & \multicolumn{9}{|c|}{ Wet-Season Condition } & \multirow{2}{*}{\multicolumn{8}{|c|}{$\begin{array}{l}\text { High-Moistiure condition } \\
\text { Sheargraph. }\end{array}$}} & \multirow{3}{*}{$\begin{array}{l}\text { Depth to } \\
\text { Water } \\
\text { Table } \\
\text { in. } \\
\end{array}$} \\
\hline & & & \multirow[b]{2}{*}{$\begin{array}{l}\text { Dry } \\
\text { Density } \\
\text { lb/cu ft }\end{array}$} & \multirow[b]{2}{*}{$M C, D$} & \multirow[b]{2}{*}{ CI } & \multirow[b]{2}{*}{ RI } & \multirow[b]{2}{*}{$\mathrm{RCI}$} & \multicolumn{4}{|c|}{ Sheargraph } & & & & & & & & & \\
\hline & & & & & & & & $\begin{array}{l}c_{u} \\
\text { ssi }\end{array}$ & $\begin{array}{l}T_{u} \\
\phi_{u} \\
\end{array}$ & $\begin{array}{l}\mathrm{aur} \\
\underline{\mathrm{psi}}\end{array}$ & $\begin{array}{l}\text { Tann } \\
\alpha_{u r} \\
\end{array}$ & $\begin{array}{l}\text { Dry } \\
\text { Density } \\
\text { 1b/cu ft } \\
\end{array}$ & $\underline{\mathrm{MC}, \boldsymbol{\phi}}$ & $\underline{C I}$ & $\mathrm{RI}$ & $\mathrm{RCI}$ & $\begin{array}{l}c_{u} \\
\text { psi }\end{array}$ & $\begin{array}{l}\operatorname{Tan} \\
\not \\
\end{array}$ & 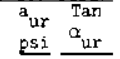 & \\
\hline $19 \mathrm{E}$ & 1 & $\begin{array}{l}0-6 \\
6-12\end{array}$ & $\begin{array}{r}94.9 \\
101.3\end{array}$ & $\begin{array}{l}11.7 \\
15.1\end{array}$ & $\begin{array}{l}117 \\
197\end{array}$ & $\overline{0.86}$ & $\overline{169}$ & 0.0 & 0.32 & 0.3 & 0.27 & & & & & & & & & \\
\hline $19 F$ & 1 & $\begin{array}{l}0-6 \\
6-12\end{array}$ & $\begin{array}{l}88.2 \\
97.6\end{array}$ & $\begin{array}{l}15.2 \\
13.3\end{array}$ & $\begin{array}{l}110 \\
116\end{array}$ & $\overline{0.98}$ & $\overline{114}$ & 1.5 & 0.34 & 1.4 & 0.32 & & & & & & & & & \\
\hline $20 \mathrm{~A}$ & 1 & $\begin{array}{l}0-6 \\
6-12\end{array}$ & $\begin{array}{l}89.0 \\
--\end{array}$ & $\begin{array}{l}23.3 \\
19.9\end{array}$ & $\begin{array}{r}52 \\
118\end{array}$ & -- & $\overline{--}$ & 1.0 & 0.14 & 0.8 & 0.20 & $\begin{array}{l}89.0 \\
\because-\end{array}$ & $\begin{array}{l}23.3 \\
19.9\end{array}$ & $\begin{array}{r}52 \\
118\end{array}$ & $\ddot{z}$ & -- & - & -- & $--\quad--$ & 2.0 \\
\hline $20 B$ & 1 & $\begin{array}{l}0-6 \\
6-12\end{array}$ & $\begin{array}{l}85.7 \\
94.4\end{array}$ & $\begin{array}{l}22.8 \\
14.6\end{array}$ & $\begin{array}{l}118 \\
199\end{array}$ & $\overline{1.01}$ & $\overline{201}$ & 0.9 & 0.38 & 0.1 & 0.51 & & & & & & & & & \\
\hline $20 \mathrm{C}$ & 1 & $\begin{array}{l}0-6 \\
6-12\end{array}$ & $\begin{array}{l}90.0 \\
93.8\end{array}$ & $\begin{array}{l}24.4 \\
23.9\end{array}$ & $\begin{array}{l}100 \\
115\end{array}$ & 0.56 & $\overline{64}$ & 1.6 & 0.36 & 0.4 & 0.30 & & & & & & & & & \\
\hline 20D & 1 & $\begin{array}{l}0-6 \\
6-12\end{array}$ & $\begin{array}{r}92.4 \\
104.0\end{array}$ & $\begin{array}{l}26.8 \\
17.6\end{array}$ & $\begin{array}{l}116 \\
156\end{array}$ & $\overline{0.32}$ & $\overline{50}$ & 0.4 & 0.30 & 0.7 & 0.23 & & & & & & & & & \\
\hline 208 & 1 . & $\begin{array}{l}0-6 \\
6-12\end{array}$ & $\begin{array}{r}95.7 \\
105.1\end{array}$ & $\begin{array}{l}17.0 \\
16.6\end{array}$ & $\begin{array}{l}116 \\
153\end{array}$ & $\overline{0.60}$ & $\overline{92}$ & 0.3 & 0.42 & 0.0 & 0.36 & & & & & & & & & \\
\hline $20 \mathrm{~F}$ & 1 & $\begin{array}{l}0-6 \\
6-12\end{array}$ & $\begin{array}{l}94.0 \\
99.8\end{array}$ & $\begin{array}{l}15.6 \\
16.3\end{array}$ & $\begin{array}{l}207 \\
212\end{array}$ & $\overline{0.67}$ & $\overline{146}$ & 1.0 & 0.38 & 0.0 & 0.18 & & & & & & & & & \\
\hline $2 L A$ & 1 & $\begin{array}{l}0-6 \\
6-12\end{array}$ & $\begin{array}{r}92.7 \\
100.9\end{array}$ & $\begin{array}{l}22.6 \\
20.1\end{array}$ & $\begin{array}{r}93 \\
119\end{array}$ & $\overline{0.54}$ & $\overline{64}$ & 0.8 & 0.32 & 1.2 & 0.32 & & & & & & & & & \\
\hline $21 \mathrm{~B}$ & 1 & $\begin{array}{l}0-6 \\
6-12\end{array}$ & $\begin{array}{l}90.1 \\
94.6\end{array}$ & $\begin{array}{l}30.4 \\
27.4\end{array}$ & $\begin{array}{l}54 \\
90\end{array}$ & 0.53 & $\overline{48}$ & 0.9 & 0.09 & 0.5 & 0.05 & $\begin{array}{l}90.1 \\
94.6\end{array}$ & $\begin{array}{l}30.4 \\
27.4\end{array}$ & $\begin{array}{l}54 \\
90\end{array}$ & 0.53 & $-\overline{4 B}$ & 0.9 & 0.09 & 0.50 .05 & +5.0 \\
\hline $21 \mathrm{c}$ & 1 & $\begin{array}{l}0-6 \\
6-12\end{array}$ & $\begin{array}{l}102.6 \\
101.3\end{array}$ & $\begin{array}{l}16.6 \\
21.5\end{array}$ & $\begin{array}{r}81 \\
117\end{array}$ & $\overline{0.35}$ & $\overline{41}$ & 1.0 & 0.18 & 0.1 & 0.18 & $\begin{array}{l}102.6 \\
101.3\end{array}$ & $\begin{array}{l}16.6 \\
21.5\end{array}$ & $\begin{array}{r}81 \\
117\end{array}$ & $\overline{0.35}$ & $\overline{41}$ & 1.0 & 0.18 & 0.10 .18 & +0.5 \\
\hline 210 & 1 & $\begin{array}{l}0-6 \\
6-12\end{array}$ & $\begin{array}{l}88.0 \\
96.3\end{array}$ & $\begin{array}{l}33.1 \\
25.6\end{array}$ & $\begin{array}{r}92 \\
123\end{array}$ & $\overline{0.37}$ & $\overline{46}$ & 1.5 & 0.16 & 0.6 & 0.24 & $\begin{array}{l}88.0 \\
96.3\end{array}$ & $\begin{array}{l}33.1 \\
25.6\end{array}$ & $\begin{array}{r}92 \\
123\end{array}$ & $0 .-\overline{37}$ & $\overline{46}$ & 1.5 & 0.16 & $0.6 \quad 0.14$ & +2.0 \\
\hline $21 E$ & 1 & $\begin{array}{l}0-6 \\
6-12\end{array}$ & $\begin{array}{r}91.9 \\
100.6\end{array}$ & $\begin{array}{l}28.0 \\
21.9\end{array}$ & $\begin{array}{r}59 \\
142\end{array}$ & $\overline{0.36}$ & $\overline{51}$ & 1.5 & 0.34 & 0.3 & 0.30 & $\begin{array}{r}91.9 \\
100.6\end{array}$ & $\begin{array}{l}28.0 \\
21.9\end{array}$ & $\begin{array}{r}59 \\
142\end{array}$ & $\overline{0.36}$ & 51 & 1.5 & 0.34 & 0.30 .30 & 0.0 \\
\hline $21 F$ & 1 & $\begin{array}{l}0-6 \\
6-12\end{array}$ & $\begin{array}{l}98.4 \\
98.7\end{array}$ & $\begin{array}{l}20.0 \\
18.8\end{array}$ & $\begin{array}{l}159 \\
308\end{array}$ & $\overline{0.24}$ & $\overline{91}$ & -- & - & -- & -- & $\begin{array}{l}98.4 \\
98.7\end{array}$ & $\begin{array}{l}20.0 \\
18.8\end{array}$ & $\begin{array}{l}159 \\
308\end{array}$ & 0.24 & $\overrightarrow{91}$ & -- & -- & -- & +6.0 \\
\hline $22 \mathrm{~A}$ & 1 & $\begin{array}{l}0-6 \\
6-12\end{array}$ & $\begin{array}{l}91.2 \\
97.7\end{array}$ & $\begin{array}{l}25.9 \\
22.8\end{array}$ & $\begin{array}{r}56 \\
111\end{array}$ & $\overline{0.53}$ & $\overline{59}$ & 1.5 & 0.28 & 0.5 & 0.14 & & & & & & & & & \\
\hline $22 B$ & 1 & $\begin{array}{l}0-6 \\
6-12\end{array}$ & $\begin{array}{l}76.9 \\
67.5\end{array}$ & $\begin{array}{l}39.4 \\
33.4\end{array}$ & $\begin{array}{l}18 \\
48\end{array}$ & $\overline{0.54}$ & $\overline{26}$ & 1.0 & 0.18 & 0.4 & 0.18 & $\begin{array}{l}76.9 \\
87.5\end{array}$ & $\begin{array}{l}39.4 \\
33.4\end{array}$ & $\begin{array}{l}18 \\
48\end{array}$ & 0.54 & $\overline{26}$ & 1.0 & 0.18 & 0.40 .18 & +6.0 \\
\hline $22 \mathrm{C}$ & 1 & $\begin{array}{l}0-6 \\
6-12\end{array}$ & $\begin{array}{l}87.1 \\
98.5\end{array}$ & $\begin{array}{l}26.2 \\
21.5\end{array}$ & $\begin{array}{l}57 \\
84\end{array}$ & $\overline{0.73}$ & $\overline{61}$ & 1.7 & 0.38 & 0.7 & 0.20 & & & & & & & & & \\
\hline 22D & 1 & $\begin{array}{l}0-6 \\
6-12\end{array}$ & $\begin{array}{l}95.2 \\
97.4\end{array}$ & $\begin{array}{l}24.7 \\
24.3\end{array}$ & $\begin{array}{l}181 \\
226\end{array}$ & $0 .-\overline{29}$ & $\overrightarrow{66}$ & 0.8 & 0.14 & 1.0 & 0.09 & $\begin{array}{l}95.2 \\
97.4\end{array}$ & $\begin{array}{l}24.7 \\
24.3\end{array}$ & $\begin{array}{l}181 \\
226\end{array}$ & 0.29 & $\overline{66}$ & 0.8 & 0.14 & 1.00 .09 & +2.0 \\
\hline $22 \mathrm{E}$ & 1 & $\begin{array}{l}0-6 \\
6-12\end{array}$ & $\begin{array}{l}92.8 \\
94.2\end{array}$ & $\begin{array}{l}26.5 \\
24.3\end{array}$ & $\begin{array}{l}214 \\
313\end{array}$ & $\overline{0.25}$ & $\overline{78}$ & 0.7 & 0.30 & 0.2 & 0.03 & & & & & & & & & \\
\hline $23 \mathrm{~A}$ & 1 & $\begin{array}{l}0-6 \\
6-12\end{array}$ & $\begin{array}{l}81.8 \\
87.5\end{array}$ & $\begin{array}{l}37.3 \\
33.2\end{array}$ & $\begin{array}{l}101 \\
157\end{array}$ & $0 . \overline{0.43}$ & -78 & 0.8 & 0.53 & 0.5 & 0.18 & $\begin{array}{l}81.8 \\
87.5\end{array}$ & $\begin{array}{l}37.3 \\
33.2\end{array}$ & $\begin{array}{l}101 \\
157\end{array}$ & $\overline{0.43}$ & $\overline{68}$ & 0.8 & 0.53 & 0.50 .18 & 0.0 \\
\hline $23 B$ & 1 & $\begin{array}{l}0-6 \\
6-12\end{array}$ & $\ddot{-}$ & $\begin{array}{l}34.3 \\
37.4\end{array}$ & $\begin{array}{l}137 \\
220\end{array}$ & $\begin{array}{l}-- \\
--\end{array}$ & -- & 0.7 & 0.42 & 1.2 & 0.09 & & & & & & & & & \\
\hline $23 \mathrm{C}$ & 1 & $\begin{array}{l}0-6 \\
6-12\end{array}$ & $\overline{--}$ & $\begin{array}{l}34.9 \\
31.7\end{array}$ & $\begin{array}{l}150 \\
219\end{array}$ & $=$ & $=$ & 1.8 & 0.27 & 1.2 & 0.03 & & & & & & & & & \\
\hline $23 D$ & 1 & $\begin{array}{l}0-6 \\
6-12\end{array}$ & $\overline{-}$ & $\begin{array}{l}22.9 \\
28.6\end{array}$ & $\begin{array}{l}421+ \\
672+\end{array}$ & -- & $\overline{-}$ & 0.3 & 0.45 & 0.0 & 0.18 & & & & & & & & & \\
\hline $23 \mathrm{~B}$ & 1 & $\begin{array}{l}0-6 \\
6-12\end{array}$ & $\begin{array}{r}96.4 \\
105.2\end{array}$ & $\begin{array}{l}21.6 \\
14.8\end{array}$ & $\begin{array}{l}236 \\
182\end{array}$ & $\overline{0.18}$ & $\overline{33}$ & 0.5 & 0.32 & 0.5 & 0.25 & & & & & & & & & \\
\hline $23 F$ & 1 & $\begin{array}{l}0-6 \\
6-12\end{array}$ & $\begin{array}{l}98.3 \\
98.5\end{array}$ & $\begin{array}{l}15.1 \\
21.5\end{array}$ & $\begin{array}{r}95 \\
108\end{array}$ & $\overline{0.18}$ & $\overline{19}$ & 0.0 & 0.40 & 0.2 & 0.30 & & & & & & & & & \\
\hline $23 \mathrm{G}$ & 1 & $\begin{array}{l}0-6 \\
6-12\end{array}$ & $\begin{array}{l}104.3 \\
110.0\end{array}$ & $\begin{array}{l}15.4 \\
12.4\end{array}$ & $\begin{array}{r}82 \\
295\end{array}$ & 0.25 & $\overline{74}$ & 0.7 & 0.34 & 0.4 & 0.23 & $\begin{array}{l}104.3 \\
110.0\end{array}$ & $\begin{array}{l}15.4 \\
12.4\end{array}$ & $\begin{array}{r}82 \\
295\end{array}$ & 0.25 & $\overline{74}$ & 0.7 & 0.34 & 0.40 .23 & +4.0 \\
\hline $24 A$ & 1 & $\begin{array}{l}0-6 \\
6-12\end{array}$ & $\begin{array}{l}86.4 \\
88.5\end{array}$ & $\begin{array}{l}28.4 \\
28.7\end{array}$ & $\begin{array}{r}97 \\
168\end{array}$ & 0.54 & $\overrightarrow{9 i}$ & 1.8 & 0.16 & 0.8 & 0.14 & $\begin{array}{l}86.4 \\
88.5\end{array}$ & $\begin{array}{l}28.4 \\
28.7\end{array}$ & $\begin{array}{r}97 \\
168\end{array}$ & 0.54 & $\overline{91}$ & -- & -- & - & 4.0 \\
\hline $24 B$ & 1 & $\begin{array}{l}0-6 \\
6-12\end{array}$ & $\begin{array}{l}89.8 \\
--\end{array}$ & $\begin{array}{l}23.6 \\
15.0\end{array}$ & $\begin{array}{l}290+ \\
750+\end{array}$ & -- & 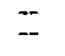 & 2.0 & 0.16 & 0.0 & 0.18 & & & & & & & & & \\
\hline $24 \mathrm{C}$ & 1 & $\begin{array}{l}0-6 \\
6-12\end{array}$ & $\begin{array}{l}85.6 \\
95.0\end{array}$ & $\begin{array}{l}31.1 \\
22.6\end{array}$ & $\begin{array}{r}87 \\
130\end{array}$ & $\overline{0.69}$ & 90 & 2.1 & 0.22 & 0.1 & 0.25 & & & & & & & & & \\
\hline $24 \mathrm{D}$ & 1 & $\begin{array}{l}0-6 \\
6-12\end{array}$ & $\begin{array}{l}83.9 \\
84.4\end{array}$ & $\begin{array}{l}15.6 \\
20.2\end{array}$ & $\begin{array}{c}93 \\
252+\end{array}$ & $\overline{1.60}$ & $\overline{403+}$ & 0.5 & 0.32 & 0.2 & 0.28 & & & & & & & & & \\
\hline $25 \mathrm{~A}$ & 1 & $\begin{array}{l}0-6 \\
6-12\end{array}$ & $\begin{array}{l}77.6 \\
79.6\end{array}$ & $\begin{array}{l}29.0 \\
14.7\end{array}$ & $\begin{array}{r}71 \\
250\end{array}$ & $\overline{0.93}$ & $\overline{140}$ & 1.3 & 0.45 & 0.0 & 0.30 & & & & & & & & & \\
\hline
\end{tabular}


Table A2 (Continued)

\begin{tabular}{|c|c|c|c|c|c|c|c|c|c|c|c|c|c|c|c|c|c|c|c|}
\hline \multirow[b]{2}{*}{$\begin{array}{l}\text { Site } \\
\text { llo. } \\
\end{array}$} & & \multirow[b]{2}{*}{$\begin{array}{l}\text { Topog- } \\
\text { raphy } \\
\text { Class } \\
\end{array}$} & \multicolumn{3}{|c|}{ Ection A. Site Da } & \multirow[b]{2}{*}{ Land Use } & \multirow[b]{2}{*}{$\begin{array}{l}\text { Depth } \\
\text { of } \\
\text { Layer } \\
\text { in. } \\
\end{array}$} & \multicolumn{9}{|c|}{ Section B. Soil Data } & \multirow[b]{2}{*}{$\begin{array}{l}\text { Or- } \\
\text { ganic } \\
\text { Con- } \\
\text { tent } \\
\text { tent } \\
\text { pon }\end{array}$} & \multirow[b]{2}{*}{$\begin{array}{l}\text { ic Spe- } \\
\text { ciric } \\
\text { Grav- } \\
\text { Gity } \\
\end{array}$} \\
\hline & $\begin{array}{c}\text { Map } \\
\text { Sheet } \\
\end{array}$ & $\begin{array}{c}\text { Grid } \\
\text { Cuor- } \\
\text { di- } \\
\text { nates } \\
\end{array}$ & & \multicolumn{3}{|r|}{ Vegetation } & & & & $\begin{array}{l}\text { US } \\
\text { exture } \\
\text { y wit, } \\
\text { Silt }\end{array}$ & 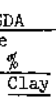 & Type & $\begin{array}{l}\text { By wt } \\
\% \\
\text { Fines } \\
\end{array}$ & 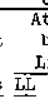 & 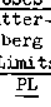 & $\underline{\underline{\text { PI }}}$ & Type & & \\
\hline $25 B$ & $4866 \mathrm{I}$ & 891572 & Low & $\begin{array}{l}\text { Terrace } \\
\text { slope }\end{array}$ & 2 & $\begin{array}{l}\text { Tall scrub } \\
\text { savanna }\end{array}$ & $\begin{array}{l}\text { App1e } \\
\text { orchard }\end{array}$ & $\begin{array}{l}0-6 \\
6-12\end{array}$ & $\overline{45}$ & $\overline{33}$ & $\overline{22}$ & $\overline{\mathrm{L}}$ & 26 & $\overline{26}$ & $\overline{16}$ & - & $\overline{\mathrm{CL}}$ & -- & $2 .-6$ \\
\hline $25 \mathrm{C}$ & 48661 & 869571 & High & Terrace $f 1 a t$ & $=0$ & Savanna & Grazed & $\begin{array}{l}0-6 \\
6-12\end{array}$ & $\overline{75}$ & $\overline{18}$ & $\overline{7}$ & $\overline{\mathrm{SL}}$ & $\overline{32}$ & $=$ & -- & $\overline{\mathrm{NP}}$ & $\overline{S M}$ & 0.86 & -- \\
\hline $26 \mathrm{~A}$ & $4767 I$ & 948978 & Low & $\begin{array}{l}\text { BottomIand } \\
\text { f lat }\end{array}$ & 0 & $\begin{array}{l}\text { Tal1 scrub } \\
\text { savanna }\end{array}$ & $\begin{array}{l}\text { Cu1tivated } \\
\text { (rice) }\end{array}$ & $\begin{array}{l}0-6 \\
6-12\end{array}$ & $\overline{55}$ & $\overline{17}$ & $\overline{28}$ & $\overline{\mathrm{sCL}}$ & $\overline{51}$ & $\tilde{25}$ & $\overline{12}$ & $\overline{13}$ & $\overline{\mathrm{CL}}$ & 1.05 & $=$ \\
\hline $26 \mathrm{~B}$ & $4767 \mathrm{I}$ & 948978 & Low & Terrace flat & 0 & $\begin{array}{l}\text { Tal1 scrub } \\
\text { savanna }\end{array}$ & Undisturbed & $\begin{array}{l}0-6 \\
6-12\end{array}$ & $\overline{43}$ & $\overline{38}$ & $\overline{19}$ & $\bar{L}$ & $-\overline{62}$ & $\overline{28}$ & $\overline{18}$ & $\overline{10}$ & $\overline{\mathrm{CL}}$ & $3 .-96$ & $=-$ \\
\hline $26 \mathrm{C}$ & 47671 & 947978 & H1gh & Lower slope & 9 & $\begin{array}{l}\text { Tall-grass } \\
\text { prairie }\end{array}$ & $\begin{array}{l}\text { Banana } \\
\text { orchard }\end{array}$ & $\begin{array}{l}0-6 \\
6-12\end{array}$ & $\ddot{76}$ & $\overline{16}$ & $-\overline{8}$ & $\overline{\mathrm{SL}}$ & $\overline{28}$ & $\overline{--}$ & $\begin{array}{l}-- \\
--\end{array}$ & $\overline{\mathrm{NP}}$ & $\overline{S M}$ & $\overline{1.10}$ & 2.64 \\
\hline 260 & $4767 \mathrm{I}$ & 947978 & High & Upper slope & 9 & $\begin{array}{l}\text { Tall scrub } \\
\text { savanna }\end{array}$ & $\begin{array}{c}\text { Cultivated } \\
\text { (peanuts) }\end{array}$ & $\begin{array}{l}0-6 \\
6-12\end{array}$ & $\overline{77}$ & $\overline{19}$ & $-\overline{4}$ & $\overline{\text { LS }}$ & $\overline{28}$ & $\begin{array}{l}-- \\
--\end{array}$ & -- & $\overrightarrow{\mathrm{NP}}$ & $\overline{S M}$ & $\overline{0.62}$ & $=$ \\
\hline $26 \mathrm{E}$ & 47671 & 947978 & High & Up 1 and $\mathrm{flat}$ & 0 & $\begin{array}{l}\text { Ta11 scrub } \\
\text { wood1and }\end{array}$ & Undisturbed & $\begin{array}{l}0-6 \\
6-12\end{array}$ & $\overline{74}$ & $\overline{15}$ & $\overline{11}$ & $\overline{\mathrm{SL}}$ & $\overline{31}$ & -- & $\begin{array}{l}-- \\
--\end{array}$ & $\overline{\mathrm{NP}}$ & $\overline{S M}$ & -- & -- \\
\hline $27 \mathrm{~A}$ & $47671 \mathrm{I}$ & 962791 & Low & Terrace f1at & 0 & $\begin{array}{l}\text { Short-grass } \\
\text { prairie }\end{array}$ & Grazed & $\begin{array}{l}0-6 \\
6-12\end{array}$ & $\overline{58}$ & $\overline{25}$ & $\overline{17}$ & $\overline{\mathrm{sL}}$ & $-\overline{47}$ & $\overline{19}$ & $--\overline{13}$ & $\overline{6}$ & -- & $-\overline{0.78}$ & $\because$ \\
\hline $27 \mathrm{~B}$ & 476711 & 961792 & Low & Lower slope & 1 & $\begin{array}{l}\text { Short-grass } \\
\text { prairfe }\end{array}$ & Grazed & $\begin{array}{l}0-6 \\
6-12\end{array}$ & $\overline{55}$ & $\overline{28}$ & $\overline{17}$ & $\overline{\mathrm{SL}}$ & 52 & $\overline{22}$ & $\overline{14}$ & $\overline{8}$ & $\overline{C L}$ & $\overline{0.78}$ & $\overline{2.62}$ \\
\hline $27 \mathrm{C}$ & $4767 \mathrm{II}$ & 959793 & Low & Lower slope & 1 & $\begin{array}{l}\text { Short-grass } \\
\text { praitie }\end{array}$ & Grazed & $\begin{array}{l}0-6 \\
6-12\end{array}$ & $\overline{63}$ & $\overline{25}$ & $\overline{12}$ & $\overline{s L}$ & -- & $\overline{17}$ & $\overline{14}$ & $-\overline{3}$ & $\overline{\text { SM }}$ & $1 .-40$ & -- \\
\hline $27 \mathrm{D}$ & $4767 I I$ & 959794 & Low & $\begin{array}{l}\text { Natural } \\
\text { levee }\end{array}$ & 0 & Savanna & $\begin{array}{l}\text { Banana } \\
\text { orchard }\end{array}$ & $\begin{array}{l}0-6 \\
6-12\end{array}$ & $\overline{57}$ & $\overline{24}$ & $\overline{19}$ & $\overline{\mathrm{SL}}$ & $\overline{49}$ & $\overline{22}$ & $\overline{15}$ & $\overline{7}$ & $\overline{S M-S C}$ & $=1.40$ & $\overline{--}$ \\
\hline & & & & & & & athabur1 Area & & & & & & & & & & & & \\
\hline $28 \mathrm{~A}$ & $5448 \mathrm{IV}$ & 889951 & Low & $\begin{array}{l}\text { Terrace } \\
\text { slope }\end{array}$ & 2 & $\begin{array}{l}\text { Short-gra } \\
\text { prairie }\end{array}$ & Grazed & $\begin{array}{l}0-6 \\
6-12\end{array}$ & $\overline{52}$ & $\overline{22}$ & $\overline{26}$ & $\overline{S C L}$ & $\overline{54}$ & $\overline{32}$ & $\begin{array}{l}-- \\
14\end{array}$ & $\overline{18}$ & $\overline{c L}$ & $--\overline{1.72}$ & 2.66 \\
\hline $28 \mathrm{~B}$ & $5448 \mathrm{IV}$ & 889952 & Low & $\begin{array}{l}\text { Terrace } \\
\text { slope }\end{array}$ & 2 & $\begin{array}{l}\text { Short-grass } \\
\text { prairie }\end{array}$ & Grazed & $\begin{array}{l}0-6 \\
6-12\end{array}$ & $\overline{53}$ & $\overline{24}$ & $\overline{23}$ & $\overline{S C L}$ & $\overline{52}$ & $\overline{24}$ & $\overline{15}$ & $\overline{9}$ & $\overline{\mathrm{CL}}$ & $\overline{0.78}$ & $\overline{--}$ \\
\hline $28 \mathrm{C}$ & $54481 \mathrm{~V}$ & 889952 & Low & $\begin{array}{l}\text { Terrace } \\
\text { slope }\end{array}$ & 2 & $\begin{array}{l}\text { Short-grass } \\
\text { prairie }\end{array}$ & Grazed & $\begin{array}{l}0-6 \\
6-12\end{array}$ & $\overline{53}$ & $\overline{26}$ & $\overline{21}$ & $\overline{\mathrm{SCL}}$ & $\overline{51}$ & 25 & $\overline{16}$ & $\overline{9}$ & $\overline{\mathrm{CL}}$ & $\overline{--}$ & $\overline{--}$ \\
\hline $28 \mathrm{D}$ & $5448 \mathrm{IV}$ & 889953 & Low & Terrace $\mathrm{f}$ lat & 0 & $\begin{array}{l}\text { Short-grass } \\
\text { prairie }\end{array}$ & Grazed & $\begin{array}{l}0-6 \\
6-12\end{array}$ & $\overline{51}$ & $\overline{24}$ & $\overline{25}$ & $\overline{S C L}$ & $\overline{58}$ & $\overline{2 B}$ & $\overline{17}$ & $\overline{11}$ & $\overline{\mathrm{CL}}$ & $\overline{1.88}$ & 2.66 \\
\hline $29 \mathrm{~A}$ & $5448 \mathrm{IV}$ & 840933 & Low & $\begin{array}{l}\text { Bot tomland } \\
\text { flat }\end{array}$ & 0 & $\begin{array}{l}\text { Short-grass } \\
\text { prairie }\end{array}$ & $\begin{array}{l}\text { Cultivated } \\
\text { (grazed) }\end{array}$ & $\begin{array}{l}0-6 \\
6-12\end{array}$ & $\overline{37}$ & $\overline{43}$ & 20 & $\overline{\mathrm{L}}$ & $\overline{67}$ & $-\overline{44}$ & $\overline{24}$ & $\overline{20}$ & $\overline{\mathrm{CL}}$ & $5 . \overline{74}$ & $2 .-\overline{66}$ \\
\hline $29 B$ & $5448 \mathrm{IV}$ & 838932 & Low & $\begin{array}{l}\text { Bottomland } \\
\text { flat }\end{array}$ & 0 & $\begin{array}{l}\text { Tall-grass } \\
\text { prairie }\end{array}$ & $\begin{array}{l}\text { Cultivated } \\
\text { (idle) }\end{array}$ & $\begin{array}{l}0-6 \\
6-12\end{array}$ & $\overline{34}$ & 45 & $\overline{21}$ & $\bar{L}$ & $\overrightarrow{78}$ & $-\overline{88}$ & $\overline{33}$ & -2 & $\overline{\mathrm{CH}} \quad 1$ & 15.00 & $2 .-46$ \\
\hline $29 \mathrm{C}$ & $54481 \mathrm{~V}$ & 841934 & Low & $\begin{array}{l}\text { Bot tomland } \\
\text { f lat }\end{array}$ & 0 & $\begin{array}{l}\text { Short-grass } \\
\text { prairie }\end{array}$ & Grazed & $\begin{array}{l}0-6 \\
6-12\end{array}$ & $\overline{27}$ & -- & $\overline{27}$ & $\overline{\mathrm{L}}$ & $\overline{78}$ & 34 & $\overline{17}$ & $\overline{17}$ & $\overline{\mathrm{CL}}$ & 2.90 & -- \\
\hline $30 \mathrm{~A}$ & $5448 \mathrm{IV}$ & 759003 & Low & $\begin{array}{l}\text { Natural } \\
\text { levee }\end{array}$ & 0 & $\begin{array}{l}\text { Tal1-grass } \\
\text { prairie }\end{array}$ & $\begin{array}{l}\text { Cultivated } \\
\text { (Idle) }\end{array}$ & $\begin{array}{l}0-6 \\
6-12\end{array}$ & $\overline{54}$ & $\overline{19}$ & $\overline{27}$ & $\overline{\mathrm{SCL}}$ & $\overrightarrow{58}$ & $\overline{28}$ & $\overline{16}$ & $\overline{--}$ & $\overline{\mathrm{CL}}$ & 3.10 & -- \\
\hline 30B & $5448 \mathrm{IV}$ & 759004 & Low & $\begin{array}{l}\text { Terrace } \\
\text { slope }\end{array}$ & 2 & $\begin{array}{l}\text { Tal1 } \\
\text { wood1 }\end{array}$ & $\begin{array}{l}\text { Cul tivated } \\
\text { (pepper) }\end{array}$ & $\begin{array}{l}0-6 \\
6-12\end{array}$ & $\overline{62}$ & $\overline{17}$ & $\overline{21}$ & $\overline{\mathrm{SCL}}$ & $\overline{48}$ & $-\overline{23}$ & $\overline{15}$ & $\overline{8}$ & $\overline{\mathrm{sc}}$ & $\overline{--}$ & $\overline{-}$ \\
\hline $30 \mathrm{C}$ & $5448 \mathrm{IV}$ & 758005 & Low & $\begin{array}{l}\text { Terrace } \\
\text { slope }\end{array}$ & 2. & Hoodland & Undisturbed & $\begin{array}{l}0-6 \\
6-12\end{array}$ & $\overline{68}$ & $\overline{12}$ & $\overline{20}$ & $\overline{\text { SL }}$ & $\overline{41}$ & $\overline{17}$ & $\overline{12}$ & $\overline{5}$ & $\overline{S M-S C}$ & 1.40 & 2.64 \\
\hline 300 & $5448 \mathrm{IV}$ & 758006 & Low & Terrace flat & 0 & Woodland & Undisturbed & $\begin{array}{l}0-6 \\
6-12\end{array}$ & $\overline{62}$ & $\overline{16}$ & $\overline{22}$ & $\overline{\mathrm{SCL}}$ & $\overline{51}$ & $\overline{23}$ & $\overline{14}$ & -- & $\overrightarrow{C L}$ & $\overline{1.72}$ & $=$ \\
\hline $31 \mathrm{~A}$ & $53491 \mathrm{II}$ & 213078 & Low & $\begin{array}{l}\text { Natura } 1 \\
\text { levee }\end{array}$ & 5 & $\begin{array}{l}\text { Ta11 scru } \\
\text { woodland }\end{array}$ & Jndisturbed & $\begin{array}{l}0-6 \\
6-12\end{array}$ & $\overline{41}$ & 37 & $\overline{22}$ & $\bar{L}$ & $-\overline{76}$ & $-\overline{41}$ & $\overline{25}$ & $\overline{16}$ & $\overline{\mathrm{CL}}$ & $1 .-$ & 2.66 \\
\hline $31 \mathrm{~B}$ & $5349 \mathrm{II}$ & 213078 & Low & $\begin{array}{l}\text { Bottomland } \\
\text { flat }\end{array}$ & 0 & $\begin{array}{l}\text { Short-grass } \\
\text { prairie }\end{array}$ & $\begin{array}{l}\text { Culti } \\
\text { (1d1 }\end{array}$ & -6 & $\overline{11}$ & $\overline{57}$ & $\overline{32}$ & $\overline{S i C L}^{--}$ & $\overline{93}$ & $\overline{65}$ & $\overline{33}$ & $\overline{32}$ & $\overrightarrow{\mathrm{MH}}$ & 2.30 & $\overline{--}$ \\
\hline $31 \mathrm{C}$ & 534911 & 212078 & Low & $\begin{array}{l}\text { Bottomland } \\
\text { depression }\end{array}$ & 0 & $\begin{array}{l}\text { Tal1-grass } \\
\text { pratrie }\end{array}$ & Undisturbed & $\begin{array}{l}0-6 \\
6-12\end{array}$ & $\overline{17}$ & 53 & $\overline{30}$ & $\overline{\text { SiCL }}$ & $\overline{94}$ & $\overline{s 5}$ & $\overline{28}$ & $\overline{27}$ & $\overline{\mathrm{CH}} \quad 1$ & $\overline{1.90}$ & $=$ \\
\hline $32 \mathrm{~A}$ & $5349 \mathrm{II}$ & 141101 & Low & Terrace flat & 0 & $\begin{array}{l}\text { Tall-grass } \\
\text { prairle }\end{array}$ & Undisturbed & $\begin{array}{l}0-6 \\
6-12\end{array}$ & $\tilde{68}$ & $\overline{24}$ & $-\overline{8}$ & $\overline{\mathrm{SL}}$ & $\overline{49}$ & -- & $\overline{--}$ & $-\overline{\mathrm{NP}}$ & $\overrightarrow{S M}$ & 0.86 & -- \\
\hline 32B & $5349 \mathrm{II}$ & 141101 & Low & Lower slope & 8 & $\begin{array}{l}\text { Tall-grass } \\
\text { prairfe }\end{array}$ & Dndisturbed & $\begin{array}{l}0-6 \\
6-12\end{array}$ & $\overline{67}$ & $\overrightarrow{22}$ & $\overline{11}$ & $\overline{S L}$ & $\overline{49}$ & $\overline{12}$ & -- & $\overline{\mathrm{NP}}$ & $\overline{\mathrm{SM}}$ & -- & 2.64 \\
\hline $32 \mathrm{C}$ & 534911 & 141102 & Low & Upper slope 1 & 10 & $\begin{array}{l}\text { Low scr } \\
\text { Savanna }\end{array}$ & $\begin{array}{l}\text { Cultivated } \\
\text { (rice) }\end{array}$ & $\begin{array}{l}0-6 \\
6-12\end{array}$ & $\overline{47}$ & $\overline{35}$ & $\overline{18}$ & $\bar{L}$ & $\overline{61}$ & $\overline{27}$ & $\overline{--}$ & $\overline{11}$ & $\overline{\mathrm{CL}} \quad 0$ & 0.86 & -- \\
\hline 32D & 534911 & 141102 & Low & Upper slope 1 & 11 & $\begin{array}{l}\text { Low scrub } \\
\text { savanna }\end{array}$ & (rice) & $\begin{array}{l}0-6 \\
6-12\end{array}$ & $\overline{52}$ & $\overline{26}$ & $\overline{z 2}$ & $\overline{\mathrm{SCL}}$ & $\overline{61}$ & $\overline{24}$ & $\overline{--}$ & -- & $\overline{\mathrm{CL}} \quad 0$ & $\overline{0.86}$ & 2.68 \\
\hline $33 \mathrm{~A}$ & $5448 \mathrm{TV}$ & 759001 & Low & $\begin{array}{l}\text { Bottomland } \\
\text { depression }\end{array}$ & 0 & Woodland & plantation & $6-12$ & 12 & $\overline{57}$ & $\overline{3 I}$ & $\overline{S 1 C L}$ & $\overline{92}$ & $\overline{53}$ & $\overline{33}$ & $\overline{20}$ & M- & 2.50 & $\begin{array}{l}-- \\
2.64\end{array}$ \\
\hline
\end{tabular}




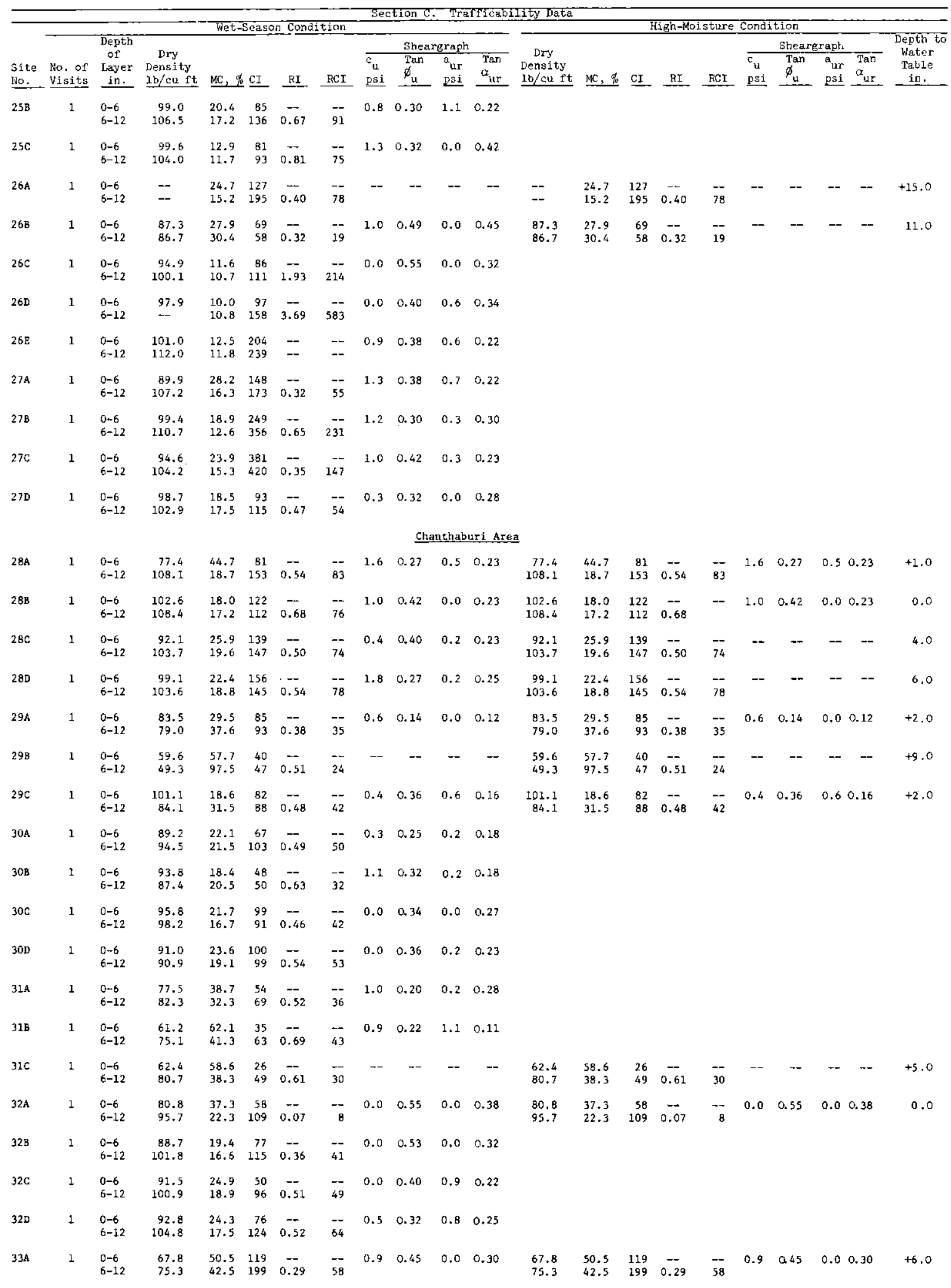




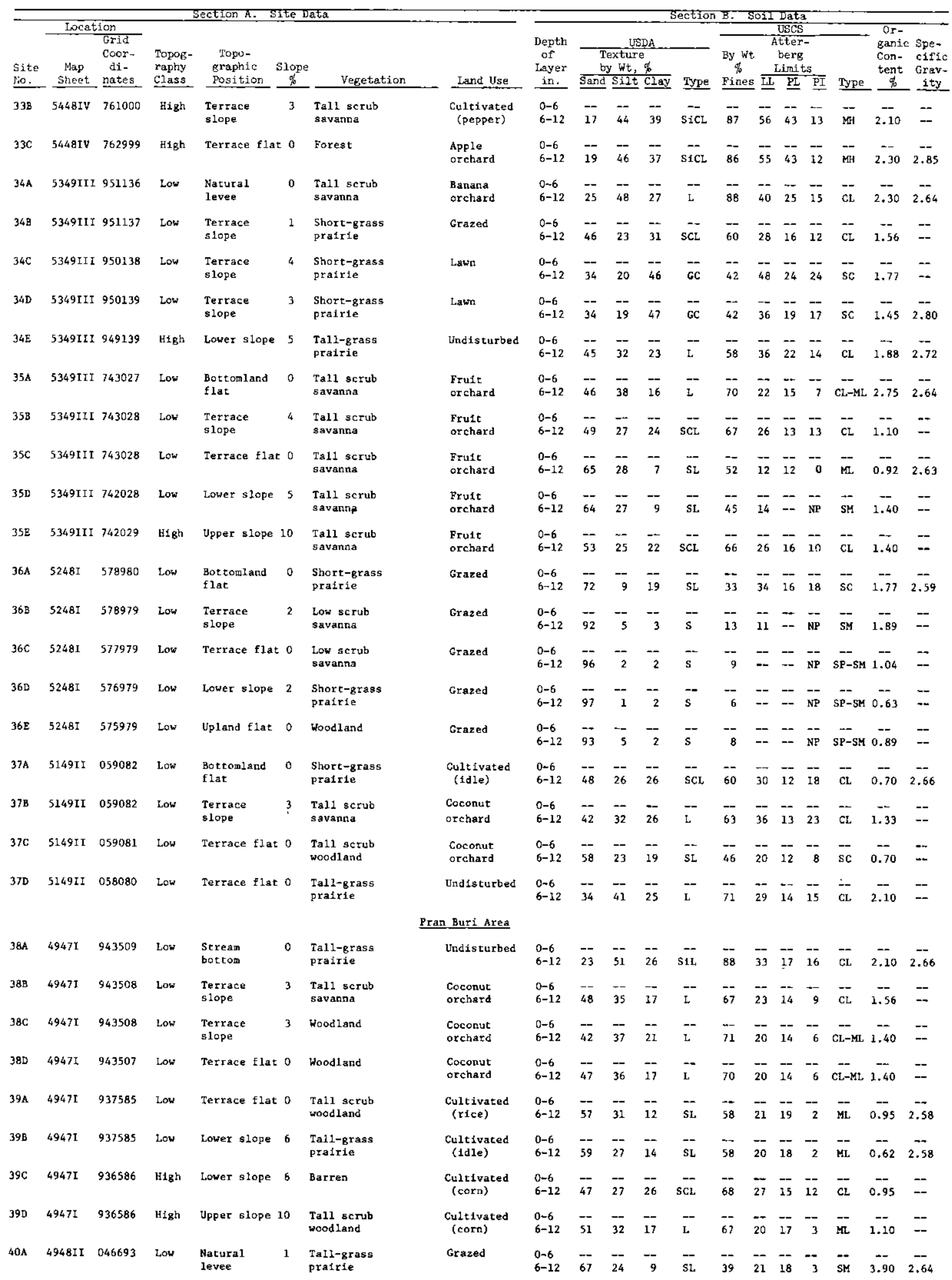




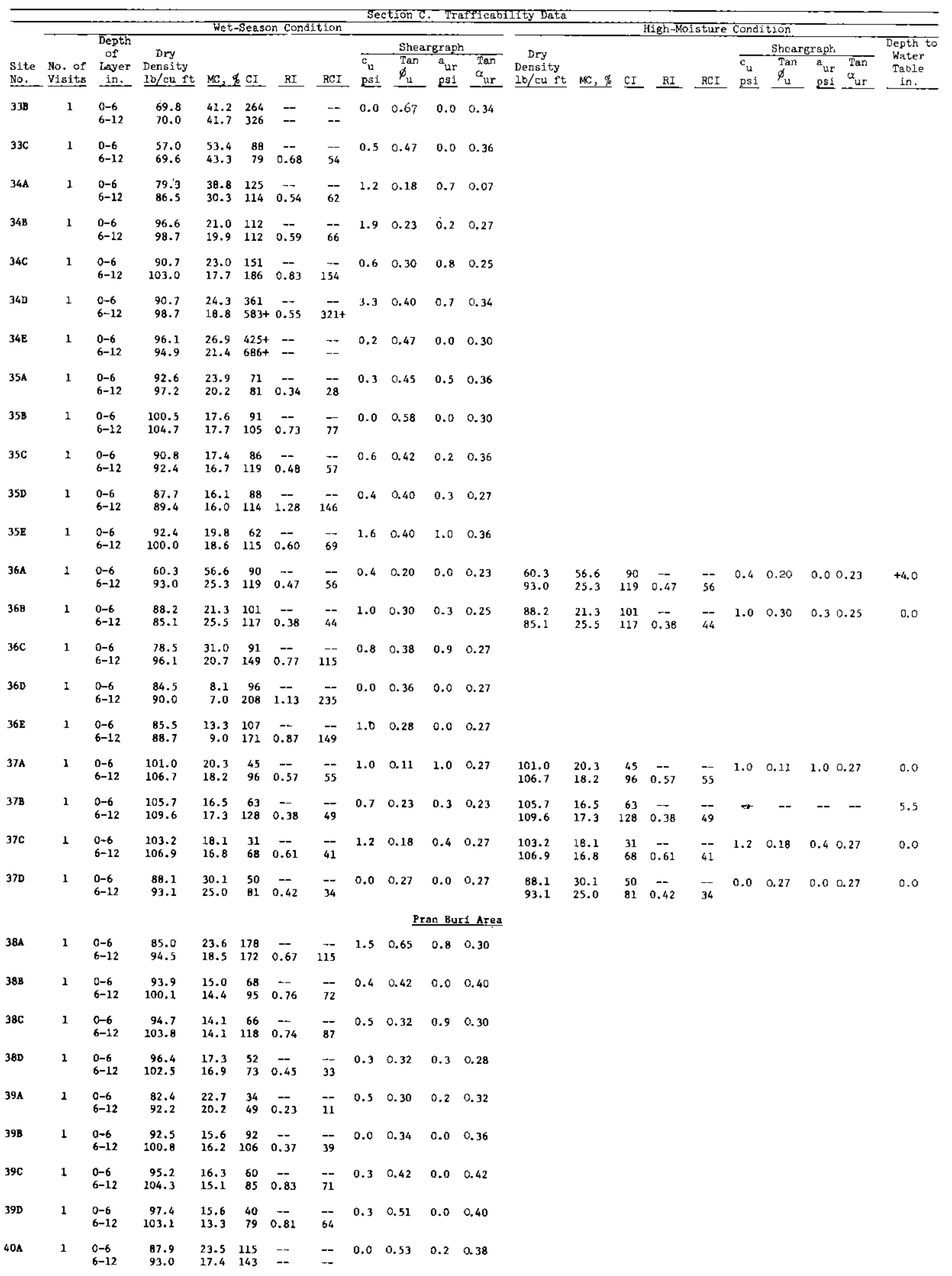


Table A2 (Continued)

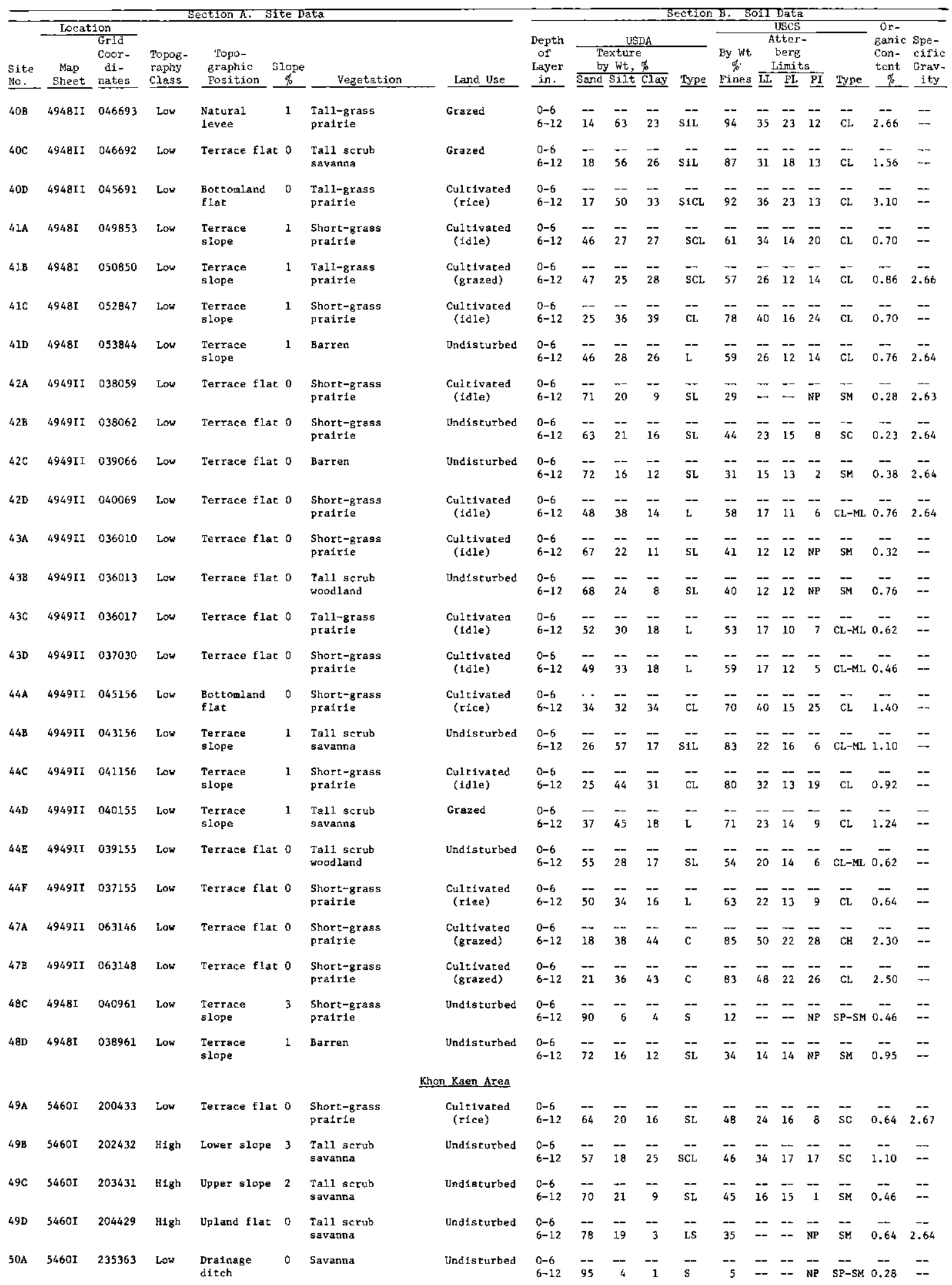




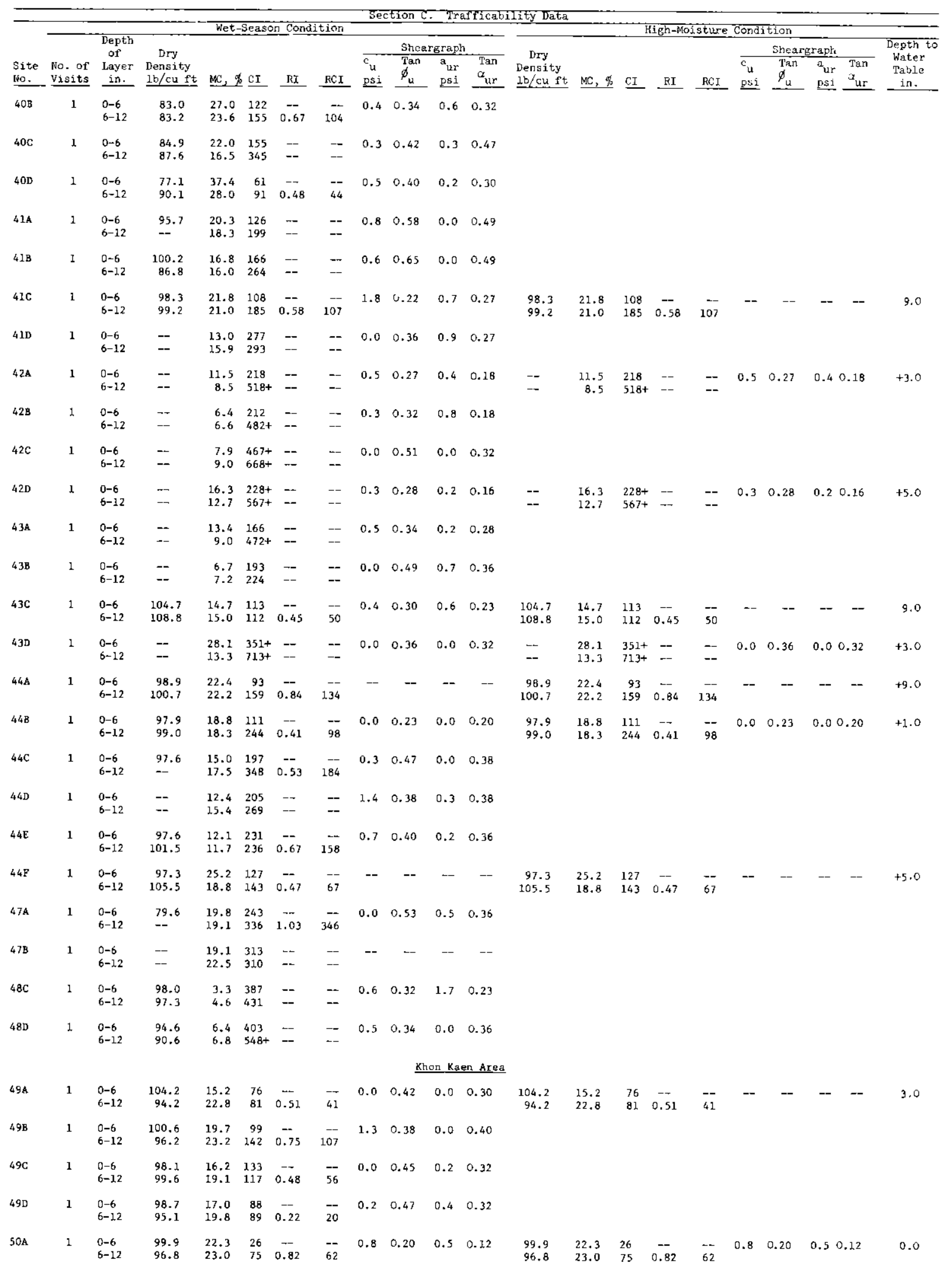




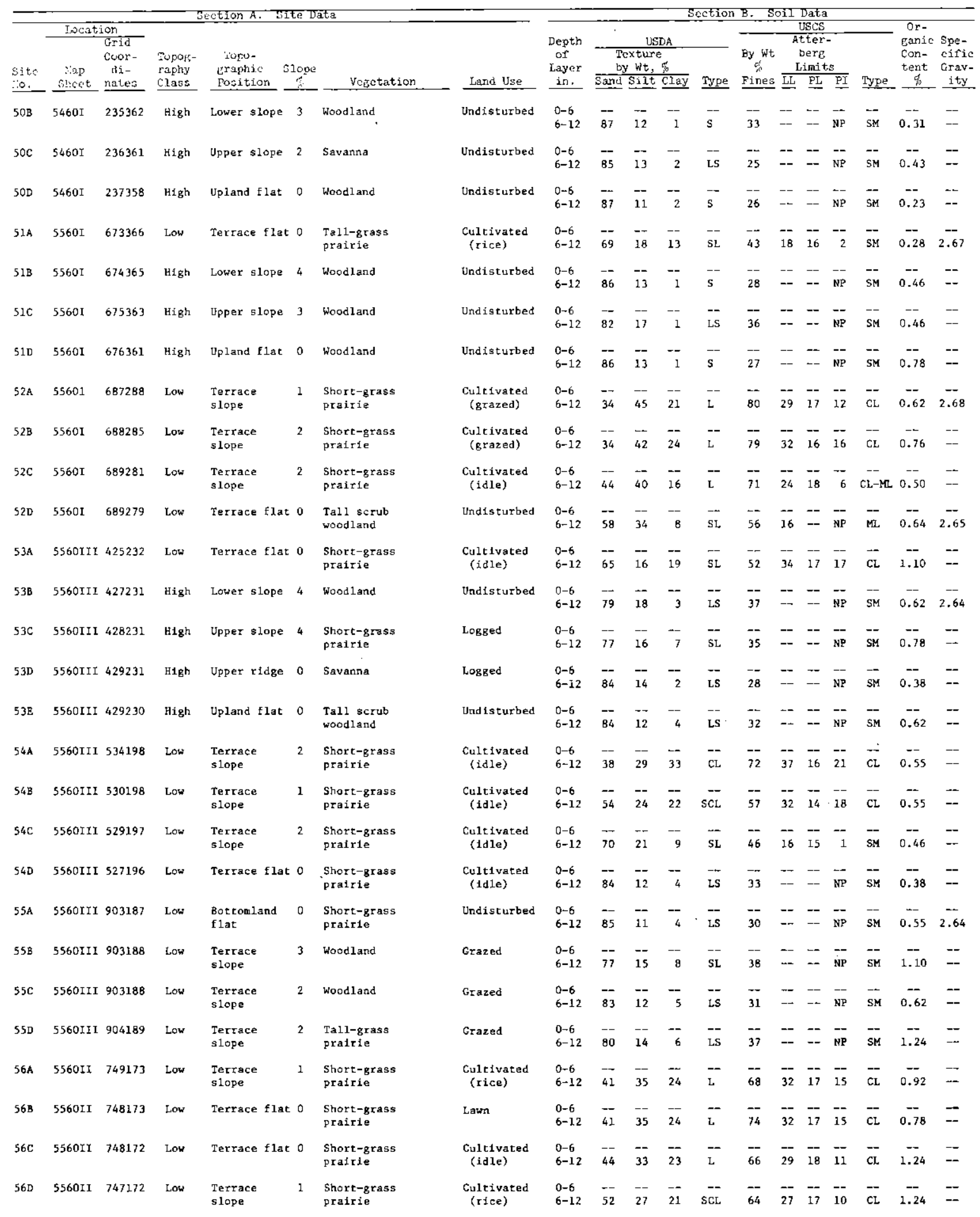




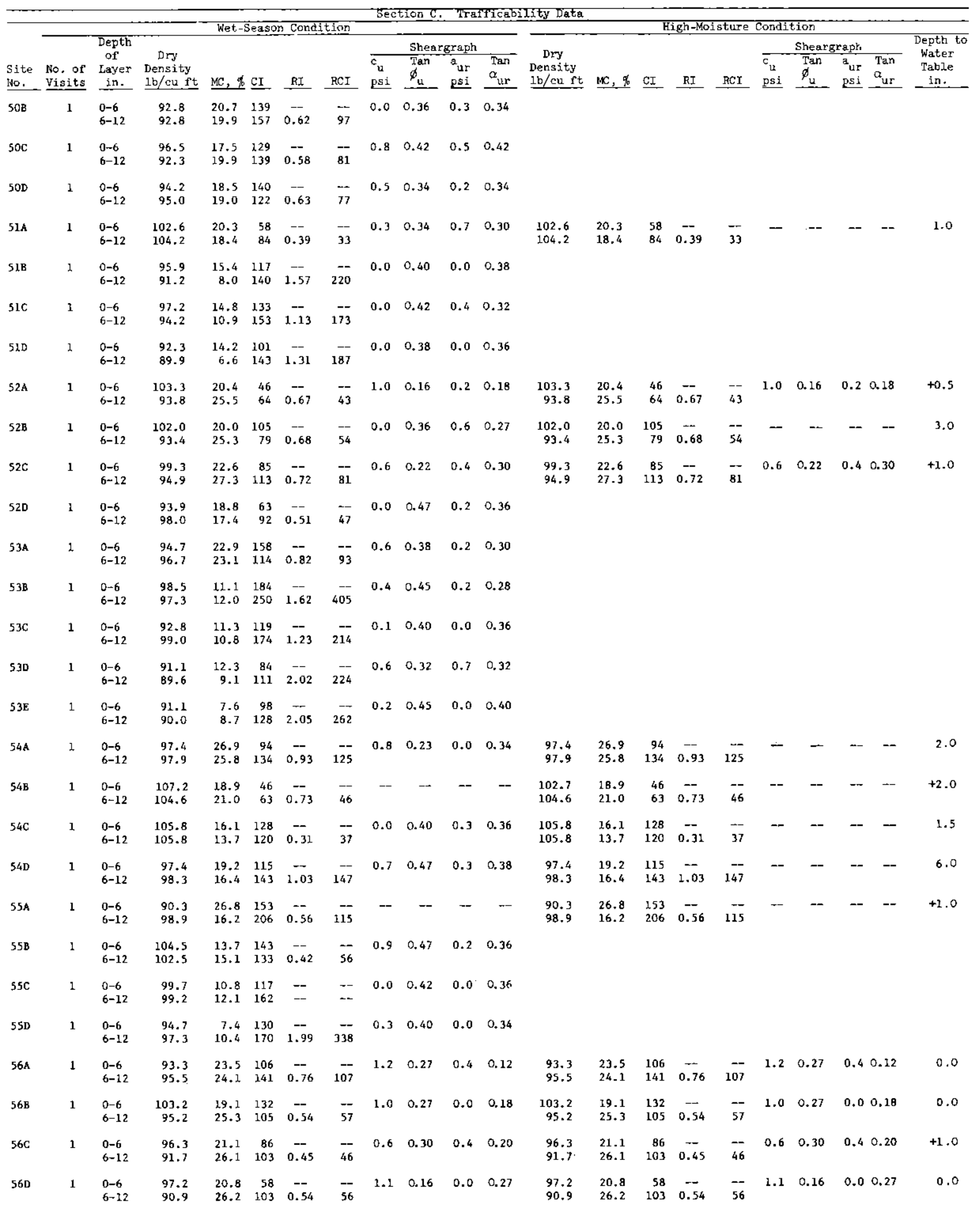


Table A3

Surface Composition Study

Sunnary of Site, Soil, and Trafficability Data

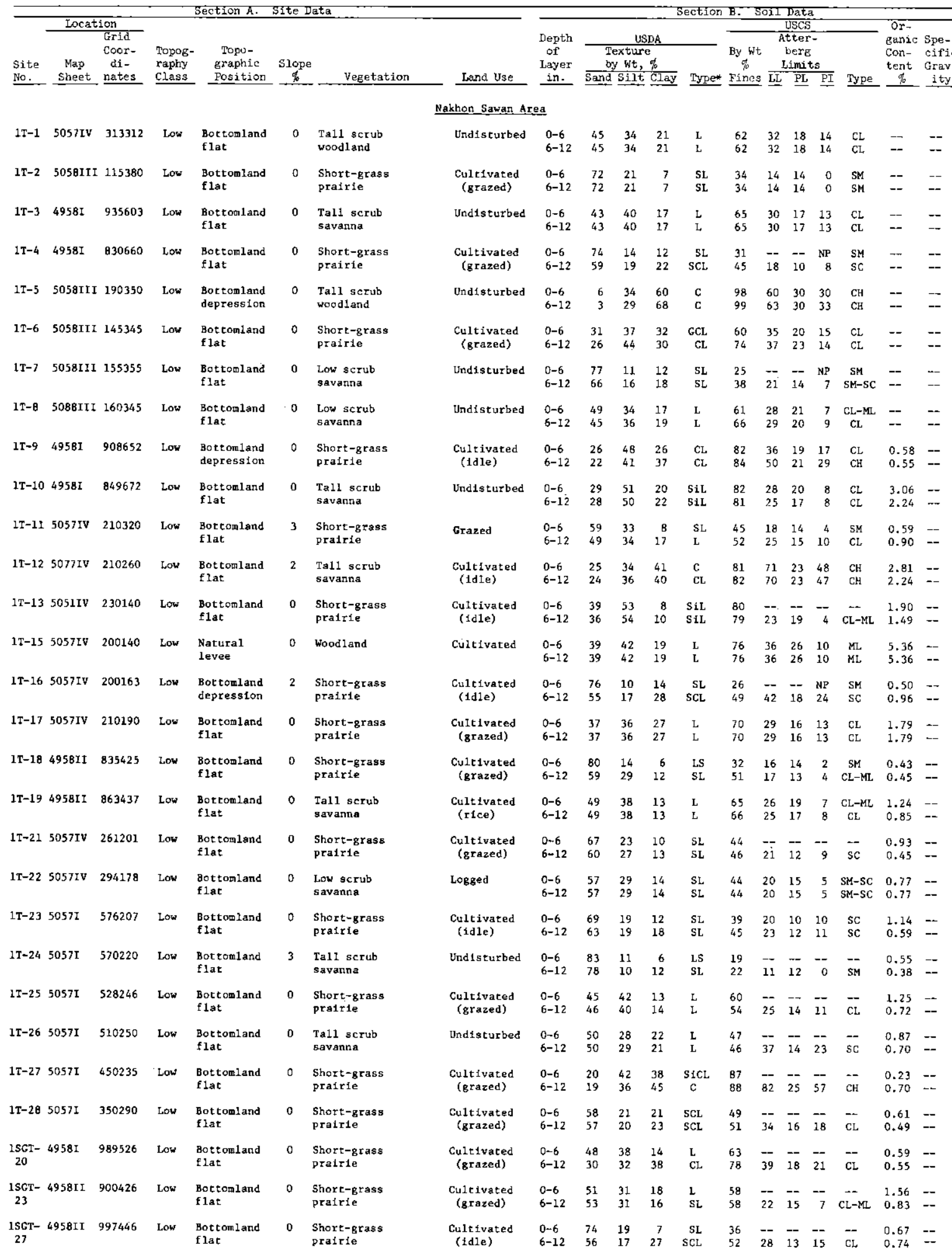




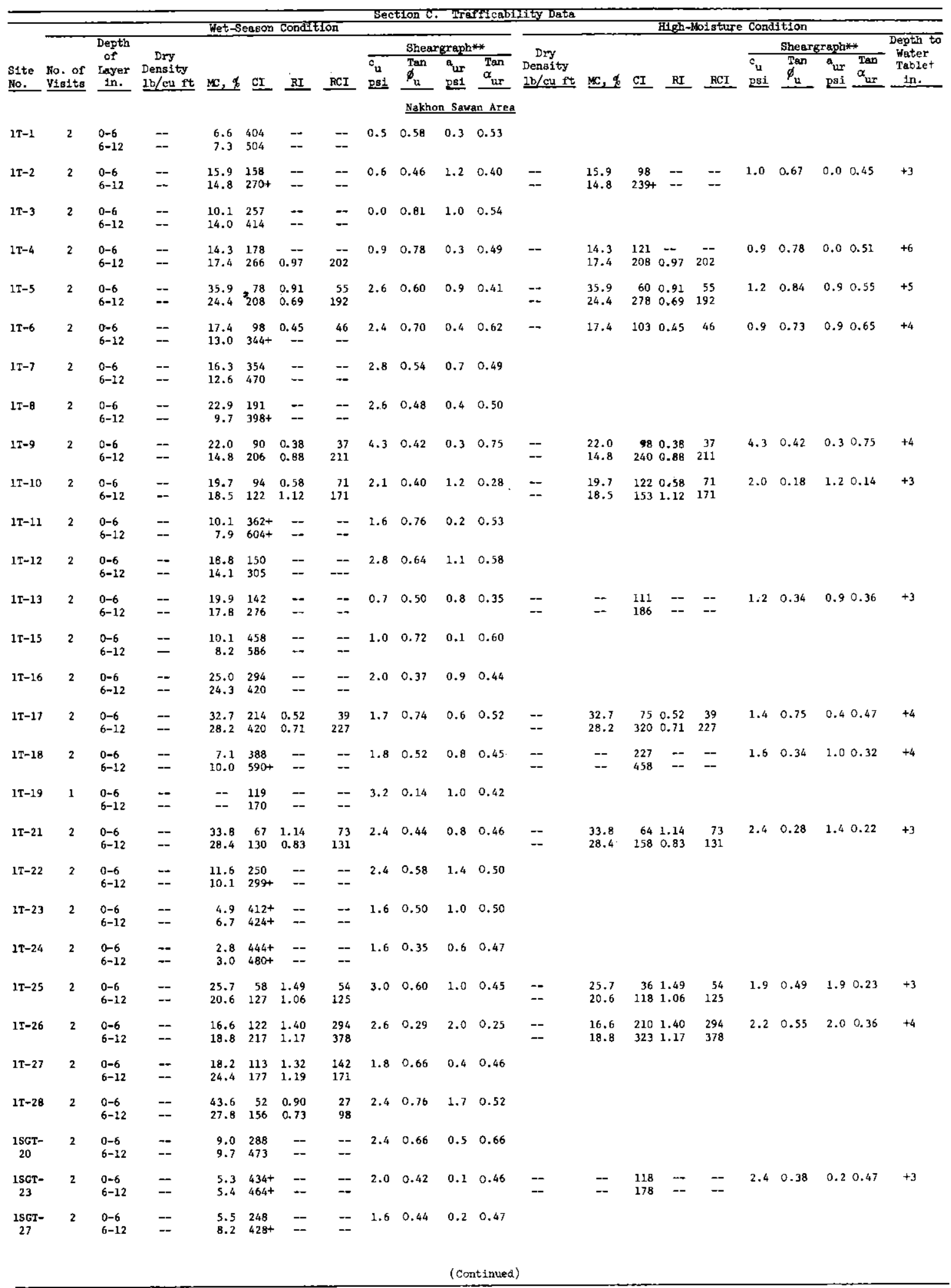

** $c_{u}$, ultimate soil-to-soll cohesion; $\phi_{u}$, uItimate soil-to-soil angle of internal friction; a , ultimate soil-to-rubber adhesion; $\alpha_{u r}$, ultimate soil-to-rubber engle of friction.

$t$ Plus (t) denotes depth of water above surface. 


\begin{tabular}{|c|c|c|c|c|c|c|c|c|c|c|c|c|c|c|c|c|c|c|c|}
\hline \multirow[b]{3}{*}{$\begin{array}{l}\text { Sitc } \\
\text { iio. } \\
\end{array}$} & \multirow{2}{*}{\multicolumn{2}{|c|}{ Location }} & & Section $A$. & te & & & \multirow[b]{3}{*}{$\begin{array}{l}\text { Depth } \\
\text { of } \\
\text { Layer } \\
\text { in. }\end{array}$} & \multirow{2}{*}{\multicolumn{9}{|c|}{ Section B. Soil Data }} & \multirow{3}{*}{$\begin{array}{l}\text { Or- } \\
\text { ganic } \\
\text { Con- } \\
\text { tent }\end{array}$} & \\
\hline & & & \multirow[b]{2}{*}{$\begin{array}{l}\text { Topog- } \\
\text { raphy } \\
\text { Class } \\
\end{array}$} & \multirow[b]{2}{*}{$\begin{array}{l}\text { Topo- } \\
\text { graphic } \\
\text { Position }\end{array}$} & \multirow[b]{2}{*}{$\begin{array}{c}\text { Slope } \\
\%\end{array}$} & \multirow[b]{2}{*}{ Vegetation } & \multirow[b]{2}{*}{ Land Use } & & & & & & & & & & & & \multirow[b]{2}{*}{$\begin{array}{l}\text { Spe- } \\
\text { cific } \\
\text { Grav- } \\
\text { ity }\end{array}$} \\
\hline & $\begin{array}{l}\text { Kap } \\
\text { Skcet }\end{array}$ & $\begin{array}{c}\text { Coor- } \\
\text { di- } \\
\text { nates }\end{array}$ & & & & & & & \multicolumn{3}{|c|}{$\begin{array}{c}\text { USSDA } \\
\text { Texture } \\
\text { by Wit, } \% \\
\end{array}$} & Type & $\begin{array}{l}\text { By wt } \\
\% \\
\text { Fines }\end{array}$ & & $\frac{\text { Limits }}{\mathrm{LL}}$ & $\frac{\mathrm{ts}}{\mathrm{PI}}$ & Type & & \\
\hline $\begin{array}{l}\text { 1SGT- } \\
30\end{array}$ & $5057 \mathrm{IV}$ & 233146 & Low & $\begin{array}{l}\text { Bottomland } \\
\text { flat }\end{array}$ & 0 & $\begin{array}{l}\text { Short-grass } \\
\text { prairie }\end{array}$ & $\begin{array}{l}\text { Cultivated } \\
\text { (grazed) }\end{array}$ & $\begin{array}{l}0-6 \\
6-12\end{array}$ & $\begin{array}{l}58 \\
40\end{array}$ & $\begin{array}{l}36 \\
46\end{array}$ & $\begin{array}{r}6 \\
14\end{array}$ & $\begin{array}{l}\text { SL } \\
\text { L }\end{array}$ & $\begin{array}{l}49 \\
67\end{array}$ & $\overline{16}$ & $\overline{12}$ & $\overline{4}$ & $\overline{C L-M I}$ & $\begin{array}{l}0.45 \\
0.28\end{array}$ & $\begin{array}{l}-- \\
--\end{array}$ \\
\hline & & & & & & & $\underline{\text { Lap Bur1 Area }}$ & & & & & & & & & & & & \\
\hline $2 \mathrm{~T}-1$ & $5153 \mathrm{IV}$ & 825794 & Low & $\begin{array}{l}\text { Bottomland } \\
\text { flat }\end{array}$ & 0 & $\begin{array}{l}\text { Short-grass } \\
\text { prairfe }\end{array}$ & $\begin{array}{l}\text { Cultivated } \\
\text { (idle) }\end{array}$ & $\begin{array}{l}0-6 \\
6-12\end{array}$ & $\begin{array}{l}6 \\
6\end{array}$ & $\begin{array}{l}62 \\
62\end{array}$ & $\begin{array}{l}32 \\
32\end{array}$ & $\begin{array}{l}\text { SiCL } \\
\text { SiCL }\end{array}$ & $\begin{array}{l}96 \\
96\end{array}$ & $\begin{array}{l}61 \\
61\end{array}$ & $\begin{array}{l}23 \\
23\end{array}$ & $\begin{array}{l}38 \\
38\end{array}$ & $\begin{array}{l}\mathrm{CH} \\
\mathrm{CH}\end{array}$ & -- & $\begin{array}{l}2.65 \\
2.65\end{array}$ \\
\hline $2 \Upsilon-2$ & $5153 \mathrm{IV}$ & 715743 & Low & $\begin{array}{l}\text { Bottomland } \\
\text { flat }\end{array}$ & 0 & $\begin{array}{l}\text { Short-grass } \\
\text { prairie }\end{array}$ & $\begin{array}{l}\text { Cultivated } \\
\text { (grazed) }\end{array}$ & $\begin{array}{l}0-6 \\
6-12\end{array}$ & $\begin{array}{l}14 \\
14\end{array}$ & $\begin{array}{l}59 \\
59\end{array}$ & $\begin{array}{l}27 \\
27\end{array}$ & $\begin{array}{l}\text { SiCL } \\
\text { SICL }\end{array}$ & $\begin{array}{l}90 \\
90\end{array}$ & $\begin{array}{l}57 \\
57\end{array}$ & $\begin{array}{l}29 \\
29\end{array}$ & $\begin{array}{l}28 \\
28\end{array}$ & $\begin{array}{l}\mathrm{CH} \\
\mathrm{CH}\end{array}$ & -- & $\begin{array}{l}2.52 \\
2.52\end{array}$ \\
\hline $2 \mathrm{~T}-3$ & $51531 \mathrm{~V}$ & 750830 & Low & $\begin{array}{l}\text { Bottomland } \\
\text { flat }\end{array}$ & 0 & $\begin{array}{l}\text { Short-grass } \\
\text { prafirie }\end{array}$ & $\begin{array}{l}\text { Cultivated } \\
\text { (idie) }\end{array}$ & $\begin{array}{l}0-6 \\
6-12\end{array}$ & $\begin{array}{l}5 \\
5\end{array}$ & $\begin{array}{l}65 \\
65\end{array}$ & $\begin{array}{l}30 \\
30\end{array}$ & $\begin{array}{l}\text { SiCL } \\
\text { SiCL }\end{array}$ & $\begin{array}{l}97 \\
97\end{array}$ & $\begin{array}{l}64 \\
64\end{array}$ & $\begin{array}{l}30 \\
30\end{array}$ & $\begin{array}{l}34 \\
34\end{array}$ & $\begin{array}{l}\mathrm{CH} \\
\mathrm{CH}\end{array}$ & -- & $\begin{array}{l}2.50 \\
2.50\end{array}$ \\
\hline $2 T-4$ & $51531 V$ & 786804 & Low & $\begin{array}{l}\text { Bottomland } \\
\text { flat }\end{array}$ & 0 & $\begin{array}{l}\text { Short-grass } \\
\text { prairle }\end{array}$ & $\begin{array}{l}\text { Cultivated } \\
\text { (Idle) }\end{array}$ & $\begin{array}{l}0-6 \\
6-12\end{array}$ & $\begin{array}{l}15 \\
18\end{array}$ & $\begin{array}{l}50 \\
48\end{array}$ & $\begin{array}{l}35 \\
34\end{array}$ & $\begin{array}{l}\text { S1CL } \\
\text { SiCL }\end{array}$ & $\begin{array}{l}89 \\
86\end{array}$ & $\begin{array}{l}62 \\
60\end{array}$ & $\begin{array}{l}27 \\
26\end{array}$ & $\begin{array}{l}35 \\
34\end{array}$ & $\begin{array}{l}\mathrm{CH} \\
\mathrm{CH}\end{array}$ & -- & $\begin{array}{l}2.69 \\
2.76\end{array}$ \\
\hline $2 T-5$ & $5154 \mathrm{I}$ & 085175 & Low & $\begin{array}{l}\text { Bottomland } \\
\text { flat }\end{array}$ & 0 & $\begin{array}{l}\text { Short-grass } \\
\text { prairie }\end{array}$ & $\begin{array}{l}\text { Cultivated } \\
\text { (Idle) }\end{array}$ & $\begin{array}{l}0-6 \\
6-12\end{array}$ & $\begin{array}{l}24 \\
24\end{array}$ & $\begin{array}{l}69 \\
69\end{array}$ & 7 & $\begin{array}{l}\text { SiL } \\
\text { S1L }\end{array}$ & $\begin{array}{l}83 \\
83\end{array}$ & $\begin{array}{l}31 \\
31\end{array}$ & $\begin{array}{l}18 \\
18\end{array}$ & $\begin{array}{l}13 \\
13\end{array}$ & $\begin{array}{l}\mathrm{CL} \\
\mathrm{CL}\end{array}$ & -- & $\begin{array}{l}2.64 \\
2.64\end{array}$ \\
\hline $2 T-6$ & $5154 \mathrm{I}$ & 100135 & Low & $\begin{array}{l}\text { Bot tomland } \\
\text { flat }\end{array}$ & 0 & $\begin{array}{l}\text { Short-grass } \\
\text { prairfe }\end{array}$ & $\begin{array}{l}\text { Cultivated } \\
\text { (idle) }\end{array}$ & $\begin{array}{l}0-6 \\
6-12\end{array}$ & $\begin{array}{l}26 \\
25\end{array}$ & $\begin{array}{l}62 \\
60\end{array}$ & $\begin{array}{l}12 \\
15\end{array}$ & $\begin{array}{l}\text { Sí } \\
\text { SiL }\end{array}$ & $\begin{array}{l}78 \\
74\end{array}$ & $\begin{array}{l}58 \\
62\end{array}$ & $\begin{array}{l}23 \\
23\end{array}$ & $\begin{array}{l}35 \\
39\end{array}$ & $\begin{array}{l}\mathrm{CH} \\
\mathrm{CH}\end{array}$ & $\begin{array}{l}-- \\
--\end{array}$ & $\begin{array}{l}2.75 \\
2.74\end{array}$ \\
\hline $2 T-7$ & 51541 & 080117 & Low & $\begin{array}{l}\text { Bottomland } \\
\text { flat }\end{array}$ & 0 & $\begin{array}{l}\text { Short-grass } \\
\text { prairie }\end{array}$ & $\begin{array}{l}\text { Cultivated } \\
\text { (IdIe) }\end{array}$ & $\begin{array}{l}0-6 \\
6-12\end{array}$ & $\begin{array}{l}24 \\
24\end{array}$ & $\begin{array}{l}66 \\
66\end{array}$ & $\begin{array}{l}10 \\
10\end{array}$ & $\begin{array}{l}\text { S1L } \\
\text { SIL }\end{array}$ & $\begin{array}{l}83 \\
83\end{array}$ & $\begin{array}{l}40 \\
40\end{array}$ & $\begin{array}{l}18 \\
18\end{array}$ & $\begin{array}{l}22 \\
22\end{array}$ & $\begin{array}{l}\mathrm{CL} \\
\mathrm{CL}\end{array}$ & -- & $\begin{array}{l}2.69 \\
2.69\end{array}$ \\
\hline $2 \mathrm{~T}-8$ & $5154 \mathrm{I}$ & 142114 & Low & $\begin{array}{l}\text { Bottomland } \\
\text { flat }\end{array}$ & 0 & $\begin{array}{l}\text { Short-grass } \\
\text { prairie }\end{array}$ & $\begin{array}{l}\text { CuItívated } \\
\text { (grazed) }\end{array}$ & $\begin{array}{l}0-6 \\
6-12\end{array}$ & $\begin{array}{l}7 \\
8\end{array}$ & $\begin{array}{l}85 \\
53\end{array}$ & $\begin{array}{l}28 \\
39\end{array}$ & $\begin{array}{l}\text { SiCL } \\
\text { SiCL }\end{array}$ & $\begin{array}{l}96 \\
94\end{array}$ & $\begin{array}{l}57 \\
61\end{array}$ & $\begin{array}{l}29 \\
29\end{array}$ & $\begin{array}{l}28 \\
32\end{array}$ & $\begin{array}{l}\mathrm{CH} \\
\mathrm{CH}\end{array}$ & -- & $\begin{array}{l}2.69 \\
2.71\end{array}$ \\
\hline $2 \mathrm{~T}-9$ & $5154 \mathrm{I}$ & 040210 & Low & $\begin{array}{l}\text { Bottomland } \\
\text { flat }\end{array}$ & 0 & $\begin{array}{l}\text { Short-grass } \\
\text { prairie }\end{array}$ & $\begin{array}{l}\text { Cultivated } \\
\text { (grazed) }\end{array}$ & $\begin{array}{l}0-6 \\
6-12\end{array}$ & $\begin{array}{l}25 \\
25\end{array}$ & $\begin{array}{l}65 \\
65\end{array}$ & $\begin{array}{l}10 \\
10\end{array}$ & $\begin{array}{l}\text { S1L } \\
\text { S1L }\end{array}$ & $\begin{array}{l}82 \\
82\end{array}$ & $\begin{array}{l}36 \\
36\end{array}$ & $\begin{array}{l}21 \\
21\end{array}$ & $\begin{array}{l}15 \\
15\end{array}$ & $\begin{array}{l}\mathrm{CL} \\
\mathrm{CL}\end{array}$ & -- & $\begin{array}{l}2.68 \\
2.68\end{array}$ \\
\hline $2 T-10$ & $5154 \mathrm{I}$ & 070080 & Low & $\begin{array}{l}\text { Bottomland } \\
\text { flat }\end{array}$ & 0 & $\begin{array}{l}\text { Short-grass } \\
\text { prairie }\end{array}$ & $\begin{array}{l}\text { Culclvated } \\
\text { (1dle) }\end{array}$ & $\begin{array}{l}0-6 \\
6-12\end{array}$ & $\begin{array}{l}40 \\
40\end{array}$ & $\begin{array}{l}55 \\
55\end{array}$ & $\begin{array}{l}5 \\
5\end{array}$ & $\begin{array}{l}\text { SfL } \\
\text { SIL }\end{array}$ & $\begin{array}{l}56 \\
56\end{array}$ & $\begin{array}{l}17 \\
17\end{array}$ & $\begin{array}{l}15 \\
15\end{array}$ & $\begin{array}{l}2 \\
2\end{array}$ & $\begin{array}{l}M L \\
M L\end{array}$ & $=$ & $\begin{array}{l}2.75 \\
2.75\end{array}$ \\
\hline $2 T-11$ & 5154 II & 080990 & Low & $\begin{array}{l}\text { Bottomland } \\
\text { flat }\end{array}$ & 0 & $\begin{array}{l}\text { Short-grass } \\
\text { prairie }\end{array}$ & $\begin{array}{l}\text { Cultivated } \\
\text { (idle) }\end{array}$ & $\begin{array}{l}0-6 \\
6-12\end{array}$ & $\begin{array}{l}55 \\
59\end{array}$ & $\begin{array}{l}39 \\
35\end{array}$ & $\begin{array}{l}6 \\
6\end{array}$ & $\begin{array}{l}\text { SL } \\
\text { SL }\end{array}$ & $\begin{array}{l}53 \\
49\end{array}$ & -- & -- & - & SM & -- & $\begin{array}{l}2.63 \\
2.60\end{array}$ \\
\hline $2 T-12$ & $5154 \mathrm{II}$ & 000000 & Low & $\begin{array}{l}\text { Bottomland } \\
\text { flat }\end{array}$ & 0 & $\begin{array}{l}\text { Short-grass } \\
\text { pra1rie }\end{array}$ & $\begin{array}{l}\text { Cultivated } \\
\text { (grazed) }\end{array}$ & $\begin{array}{l}0-6 \\
6-12\end{array}$ & $\begin{array}{l}35 \\
35\end{array}$ & $\begin{array}{l}54 \\
54\end{array}$ & $\begin{array}{l}11 \\
11\end{array}$ & $\begin{array}{l}\text { SiL } \\
\text { SiL }\end{array}$ & $\begin{array}{l}73 \\
73\end{array}$ & $\begin{array}{l}25 \\
25\end{array}$ & $\begin{array}{l}19 \\
19\end{array}$ & $\begin{array}{l}6 \\
6\end{array}$ & $\begin{array}{l}\text { CL-ML } \\
\text { CL-ML }\end{array}$ & -- & $\begin{array}{l}2.64 \\
2.64\end{array}$ \\
\hline $2 \mathrm{~T}-13$ & $5154 \mathrm{II}$ & 970933 & Low & $\begin{array}{l}\text { Bottomland } \\
\text { flat }\end{array}$ & 0 & $\begin{array}{l}\text { Short-grass } \\
\text { prairle }\end{array}$ & $\begin{array}{l}\text { Cultivated } \\
\text { (idle) }\end{array}$ & $\begin{array}{l}0-6 \\
6-12\end{array}$ & $\begin{array}{l}21 \\
21\end{array}$ & $\begin{array}{l}48 \\
46\end{array}$ & $\begin{array}{l}31 \\
33\end{array}$ & $\begin{array}{l}\mathrm{CL} \\
\mathrm{CL}\end{array}$ & $\begin{array}{l}84 \\
83\end{array}$ & $\begin{array}{l}42 \\
43\end{array}$ & $\begin{array}{l}26 \\
25\end{array}$ & $\begin{array}{l}16 \\
18\end{array}$ & $\begin{array}{l}\mathrm{CL} \\
\mathrm{CL}\end{array}$ & $=$ & $\begin{array}{l}2.63 \\
2.63\end{array}$ \\
\hline $2 T-14$ & 515411 & 000930 & Low & $\begin{array}{l}\text { Bot tomland } \\
\text { flat }\end{array}$ & 0 & $\begin{array}{l}\text { Short-grass } \\
\text { prairie }\end{array}$ & $\begin{array}{l}\text { Cultivated } \\
\text { (Idle) }\end{array}$ & $\begin{array}{l}0-6 \\
6-12\end{array}$ & $\begin{array}{l}20 \\
15\end{array}$ & $\begin{array}{l}61 \\
54\end{array}$ & $\begin{array}{l}19 \\
31\end{array}$ & $\begin{array}{r}\text { SiL } \\
\text { SiCL }\end{array}$ & $\begin{array}{l}85 \\
89\end{array}$ & $\begin{array}{l}28 \\
41\end{array}$ & $\begin{array}{l}19 \\
23\end{array}$ & $\begin{array}{r}9 \\
18\end{array}$ & $\begin{array}{l}\mathrm{CL} \\
\mathrm{CL}\end{array}$ & -- & $\begin{array}{l}2.64 \\
2.68\end{array}$ \\
\hline $2 T-15$ & $5154 \mathrm{II}$ & 020875 & Low & $\begin{array}{l}\text { Bottomland } \\
\text { flat }\end{array}$ & 0 & $\begin{array}{l}\text { Short-grass } \\
\text { prairie }\end{array}$ & $\begin{array}{l}\text { Cultivated } \\
\text { (grazed) }\end{array}$ & $\begin{array}{l}0-6 \\
6-12\end{array}$ & $\begin{array}{r}6 \\
12\end{array}$ & $\begin{array}{l}60 \\
58\end{array}$ & $\begin{array}{l}34 \\
30\end{array}$ & $\begin{array}{l}\text { S1CL } \\
\text { SiCL }\end{array}$ & $\begin{array}{l}96 \\
92\end{array}$ & $\begin{array}{l}46 \\
45\end{array}$ & $\begin{array}{l}26 \\
25\end{array}$ & $\begin{array}{l}20 \\
20\end{array}$ & $\begin{array}{l}\mathrm{CL} \\
\mathrm{CL}\end{array}$ & $\begin{array}{l}-- \\
--\end{array}$ & $\begin{array}{l}2.68 \\
2.73\end{array}$ \\
\hline $2 T-16$ & $5154 \mathrm{II}$ & 050860 & Low & $\begin{array}{l}\text { Bottomland } \\
\text { flat }\end{array}$ & 0 & $\begin{array}{l}\text { Short-grass } \\
\text { prairie }\end{array}$ & $\begin{array}{l}\text { Cultivated } \\
\text { (idle) }\end{array}$ & $\begin{array}{l}0-6 \\
6-12\end{array}$ & $\begin{array}{l}8 \\
8\end{array}$ & $\begin{array}{l}67 \\
67\end{array}$ & $\begin{array}{l}25 \\
25\end{array}$ & $\begin{array}{l}\text { SiL } \\
\text { S1L }\end{array}$ & $\begin{array}{l}94 \\
94\end{array}$ & $\begin{array}{l}53 \\
53\end{array}$ & $\begin{array}{l}25 \\
25\end{array}$ & $\begin{array}{l}28 \\
28\end{array}$ & $\begin{array}{l}\mathrm{CH} \\
\mathrm{CH}\end{array}$ & -- & $\begin{array}{l}2.65 \\
2.65\end{array}$ \\
\hline $2 T-17$ & \$154III & 850985 & Low & $\begin{array}{l}\text { Botromland } \\
\text { flat }\end{array}$ & 0 & $\begin{array}{l}\text { Short-grass } \\
\text { prairie }\end{array}$ & $\begin{array}{l}\text { Cultivated } \\
\text { (grazed) }\end{array}$ & $\begin{array}{l}0-6 \\
6-12\end{array}$ & $\begin{array}{l}12 \\
12\end{array}$ & $\begin{array}{l}69 \\
69\end{array}$ & $\begin{array}{l}19 \\
19\end{array}$ & $\begin{array}{l}\text { SiL } \\
\text { SiL }\end{array}$ & $\begin{array}{l}96 \\
96\end{array}$ & $\begin{array}{l}45 \\
45\end{array}$ & $\begin{array}{l}22 \\
22\end{array}$ & $\begin{array}{l}23 \\
23\end{array}$ & $\begin{array}{l}\mathrm{CL} \\
\mathrm{CL}\end{array}$ & -- & $\begin{array}{l}2.67 \\
2.67\end{array}$ \\
\hline $2 \mathrm{~T}-18$ & 5154 III & 211945 & Low & $\begin{array}{l}\text { Bottomland } \\
\text { flat }\end{array}$ & 0 & $\begin{array}{l}\text { Short-grass } \\
\text { prairie }\end{array}$ & $\begin{array}{l}\text { Cultivated } \\
\text { (Idle) }\end{array}$ & $\begin{array}{l}0-6 \\
6-12\end{array}$ & $\begin{array}{l}7 \\
7\end{array}$ & $\begin{array}{l}69 \\
69\end{array}$ & $\begin{array}{l}24 \\
24\end{array}$ & $\begin{array}{l}\text { SiL } \\
\text { SiL }\end{array}$ & $\begin{array}{l}96 \\
96\end{array}$ & $\begin{array}{l}49 \\
49\end{array}$ & $\begin{array}{l}25 \\
25\end{array}$ & $\begin{array}{l}24 \\
24\end{array}$ & $\begin{array}{l}\text { CL } \\
\mathrm{CL}\end{array}$ & -- & $\begin{array}{l}2.68 \\
2.68\end{array}$ \\
\hline $2 T-19$ & $5154 \mathrm{III}$ & 793905 & Low & $\begin{array}{l}\text { Bottomland } \\
\text { flat }\end{array}$ & 0 & $\begin{array}{l}\text { Short-grass } \\
\text { prairle }\end{array}$ & $\begin{array}{l}\text { Cultivated } \\
\text { (1dle) }\end{array}$ & $\begin{array}{l}0-6 \\
6-12\end{array}$ & $\begin{array}{l}16 \\
16\end{array}$ & $\begin{array}{l}64 \\
64\end{array}$ & $\begin{array}{l}20 \\
20\end{array}$ & $\begin{array}{l}\text { SiL } \\
\text { SiL }\end{array}$ & $\begin{array}{l}89 \\
B 9\end{array}$ & $\begin{array}{l}44 \\
44\end{array}$ & $\begin{array}{l}20 \\
20\end{array}$ & $\begin{array}{l}24 \\
24\end{array}$ & $\begin{array}{l}\mathrm{CL} \\
\mathrm{CL}\end{array}$ & $\overline{--}$ & $\begin{array}{l}2.67 \\
2.67\end{array}$ \\
\hline $2 T-20$ & $5154 \mathrm{IV}$ & 870130 & Low & $\begin{array}{l}\text { Bot tom } 1 \text { and } \\
\text { flat }\end{array}$ & 0 & $\begin{array}{l}\text { Short-grass } \\
\text { prairie }\end{array}$ & $\begin{array}{l}\text { Cultivated } \\
\text { (grazed) }\end{array}$ & $\begin{array}{l}0-6 \\
6-12\end{array}$ & $\begin{array}{l}13 \\
13\end{array}$ & $\begin{array}{l}65 \\
65\end{array}$ & $\begin{array}{l}22 \\
22\end{array}$ & $\begin{array}{l}\text { SIL } \\
\text { SIL }\end{array}$ & $\begin{array}{l}93 \\
93\end{array}$ & $\begin{array}{l}62 \\
62\end{array}$ & $\begin{array}{l}25 \\
25\end{array}$ & $\begin{array}{l}37 \\
37\end{array}$ & $\begin{array}{l}\mathrm{CH} \\
\mathrm{CH}\end{array}$ & -- & $\begin{array}{l}2.59 \\
2.59\end{array}$ \\
\hline $2 \mathrm{~T}-21$ & $5155 \mathrm{II}$ & 913260 & Low & $\begin{array}{l}\text { Bottomland } \\
\text { flat }\end{array}$ & 0 & $\begin{array}{l}\text { Short-grass } \\
\text { prairie }\end{array}$ & $\begin{array}{l}\text { Cultivated } \\
\text { (Idle) }\end{array}$ & $\begin{array}{l}0-6 \\
6-12\end{array}$ & $\begin{array}{l}39 \\
39\end{array}$ & $\begin{array}{l}51 \\
51\end{array}$ & $\begin{array}{l}10 \\
10\end{array}$ & $\begin{array}{l}\text { SiL } \\
\text { SiL }\end{array}$ & $\begin{array}{l}70 \\
70\end{array}$ & $\begin{array}{l}59 \\
59\end{array}$ & $\begin{array}{l}32 \\
32\end{array}$ & $\begin{array}{l}27 \\
27\end{array}$ & $\begin{array}{l}\text { MH } \\
\text { MH }\end{array}$ & - & $\begin{array}{l}2.63 \\
2.63\end{array}$ \\
\hline $2 T-22$ & S155II & 935370 & Low & $\begin{array}{l}\text { Bot tomland } \\
\text { flat }\end{array}$ & 0 & $\begin{array}{l}\text { Short-grass } \\
\text { prairie }\end{array}$ & $\begin{array}{l}\text { Cultivated } \\
\text { (1dle) }\end{array}$ & $\begin{array}{l}0-6 \\
6-12\end{array}$ & $\begin{array}{l}34 \\
34\end{array}$ & $\begin{array}{l}59 \\
59\end{array}$ & $\frac{7}{7}$ & $\begin{array}{l}\text { SiL } \\
\text { SiL }\end{array}$ & $\begin{array}{l}74 \\
74\end{array}$ & $\begin{array}{l}29 \\
29\end{array}$ & $\begin{array}{l}18 \\
18\end{array}$ & $\begin{array}{l}11 \\
11\end{array}$ & $\begin{array}{l}\mathrm{CL} \\
\mathrm{CL}\end{array}$ & -- & $\begin{array}{l}2.63 \\
2.63\end{array}$ \\
\hline $2 T-23$ & 5IS5II & 010240 & Low & $\begin{array}{l}\text { Bott omland } \\
\text { depression }\end{array}$ & 0 & $\begin{array}{l}\text { Short-grass } \\
\text { prairle }\end{array}$ & $\begin{array}{l}\text { Cultivated } \\
\text { (idle) }\end{array}$ & $\begin{array}{l}0-6 \\
6-12\end{array}$ & $\begin{array}{l}46 \\
33\end{array}$ & $\begin{array}{l}42 \\
49\end{array}$ & $\begin{array}{l}12 \\
18\end{array}$ & $\begin{array}{l}\mathbf{L} \\
\mathbf{L}\end{array}$ & $\begin{array}{l}54 \\
73\end{array}$ & $\begin{array}{l}28 \\
37\end{array}$ & $\begin{array}{l}18 \\
19\end{array}$ & $\begin{array}{l}10 \\
19\end{array}$ & $\begin{array}{l}\mathrm{CL} \\
\mathrm{CL}\end{array}$ & -- & $\begin{array}{l}2.67 \\
2.65\end{array}$ \\
\hline $2 T-24$ & S155III & 680400 & Low & $\begin{array}{l}\text { Bottomland } \\
\text { flat }\end{array}$ & 0 & $\begin{array}{l}\text { Short-grass } \\
\text { prairie }\end{array}$ & $\begin{array}{l}\text { Cultivated } \\
\text { (grazed) }\end{array}$ & $\begin{array}{l}0-6 \\
6-12\end{array}$ & $\begin{array}{l}10 \\
10\end{array}$ & $\begin{array}{l}54 \\
54\end{array}$ & $\begin{array}{l}36 \\
36\end{array}$ & $\begin{array}{l}\text { SiCl } \\
\text { SICL }\end{array}$ & $\begin{array}{l}93 \\
93\end{array}$ & $\begin{array}{l}92 \\
92\end{array}$ & $\begin{array}{l}29 \\
29\end{array}$ & $\begin{array}{l}63 \\
63\end{array}$ & $\begin{array}{l}\mathrm{CH} \\
\mathrm{CH}\end{array}$ & -- & $\begin{array}{l}2.56 \\
2.56\end{array}$ \\
\hline $2 T-25$ & S155III & 680380 & Low & $\begin{array}{l}\text { Bottomland } \\
\text { flat }\end{array}$ & 0 & $\begin{array}{l}\text { Short-grass } \\
\text { prairie }\end{array}$ & $\begin{array}{l}\text { Cultivated } \\
\text { (grazed) }\end{array}$ & $\begin{array}{l}0-6 \\
6-12\end{array}$ & $\begin{array}{l}9 \\
9\end{array}$ & $\begin{array}{l}66 \\
66\end{array}$ & $\begin{array}{l}25 \\
25\end{array}$ & $\begin{array}{l}\text { SiCL } \\
\text { SiCL }\end{array}$ & $\begin{array}{l}95 \\
95\end{array}$ & $\begin{array}{l}47 \\
47\end{array}$ & $\begin{array}{l}26 \\
26\end{array}$ & $\begin{array}{l}21 \\
21\end{array}$ & $\begin{array}{l}\mathrm{CL} \\
\mathrm{CL}\end{array}$ & $\overline{-}$ & $\begin{array}{l}2.65 \\
2.65\end{array}$ \\
\hline $2 T-26$ & S155III & 820290 & Low & $\begin{array}{l}\text { Boztomland } \\
\text { Elat }\end{array}$ & 0 & $\begin{array}{l}\text { Short-grass } \\
\text { prairie }\end{array}$ & $\begin{array}{l}\text { Cultivated } \\
\text { (grazed) }\end{array}$ & $\begin{array}{l}0-6 \\
6-12\end{array}$ & $\begin{array}{l}22 \\
22\end{array}$ & $\begin{array}{l}59 \\
59\end{array}$ & $\begin{array}{l}19 \\
19\end{array}$ & $\begin{array}{l}\text { SiL } \\
\text { SiL }\end{array}$ & $\begin{array}{l}87 \\
87\end{array}$ & $\begin{array}{l}86 \\
86\end{array}$ & $\begin{array}{l}42 \\
42\end{array}$ & $\begin{array}{l}44 \\
44\end{array}$ & $\begin{array}{l}\mathrm{MH} \\
\mathrm{MH}\end{array}$ & -- & $\begin{array}{l}2.58 \\
2.58\end{array}$ \\
\hline $2 T-27$ & $5155 \mathrm{TV}$ & 780470 & Low & $\begin{array}{l}\text { Bot tomland } \\
\text { flat }\end{array}$ & 0 & $\begin{array}{l}\text { Short-grass } \\
\text { prairie }\end{array}$ & Undisturbed & $\begin{array}{l}0-6 \\
6-12\end{array}$ & $\begin{array}{l}25 \\
25\end{array}$ & $\begin{array}{l}64 \\
64\end{array}$ & $\begin{array}{l}11 \\
11\end{array}$ & $\begin{array}{l}\text { SiL } \\
\text { SiL }\end{array}$ & $\begin{array}{l}82 \\
82\end{array}$ & $\begin{array}{l}51 \\
51\end{array}$ & $\begin{array}{l}20 \\
20\end{array}$ & $\begin{array}{l}31 \\
31\end{array}$ & $\begin{array}{l}\mathrm{CH} \\
\mathrm{CH}\end{array}$ & - & $\begin{array}{l}2.61 \\
2.61\end{array}$ \\
\hline $2 T-28$ & $5155 \mathrm{IV}$ & 803437 & Low & $\begin{array}{l}\text { Bottomland } \\
\text { f1at }\end{array}$ & 5 & $\begin{array}{l}\text { Tall-grass } \\
\text { prairle }\end{array}$ & Undisturbed & $\begin{array}{l}0-6 \\
6-12\end{array}$ & $\begin{array}{l}36 \\
36\end{array}$ & $\begin{array}{l}56 \\
56\end{array}$ & $\begin{array}{l}8 \\
8\end{array}$ & $\begin{array}{l}\text { SiL } \\
\text { SiL }\end{array}$ & $\begin{array}{l}70 \\
70\end{array}$ & $\begin{array}{l}50 \\
50\end{array}$ & $\begin{array}{l}24 \\
24\end{array}$ & $\begin{array}{l}26 \\
26\end{array}$ & $\begin{array}{l}\mathrm{CH} \\
\mathrm{CH}\end{array}$ & -- & $\begin{array}{l}2.60 \\
2.60\end{array}$ \\
\hline $2 \mathrm{~T}-29$ & $51551 \mathrm{~V}$ & 780420 & Low & $\begin{array}{l}\text { Bottonland } \\
\text { flat }\end{array}$ & 0 & $\begin{array}{l}\text { Short-grass } \\
\text { prafrie }\end{array}$ & $\begin{array}{l}\text { Cultivated } \\
\text { (grazed) }\end{array}$ & $\begin{array}{l}0-6 \\
6-12\end{array}$ & $\begin{array}{l}26 \\
26\end{array}$ & $\begin{array}{l}65 \\
65\end{array}$ & $\stackrel{9}{9}$ & $\begin{array}{l}\text { Sil } \\
\text { SiL }\end{array}$ & $\begin{array}{l}80 \\
80\end{array}$ & $\begin{array}{l}35 \\
36\end{array}$ & $\begin{array}{l}17 \\
17\end{array}$ & $\begin{array}{l}19 \\
19\end{array}$ & $\begin{array}{l}\mathrm{CL} \\
\mathrm{CL}\end{array}$ & $\overline{-}$ & $\begin{array}{l}2.57 \\
2.57\end{array}$ \\
\hline
\end{tabular}




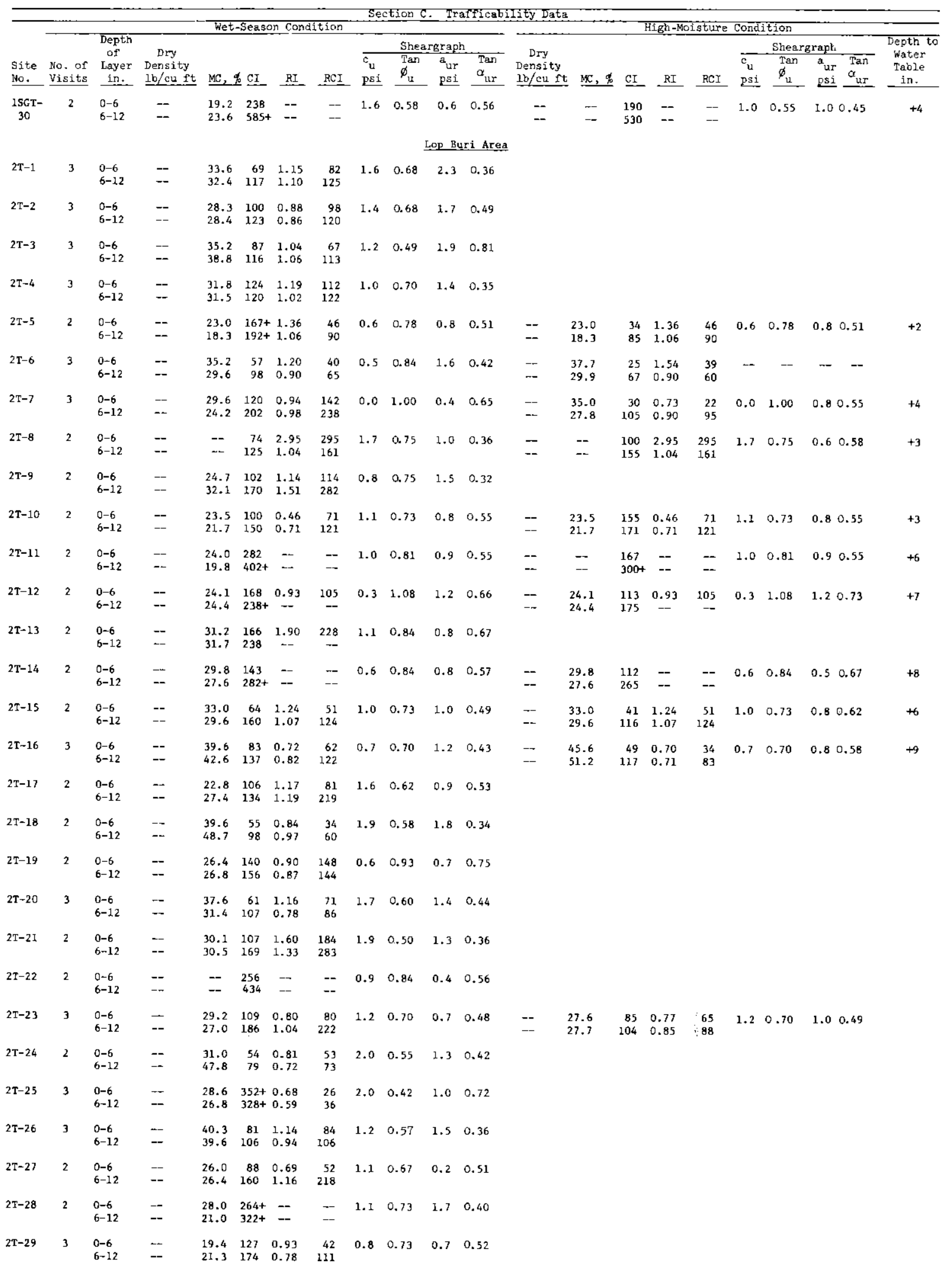


Table A3 (Continued)

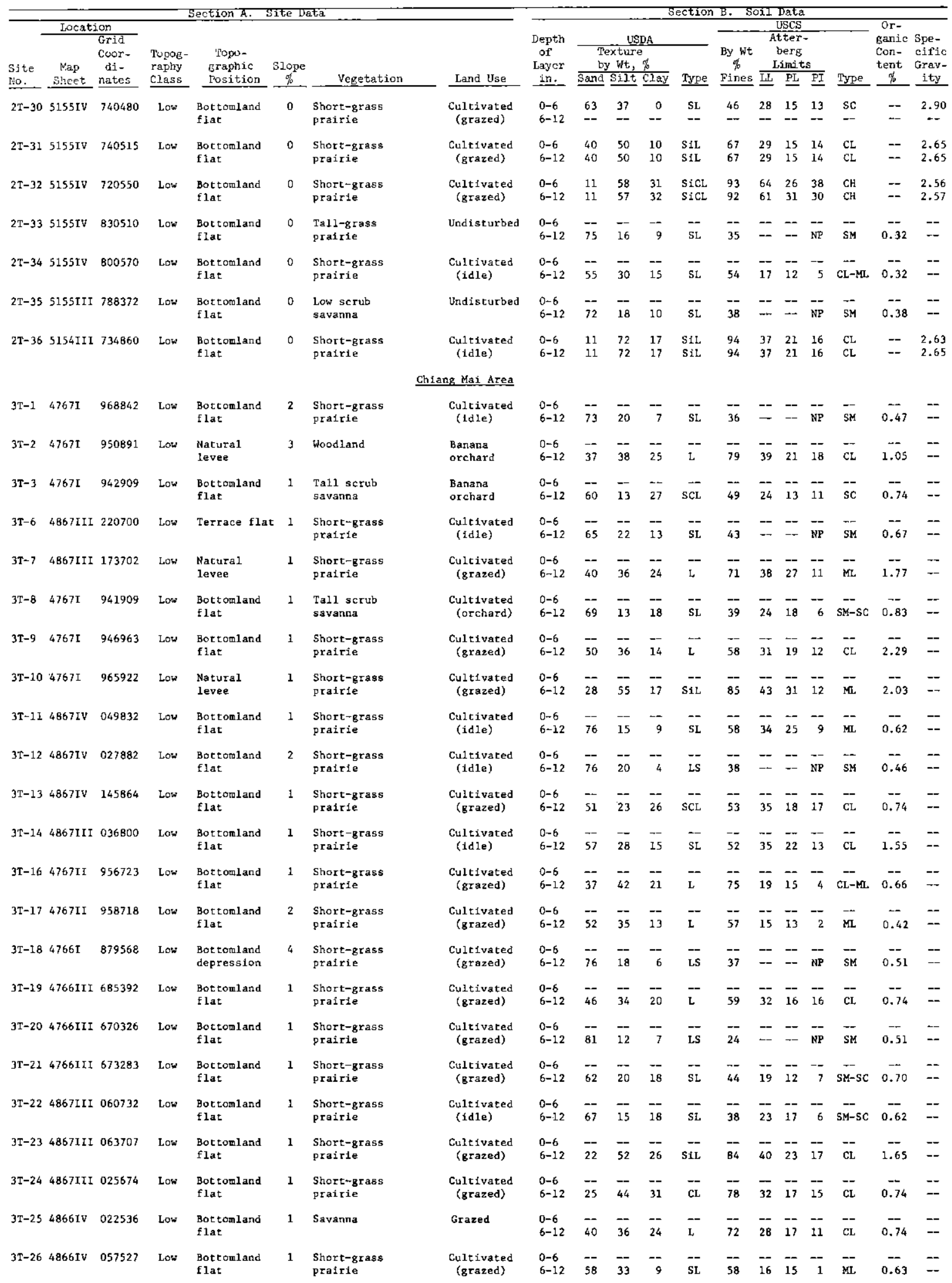




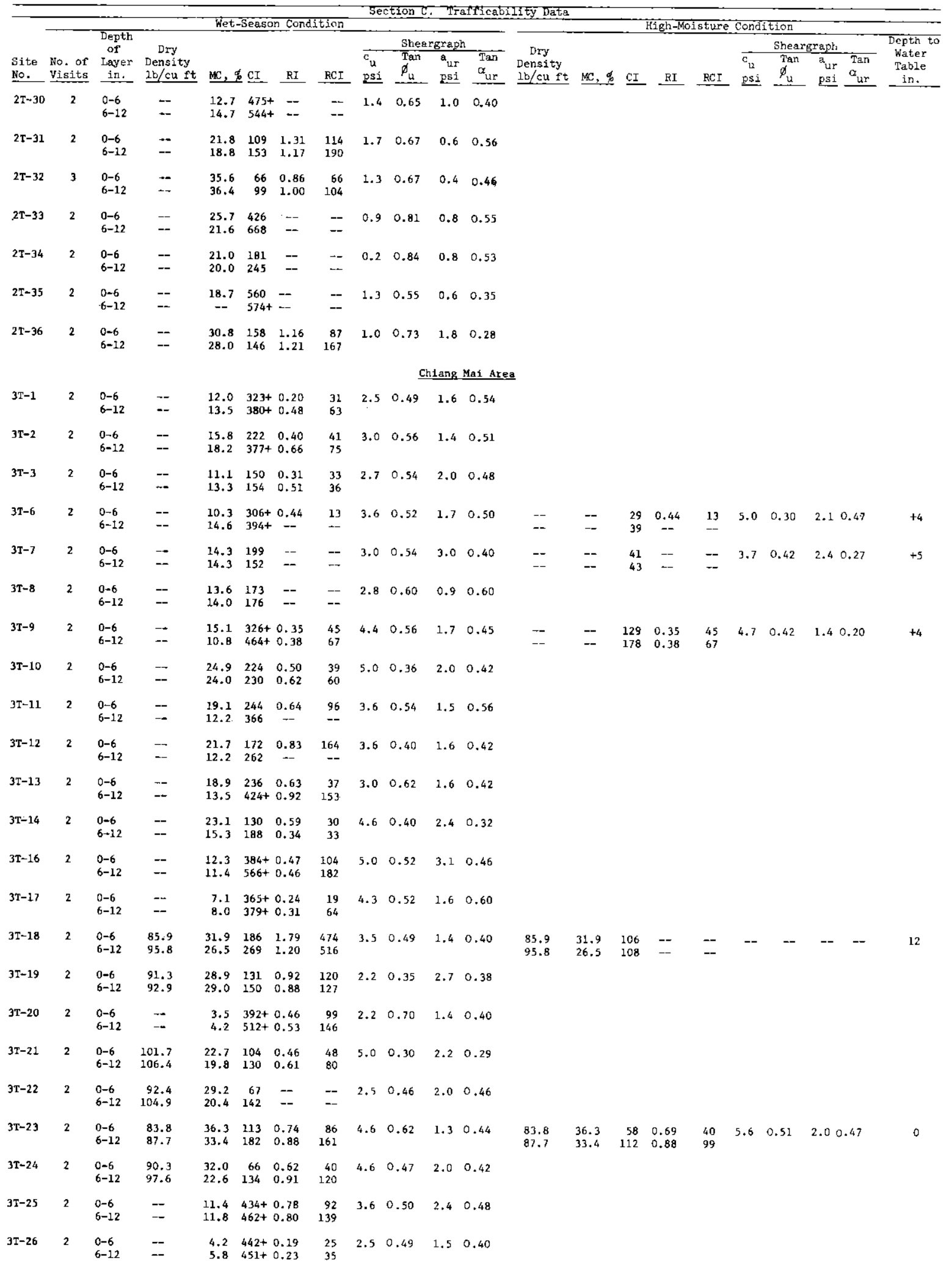




\begin{tabular}{|c|c|c|c|c|c|c|c|c|c|c|c|c|c|c|c|c|c|c|c|}
\hline \multirow[b]{2}{*}{$\begin{array}{l}\text { Site } \\
\text { No. }\end{array}$} & \multicolumn{2}{|c|}{ Location } & \multirow[b]{2}{*}{$\begin{array}{l}\text { Topog- } \\
\text { raphy } \\
\text { Class } \\
\end{array}$} & ection A & Site Da & ata & \multirow[b]{2}{*}{ Land Use } & \multirow[b]{2}{*}{$\begin{array}{l}\text { Depth } \\
\text { of } \\
\text { Layer } \\
\text { in. } \\
\end{array}$} & \multicolumn{9}{|c|}{$\begin{array}{r}\text { Section B. Soil Data } \\
\text { USCS }\end{array}$} & \multirow[b]{2}{*}{$\begin{array}{l}\text { Or- } \\
\text { ganic } \\
\text { Con- } \\
\text { tent } \\
\% \\
\end{array}$} & \multirow[b]{2}{*}{$\begin{array}{l}\text { Spe- } \\
\text { cific } \\
\text { Grav- } \\
\text { ity }\end{array}$} \\
\hline & $\begin{array}{c}\text { Map } \\
\text { Sheet } \\
\end{array}$ & $\begin{array}{l}\text { Gon } \\
\text { Grid } \\
\text { Coor- } \\
\text { di- } \\
\text { nates } \\
\end{array}$ & & $\begin{array}{l}\text { Topo- } \\
\text { graphic } \\
\text { Position } \\
\end{array}$ & $\begin{array}{c}\text { Slope } \\
\% \\
\end{array}$ & Yegetation & & & \multicolumn{3}{|c|}{$\begin{array}{l}-\frac{\text { USDA }}{\text { Texture }} \\
\text { by Wt, } \%\end{array}$} & Type & $\begin{array}{l}\text { By Wt } \\
\text { of } \\
\text { Fines } \\
\end{array}$ & $\begin{array}{r}\mathrm{At} \\
\mathrm{b} \\
\mathrm{L} \\
\mathrm{LL}\end{array}$ & $\begin{array}{l}\text { Atter- } \\
\text { bere }\end{array}$ & PI & Type & & \\
\hline $3 T-27$ & $4866 I V$ & 129456 & Low & $\begin{array}{l}\text { Bottomland } \\
\text { flat }\end{array}$ & 2 & Low scrub & Undisturbed & $\begin{array}{l}0-6 \\
6-12\end{array}$ & $\overline{33}$ & $\overline{44}$ & $\overline{23}$ & $\bar{L}$ & $\overline{76}$ & $\overline{31}$ & $\overline{20}$ & $\overline{11}$ & $\overline{\mathrm{CL}}$ & $\overline{1.49}$ & -- \\
\hline $3 T-28$ & 47661 & 973496 & Low & $\begin{array}{l}\text { Bot tomland } \\
\text { flat }\end{array}$ & 3 & $\begin{array}{l}\text { Short-grass } \\
\text { prafrie }\end{array}$ & $\begin{array}{l}\text { Cultivated } \\
\text { (grazed) }\end{array}$ & $\begin{array}{l}0-6 \\
6-12\end{array}$ & $\overline{25}$ & $\overline{48}$ & $\overline{27}$ & $\overline{\mathrm{L}}$ & $\overline{92}$ & $\overline{36}$ & $\overline{21}$ & $\overline{15}$ & $\overline{\mathrm{CL}}$ & $\overline{1.20}$ & -- \\
\hline $3 T-29$ & 47661 & 966472 & Low & $\begin{array}{l}\text { Bot tomland } \\
\text { f Iat }\end{array}$ & 1 & $\begin{array}{l}\text { Short-grass } \\
\text { prairie }\end{array}$ & $\begin{array}{l}\text { Cultivated } \\
\text { (grazed) }\end{array}$ & $\begin{array}{l}0-6 \\
6-12\end{array}$ & $\overline{60}$ & $\overline{24}$ & $\overline{16}$ & $\overline{\mathrm{SL}}$ & 52 & $\overline{23}$ & $\overline{16}$ & $-\overline{7}$ & $\overline{\mathrm{CL}-\mathrm{ML}}$ & 0.55 & -- \\
\hline $3 T-30$ & 47661 & 935475 & Low & $\begin{array}{l}\text { Bottomland } \\
\text { flat }\end{array}$ & 0 & $\begin{array}{l}\text { Short-grass } \\
\text { prairie }\end{array}$ & $\begin{array}{l}\text { Cu1tivated } \\
\text { (grazed) }\end{array}$ & $\begin{array}{l}0-6 \\
6-12\end{array}$ & $\overline{24}$ & $\overline{54}$ & $\overline{22}$ & siL & $\overline{85}$ & $\overline{38}$ & $\overline{25}$ & $\overline{13}$ & $\overline{\mathrm{ML}}$ & 2.23 & -- \\
\hline $3 T-31$ & $4766 \mathrm{I}$ & 959576 & Low & $\begin{array}{l}\text { Bottomland } \\
\text { flat }\end{array}$ & 1 & $\begin{array}{l}\text { Short-grass } \\
\text { prair1e }\end{array}$ & $\begin{array}{l}\text { Cultivated } \\
\text { (grazed) }\end{array}$ & $\begin{array}{l}0-6 \\
6-12\end{array}$ & 60 & $\overline{24}$ & $\overline{16}$ & $\overline{\mathrm{SL}}$ & $\overline{48}$ & $\overline{23}$ & $\overline{17}$ & $-\overline{6}$ & $\overline{S M-S C}$ & $\overline{1.00}$ & -- \\
\hline $3 T-32$ & 47661 & 993544 & Low & $\begin{array}{l}\text { Bot tomland } \\
\text { flat }\end{array}$ & 1 & $\begin{array}{l}\text { Short-grass } \\
\text { prafrie }\end{array}$ & $\begin{array}{l}\text { Culeivated } \\
\text { (1dle) }\end{array}$ & $\begin{array}{l}0-6 \\
6-12\end{array}$ & $\overline{23}$ & $\overline{41}$ & $\overline{36}$ & $\overline{\mathrm{CL}}$ & $\overline{84}$ & $\overline{37}$ & $\overline{25}$ & $\overline{12}$ & $\overline{M L}$ & 1.35 & -- \\
\hline & & & & & & & Pran Bur1 Area & & & & & & & & & & & & \\
\hline $4 \mathrm{~T}-2$ & 49471 & 994501 & Low & $\begin{array}{l}\text { Bot tomland } \\
\text { f lat }\end{array}$ & 0 & $\begin{array}{l}\text { Tal1-grass } \\
\text { pra1rie }\end{array}$ & Grazed & $\begin{array}{l}0-6 \\
6-12\end{array}$ & 17 & 39 & 44 & c & 98 & $\begin{array}{l}91 \\
--\end{array}$ & 36 & 55 & CH & $\begin{array}{c}4.34 \\
--\end{array}$ & $\ddot{-}$ \\
\hline $4 T-4$ & 49471 & 977463 & Low & $\begin{array}{l}\text { Botcomland } \\
\text { flat }\end{array}$ & 0 & $\begin{array}{l}\text { Tall-grass } \\
\text { pra1r1e }\end{array}$ & Grazed & $\begin{array}{l}0-6 \\
6-12\end{array}$ & $\begin{array}{l}13 \\
16\end{array}$ & $\begin{array}{l}62 \\
59\end{array}$ & $\begin{array}{l}25 \\
25\end{array}$ & $\begin{array}{l}\text { S1L } \\
\text { S1L }\end{array}$ & $\begin{array}{l}93 \\
93\end{array}$ & $\begin{array}{l}59 \\
46\end{array}$ & $\begin{array}{l}23 \\
18\end{array}$ & $\begin{array}{l}36 \\
28\end{array}$ & $\begin{array}{l}\mathrm{CH} \\
\mathrm{CL}\end{array}$ & $\begin{array}{l}1.45 \\
0.62\end{array}$ & -- \\
\hline $4 T-5$ & $4947 I$ & 982446 & Low & $\begin{array}{l}\text { Bottomland } \\
\text { flat }\end{array}$ & 0 & $\begin{array}{l}\text { Short-grass } \\
\text { pratrie }\end{array}$ & Grazed & $\begin{array}{l}0-6 \\
6-12\end{array}$ & $\overline{10}$ & $\overline{56}$ & $\overline{34}$ & $\overline{\mathrm{S} I \mathrm{CL}}$ & $\overline{96}$ & $\overline{57}$ & $\overline{20}$ & $\overline{37}$ & $\overline{\mathrm{CH}}$ & $\overline{1.98}$ & -- \\
\hline $4 T-6$ & $4947 I$ & 973451 & Low & $\begin{array}{l}\text { Bottomland } \\
\text { flat }\end{array}$ & 0 & $\begin{array}{l}\text { Tall-grass } \\
\text { prafrie }\end{array}$ & Grazed & $\begin{array}{l}0-6 \\
6-12\end{array}$ & $\begin{array}{l}20 \\
20\end{array}$ & $\begin{array}{l}45 \\
45\end{array}$ & $\begin{array}{l}35 \\
35\end{array}$ & $\begin{array}{l}\text { SiCL } \\
\text { SiCL }\end{array}$ & $\begin{array}{l}99 \\
99\end{array}$ & $\begin{array}{l}50 \\
50\end{array}$ & $\begin{array}{l}17 \\
17\end{array}$ & $\begin{array}{l}33 \\
33\end{array}$ & $\begin{array}{l}\mathrm{CH} \\
\mathrm{CH}\end{array}$ & $\begin{array}{l}1.15 \\
1.15\end{array}$ & -- \\
\hline $4 T-7$ & 494711 & 970446 & Low & $\begin{array}{l}\text { Bottomland } \\
\text { flat }\end{array}$ & 0 & $\begin{array}{l}\text { Tal1-grass } \\
\text { pra1rie }\end{array}$ & Grazed & $\begin{array}{l}0-6 \\
6-12\end{array}$ & $-\overline{9}$ & $\overline{49}$ & $\overline{42}$ & sic & $\overline{97}$ & $\overline{64}$ & $\overline{25}$ & $\overline{39}$ & $\overline{\mathrm{CH}}$ & 3.54 & -- \\
\hline $4 T-10$ & 494711 & 984425 & Low & $\begin{array}{l}\text { Bottomland } \\
\text { flat }\end{array}$ & 0 & $\begin{array}{l}\text { Tal1-grass } \\
\text { pra1rie }\end{array}$ & Grazed & $\begin{array}{l}0-6 \\
6-12\end{array}$ & $\begin{array}{l}7 \\
7\end{array}$ & $\begin{array}{l}51 \\
51\end{array}$ & $\begin{array}{l}42 \\
42\end{array}$ & $\begin{array}{l}\text { SiC } \\
\text { SiC }\end{array}$ & $\begin{array}{l}95 \\
95\end{array}$ & $\begin{array}{l}76 \\
76\end{array}$ & $\begin{array}{l}28 \\
28\end{array}$ & $\begin{array}{l}48 \\
48\end{array}$ & $\begin{array}{l}\mathrm{CH} \\
\mathrm{CH}\end{array}$ & $\begin{array}{l}0.55 \\
0.55\end{array}$ & $\cdots$ \\
\hline $4 \mathrm{~T}-11$ & $4947 I$ & 012526 & Low & $\begin{array}{l}\text { Bottomland } \\
\text { flat }\end{array}$ & 0 & $\begin{array}{l}\text { Short-grass } \\
\text { pralrie }\end{array}$ & Grazed & $\begin{array}{l}0-6 \\
6-12\end{array}$ & $\begin{array}{l}17 \\
32\end{array}$ & $\begin{array}{l}32 \\
35\end{array}$ & $\begin{array}{l}51 \\
33\end{array}$ & $\begin{array}{l}\mathrm{C} \\
\mathrm{CL}\end{array}$ & $\begin{array}{l}93 \\
74\end{array}$ & $\begin{array}{l}70 \\
60\end{array}$ & $\begin{array}{l}36 \\
24\end{array}$ & $\begin{array}{l}34 \\
36\end{array}$ & $\underset{\mathrm{CH}}{\mathrm{MH}}$ & $\begin{array}{l}5.24 \\
3.41\end{array}$ & -- \\
\hline $4 T-12$ & $4947 \mathrm{I}$ & 018543 & Low & $\begin{array}{l}\text { Bottomland } \\
\text { f1at }\end{array}$ & 0 & $\begin{array}{l}\text { Short-grass } \\
\text { pralrie }\end{array}$ & Grazed & $\begin{array}{l}0-6 \\
6-12\end{array}$ & $\begin{array}{l}30 \\
41\end{array}$ & $\begin{array}{l}27 \\
31\end{array}$ & $\begin{array}{l}43 \\
28\end{array}$ & $\begin{array}{l}\mathrm{C} \\
\mathrm{CL}\end{array}$ & $\begin{array}{l}79 \\
64\end{array}$ & $\begin{array}{l}70 \\
47\end{array}$ & $\begin{array}{l}32 \\
19\end{array}$ & $\begin{array}{l}38 \\
28\end{array}$ & $\begin{array}{l}\mathrm{CH} \\
\mathrm{CL}\end{array}$ & $\begin{array}{l}5.24 \\
3.13\end{array}$ & $\overline{--}$ \\
\hline $4 \mathrm{~T}-20$ & 4948II & 901741 & Low & $\begin{array}{l}\text { Bot tomland } \\
\text { flat }\end{array}$ & 0 & $\begin{array}{l}\text { Short-grass } \\
\text { pratrte }\end{array}$ & $\begin{array}{l}\text { Cultivated } \\
\text { (sugar cane) }\end{array}$ & $\begin{array}{l}0-6 \\
6-12\end{array}$ & $\begin{array}{r}8 \\
--\end{array}$ & $\begin{array}{l}69 \\
--\end{array}$ & 23 & SiL & $\begin{array}{l}99 \\
--\end{array}$ & $\begin{array}{l}34 \\
--\end{array}$ & 21 & $\begin{array}{l}13 \\
--\end{array}$ & $\mathrm{CL}$ & 1.33 & $\overline{-}$ \\
\hline $4 T-21$ & $4948 I I$ & 904778 & Low & $\begin{array}{l}\text { Bottomland } \\
\text { flat }\end{array}$ & 0 & $\begin{array}{l}\text { Tall scrub } \\
\text { savanna }\end{array}$ & Undisturbed & $\begin{array}{l}0-6 \\
6-12\end{array}$ & $\begin{array}{l}60 \\
56\end{array}$ & $\begin{array}{l}26 \\
25\end{array}$ & $\begin{array}{l}14 \\
19\end{array}$ & $\begin{array}{l}\text { SL } \\
\text { SL }\end{array}$ & $\begin{array}{l}48 \\
52\end{array}$ & $\begin{array}{l}15 \\
17\end{array}$ & $\begin{array}{l}14 \\
12\end{array}$ & $\begin{array}{l}1 \\
5\end{array}$ & $\underset{\mathrm{CL}-\mathrm{ML}}{\mathrm{SM}}$ & $\begin{array}{l}0.78 \\
0.70\end{array}$ & -- \\
\hline $4 \mathrm{~T}-22$ & $4948 \mathrm{II}$ & 898791 & Low & $\begin{array}{l}\text { Bottonland } \\
\text { f lat }\end{array}$ & 0 & $\begin{array}{l}\text { Tall scrub } \\
\text { forest }\end{array}$ & Und1sturbed & $\begin{array}{l}0-6 \\
6-12\end{array}$ & $\begin{array}{l}24 \\
--\end{array}$ & $\begin{array}{l}56 \\
--\end{array}$ & 20 & $\begin{array}{l}\text { S1L } \\
--\end{array}$ & $\begin{array}{l}83 \\
--\end{array}$ & $\stackrel{34}{--}$ & 23 & 11 & $\begin{array}{l}\text { CL } \\
--\end{array}$ & 3.13 & $\overrightarrow{-}$ \\
\hline $4 T-23$ & $49481 I$ & 879752 & Low & $\begin{array}{l}\text { Bot tomland } \\
\text { flat }\end{array}$ & 0 & Forest & Und1sturbed & $\begin{array}{l}0-6 \\
6-12\end{array}$ & 58 & $\begin{array}{l}29 \\
--\end{array}$ & 13 & $\begin{array}{l}\text { SL } \\
--\end{array}$ & 55 & $\begin{array}{l}14 \\
--\end{array}$ & 14 & -0 & MI & 0.70 & -- \\
\hline $4 T-24$ & $49481 I$ & 862776 & Low & $\begin{array}{l}\text { Bottomland } \\
\text { flat }\end{array}$ & 0 & Forest & Undisturbed & $\begin{array}{l}0-6 \\
6-12\end{array}$ & $\begin{array}{l}58 \\
00\end{array}$ & $\begin{array}{l}29 \\
27\end{array}$ & $\begin{array}{l}13 \\
13\end{array}$ & $\begin{array}{l}\text { SL } \\
\text { SL }\end{array}$ & $\begin{array}{l}50 \\
48\end{array}$ & $\begin{array}{l}22 \\
17\end{array}$ & $\begin{array}{l}18 \\
14\end{array}$ & $\begin{array}{l}4 \\
3\end{array}$ & $\begin{array}{l}\text { SM } \\
\text { SM }\end{array}$ & $\begin{array}{l}2.75 \\
1.33\end{array}$ & -- \\
\hline $4 T-25$ & $4948 \mathrm{II}$ & 837739 & Low & $\begin{array}{l}\text { Natura1 } \\
\text { levee }\end{array}$ & 0 & Forest & Undisturbed & $\begin{array}{l}0-6 \\
6-12\end{array}$ & $\overline{35}$ & $\overrightarrow{41}$ & $\overline{24}$ & $\bar{L}$ & $\overline{77}$ & $\overline{24}$ & $\overline{16}$ & $\overline{8}$ & $\overline{\mathrm{CL}}$ & $\overline{0.95}$ & -- \\
\hline $4 T-26$ & $4948 \mathrm{II}$ & 845745 & Low & $\begin{array}{l}\text { Bot tomland } \\
\text { flat }\end{array}$ & 0 & Forest & Undisturbed & $\begin{array}{l}0-6 \\
6-12\end{array}$ & $\begin{array}{l}48 \\
44\end{array}$ & $\begin{array}{l}31 \\
25\end{array}$ & $\begin{array}{l}21 \\
31\end{array}$ & $\underset{\text { GCL }}{\mathrm{L}}$ & $\begin{array}{l}60 \\
40\end{array}$ & $\begin{array}{l}26 \\
30\end{array}$ & $\begin{array}{l}14 \\
14\end{array}$ & $\begin{array}{l}12 \\
16\end{array}$ & $\begin{array}{l}\mathrm{CL} \\
\mathrm{SC}\end{array}$ & $\begin{array}{l}1.15 \\
0.86\end{array}$ & -- \\
\hline $4 T-27$ & $4947 \mathrm{I}$ & 056578 & Low & $\begin{array}{l}\text { Bottomland } \\
\text { depression }\end{array}$ & 0 & Barren & Und1sturbed & $\begin{array}{l}0-6 \\
6-12\end{array}$ & $\begin{array}{l}69 \\
39\end{array}$ & $\begin{array}{l}16 \\
20\end{array}$ & $\begin{array}{l}15 \\
41\end{array}$ & $\begin{array}{l}\text { SL } \\
\text { C }\end{array}$ & $\begin{array}{l}38 \\
66\end{array}$ & $\begin{array}{l}17 \\
45\end{array}$ & $\begin{array}{l}14 \\
15\end{array}$ & $\begin{array}{r}3 \\
30\end{array}$ & $\begin{array}{l}\mathrm{SM} \\
\mathrm{CL}\end{array}$ & $\begin{array}{l}0.46 \\
0.55\end{array}$ & -- \\
\hline $4 \mathrm{~T}-28$ & 49471 & 054562 & Lav & $\begin{array}{l}\text { Bot tomland } \\
\text { f lat }\end{array}$ & 0 & $\begin{array}{l}\text { Short-grass } \\
\text { pralrie }\end{array}$ & $\begin{array}{l}\text { Cultivated } \\
\text { (grazed) }\end{array}$ & $\begin{array}{l}0-6 \\
6-12\end{array}$ & $\begin{array}{l}27 \\
17\end{array}$ & $\begin{array}{l}50 \\
51\end{array}$ & $\begin{array}{l}23 \\
32\end{array}$ & $\begin{array}{l}\text { S1L } \\
\text { S1CL }\end{array}$ & $\begin{array}{l}87 \\
93\end{array}$ & $\begin{array}{l}37 \\
37\end{array}$ & $\begin{array}{l}20 \\
20\end{array}$ & $\begin{array}{l}17 \\
17\end{array}$ & $\begin{array}{l}\mathrm{CL} \\
\mathrm{CL}\end{array}$ & $\begin{array}{l}3.62 \\
1.98\end{array}$ & 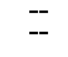 \\
\hline $4 T-29$ & $4947 I$ & 986530 & Low & $\begin{array}{l}\text { Bot tomland } \\
\text { flat }\end{array}$ & 0 & $\begin{array}{l}\text { Short-grass } \\
\text { prafrie }\end{array}$ & Grazed & $\begin{array}{l}0-6 \\
6-12\end{array}$ & $\begin{array}{l}11 \\
24\end{array}$ & $\begin{array}{l}66 \\
52\end{array}$ & $\begin{array}{l}23 \\
24\end{array}$ & $\begin{array}{l}\text { S1L } \\
\text { SiL }\end{array}$ & $\begin{array}{l}99 \\
86\end{array}$ & $\begin{array}{l}34 \\
26\end{array}$ & $\begin{array}{l}22 \\
16\end{array}$ & $\begin{array}{l}12 \\
10\end{array}$ & $\begin{array}{l}\mathrm{CL} \\
\mathrm{CL}\end{array}$ & $\begin{array}{l}1.33 \\
1.45\end{array}$ & -- \\
\hline $4 \pi-31$ & $4948 \mathrm{II}$ & 929750 & Lav & $\begin{array}{l}\text { Bot tomland } \\
\text { flat }\end{array}$ & 0 & $\begin{array}{l}\text { Tall scrub } \\
\text { forest }\end{array}$ & Undisturbed & $\begin{array}{l}0-6 \\
6-12\end{array}$ & $\begin{array}{l}63 \\
57\end{array}$ & $\begin{array}{l}24 \\
17\end{array}$ & $\begin{array}{l}13 \\
26\end{array}$ & $\begin{array}{r}\text { SL } \\
\text { SCL }\end{array}$ & $\begin{array}{l}50 \\
50\end{array}$ & $\begin{array}{l}16 \\
22\end{array}$ & $\begin{array}{l}13 \\
10\end{array}$ & $\begin{array}{r}3 \\
12\end{array}$ & $\begin{array}{l}S M \\
S C\end{array}$ & $\begin{array}{l}0.95 \\
0.70\end{array}$ & -- \\
\hline $4 T-32$ & $4948 \mathrm{II}$ & 918744 & Low & $\begin{array}{l}\text { Bot tomland } \\
\text { flat }\end{array}$ & 0 & $\begin{array}{l}\text { Short-grass } \\
\text { prair1e }\end{array}$ & $\begin{array}{l}\text { Cultivated } \\
\text { (rice) }\end{array}$ & $\begin{array}{l}0-6 \\
6-12\end{array}$ & $\begin{array}{l}20 \\
21\end{array}$ & $\begin{array}{l}52 \\
43\end{array}$ & $\begin{array}{l}28 \\
36\end{array}$ & $\begin{array}{c}\text { S1CL } \\
\text { CL }\end{array}$ & $\begin{array}{l}88 \\
87\end{array}$ & $\begin{array}{l}31 \\
35\end{array}$ & $\begin{array}{l}14 \\
13\end{array}$ & $\begin{array}{l}17 \\
22\end{array}$ & $\begin{array}{l}\text { CL } \\
\text { CL }\end{array}$ & $\begin{array}{l}1.25 \\
0.55\end{array}$ & $\ddot{m}$ \\
\hline $4 T-33$ & $4948 \mathrm{II}$ & 908723 & Low & $\begin{array}{l}\text { Bot tomland } \\
\text { f lat }\end{array}$ & 0 & $\begin{array}{l}\text { Short-grass } \\
\text { pralrie }\end{array}$ & $\begin{array}{l}\text { Cultivated } \\
\text { (sugar cane) }\end{array}$ & $\begin{array}{l}0-6 \\
6-12\end{array}$ & $\begin{array}{l}22 \\
20\end{array}$ & $\begin{array}{l}53 \\
55\end{array}$ & $\begin{array}{l}25 \\
25\end{array}$ & $\begin{array}{l}\mathrm{S} 1 \mathrm{~L} \\
\mathrm{~S} 1 \mathrm{~L}\end{array}$ & $\begin{array}{l}87 \\
91\end{array}$ & $\begin{array}{l}30 \\
32\end{array}$ & $\begin{array}{l}18 \\
19\end{array}$ & $\begin{array}{l}12 \\
13\end{array}$ & $\begin{array}{l}\text { CL } \\
\text { CL }\end{array}$ & $\begin{array}{l}1.15 \\
1.15\end{array}$ & 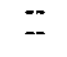 \\
\hline & & & & & & & on Kaen Area & & & & & & & & & & & & \\
\hline $5 \mathrm{~T}-1$ & $5560 \mathrm{II}$ & 733172 & LoH & $\begin{array}{l}\text { got tonland } \\
\text { flat }\end{array}$ & 0 & $\begin{array}{l}\text { Short-grass } \\
\text { pralrie }\end{array}$ & $\begin{array}{l}\text { Cultivated } \\
\text { (rice) }\end{array}$ & $\begin{array}{l}0-6 \\
6-12\end{array}$ & $\begin{array}{l}30 \\
34\end{array}$ & $\begin{array}{l}58 \\
56\end{array}$ & $\begin{array}{l}12 \\
10\end{array}$ & $\begin{array}{l}\text { SiL } \\
\text { S1L }\end{array}$ & $\begin{array}{l}81 \\
77\end{array}$ & $\begin{array}{l}28 \\
28\end{array}$ & $\begin{array}{l}17 \\
17\end{array}$ & $\begin{array}{l}11 \\
11\end{array}$ & $\begin{array}{l}\mathrm{CL} \\
\mathrm{CL}\end{array}$ & $\begin{array}{l}0.78 \\
0.55\end{array}$ & $\begin{array}{l}2.67 \\
2.69\end{array}$ \\
\hline $5 T-2$ & $55601 \mathrm{I}$ & 766184 & Low & $\begin{array}{l}\text { Bottomland } \\
\text { flat }\end{array}$ & 0 & $\begin{array}{l}\text { Short-graes } \\
\text { prairie }\end{array}$ & $\begin{array}{l}\text { Cult fvated } \\
\text { (r1ce) }\end{array}$ & $\begin{array}{l}0-6 \\
6-12\end{array}$ & $\begin{array}{l}16 \\
14\end{array}$ & $\begin{array}{l}60 \\
52\end{array}$ & $\begin{array}{l}24 \\
34\end{array}$ & $\begin{array}{l}\text { S1L } \\
\text { S1CL }\end{array}$ & $\begin{array}{l}88 \\
91\end{array}$ & $\begin{array}{l}68 \\
79\end{array}$ & $\begin{array}{l}40 \\
46\end{array}$ & $\begin{array}{l}28 \\
33\end{array}$ & $\begin{array}{l}\text { MH } \\
\text { MH }\end{array}$ & $\begin{array}{l}1.15 \\
1.05\end{array}$ & $\begin{array}{l}2.70 \\
2.78\end{array}$ \\
\hline
\end{tabular}




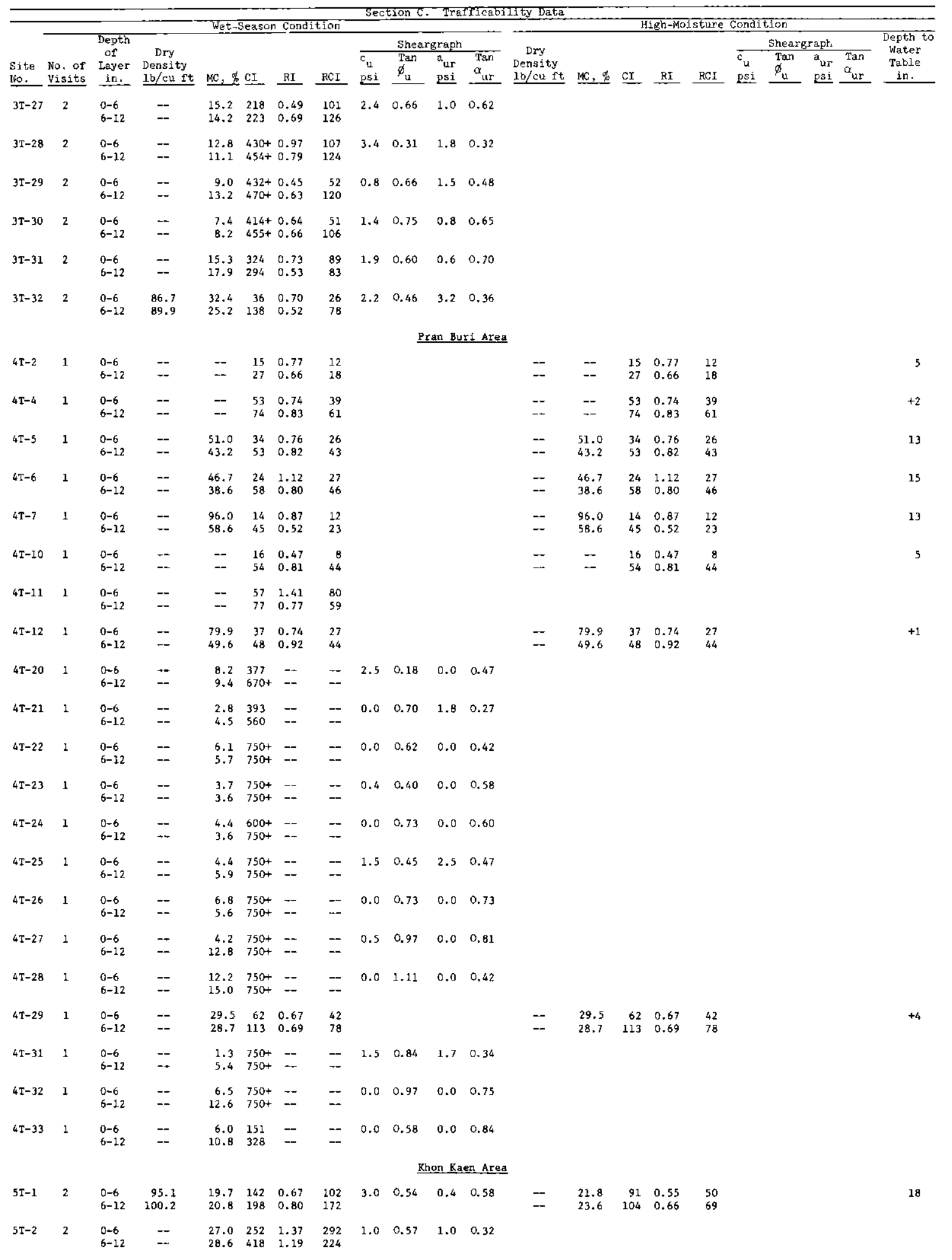




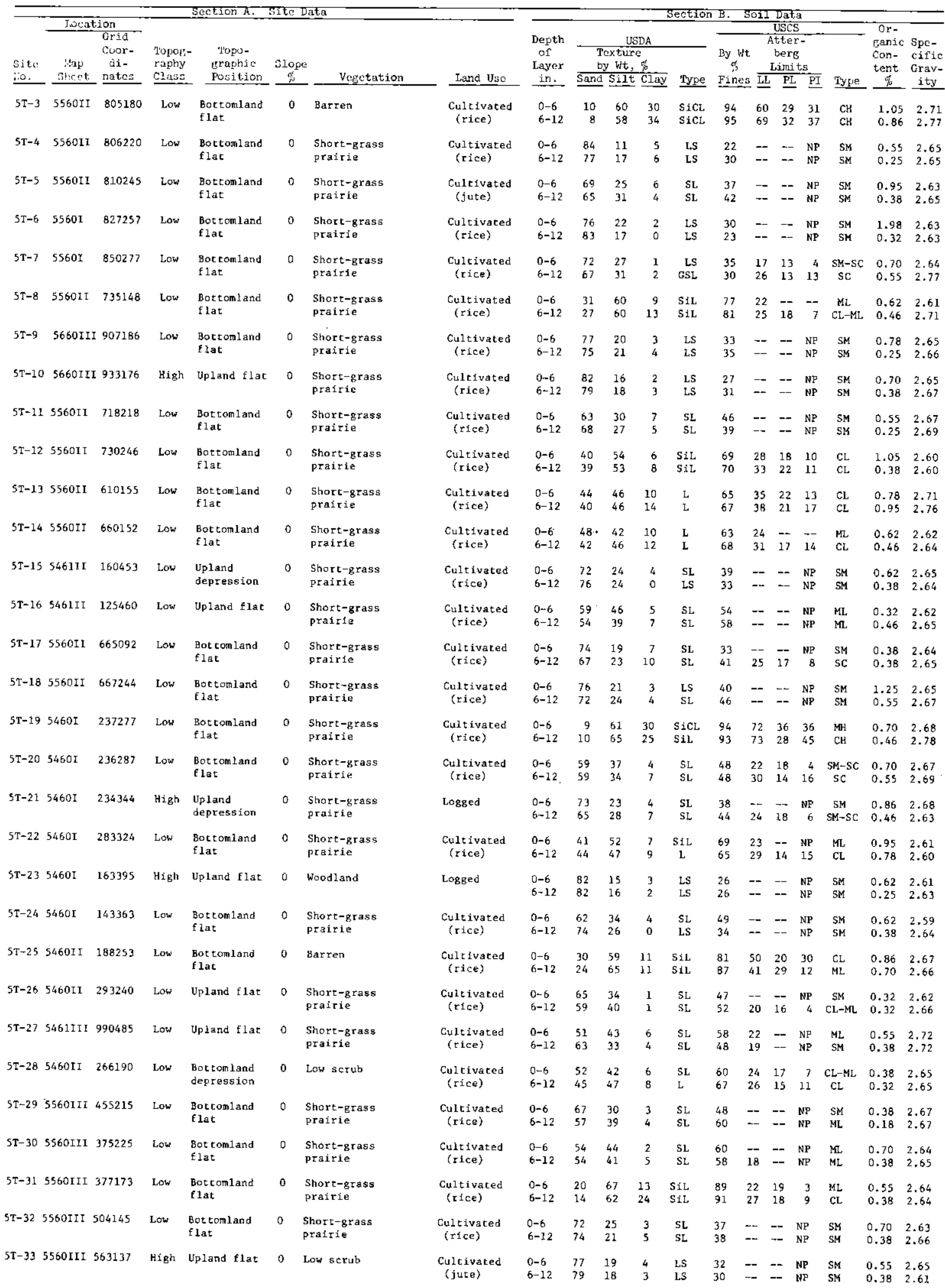




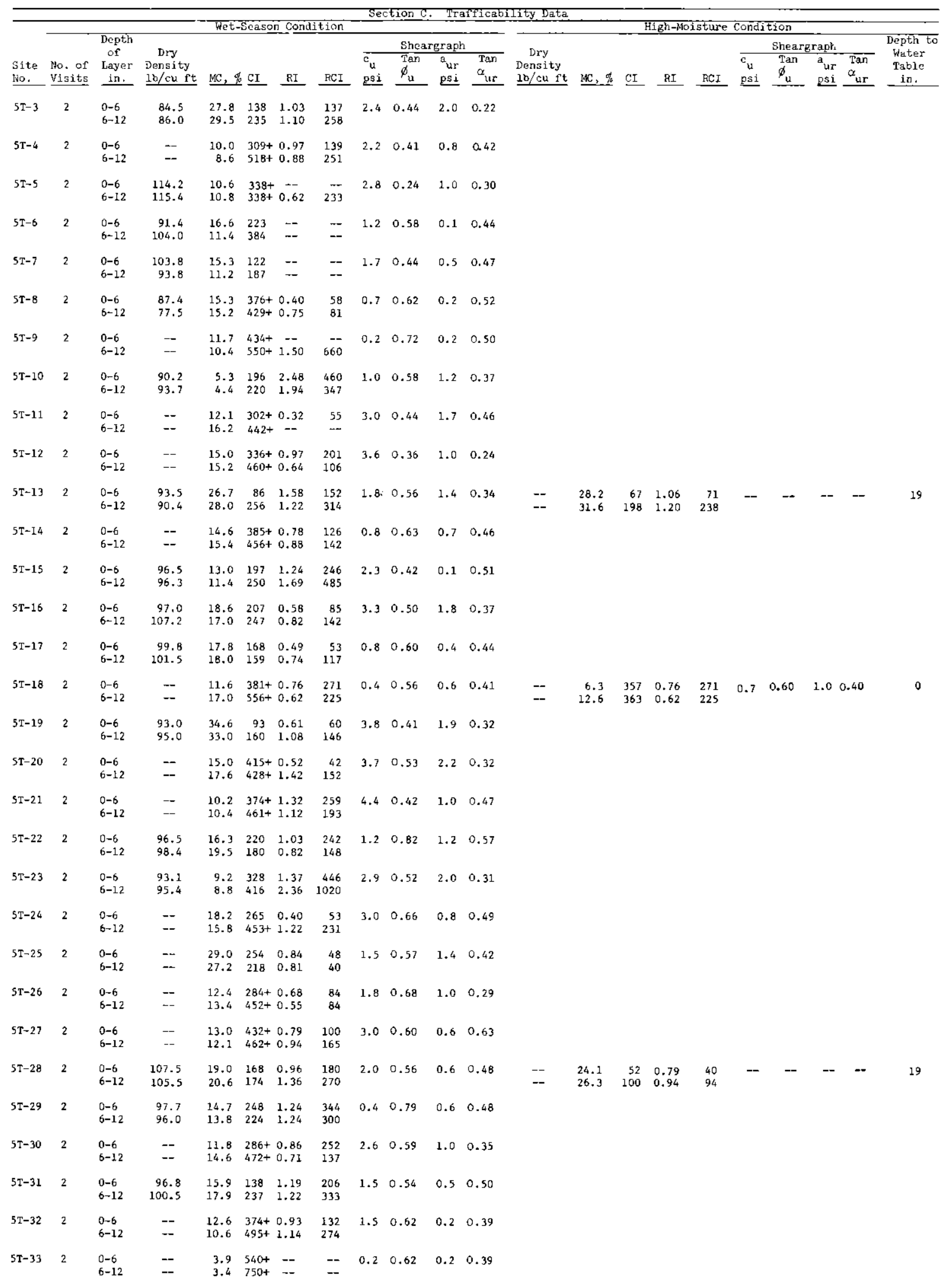




\begin{tabular}{|c|c|c|c|c|c|c|c|c|c|c|c|c|c|c|c|c|c|c|c|}
\hline \multirow[b]{3}{*}{$\begin{array}{l}\text { Site } \\
\text { No. } \\
\end{array}$} & \multirow{2}{*}{\multicolumn{2}{|c|}{ Location }} & \multirow[b]{3}{*}{$\begin{array}{l}\text { Topog- } \\
\text { raphy } \\
\text { Class } \\
\end{array}$} & Section $A$. & \multirow[b]{3}{*}{$\begin{array}{c}\text { slope } \\
\% \\
\end{array}$} & ata & \multirow[b]{3}{*}{ Land Use } & \multirow[b]{3}{*}{$\begin{array}{c}\text { Depth } \\
\text { of } \\
\text { Layer } \\
\text { in. } \\
\end{array}$} & \multirow{2}{*}{\multicolumn{9}{|c|}{ Section B. Soil Data }} & \multirow[b]{3}{*}{$\begin{array}{l}\text { Or- } \\
\text { ganic } \\
\text { Con- } \\
\text { tent } \\
\% \\
\end{array}$} & \multirow[b]{3}{*}{$\begin{array}{l}\text { Spe- } \\
\text { cific } \\
\text { Grav- } \\
\text { ity } \\
\end{array}$} \\
\hline & & & & \multirow[b]{2}{*}{$\begin{array}{l}\text { Topo- } \\
\text { Eraphic } \\
\text { Position } \\
\end{array}$} & & \multirow[b]{2}{*}{ Vegetation } & & & & & & & & & & & & & \\
\hline & $\begin{array}{c}\text { Map } \\
\text { Sheet }\end{array}$ & $\begin{array}{c}\text { coor- } \\
\text { di- } \\
\text { nates }\end{array}$ & & & & & & & \multicolumn{3}{|c|}{$\begin{array}{l}\text { USDA } \\
\text { Texture } \\
\text { by Wt, } \%\end{array}$} & Tyge & \multicolumn{4}{|c|}{$\begin{array}{l}\begin{array}{c}\text { Atter- } \\
\text { berg } \\
\text { Limits }\end{array} \\
\text { L PL PI }\end{array}$} & Tyge & & \\
\hline $5 T-34$ & S560III & 515237 & Low & $\begin{array}{l}\text { Bottom: and } \\
\text { flat }\end{array}$ & 0 & Barren & $\begin{array}{l}\text { Cult 1vated } \\
\text { (r1ce) }\end{array}$ & $\begin{array}{l}0-6 \\
6-12\end{array}$ & $\begin{array}{l}56 \\
50\end{array}$ & $\begin{array}{l}41 \\
44\end{array}$ & $\begin{array}{l}3 \\
6\end{array}$ & $\begin{array}{l}\text { SL } \\
\text { SL }\end{array}$ & $\begin{array}{l}52 \\
52\end{array}$ & $\overline{17}$ & $\overline{13}$ & $\begin{array}{r}\mathrm{NP} \\
4\end{array}$ & $\underset{C L-M L}{M L}$ & $\begin{array}{l}0.32 \\
0.32\end{array}$ & $\begin{array}{l}2.60 \\
2.63\end{array}$ \\
\hline $5 T-35$ & $5560 \mathrm{III}$ & 560207 & H1gh & Upland flat & 0 & $\begin{array}{l}\text { Short-grass } \\
\text { pralrle }\end{array}$ & $\begin{array}{l}\text { Cultivated } \\
\text { (jute) }\end{array}$ & $\begin{array}{l}0-6 \\
6-12\end{array}$ & $\begin{array}{l}76 \\
75\end{array}$ & $\begin{array}{l}21 \\
22\end{array}$ & $\begin{array}{l}3 \\
3\end{array}$ & $\begin{array}{l}\text { LS } \\
\text { LS }\end{array}$ & $\begin{array}{l}33 \\
34\end{array}$ & -- & $\overline{--}$ & $\begin{array}{l}\mathrm{NP} \\
\mathrm{NP}\end{array}$ & $\begin{array}{l}\text { SM } \\
\text { SM }\end{array}$ & $\begin{array}{l}1.05 \\
0.86\end{array}$ & $\begin{array}{l}2.62 \\
2.65\end{array}$ \\
\hline $5 T-40$ & $5560 \mathrm{II}$ & 797160 & Low & $\begin{array}{l}\text { Bot tomland } \\
\text { f lat }\end{array}$ & 0 & Low scrub & Logged & $\begin{array}{l}0-6 \\
6-12\end{array}$ & $-\overline{7}$ & $\overline{28}$ & -5 & $\bar{c}$ & $\overline{96}$ & $\ddot{86}$ & $-\overline{30}$ & $\overline{56}$ & $\overline{\mathrm{CH}}$ & $\begin{array}{l}4.70 \\
2.87\end{array}$ & $\overline{-}$ \\
\hline $5 T-41$ & $5560 \mathrm{II}$ & 792158 & Low & $\begin{array}{l}\text { Bottomland } \\
\text { depression }\end{array}$ & 0 & Low scrub & Logged & $\begin{array}{l}0-6 \\
6-12\end{array}$ & -- & 35 & $\overline{60}$ & $\overline{\mathrm{c}}$ & 97 & 80 & $\overline{28}$ & $\overline{52}$ & $\overline{\mathrm{CH}}$ & $\begin{array}{l}3.96 \\
1.98\end{array}$ & $\begin{array}{l}-- \\
--\end{array}$ \\
\hline $5 T-42$ & $5560 \mathrm{II}$ & 794157 & Low & $\begin{array}{l}\text { Bottom1and } \\
\text { flat }\end{array}$ & 0 & $\begin{array}{l}\text { Tall-grass } \\
\text { pra1rle }\end{array}$ & Undisturbed & $\begin{array}{l}0-6 \\
6-12\end{array}$ & $-\overline{6}$ & $\overline{23}$ & $\overline{71}$ & c- & 97 & $\overline{100}$ & $\overline{31}$ & -- & $\overrightarrow{\mathrm{CH}}$ & $\begin{array}{l}2.23 \\
1.98\end{array}$ & -- \\
\hline $5 T-43$ & $5560 \mathrm{II}$ & 794153 & Low & $\begin{array}{l}\text { Bottomland } \\
\text { f lat }\end{array}$ & 0 & Low scrub & Undisturbed & $\begin{array}{l}0-6 \\
6-12\end{array}$ & $\overline{6}$ & $-\overline{28}$ & $\overline{66}$ & $\bar{c}$ & -- & $\overline{79}$ & $\overline{27}$ & $\overline{52}$ & $\overline{\mathrm{CH}}$ & $\begin{array}{l}5.74 \\
3.96\end{array}$ & -- \\
\hline $5 T-44$ & 556011 & 794153 & Low & $\begin{array}{l}\text { Bottomland } \\
\text { depression }\end{array}$ & 0 & Low scrub & Logged & $\begin{array}{l}0-6 \\
6-12\end{array}$ & $-\overline{7}$ & 29 & $\overline{64}$ & $\bar{c}$ & 97 & 93 & $\overline{38}$ & $-\overline{5}$ & $\overline{\mathrm{CH}}$ & $\begin{array}{l}5.50 \\
2.75\end{array}$ & - \\
\hline $5 T-45$ & $5560 I 1$ & 794153 & Low & $\begin{array}{l}\text { Bottomland } \\
\text { depression }\end{array}$ & 0 & Low scrub & Logged & $\begin{array}{l}0-6 \\
6-12\end{array}$ & $-\overline{5}$ & $\overline{27}$ & $-\overline{68}$ & $\bar{c}$ & $\overline{99}$ & -- & $\begin{array}{l}-- \\
35\end{array}$ & $\overline{58}$ & $\overline{\mathrm{CH}}$ & $\begin{array}{l}7.24 \\
3.41\end{array}$ & - \\
\hline $5 T-46$ & 556011 & 727240 & Low & $\begin{array}{l}\text { Bottomland } \\
\text { f lat }\end{array}$ & 0 & $\begin{array}{l}\text { Short grass } \\
\text { prairie }\end{array}$ & $\begin{array}{l}\text { CuItivated } \\
\text { (rice) }\end{array}$ & $\begin{array}{l}0-6 \\
6-12\end{array}$ & -2 & $\overline{26}$ & $\overline{16}$ & $\overline{S L}$ & $\overline{53}$ & $\overline{21}$ & $\overline{12}$ & $\bar{g}$ & $\overline{\mathrm{CL}}$ & $\begin{array}{l}1.25 \\
0.70\end{array}$ & -- \\
\hline $5 T-47$ & 556011 & 727237 & Low & $\begin{array}{l}\text { Bottomland } \\
\text { flat }\end{array}$ & 0 & $\begin{array}{l}\text { Short-grass } \\
\text { prairte }\end{array}$ & $\begin{array}{l}\text { Cultivated } \\
\text { (rice) }\end{array}$ & $\begin{array}{l}0-6 \\
6-12\end{array}$ & $\overline{33}$ & 38 & $\overline{29}$ & $\overline{\mathrm{CL}}$ & 76 & $\overline{32}$ & $\overline{13}$ & -- & $\overline{C L}$ & $\begin{array}{l}1.45 \\
0.95\end{array}$ & -- \\
\hline $5 T-48$ & $5560 \mathrm{II}$ & 727240 & Low & $\begin{array}{l}\text { Bottom Iand } \\
\text { flat }\end{array}$ & 0 & $\begin{array}{l}\text { Short-grass } \\
\text { prairie }\end{array}$ & $\begin{array}{l}\text { Cultivated } \\
\text { (rice) }\end{array}$ & $\begin{array}{l}0-6 \\
6-12\end{array}$ & $\overline{35}$ & 41 & $\overline{24}$ & $\bar{L}$ & $\overline{76}$ & $\overline{29}$ & $\overline{13}$ & $\overline{16}$ & $\overrightarrow{\mathrm{CL}}$ & $\begin{array}{l}0.78 \\
0.70\end{array}$ & $=$ \\
\hline $5 T-49$ & $5560 \mathrm{TI}$ & 732237 & Low & $\begin{array}{l}\text { Bottomland } \\
\text { flat }\end{array}$ & 0 & $\begin{array}{l}\text { Short-grass } \\
\text { prairie }\end{array}$ & $\begin{array}{l}\text { Cultivated } \\
\text { (rice) }\end{array}$ & $\begin{array}{l}0-6 \\
6-12\end{array}$ & $\overline{35}$ & $\overline{27}$ & $\overline{38}$ & $\overline{\mathrm{CL}}$ & $\overline{74}$ & $\frac{-}{40}$ & $\overline{14}$ & $\overline{26}$ & $\overline{\mathrm{CL}}$ & $\begin{array}{l}1.25 \\
0.86\end{array}$ & -- \\
\hline $5 T-50$ & $5560 \mathrm{II}$ & 760201 & Low & $\begin{array}{l}\text { Bottomland } \\
\text { flat }\end{array}$ & 0 & $\begin{array}{l}\text { Short-grass } \\
\text { prairie }\end{array}$ & $\begin{array}{l}\text { Cultivated } \\
\text { (rice) }\end{array}$ & $\begin{array}{l}0-6 \\
6-12\end{array}$ & $\overline{27}$ & $\overline{53}$ & $\overline{20}$ & $\overline{\text { SIL }}$ & $\overline{89}$ & $\overline{28}$ & $\overline{18}$ & $\overline{10}$ & $\ddot{C L}$ & $\begin{array}{l}1.15 \\
0.78\end{array}$ & -- \\
\hline & & & & & & & habur: Area & & & & & & & & & & & & \\
\hline $6 \mathrm{~T}-1$ & $5349 \mathrm{III}$ & 486103 & Low & $\begin{array}{l}\text { Bot tomland } \\
\text { flat }\end{array}$ & 0 & Woodland & $\begin{array}{l}\text { Rubber } \\
\text { plantation }\end{array}$ & $\begin{array}{l}0-6 \\
6-12\end{array}$ & $\overline{52}$ & $\overline{30}$ & $\overline{18}$ & $\overline{\mathrm{GL}}$ & $\overline{29}$ & $\overline{48}$ & 30 & $\overline{18}$ & $\overline{S M}$ & 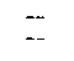 & $\overline{3.14}$ \\
\hline $6 \mathrm{~T}-2$ & 534911 & 120070 & High & $\begin{array}{l}\text { Terrace } \\
\text { slope }\end{array}$ & 2 & $\begin{array}{l}\text { Tal1-grass } \\
\text { prairie }\end{array}$ & Undisturbed & $\begin{array}{l}0-6 \\
6-12\end{array}$ & 52 & $\overline{30}$ & $\overline{18}$ & $\overline{\text { VGL }}$ & $\overline{23}$ & -- & $\overline{21}$ & $\overline{22}$ & $\overline{s c}$ & $\begin{array}{l}-- \\
-\end{array}$ & 3.19 \\
\hline $6 \mathrm{~T}-3$ & $5448 \mathrm{IV}$ & 849000 & H1gh & Upper flat & 2 & $\begin{array}{l}\text { Short-grass } \\
\text { prairie }\end{array}$ & $\begin{array}{l}\text { Rambutan } \\
\text { orchard }\end{array}$ & $\begin{array}{l}0-6 \\
6-12\end{array}$ & $\overline{33}$ & $\overline{48}$ & $\overrightarrow{19}$ & $\overline{\mathrm{L}}$ & $\overline{75}$ & $\overrightarrow{31}$ & $\overline{19}$ & $\overline{12}$ & $\overline{C L}$ & 0.78 & 2.69 \\
\hline $6 T-4$ & S349II & 120093 & Low & $\begin{array}{l}\text { Bottomland } \\
\text { depression }\end{array}$ & 0 & $\begin{array}{l}\text { Tal1-grass } \\
\text { prairie }\end{array}$ & Undisturbed & $\begin{array}{l}0-6 \\
6-12\end{array}$ & $\ddot{16}$ & $\overline{54}$ & $\overline{30}$ & $\overline{\mathrm{S} 1 \mathrm{CL}}$ & $\overline{87}$ & $\overline{30}$ & $\overline{--}$ & $\overline{12}$ & $\overline{\mathrm{CL}}$ & $\overline{0.70}$ & 2.61 \\
\hline $6 \mathrm{~T}-5$ & $5349 \mathrm{II}$ & 133032 & $\mathrm{H} 1 \mathrm{gh}$ & $\begin{array}{l}\text { Terrace } \\
\text { slope }\end{array}$ & 2 & $\begin{array}{l}\text { Ta11 scrub } \\
\text { woodland }\end{array}$ & Logged & $\begin{array}{l}0-6 \\
6-12\end{array}$ & $\overline{64}$ & $\overline{33}$ & $-\overline{3}$ & $\overline{\text { SL }}$ & $-\overline{49}$ & -- & 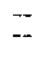 & $\overline{\mathrm{NP}}$ & $\overline{S M}$ & $0 . \overline{46}$ & $2 .-64$ \\
\hline $6 T-6$ & $5449 \mathrm{IV}$ & 969083 & Low & $\begin{array}{l}\text { Bottomland } \\
\text { flat }\end{array}$ & 0 & $\begin{array}{l}\text { Short-grass } \\
\text { prairie }\end{array}$ & $\begin{array}{l}\text { Cultivated } \\
\text { (1dle) }\end{array}$ & $\begin{array}{l}0-6 \\
6-12\end{array}$ & $\overline{58}$ & $\overline{26}$ & $\overline{16}$ & $\overline{\text { SL }}$ & -- & $\overline{25}$ & 15 & $\overline{10}$ & $\overline{\mathrm{sc}}$ & 0.55 & 2.65 \\
\hline $6 \mathrm{~T}-7$ & $5349 I I$ & 073103 & Low & $\begin{array}{l}\text { Bottonland } \\
\text { depression }\end{array}$ & 0 & $\begin{array}{l}\text { Short-grass } \\
\text { prairfe }\end{array}$ & Grazed & $\begin{array}{l}0-6 \\
6-12\end{array}$ & 10 & $\overline{64}$ & $\overline{26}$ & sil & $\overline{88}$ & -- & $\overline{20}$ & -- & $\overline{\mathrm{CL}}$ & $\overline{0.62}$ & 2.66 \\
\hline $6 \mathrm{~T}-8$ & $544 \mathrm{BIV}$ & 862788 & Low & $\begin{array}{l}\text { Bottomland } \\
\text { flat }\end{array}$ & 0 & $\begin{array}{l}\text { Short-grass } \\
\text { prairie }\end{array}$ & $\begin{array}{l}\text { Culctuated } \\
\text { (1dle) }\end{array}$ & $\begin{array}{l}0-6 \\
6-12\end{array}$ & -- & $\overline{21}$ & -2 & $\overline{\text { SL }}$ & $\overline{40}$ & $\overline{28}$ & $=$ & $\overline{\mathrm{NP}}$ & $\overline{s M}$ & 0.38 & $2 .-\overline{68}$ \\
\hline $6 \mathrm{~T}-9$ & 534921 & 207077 & Low & $\begin{array}{l}\text { Bottomland } \\
\text { flat }\end{array}$ & 0 & $\begin{array}{l}\text { Short-grass } \\
\text { pralrte }\end{array}$ & $\begin{array}{l}\text { Cultivated } \\
\text { (rice) }\end{array}$ & $\begin{array}{l}0-6 \\
6-12\end{array}$ & -- & $\overline{52}$ & $\overline{42}$ & sic & $\overline{95}$ & $\overline{47}$ & $\overline{30}$ & $\overline{17}$ & $\bar{M}$ & $\overline{1.05}$ & 2.71 \\
\hline $6 \mathrm{~T}-10$ & $5448 I v$ & 683910 & Low & $\begin{array}{l}\text { Bot tomland } \\
\text { flat }\end{array}$ & 0 & $\begin{array}{l}\text { Short-grass } \\
\text { prafrie }\end{array}$ & $\begin{array}{l}\text { Cultivated } \\
\text { (1dle) }\end{array}$ & $\begin{array}{l}0-6 \\
6-12\end{array}$ & $\overline{84}$ & $\overline{8}$ & $-\overline{8}$ & $\overrightarrow{\text { LS }}$ & $\overline{18}$ & $\overline{21}$ & $\ddot{14}$ & $-\overline{7}$ & $\overline{S M-S C}$ & -- & $-\overline{2.65}$ \\
\hline $6 \mathrm{~T}-11$ & $5348 I$ & 145970 & Low & $\begin{array}{l}\text { Bottomland } \\
\text { f1at }\end{array}$ & 0 & Woodland & $\begin{array}{l}\text { Coconut } \\
\text { orchard }\end{array}$ & $\begin{array}{l}0-6 \\
6-12\end{array}$ & 80 & -- & -- & $\overline{\text { LS }}$ & -- & -- & -- & $\overline{N P}$ & $\tilde{S M}$ & $\overline{0.70}$ & $\overline{2.65}$ \\
\hline $6 \mathrm{~T}-12$ & $5448 \mathrm{IV}$ & 915836 & Low & $\begin{array}{l}\text { Bottomland } \\
\text { flat }\end{array}$ & 0 & Savanna & $\begin{array}{l}\text { Rambutan } \\
\text { orchard }\end{array}$ & $\begin{array}{l}0-6 \\
6-12\end{array}$ & $\overline{86}$ & $\because 8$ & $--\overline{6}$ & $\overline{L S}$ & $\overrightarrow{17}$ & $\overline{17}$ & -- & $\overline{\mathrm{NP}}$ & $\mathrm{SH}$ & $=$ & 2.62 \\
\hline $6 \mathrm{~T}-13$ & S448IV & 873881 & Lov & $\begin{array}{l}\text { Bot tom land } \\
\text { flat }\end{array}$ & 0 & Savanna & $\begin{array}{l}\text { Cultivated } \\
\text { (sugar cane) }\end{array}$ & $\begin{array}{l}0-6 \\
6-12\end{array}$ & 79 & $\overline{17}$ & $\frac{-}{4}$ & $\overline{\text { LS }}$ & $\overline{27}$ & $=$ & -- & $\overline{\text { NP }}$ & $\overline{\text { SM }}$ & $-\therefore$ & $\overline{2.67}$ \\
\hline $6 T-14$ & $5448 \mathrm{III}$ & 893811 & Low & $\begin{array}{l}\text { Bottomland } \\
\text { flat }\end{array}$ & 0 & Low scrub & $\begin{array}{l}\text { Nipa palm } \\
\text { archard }\end{array}$ & $\begin{array}{l}0-6 \\
6-12\end{array}$ & $\overline{14}$ & $\overline{52}$ & $\overline{34}$ & $\overline{S i C L}$ & $\overline{91}$ & $\overline{77}$ & 33 & $\overline{44}$ & $\overline{\mathrm{CH}}$ & $\overline{4.15}$ & $2 .-60$ \\
\hline $6 T-15$ & S448III & 889791 & Low & $\begin{array}{l}\text { Bottomland } \\
\text { flat }\end{array}$ & 0 & Savanna & $\begin{array}{l}\text { Cultivated } \\
\text { (1die) }\end{array}$ & $\begin{array}{l}0-6 \\
6-12\end{array}$ & $\overline{18}$ & $\overline{44}$ & $\overline{38}$ & SicL & $\overline{85}$ & 60 & $\overline{36}$ & $\overline{24}$ & $\overline{M H}$ & 5.02 & $\overline{2.61}$ \\
\hline $6 \mathrm{~T}-16$ & $5448 \mathrm{IV}$ & 846936 & Los & $\begin{array}{l}\text { Bottomland } \\
\text { flat }\end{array}$ & 0 & Savanna & $\begin{array}{l}\text { Cultivated } \\
\text { (Idle) }\end{array}$ & $\begin{array}{l}0-6 \\
6-12\end{array}$ & $\overline{13}$ & s5 & $\overline{32}$ & $\overline{\mathrm{SICL}}$ & $\overline{93}$ & $\overline{32}$ & $\overline{21}$ & $\overline{11}$ & $\overline{\mathrm{CL}}$ & $-\overline{1.65}$ & $\overline{2 .-}$ \\
\hline $6 \mathrm{~T}-17$ & $5348 \mathrm{I}$ & 225970 & Low & $\begin{array}{l}\text { Bottomland } \\
\text { f lat }\end{array}$ & 0 & Low scrub & Logged & $\begin{array}{l}0-6 \\
6-12\end{array}$ & $\overline{19}$ & $\overline{58}$ & $\overline{23}$ & $\overrightarrow{\mathrm{S}} \overline{\mathrm{L}}$ & $\overline{8 B}$ & $\overline{37}$ & $\overline{20}$ & $\overline{17}$ & $\overline{C L}$ & $\overline{2.47}$ & 2.61 \\
\hline
\end{tabular}




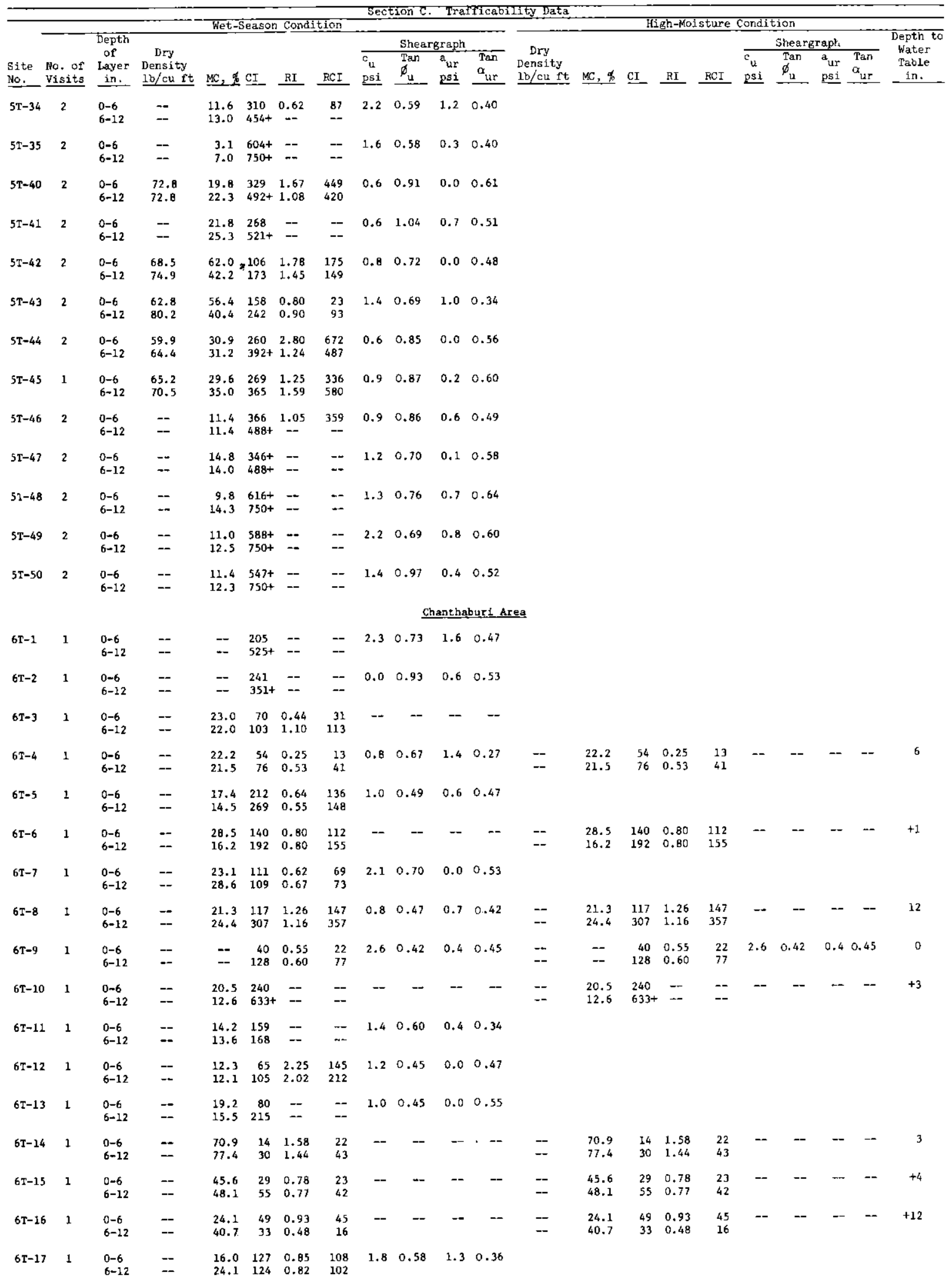




\begin{tabular}{|c|c|c|c|c|c|c|c|c|c|c|c|c|c|c|c|c|c|c|c|}
\hline \multirow{2}{*}{$\begin{array}{r}- \\
\text { Site } \\
\text { Sic. } \\
\text { ic. }\end{array}$} & \multirow{2}{*}{\multicolumn{2}{|c|}{ Location }} & \multirow{2}{*}{$\begin{array}{l}\text { Topog- } \\
\text { raphy }\end{array}$} & \multicolumn{3}{|c|}{ Da } & & \multirow{2}{*}{$\begin{array}{l}\text { Depth } \\
\text { of } \\
\text { Layer } \\
\text { in. }\end{array}$} & \multirow{2}{*}{\multicolumn{9}{|c|}{ Section B. Soil Data }} & \multirow{2}{*}{$\begin{array}{l}\text { or- } \\
\text { ganic } \\
\text { Con- } \\
\text { tent } \\
\text { ofio }\end{array}$} & \\
\hline & & & & & & & & & & & & & & & & & & & \\
\hline $6 \mathrm{~T}-18$ & $5448 \mathrm{IV}$ & 800847 & Low & id & 0 & Barren & Undisturbed & $0-6$ & -- & -- & -- & -- & -.. & -- & -. & -- & -- & - & -- \\
\hline & & & & f. & & & & $6-12$ & 56 & 31 & 13 & SL & 53 & 43 & 24 & 19 & CL & 2.87 & 2.65 \\
\hline $6 \mathrm{~T}-19$ & $5348 \mathrm{I}$ & 210950 & Low & Tidal flat & 0 & Low scrub & Logged & $0-6$ & -- &.- & -- & -- & -- & -- & -- & -- & -- & -- & -- \\
\hline & & & & & & & & & 40 & 53 & 7 & SiL & 73 & 71 & 49 & 22 & $\mathrm{MH}$ & -- & 2.64 \\
\hline $6 \mathrm{~T}-20$ & 53481 & 171936 & Low & Bot tomland & 0 & Shor & Cult & $0-6$ & -- & -- & -- & -- & -- & - & -- & -- & -- & -- & -- \\
\hline & & & & & & & & $6-12$ & 50 & 24 & 26 & SCL & 56 & 27 & 25 & 12 & $\mathrm{CL}$ & 0.86 & 2.63 \\
\hline $6 \mathrm{~T}-21$ & 53481 & 277879 & Low & Beach & 1 & Woodland & oconut & $0-6$ & -- & - & -- & -- & -- & -- & -- & -- & - & -- & -- \\
\hline & & & & & & & orc & 12 & 99 & 1 & 0 & s & 6 & -- & -- & NP & SP-SM & -- & 2.72 \\
\hline $6 \mathrm{~T}-22$ & $5448 \mathrm{III}$ & 901826 & Low & $\begin{array}{l}\text { Bottomland } \\
\text { flat }\end{array}$ & 0 & Short & Culti & $0-6$ & - & -- & -- & -- & -- & -- & -- & -- & - & -- & -- \\
\hline & & & & & & pralt & (id & $6-12$ & 30 & 47 & 23 & $\mathrm{~L}$ & 74 & 39 & 18 & 21 & CL & -- & 2.62 \\
\hline $6 \mathrm{~T}-23$ & 54481 II & 820809 & Low & $\begin{array}{l}\text { Bot tomland } \\
\text { f lat }\end{array}$ & 0 & Savanna & Cu1ti & $0-6$ & $\ddot{n}$ & -- & -- & $-\sim$ & -- & -- & -- & -- & - & $\ldots$ & -- \\
\hline & & & & & & & (id & -12 & 21 & 45 & 34 & $\mathrm{CL}$ & 84 & 75 & 38 & 37 & $\mathrm{MH}$ & 4.05 & 2.65 \\
\hline $6 \mathrm{~T}-24$ & $5348 \mathrm{I}$ & 195976 & Low & $\begin{array}{l}\text { Bottomland } \\
\text { flat }\end{array}$ & 0 & $\begin{array}{l}\text { Shart- } \\
\text { prairi }\end{array}$ & Culti & $0-6$ & -- & $\overline{7}$ & -- & -- & -- & -- & -- & -- & -- & -- & -- \\
\hline & & & & & & & & & 45 & 27 & 28 & $\mathrm{CL}$ & 60 & 34 & 19 & 15 & $\mathrm{CL}$ & 0.78 & 2.66 \\
\hline $6 \mathrm{~T}-25$ & S448IV & 810923 & Low & $\begin{array}{l}\text { Bottomland } \\
\text { flat }\end{array}$ & 0 & $\begin{array}{l}\text { Short- } \\
\text { prair }\end{array}$ & Culti & $0-6$ & -- & -- & -- & - & -- & -- & -- & -- & -- & -- & -- \\
\hline & & & & & & & (i & & 15 & 52 & 33 & SicL & 90 & 50 & 26 & 24 & $\mathrm{CH}$ & 0.70 & 2.66 \\
\hline $6 \mathrm{~T}-26$ & $5448 \mathrm{IV}$ & 976006 & H1gh & Upper slope & 4 & Savanna & bl & $0-6$ & -- & -- & - & -- & -- & -- & -- & -- & -- & -- & -- \\
\hline & & & & & & & plan & $6-12$ & 76 & 15 & 9 & GSL & 15 & 27 & 19 & B & sc & -- & 2.77 \\
\hline $6 \mathrm{~T}-27$ & 5349 II & 220081 & Low & $\begin{array}{l}\text { Bottomland } \\
\text { f lat }\end{array}$ & 0 & $\begin{array}{l}\text { Short- } \\
\text { prairie }\end{array}$ & ran & & -- & - & -- & -- & -- & -- & -- & -- & -- & - & -- \\
\hline & & & & 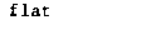 & & & & & 65 & 22 & 13 & SL & 40 & 22 & 14 & 8 & sc & 0.62 & 2.63 \\
\hline $6 \mathrm{~T}-28$ & $5448 \mathrm{IV}$ & 896980 & Low & $\begin{array}{l}\text { Bot tomland } \\
\text { flat }\end{array}$ & 1 & $\begin{array}{l}\text { Short- } \\
\text { prait }\end{array}$ & Cult1 & -6 & -- & -- & -- & -- & -- & - & -- & -- & -- & -- & -- \\
\hline & & & & flat & & & (1c & 12 & 14 & 59 & 27 & SiL & 90 & 49 & 34 & 15 & ML & 1.33 & 2.61 \\
\hline $6 \mathrm{~T}-29$ & $544 \mathrm{aIV}$ & 940017 & Low & $\begin{array}{l}\text { Bot tomland } \\
\text { flat }\end{array}$ & 0 & $\begin{array}{l}\text { Low ser } \\
\text { grass p }\end{array}$ & Cult & -6 & -- & & -- & -- & -- & -- & -- & -- & -- & -- & -- \\
\hline & & & & & & & (i & $6-12$ & 34 & 34 & 32 & CL & 71 & 58 & 32 & 26 & MH & 0.78 & 2.67 \\
\hline $6 \mathrm{~T}-30$ & 5449111 & 978060 & Low & $\begin{array}{l}\text { Bortomland } \\
\text { flat }\end{array}$ & 0 & $\begin{array}{l}\text { Low sc } \\
\text { grass }\end{array}$ & Cul & -6 & -- & -- & -- & -- & -- & - & -- & -- & -- & -- & -- \\
\hline & & & & & & & (1 & & 2 & 40 & 38 & CL & 75 & 53 & 33 & 20 & $\mathrm{MH}$ & 0.78 & 2.70 \\
\hline $6 \mathrm{~T}-31$ & $5449 \mathrm{III}$ & 993072 & Low & $\begin{array}{l}\text { Bottomland } \\
\text { flat }\end{array}$ & 0 & $\begin{array}{l}\text { Short } \\
\text { prair }\end{array}$ & Cult & $0-6$ & - & -- & -- & -- & $\cdots$ & -- & -- & -- & -- & -- & -- \\
\hline & & & & & & & & $6-12$ & 57 & 29 & 14 & SL & 52 & 27 & 15 & 12 & $\mathrm{CL}$ & 0.38 & 2.61 \\
\hline $6 \mathrm{~T}-32$ & 5449111 & I 924035 & Low & $\begin{array}{l}\text { tomland } \\
t\end{array}$ & 0 & Savanna & Rambutan & $0-6$ & -- & -- & -- & -- & -- & -- & -- & -- & -- & -- & -- \\
\hline & & & & & & & orchard & -12 & 22 & 47 & 31 & CL & 83 & 54 & 34 & 20 & МH & 1.33 & 2.65 \\
\hline $6 T-33$ & $544911 I$ & 932074 & Low & $\begin{array}{l}\text { mitand } \\
\text { ession }\end{array}$ & 0 & Woodland & ubt & & - & -- & - & -- & $\ldots$ & -- & - & -- & -- & -- & -- \\
\hline & & & & & & & Ia & $6-12$ & 50 & 30 & 20 & L & 55 & 32 & 20 & 12 & CL & 0.86 & 2.62 \\
\hline $6 T-34$ & $5348 \mathrm{I}$ & 217015 & Low & onland & 0 & $\begin{array}{l}\text { Short } \\
\text { prair }\end{array}$ & Cu1t & 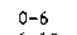 & -- & - & -- & -- & -- & -- & -- & -- & 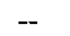 & -- & -- \\
\hline & & & & & & & $(r i$ & $6-12$ & 15 & 56 & 29 & Sict & 89 & 55 & 36 & 19 & $\mathrm{MH}$ & 2.35 & 2.63 \\
\hline $6 \mathrm{~T}-35$ & $5448 \mathrm{IV}$ & 775959 & Low & $\begin{array}{l}\text { Bottomland } \\
\text { f1at }\end{array}$ & 0 & Savann & Kamb & & $=$ & - & -- & -- & -- & - & -- & - & -- & -- & -- \\
\hline & & & & & & & orchara & $6-12$ & 59 & 29 & 12 & SL & 48 & 58 & 45 & 13 & SM & 2.87 & 2.81 \\
\hline $6 \tau-36$ & 534911 & 103019 & Low & $\begin{array}{l}\text { Bottom1and } \\
\text { flat }\end{array}$ & 0 & $\begin{array}{l}\text { Short-grass } \\
\text { praftre }\end{array}$ & & & -- & 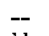 & - & -- & -- & -- & $\ldots$ & -- & $\ldots$ & -- & - \\
\hline & & & & Terrace flat & & & & & 87 & 11 & 2 & $\mathrm{~s}$ & 22 & -- & -- & NP & SM & 0.38 & 2.63 \\
\hline$T-37$ & $5449 \mathrm{III}$ & 784024 & Low & & 0 & Low & & & -- & - & - & -. & -- & - & -- & -- &.- & -. & -- \\
\hline & & & & & & & & $6-12$ & 65 & 20 & 15 & SL & 32 & 39 & 23 & 16 & sc & -- & 2.61 \\
\hline $6 \mathrm{~T}-38$ & $\$ 448 \mathrm{IV}$ & 825014 & Low & Upland flat & 0 & $\begin{array}{l}\text { Short-grass } \\
\text { prairie }\end{array}$ & & & -- & -- & -- & - & -- & -- & -- & -- & -- & -- & -- \\
\hline & & & & & & & ח & $6-12$ & 59 & 21 & 20 & SL & 40 & 42 & 26 & 16 & SM & 1.65 & 2.58 \\
\hline $6 \mathrm{~T}-39$ & $5448 \mathrm{IV}$ & 914864 & Low & Terrace flat & 0 & Savanna & & & - & -- & -- & -- & -- & -- & - & -- & -- & -- & -- \\
\hline & & & & & & & orchard & $6-12$ & 43 & 36 & 21 & I & 60 & 54 & 34 & 20 & MH & 1.55 & 2.62 \\
\hline $6 \mathrm{~T}-40$ & S449III & 821079 & $\mathrm{High}$ & Upland flat & 0 & Low scrub & & & -- & -- & -- & -- & -- & -- & -- & -- & - & -- & -- \\
\hline & & & & & & & (id & $6-12$ & 59 & 28 & 13 & SL & 50 & 41 & 29 & 12 & SM & 1.55 & 2.65 \\
\hline $6 T-41$ & $5449 I I I$ & 806024 & High & Upland $f$ lat & 0 & Savànna & & & -- & - & -- & -- & -- & -- & -- & -- & -- & $\ldots$ & .-. \\
\hline & & & & & & & & $6-12$ & 60 & 22 & 18 & si & 44 & 38 & 19 & 19 & sc & 0.70 & 2.62 \\
\hline $6 \mathrm{~T}-42$ & $5448 I I I$ & 009814 & Low & Terrace flat & 0 & Savanna & & & -- & -- & - & -- & -- & - & -- & -- & -- & -- & .- \\
\hline & & & & & & & pidate & $6-12$ & 63 & 24 & 13 & SL & 36 & 45 & 30 & 15 & SM & 1.33 & 2.61 \\
\hline $6 \mathrm{~T}-43$ & $5448 \mathrm{III}$ & 960793 & Low & land & 0 & Savanna & & & & - & -- & -- & -- & -- & -- & -- & -- & - & -- \\
\hline & & & & $\mathrm{f} 1$ & & & & & 77 & 14 & 9 & sL & 26 & - & -- & $\mathrm{NP}$ & SM & 1.45 & 2.60 \\
\hline $6 \mathrm{~T}-44$ & $5449 \mathrm{III}$ & 890050 & $\mathrm{H} 1 \mathrm{gh}$ & Upland flat & 0 & & Logged & & & & & & -- & -- & -- & -- & -- & -- & -- \\
\hline & & & & & & & & & 45 & 37 & 18 & L & 61 & 44 & 31 & 13 & ML. & 1.65 & 2.60 \\
\hline$T-455$ & $5448 \mathrm{IV}$ & 804004 & High & Upper slope & 15 & Savanna & & & & & - & & - & -- & - & -- & - & -- & -- \\
\hline & & & & & & & & & 39 & 17 & 24 & $c \mathbf{L}$ & 28 & 53 & 29 & 24 & SM & -- & 2.64 \\
\hline $6 T-465$ & $5448 \mathrm{IV}$ & 774983 & Low & land & 0 & Savanna & & & & & -- & -- & -- & -- & -- & - & & -- & -- \\
\hline & & & & & & & & & 49 & 37 & 14 & $\mathrm{~L}$ & 61 & 59 & 43 & 16 & мн & 1.98 & 2.73 \\
\hline $6 \mathrm{~T}-47$ & $5448 \mathrm{IV}$ & 790947 & Low & $\begin{array}{l}\text { Bot tomland } \\
\text { flat }\end{array}$ & 0 & $\begin{array}{l}\text { Short-grass } \\
\text { prafrie }\end{array}$ & & & & & -- & -- & - & 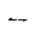 & -- & -. & & -- & -- \\
\hline & & & & & & & & & & 30 & 8 & SL & 55 & 57 & 45 & 12 & MH & 2.08 & 2.65 \\
\hline $1-4 B=3$ & $244817 \mathrm{~V}$ & 85 & Low & $\begin{array}{l}\text { Bottomland } \\
\text { flat }\end{array}$ & 0 & Savanna & plantation & $6-12$ & $\overline{31}$ & $\overline{55}$ & -- & $\overline{S i L}$ & -- & 39 & -- & - & -- & $\because$ & -- \\
\hline
\end{tabular}




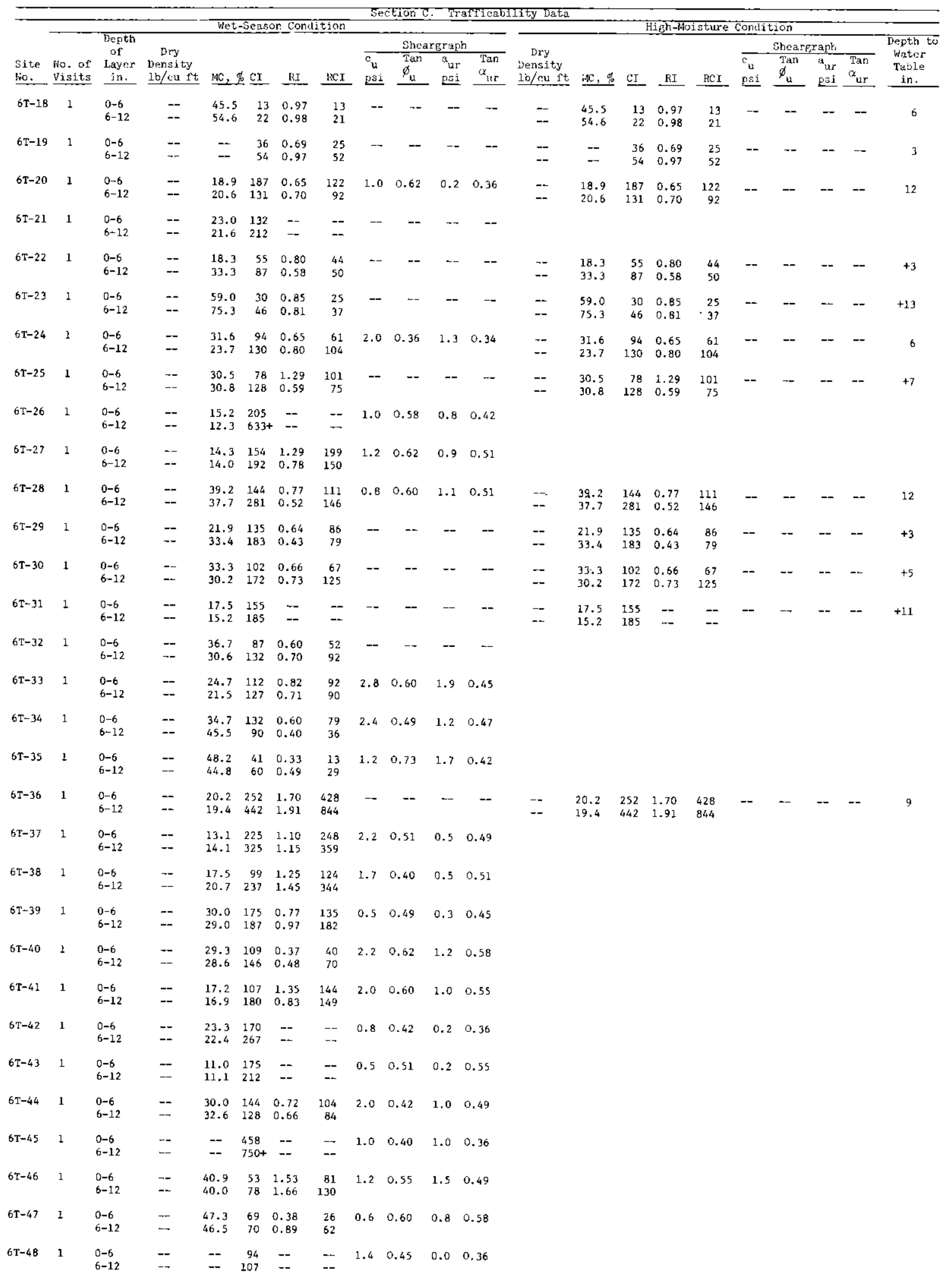


Table A3 (Continued)

\begin{tabular}{|c|c|c|c|c|c|c|c|c|c|c|c|c|c|c|c|c|c|c|c|}
\hline & & & 5 & Section A. S & Site Do & $\overline{a t a}$ & & & & & & cetion & B. S & 011 & Data & & & & \\
\hline & Locati & ion & & & & & & & & & & & & & USCS & & & or- & \\
\hline $\begin{array}{l}\text { Site } \\
\text { Iio. }\end{array}$ & $\begin{array}{l}\text { Map } \\
\text { Shcet }\end{array}$ & $\begin{array}{l}\text { Grid } \\
\text { Coor- } \\
\text { di- } \\
\text { nates }\end{array}$ & $\begin{array}{l}\text { Topols- } \\
\text { raphy } \\
\text { Clasis }\end{array}$ & $\begin{array}{l}\text { lopo- } \\
\text { graphic } \\
\text { Position }\end{array}$ & $\begin{array}{c}\text { slope } \\
\%\end{array}$ & Veqetation & Land Use & $\begin{array}{l}\text { Depth } \\
\text { of } \\
\text { Layer } \\
\text { in. }\end{array}$ & $\begin{array}{r}T e \\
\text { band } \\
\end{array}$ & $\begin{array}{r}\text { USI } \\
\text { exture } \\
\text { y wt, } \\
\text { Silt } \\
\end{array}$ & $\begin{array}{l}\text { e } \\
\frac{10}{\text { clay }}\end{array}$ & Type & $\begin{array}{l}\text { By wt } \\
\text { of } \\
\text { Fines }\end{array}$ & $\begin{array}{r}A t \\
\frac{L}{L}\end{array}$ & $\begin{array}{l}\text { atter- } \\
\text { berg } \\
\text { Linit } \\
\text { PL }\end{array}$ & $\frac{E S}{\text { PI }}$ & Type & $\begin{array}{l}\text { ganic } \\
\text { Con- } \\
\text { tent } \\
\% \\
\end{array}$ & $\begin{array}{l}\text { Spe- } \\
\text { cific } \\
\text { Grav- } \\
\text { ity }\end{array}$ \\
\hline $6 \mathrm{~T}-49$ & $5448 \mathrm{IV}$ & 995964 & Low & $\begin{array}{l}\text { Botromtand } \\
\text { flat }\end{array}$ & 0 & Savanna & $\begin{array}{l}\text { Rambutan } \\
\text { orchard }\end{array}$ & $\begin{array}{l}0-6 \\
6-12\end{array}$ & $\ddot{86}$ & $-\frac{-}{7}$ & $-\overline{7}$ & $\overline{L S}$ & $\overline{16}$ & $\overline{18}$ & -- & $\begin{array}{l}-- \\
\text { NP }\end{array}$ & $\overline{S M}$ & -- & 2.63 \\
\hline $6 \mathrm{~T}-50$ & $5448 \mathrm{IV}$ & 820970 & Low & $\begin{array}{l}\text { Bottomland } \\
\text { flat }\end{array}$ & 0 & Savanna & $\begin{array}{l}\text { Rubber } \\
\text { plantation }\end{array}$ & $\begin{array}{l}0-6 \\
6-12\end{array}$ & $\overline{60}$ & $\overline{21}$ & -- & $\overline{\text { SL }}$ & -- & 38 & -- & -- & $\overline{s c}$ & $\overline{0.95}$ & 2.61 \\
\hline $6 \mathrm{~T}-51$ & $5448 \mathrm{IV}$ & 877880 & Low & Lower slope & 25 & Savanna & $\begin{array}{l}\text { Rubber } \\
\text { plantation }\end{array}$ & $\begin{array}{l}0-6 \\
6-12\end{array}$ & $\overline{79}$ & $\overline{16}$ & $-\overline{5}$ & $\overline{\text { GLS }}$ & -- & $\overline{15}$ & $\begin{array}{l}-- \\
--\end{array}$ & $\overline{\mathrm{NP}}$ & $\overline{S M}$ & $\begin{array}{l}-- \\
--\end{array}$ & 2.57 \\
\hline $6 T-52$ & 5349 II & 145098 & Low & Terrace flat & $=0$ & $\begin{array}{l}\text { Short-grass } \\
\text { prairte }\end{array}$ & $\begin{array}{l}\text { Rubber } \\
\text { plantation }\end{array}$ & $\begin{array}{l}0-6 \\
6-12\end{array}$ & 57 & $\overline{29}$ & $\overline{14}$ & $\overline{\mathrm{SL}}$ & $\overline{53}$ & $\overline{25}$ & $\overline{20}$ & -- & $\overline{\mathrm{CL}-\mathrm{ML}}$ & $\overline{1.55}$ & $\overline{2.61}$ \\
\hline $6 \mathrm{~T}-53$ & $5448 \mathrm{IV}$ & 933858 & Low & Upland flat & 0 & Low scrub & $\begin{array}{l}\text { Rubber } \\
\text { plantation }\end{array}$ & $\begin{array}{l}0-6 \\
6-12\end{array}$ & -- & -- & -- & $\overline{\text { SL }}$ & 39 & -- & $\overline{30}$ & -- & $\overline{S M}$ & 2.65 & 2.59 \\
\hline $6 T-54$ & $5448 \mathrm{IV}$ & 790954 & High & Upper slope & 22 & $\begin{array}{l}\text { Tall scrub } \\
\text { savanna }\end{array}$ & $\begin{array}{l}\text { Rubber } \\
\text { plantation }\end{array}$ & $\begin{array}{l}0-6 \\
6-12\end{array}$ & $\ddot{49}$ & $\overline{40}$ & $\overline{11}$ & $\overline{\text { VGL }}$ & $\overline{26}$ & $\overline{56}$ & $\overline{37}$ & $\overline{19}$ & $\overline{G M}$ & -- & $3 .-\overline{30}$ \\
\hline $6 T-55$ & $5348 \mathrm{I}$ & 130952 & $\mathrm{High}$ & $\begin{array}{l}\text { Terrace } \\
\text { slope }\end{array}$ & 6 & $\begin{array}{l}\text { Low scrub; short- } \\
\text { grass prairie }\end{array}$ & Jndisturbed & $\begin{array}{l}0-6 \\
6-12\end{array}$ & so & $\overline{34}$ & $\overline{16}$ & $\overline{\text { GL }}$ & $\overline{41}$ & $\overline{29}$ & $\overline{22}$ & -- & $\overline{S M-S C}$ & $\begin{array}{l}-- \\
--\end{array}$ & 2.78 \\
\hline $6 T-56$ & 5448 III & 820834 & Low & $\begin{array}{l}\text { Bottoroland } \\
\text { flat }\end{array}$ & 0 & Savanna & Logged & $\begin{array}{l}0-6 \\
6-12\end{array}$ & $\overline{55}$ & $\overline{39}$ & -- & $\overline{\text { SL }}$ & -- & $\overline{76}$ & $\overline{44}$ & $\overline{32}$ & $\overline{\mathrm{MH}}$ & $\begin{array}{l}-- \\
--\end{array}$ & $-\overline{2.65}$ \\
\hline $6 \mathrm{~T}-57$ & $5448 \mathrm{III}$ & 817816 & Low & $\begin{array}{l}\text { Bot tomland } \\
\text { flat }\end{array}$ & 0 & $\begin{array}{l}\text { Short-grass } \\
\text { prairie }\end{array}$ & Grazed & $\begin{array}{l}0-6 \\
6-12\end{array}$ & $\overline{94}$ & $\frac{-1}{4}$ & $-\overline{2}$ & $\bar{s}$ & $-\overline{9}$ & $\begin{array}{l}-- \\
--\end{array}$ & $\overline{--}$ & $\overline{\mathrm{NP}}$ & $S P-S M$ & 2.47 & 2.63 \\
\hline $6 T-58$ & S349II & 150065 & Low & $\begin{array}{l}\text { Bot tomland } \\
\text { flat }\end{array}$ & 0 & $\begin{array}{l}\text { Tal1 scrub } \\
\text { woodland }\end{array}$ & Undisturbed & $\begin{array}{l}0-6 \\
6-12\end{array}$ & $\overline{55}$ & $\overline{28}$ & $\overline{17}$ & $\overline{\text { SL }}$ & $\overline{s 1}$ & -- & 15 & -- & $\overline{\mathrm{CL}-\mathrm{HL}}$ & $\overline{0.95}$ & $-\overline{2.63}$ \\
\hline $6 \mathrm{~T}-59$ & $53491 \mathrm{I}$ & 159089 & Low & $\begin{array}{l}\text { Battomland } \\
\text { flat }\end{array}$ & 0 & $\begin{array}{l}\text { Low scrub; short- } \\
\text { grass prairle }\end{array}$ & $\begin{array}{l}\text { Rubber } \\
\text { plantation }\end{array}$ & $\begin{array}{l}0-6 \\
6-12\end{array}$ & $\overline{20}$ & $\overline{38}$ & $\overline{42}$ & $\bar{c}$ & $\overline{-1}$ & $\overline{52}$ & $\overline{27}$ & $\overline{25}$ & $\overline{\mathrm{CH}}$ & $\overline{0.95}$ & $\begin{array}{l}-- \\
2.62\end{array}$ \\
\hline $6 T-60$ & $5349 I I$ & 187064 & High & Upland f lat & 0 & $\begin{array}{l}\text { Tall scrub } \\
\text { forest }\end{array}$ & Undisturbed & $\begin{array}{l}0-6 \\
6-12\end{array}$ & $\overline{51}$ & $\overline{35}$ & $\overline{14}$ & $\overline{\mathrm{L}}$ & $\overline{59}$ & $\overline{18}$ & -- & $\overrightarrow{\mathrm{NP}}$ & $\overline{\mathrm{ML}}$ & $\overline{0.55}$ & $\overline{2.64}$ \\
\hline $6 \mathrm{~T}-61$ & $5348 \mathrm{I}$ & 152984 & High & Upland flat & 0 & Low scrub & Undisturbed & $\begin{array}{l}0-6 \\
6-12\end{array}$ & $-\overline{60}$ & $\overline{28}$ & $\overline{12}$ & $\overline{G S L}$ & $\overline{29}$ & $\overline{24}$ & $\overline{16}$ & -- & $\overline{\mathrm{sC}}$ & $\overline{0.95}$ & $\overline{2.99}$ \\
\hline $6 \mathrm{~T}-62$ & $5448 \mathrm{IV}$ & 767963 & Low & $\begin{array}{l}\text { Bot tomland } \\
\text { depression }\end{array}$ & 0 & Sa:anna & $\begin{array}{l}\text { Rubber } \\
\text { plantation }\end{array}$ & $\begin{array}{l}0-6 \\
6-12\end{array}$ & $\overline{70}$ & $\overline{21}$ & $-\overline{9}$ & $\overline{\mathrm{GSL}}$ & $\overline{19}$ & $\overline{36}$ & $\overline{24}$ & $\overline{12}$ & $\overline{\mathrm{sc}}$ & -- & $\begin{array}{l}-- \\
2.85\end{array}$ \\
\hline
\end{tabular}




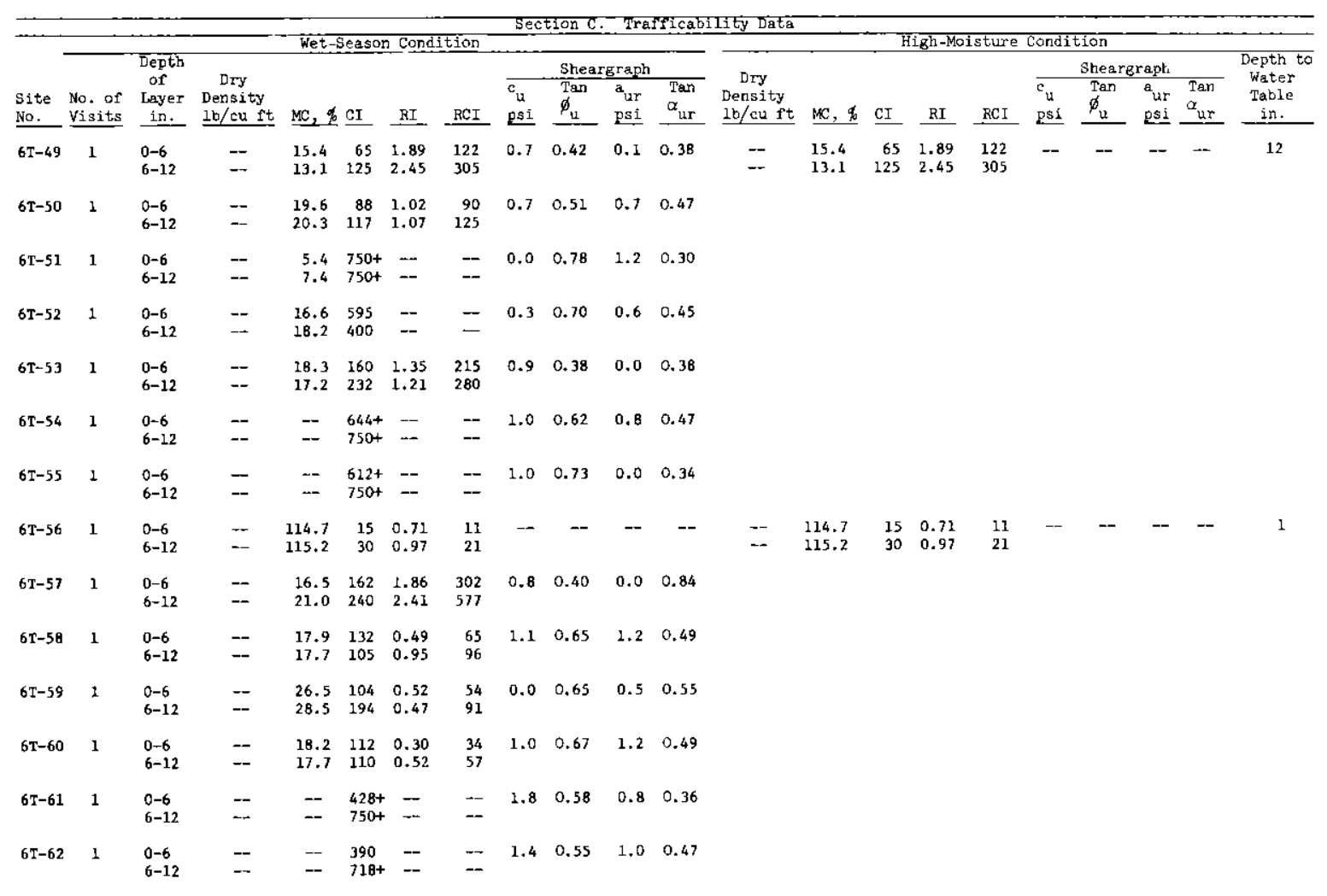


Table A4

Soil Mo1sture-Strength Study

Summary of Site, Soil, and Trafficability Data

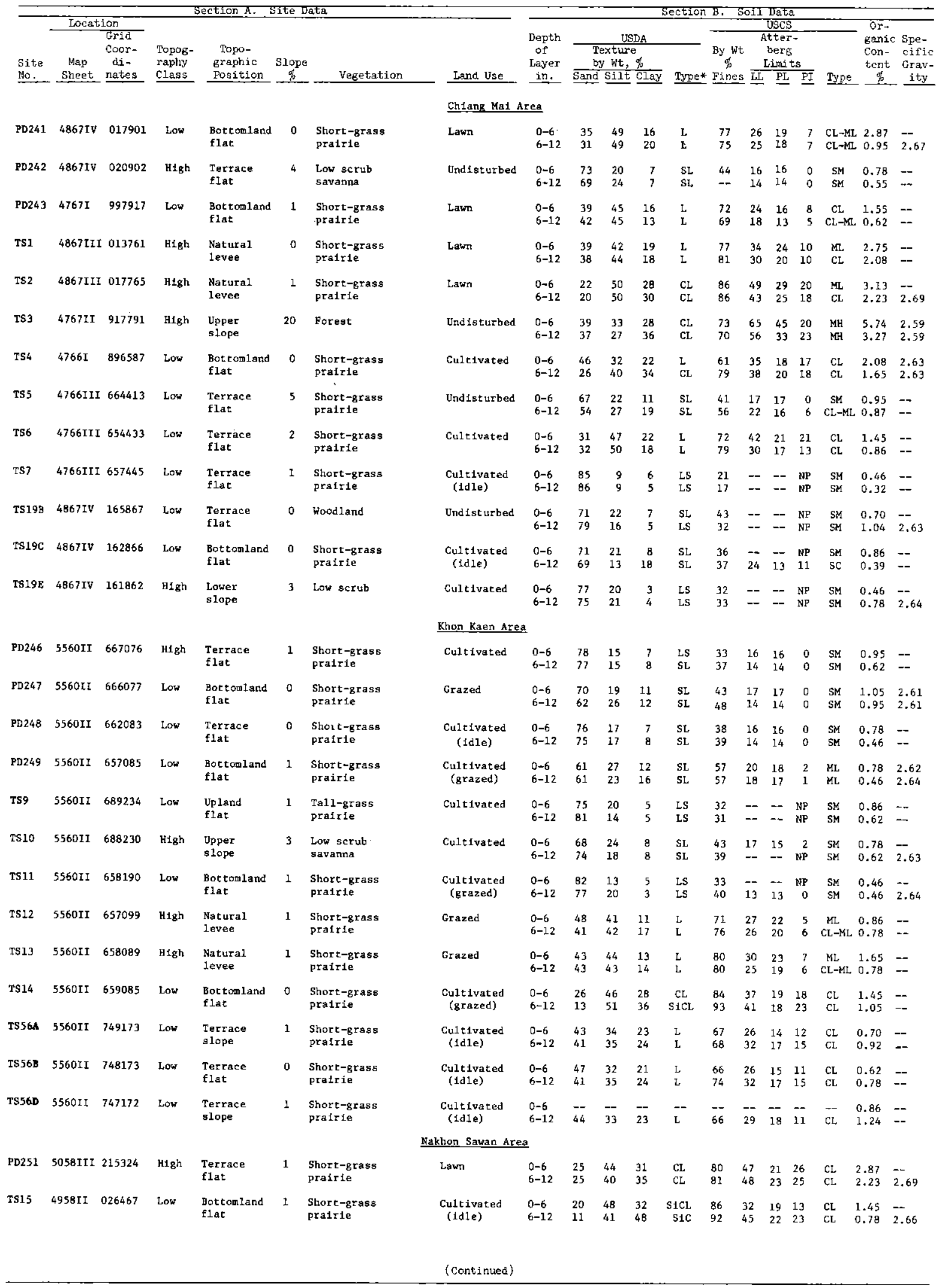




\begin{tabular}{|c|c|c|c|c|c|c|c|c|c|c|c|c|c|c|c|c|c|c|c|c|}
\hline \multirow[b]{3}{*}{$\begin{array}{l}\text { Site } \\
\text { No.-. }\end{array}$} & \multicolumn{11}{|c|}{ Wet-Season Condition } & \multicolumn{9}{|c|}{ IMgh-Moisture Condition } \\
\hline & \multirow{3}{*}{$\begin{array}{l}\text { No. of } \\
\text { Visits } \\
\end{array}$} & \multirow{3}{*}{$\begin{array}{l}\text { Depth } \\
\text { of } \\
\text { Layer } \\
\text { in. } \\
\end{array}$} & \multirow{3}{*}{$\begin{array}{c}\text { Dry } \\
\text { Density } \\
\text { lb/cu ft }\end{array}$} & \multirow{3}{*}{$\underline{M C, q}$} & \multirow{3}{*}{$\underline{\mathrm{CI}}$} & \multirow{3}{*}{$\underline{\mathrm{RI}}$} & \multirow{3}{*}{$\underline{\mathrm{RCI}}$} & \multicolumn{4}{|c|}{ Shesrgraph $* *$} & \multirow{3}{*}{$\begin{array}{l}\text { Dry } \\
\text { Density } \\
\text { lo/cu st } \\
\end{array}$} & \multirow{3}{*}{$\underline{M C, q}$} & \multirow{3}{*}{$\underline{\mathrm{CI}}$} & \multirow{3}{*}{$\underline{\mathrm{RI}}$} & \multirow{3}{*}{$\underline{\mathrm{RCI}}$} & \multicolumn{3}{|c|}{ Sheargragli** } & \multirow{2}{*}{$\begin{array}{l}\text { Depth to } \\
\text { Water } \\
\text { Tablet } \\
\text { in. }\end{array}$} \\
\hline & & & & & & & & $\begin{array}{l}c_{u} \\
\text { psi }\end{array}$ & $\stackrel{p}{p e n}_{u}$ & $\begin{array}{l}\text { a }{ }^{\text {ur }} \\
\text { pgi }\end{array}$ & $\begin{array}{l}\text { Tan } \\
\alpha_{u x} \\
\end{array}$ & & & & & & $\begin{array}{l}c_{u} \\
\underline{p s i}\end{array}$ & $\begin{array}{l}\text { Tenn } \\
\emptyset_{\mathrm{u}} \\
\end{array}$ & 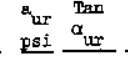 & \\
\hline \multicolumn{9}{|c|}{ Chiang Ma1 Area } & & & & & & & & & & & & \\
\hline PD241 & 19 & $\begin{array}{l}0-6 \\
6-12\end{array}$ & $\begin{array}{l}94.8 \\
97.6\end{array}$ & $\begin{array}{l}25.9 \\
20.5\end{array}$ & $\begin{array}{l}100+ \\
212+\end{array}$ & $\begin{array}{l}+--. \\
+0.68\end{array}$ & $\ddot{150+}$ & -- & -- & -- & -- & $\overline{--}$ & $\begin{array}{l}28.2 \\
19.4\end{array}$ & $\begin{array}{l}35 \\
96\end{array}$ & $\overline{0.29}$ & $\overline{28}$ & & & & 1 \\
\hline $\mathrm{PD} 242$ & 18 & $\begin{array}{l}0-6 \\
6-12\end{array}$ & $\begin{array}{l}93.4 \\
97.8\end{array}$ & $\begin{array}{l}12.8 \\
11.7\end{array}$ & $\begin{array}{l}156 \\
177\end{array}$ & 2.60 & $\overline{472}$ & 3.2 & 0.58 & 0.7 & 0.43 & & & & & & & & & \\
\hline PD243 & 18 & $\begin{array}{l}0-6 \\
6-12\end{array}$ & $\begin{array}{r}97.9 \\
109.8\end{array}$ & $\begin{array}{l}20.4 \\
15.0\end{array}$ & $\begin{array}{l}192+ \\
348+\end{array}$ & +--- & $\overline{184+}$ & 3.8 & 0.58 & 0.9 & 0.70 & $\begin{array}{r}98.7 \\
113.8\end{array}$ & $\begin{array}{l}19.2 \\
15.3\end{array}$ & $\begin{array}{r}90 \\
121\end{array}$ & $\tilde{0.44}$ & 53 & & & & 23 \\
\hline TS1 & 14 & $\begin{array}{l}0-6 \\
6-12\end{array}$ & $\begin{array}{l}86.2 \\
89.3\end{array}$ & $\begin{array}{l}19.4 \\
15.6\end{array}$ & $\begin{array}{l}497+ \\
661+\end{array}$ & $+\overline{1.01}$ & $491+$ & -- & -- & -- & -- & & & & & & & & & \\
\hline TS2 & 16 & $\begin{array}{l}0-6 \\
6-12\end{array}$ & $\begin{array}{l}79.5 \\
84.9\end{array}$ & $\begin{array}{l}28.8 \\
26.2\end{array}$ & $\begin{array}{l}191 \\
265+\end{array}$ & $+\overline{1.02}$ & $\overline{247}$ & 3.1 & 0.68 & 0.2 & 0.63 & & & & & & & & & \\
\hline TS3 & 10 & $\begin{array}{l}0-6 \\
6-12\end{array}$ & $\begin{array}{l}67.8 \\
63.5\end{array}$ & $\begin{array}{l}42.3 \\
33.7\end{array}$ & $\begin{array}{r}60 \\
106\end{array}$ & 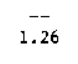 & $\overline{141}$ & 2.6 & 0.68 & 0.7 & 0.70 & & & & & & & & & \\
\hline TS4 & 14 & $\begin{array}{l}0-6 \\
6-12\end{array}$ & $\begin{array}{l}89.8 \\
98.0\end{array}$ & $\begin{array}{l}29.0 \\
25.2\end{array}$ & $\begin{array}{r}68 \\
126\end{array}$ & $\overline{0.86}$ & $\overline{109}$ & 3.3 & 0.19 & 1.6 & 0.19 & $\overline{-}$ & $\begin{array}{l}29.6 \\
25.1\end{array}$ & $\begin{array}{r}51 \\
102\end{array}$ & $\overline{0.69}$ & -- & & & & 0 \\
\hline TS5 & 15 & $\begin{array}{l}0-6 \\
6-12\end{array}$ & $\begin{array}{l}91.1 \\
89.3\end{array}$ & $\begin{array}{l}11.8 \\
11.5\end{array}$ & $\begin{array}{l}297+ \\
369+\end{array}$ & $\begin{array}{l}+- \\
+1.64 \\
+1.64\end{array}$ & $\overline{421+}$ & 1.5 & 0.78 & 1.2 & 0.75 & & & & & & & & & \\
\hline TS6 & 19 & $\begin{array}{l}0-6 \\
6-12\end{array}$ & $\begin{array}{r}91.7 \\
104.6\end{array}$ & $\begin{array}{l}28.9 \\
21.2\end{array}$ & $\begin{array}{c}78 \\
137+\end{array}$ & $\begin{array}{c}-- \\
+0.78\end{array}$ & $\overline{103+}$ & -- & -- & - & -- & -- & $\begin{array}{l}34.5 \\
23.6\end{array}$ & $\begin{array}{r}60 \\
129\end{array}$ & 0.35 & $-\overline{45}$ & & & & 0 \\
\hline TS7 & 8 & $\begin{array}{l}0-6 \\
6-12\end{array}$ & $\begin{array}{l}88.3 \\
86.6\end{array}$ & $\begin{array}{l}28.6 \\
28.8\end{array}$ & $\begin{array}{l}173 \\
279\end{array}$ & $\overline{0.99}$ & $\overline{276}$ & -- & -- & -- & -- & -- & $\begin{array}{l}29.3 \\
29.9\end{array}$ & $\begin{array}{l}136 \\
224\end{array}$ & 0.77 & $\overline{172}$ & & & & 0 \\
\hline TS19B & 8 & $\begin{array}{l}0-6 \\
6-12\end{array}$ & $\begin{array}{l}93.2 \\
95.4\end{array}$ & $\begin{array}{l}17.2 \\
14.3\end{array}$ & $\begin{array}{l}263 \\
297\end{array}$ & -- & $\overline{-}$ & -- & -- & -- & -- & & & & & & & & & \\
\hline $\mathrm{TS} 19 \mathrm{C}$ & 9 & $\begin{array}{l}0-6 \\
6-12\end{array}$ & $\begin{array}{r}96.0 \\
101.6\end{array}$ & $\begin{array}{l}21.9 \\
17.5\end{array}$ & $\begin{array}{l}170 \\
167\end{array}$ & $\overline{0.37}$ & $\overline{62}$ & -- & -- & -- & -- & -- & $\begin{array}{l}21.6 \\
20.1\end{array}$ & $\begin{array}{l}169 \\
147\end{array}$ & $\overline{0.17}$ & $\overline{25}$ & & & & 1 \\
\hline TS198 & 9 & $\begin{array}{l}0-6 \\
6-12\end{array}$ & $\begin{array}{l}90.2 \\
94.0\end{array}$ & $\begin{array}{l}11.2 \\
10.2\end{array}$ & $\begin{array}{l}142 \\
239\end{array}$ & $\overline{2.14}$ & 474 & -- & -- & -- & -- & & & & & & & & & \\
\hline \multicolumn{21}{|c|}{ Xhon Kaen Area } \\
\hline PD246 & 7 & $\begin{array}{l}0-6 \\
6-12\end{array}$ & $\begin{array}{r}96.4 \\
100.1\end{array}$ & $\begin{array}{l}12.1 \\
11.7\end{array}$ & $\begin{array}{l}181+ \\
230+\end{array}$ & $\begin{array}{l}+-- \\
+2.15\end{array}$ & $\overline{442+}$ & 0.6 & 0.76 & 0.3 & 0.60 & & & & & & & & & \\
\hline PD247 & 6 & $\begin{array}{l}0-6 \\
6-12\end{array}$ & $\begin{array}{l}101.1 \\
105.2\end{array}$ & $\begin{array}{l}17.3 \\
16.5\end{array}$ & $\begin{array}{l}151 \\
200+\end{array}$ & $\begin{array}{c}-- \\
+0.55\end{array}$ & $\overline{117+}$ & -- & -- & - & - & $\overline{-}$ & $\begin{array}{l}16.2 \\
17.1\end{array}$ & $\begin{array}{c}92 \\
153+\end{array}$ & $0 .--$ & $\overline{54+}$ & & & & 1 \\
\hline PD248 & 7 & $\begin{array}{l}0-6 \\
6-12\end{array}$ & $\begin{array}{r}99.7 \\
104.6\end{array}$ & $\begin{array}{l}18.9 \\
15.6\end{array}$ & $\begin{array}{l}184+ \\
344+\end{array}$ & $\begin{array}{l}+-- \\
+0.86\end{array}$ & $\begin{array}{l}-- \\
332+\end{array}$ & 0.8 & 0.68 & 0.8 & 0.47 & $\overline{--}$ & $\begin{array}{l}19.1 \\
15.1\end{array}$ & $\begin{array}{l}188+ \\
406+\end{array}$ & 0.29 & $\overline{118+}$ & & & & 0 \\
\hline PD 249 & 7 & $\begin{array}{l}0-6 \\
6-12\end{array}$ & $\begin{array}{l}95.8 \\
97.4\end{array}$ & $\begin{array}{l}17.5 \\
15.1\end{array}$ & $\begin{array}{l}211+ \\
504+\end{array}$ & $+\overline{+}+\overline{09}$ & $\overline{547+}$ & 0.6 & 0.84 & 0.9 & 0.32 & -- & $\begin{array}{l}18.8 \\
16.6\end{array}$ & $\begin{array}{l}185 \\
388+\end{array}$ & 0.80 & $310+$ & & & & 18 \\
\hline TS9 & 6 & $\begin{array}{l}0-6 \\
6-12\end{array}$ & $\begin{array}{l}97.5 \\
99.1\end{array}$ & $\begin{array}{l}12.4 \\
12.2\end{array}$ & $\begin{array}{c}87 \\
160+\end{array}$ & $\begin{array}{r}-\overline{-} \\
+1.59\end{array}$ & $-\overline{242+}$ & 0.8 & 0.58 & 0.4 & 0.60 & & & & & & & & & \\
\hline TS10 & 4 & $\begin{array}{l}0-6 \\
6-12\end{array}$ & $\begin{array}{l}89.5 \\
93.2\end{array}$ & $\begin{array}{l}13.9 \\
13.4\end{array}$ & $\begin{array}{r}95 \\
101\end{array}$ & $\overline{1.87}$ & $\overline{193}$ & 1.1 & 0.54 & 0.6 & 0.47 & & & & & & & & & \\
\hline TS11 & 4 & $\begin{array}{l}0-6 \\
6-12\end{array}$ & $\begin{array}{l}102.6 \\
103.5\end{array}$ & $\begin{array}{l}16.2 \\
16.1\end{array}$ & $\begin{array}{l}180 \\
240\end{array}$ & $\overline{0.78}$ & 190 & 0.7 & 0.49 & 0.6 & 0.47 & -- & $\begin{array}{l}17.0 \\
16.8\end{array}$ & $\begin{array}{l}187 \\
246\end{array}$ & $\overline{0.42}$ & 103 & 0.7 & 0.49 & 0.60 .47 & 4 \\
\hline TS12 & 6 & $\begin{array}{l}0-6 \\
6-12\end{array}$ & $\begin{array}{l}96.8 \\
97.2\end{array}$ & $\begin{array}{l}19.3 \\
20.4\end{array}$ & $\begin{array}{l}182 \\
178\end{array}$ & $\overline{0.62}$ & $\overline{102}$ & 2.2 & 0.44 & 0.2 & 0.50 & & & & & & & & & \\
\hline TS13 & 7 & $\begin{array}{l}0-6 \\
6-12\end{array}$ & $\begin{array}{l}91.1 \\
92.4\end{array}$ & $\begin{array}{l}19.5 \\
20.1\end{array}$ & $\begin{array}{l}191+ \\
157\end{array}$ & $+\overline{0.69}$ & $1-\overline{115}$ & 2.2 & 0.64 & 0.5 & 0.54 & & & & & & & & & \\
\hline TS14 & 6 & $\begin{array}{l}0-6 \\
6-12\end{array}$ & $\begin{array}{l}92.8 \\
91.0\end{array}$ & $\begin{array}{l}22.3 \\
26.2\end{array}$ & $\begin{array}{l}215+ \\
206+\end{array}$ & +--- & $209+$ & 0.0 & 1.15 & 0.0 & 0.58 & $\overline{--}$ & $\begin{array}{l}27.2 \\
28.1\end{array}$ & $\begin{array}{r}68 \\
115\end{array}$ & $0 . \overline{0.76}$ & $\overline{87}$ & 0.0 & 1.15 & 0.00 .58 & 3 \\
\hline TS56A & 4 & $\begin{array}{l}0-6 \\
6-12\end{array}$ & $\begin{array}{l}91.9 \\
95.4\end{array}$ & $\begin{array}{l}19.7 \\
20.0\end{array}$ & $\begin{array}{l}118 \\
214+\end{array}$ & +0.90 & $\overline{197+}$ & -- & -- & -- & -- & $\begin{array}{l}95.4 \\
97.6\end{array}$ & $\begin{array}{l}19.7 \\
20.4\end{array}$ & $\begin{array}{r}94 \\
172\end{array}$ & 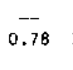 & $\overline{134}$ & & & & 6 \\
\hline TS56B & 3 & $\begin{array}{l}0-6 \\
6-12\end{array}$ & $\begin{array}{l}98.0 \\
99.3\end{array}$ & $\begin{array}{l}21.3 \\
21.9\end{array}$ & $\begin{array}{r}86 \\
117\end{array}$ & $\overline{0.78}$ & $\overline{91}$ & -- & -- & - & - & $\overline{--}$ & $\begin{array}{l}21.6 \\
22.0\end{array}$ & $\begin{array}{r}82 \\
101\end{array}$ & 0.76 & $\overline{77}$ & & & & 0 \\
\hline TS56D & 3 & $\begin{array}{l}0-6 \\
6-12\end{array}$ & $\begin{array}{l}99.5 \\
93.0\end{array}$ & $\begin{array}{l}17.4 \\
21.1\end{array}$ & $\begin{array}{l}131 \\
123\end{array}$ & $\overline{0.74}$ & $\overline{91}$ & -- & -- & -- & -- & $\begin{array}{l}98.2 \\
92.3\end{array}$ & $\begin{array}{l}17.3 \\
20.7\end{array}$ & $\begin{array}{l}142 \\
116\end{array}$ & 0.73 & $-\overline{85}$ & & & & 20 \\
\hline \multicolumn{21}{|c|}{ Nakhon Sawan Area } \\
\hline PD251 & 8 & $\begin{array}{l}0-6 \\
6-12\end{array}$ & $\begin{array}{l}81.2 \\
89.3\end{array}$ & $\begin{array}{l}21.8 \\
21.7\end{array}$ & $\begin{array}{l}232+ \\
362+\end{array}$ & $-\overline{1.12}$ & $\overline{366+}$ & 2,2 & 0.52 & 3.0 & 0.55 & & & & & & & & & \\
\hline TS15 & 9 & $\begin{array}{l}0-6 \\
6-12\end{array}$ & $\begin{array}{l}74.9 \\
83.0\end{array}$ & $\begin{array}{l}26.2 \\
25.2\end{array}$ & $\begin{array}{l}112+ \\
229+\end{array}$ & $-\overline{1.09}$ & $\overline{246+}$ & 3.7 & 0.43 & 1.5 & 0.65 & -- & $\begin{array}{l}25.9 \\
25.4\end{array}$ & $\begin{array}{r}66 \\
122\end{array}$ & $\overline{0.88}$ & $\overline{107}$ & & & & 0 \\
\hline & & & & & & & & & & (Conti & (nued) & & & & & & & & & \\
\hline
\end{tabular}

\footnotetext{
* $c_{u}$, ultimate soil-to-soil cohesion; $\phi_{u}$, ultimate soil-to-soil angle of internal friction; $a_{u r}$, wltimate soil-to-rubber adhesion; $\alpha_{u r}$, wltimate soil-to-rubber angle of friction.

+ Plus (+) denotes depth of water above surface. 


\begin{tabular}{|c|c|c|c|c|c|c|c|c|c|c|c|c|c|c|c|c|c|c|c|}
\hline \multirow{3}{*}{\begin{tabular}{l} 
\\
\cline { 2 - 2 } \\
Sitc \\
iio.
\end{tabular}} & \multirow{2}{*}{\multicolumn{2}{|c|}{ Iocation }} & \multirow{3}{*}{$\begin{array}{l}\text { Se } \\
\text { Topog- } \\
\text { raphy } \\
\text { Class } \\
\end{array}$} & \multirow{3}{*}{$\begin{array}{l}\text { Section A. S } \\
\text { Topo- } \\
\text { graphic } \\
\text { Position }\end{array}$} & \multirow{3}{*}{$\begin{array}{l}\text { Site Da } \\
\text { Slope } \\
\text { of }\end{array}$} & \multirow[b]{3}{*}{ Vogetation } & \multirow[b]{3}{*}{ Land Use } & \multirow[b]{3}{*}{$\begin{array}{l}\text { Depth } \\
\text { of } \\
\text { Layer } \\
\text { in. } \\
\end{array}$} & \multirow{2}{*}{\multicolumn{9}{|c|}{$\begin{array}{r}\text { Section B. Soil Data } \\
\text { USCS }\end{array}$}} & \multirow[b]{3}{*}{$\begin{array}{c}\text { Or- } \\
\text { ganic } \\
\text { Con- } \\
\text { tent } \\
\% \\
\%\end{array}$} & \multirow[b]{3}{*}{$\begin{array}{l}\text { Spe- } \\
\text { cific } \\
\text { Grav- } \\
\text { ity }\end{array}$} \\
\hline & & & & & & & & & & & & & & & & & & & \\
\hline & $\begin{array}{c}\text { Yap } \\
\text { Slicet }\end{array}$ & $\begin{array}{c}\text { Grid } \\
\text { Coor- } \\
\text { di- } \\
\text { nates } \\
\end{array}$ & & & & & & & \multicolumn{3}{|c|}{$\begin{array}{r}\text { USDA } \\
\text { Texture } \\
\text { by Wt, of } \\
\end{array}$} & Type & \multicolumn{4}{|c|}{$\begin{array}{cc}\text { Btter- } \\
\text { By wt } \\
\text { berg } \\
\text { Limits }\end{array}$} & Type & & \\
\hline TS16 & $5058 I I I$ & 171364 & Low & $\begin{array}{l}\text { Bottomland } \\
\text { depression }\end{array}$ & 1 & $\begin{array}{l}\text { Low scrub } \\
\text { savanna }\end{array}$ & Cultivated & $\begin{array}{l}0-6 \\
6-12\end{array}$ & $\begin{array}{r}10 \\
5\end{array}$ & $\begin{array}{l}34 \\
33\end{array}$ & $\begin{array}{l}56 \\
62\end{array}$ & $\begin{array}{l}\mathrm{c} \\
\mathrm{c}\end{array}$ & $\begin{array}{l}96 \\
98\end{array}$ & $\begin{array}{l}64 \\
81\end{array}$ & $\begin{array}{l}32 \\
35\end{array}$ & $\begin{array}{l}32 \\
46\end{array}$ & $\begin{array}{l}\mathrm{MH} \\
\mathrm{CH}\end{array}$ & $\begin{array}{l}2.35 \\
1.88\end{array}$ & $\overline{2.70}$ \\
\hline TS17 & $5057 \mathrm{IV}$ & 206249 & Low & $\begin{array}{l}\text { Bottomland } \\
\text { flat }\end{array}$ & 1 & $\begin{array}{l}\text { Short-grass } \\
\text { prairie }\end{array}$ & Culcivated & $\begin{array}{l}0-6 \\
6-12\end{array}$ & $\begin{array}{l}44 \\
41\end{array}$ & $\begin{array}{l}34 \\
33\end{array}$ & $\begin{array}{l}22 \\
26\end{array}$ & $\begin{array}{l}\text { L } \\
\text { L }\end{array}$ & $\begin{array}{l}67 \\
68\end{array}$ & $\begin{array}{l}40 \\
43\end{array}$ & $\begin{array}{l}20 \\
24\end{array}$ & $\begin{array}{l}20 \\
19\end{array}$ & $\begin{array}{l}\mathrm{CL} \\
\mathrm{CL}\end{array}$ & $\begin{array}{l}2.87 \\
2.35\end{array}$ & $\begin{array}{l}2.67 \\
--\end{array}$ \\
\hline TS18 & $5057 \mathrm{IV}$ & 207239 & Low & $\begin{array}{l}\text { Bottomland } \\
\text { flat }\end{array}$ & 0 & $\begin{array}{l}\text { Short-grass } \\
\text { pralrie }\end{array}$ & Cultivated & $\begin{array}{l}0-6 \\
6-12\end{array}$ & $\begin{array}{l}62 \\
59\end{array}$ & $\begin{array}{l}29 \\
27\end{array}$ & $\begin{array}{r}9 \\
14\end{array}$ & $\begin{array}{l}\text { SL } \\
\text { SL }\end{array}$ & $\begin{array}{l}54 \\
53\end{array}$ & $\begin{array}{l}20 \\
19\end{array}$ & $\begin{array}{l}16 \\
14\end{array}$ & $\begin{array}{l}4 \\
5\end{array}$ & $\begin{array}{l}\text { CL-ML } \\
\text { CL-MLL }\end{array}$ & $\begin{array}{l}0.95 \\
0.62\end{array}$ & $=$ \\
\hline $\operatorname{TS} 19$ & 5057111 & 252102 & Low & $\begin{array}{l}\text { Bot tomland } \\
\text { flat }\end{array}$ & 0 & $\begin{array}{l}\text { Short-grass } \\
\text { prafrie }\end{array}$ & Cultivated & $\begin{array}{l}0-6 \\
6-12\end{array}$ & $\begin{array}{l}59 \\
49\end{array}$ & $\begin{array}{l}2 B \\
33\end{array}$ & $\begin{array}{l}13 \\
18\end{array}$ & $\begin{array}{l}\text { SL } \\
\text { L }\end{array}$ & $\begin{array}{l}47 \\
57\end{array}$ & $\begin{array}{l}19 \\
24\end{array}$ & $\begin{array}{l}11 \\
12\end{array}$ & $\begin{array}{r}8 \\
12\end{array}$ & $\begin{array}{l}\text { SC } \\
\text { CL }\end{array}$ & $\begin{array}{l}0.95 \\
0.70\end{array}$ & $\overline{2.64}$ \\
\hline TS 20 & 5057 III & 327103 & $\mathrm{H}$ Igh $_{\mathrm{g}}$ & $\begin{array}{l}\text { Terrace } \\
\text { flat }\end{array}$ & 3 & $\begin{array}{l}\text { Short-grass } \\
\text { prairte }\end{array}$ & $\begin{array}{l}\text { Cultivated } \\
\text { (idle) }\end{array}$ & $\begin{array}{l}0-6 \\
6-12\end{array}$ & $\begin{array}{l}17 \\
16\end{array}$ & $\begin{array}{l}43 \\
41\end{array}$ & $\begin{array}{l}40 \\
43\end{array}$ & $\begin{array}{l}\text { Sic } \\
\text { Sic }\end{array}$ & $\begin{array}{l}86 \\
89\end{array}$ & $\begin{array}{l}67 \\
68\end{array}$ & $\begin{array}{l}31 \\
29\end{array}$ & $\begin{array}{l}36 \\
39\end{array}$ & $\begin{array}{l}\mathrm{CH} \\
\mathrm{CH}\end{array}$ & $\begin{array}{l}3.54 \\
2.75\end{array}$ & $-\overline{2.69}$ \\
\hline & & & & & & & Lop Buri Area & & & & & & & & & & & & \\
\hline PD2S2 & S155II & 937287 & Low & $\begin{array}{l}\text { Bottomland } \\
\text { flat }\end{array}$ & 0 & $\begin{array}{l}\text { Low scrub } \\
\text { savanna }\end{array}$ & Cu1tivated & $\begin{array}{l}0-6 \\
6-12\end{array}$ & $\begin{array}{l}12 \\
11\end{array}$ & $\begin{array}{l}50 \\
47\end{array}$ & $\begin{array}{l}38 \\
42\end{array}$ & $\begin{array}{l}\text { SiCl } \\
\text { SiC }\end{array}$ & $\begin{array}{l}95 \\
93\end{array}$ & $\begin{array}{l}78 \\
88\end{array}$ & $\begin{array}{l}45 \\
53\end{array}$ & $\begin{array}{l}33 \\
35\end{array}$ & $\begin{array}{l}\mathrm{MH} \\
\mathrm{CH}\end{array}$ & $\begin{array}{l}3.27 \\
3.00\end{array}$ & 2.69 \\
\hline PD253 & $51551 I$ & 943282 & High & $\begin{array}{l}\text { Terrace } \\
\text { flat }\end{array}$ & 1 & $\begin{array}{l}\text { Short-8rass } \\
\text { prafre }\end{array}$ & Lawn & $\begin{array}{l}0-6 \\
6-12\end{array}$ & $\begin{array}{l}12 \\
10\end{array}$ & $\begin{array}{l}49 \\
46\end{array}$ & $\begin{array}{l}39 \\
44\end{array}$ & $\begin{array}{l}\text { SiCl } \\
\text { S1C }\end{array}$ & $\begin{array}{l}93 \\
96\end{array}$ & $\begin{array}{l}69 \\
72\end{array}$ & $\begin{array}{l}30 \\
33\end{array}$ & $\begin{array}{l}39 \\
39\end{array}$ & $\begin{array}{l}\mathrm{CH} \\
\mathrm{CH}\end{array}$ & $\begin{array}{l}3.27 \\
2.75\end{array}$ & -- \\
\hline PD254 & $5155 \mathrm{II}$ & 951285 & High & $\begin{array}{l}\text { Lower } \\
\text { slope }\end{array}$ & 6 & $\begin{array}{l}\text { Low scrub } \\
\text { savanna }\end{array}$ & Cultivated & $\begin{array}{l}0-6 \\
6-12\end{array}$ & $\begin{array}{l}12 \\
14\end{array}$ & $\begin{array}{l}39 \\
51\end{array}$ & $\begin{array}{l}49 \\
35\end{array}$ & $\stackrel{\mathrm{C}}{\text { SiCL }}$ & $\begin{array}{l}88 \\
93\end{array}$ & $\begin{array}{l}53 \\
63\end{array}$ & $\begin{array}{l}30 \\
31\end{array}$ & $\begin{array}{l}23 \\
32\end{array}$ & $\begin{array}{l}\mathrm{MH} \\
\mathrm{CH}\end{array}$ & $\begin{array}{l}2.87 \\
2.08\end{array}$ & -- \\
\hline PD255 & $5254 \mathrm{IV}$ & 213155 & Low & $\begin{array}{l}\text { Bottomland } \\
\text { flat }\end{array}$ & 1 & $\begin{array}{l}\text { Short-grass } \\
\text { pratrie }\end{array}$ & Grazed & $\begin{array}{l}0-6 \\
6-12\end{array}$ & $\begin{array}{l}28 \\
30\end{array}$ & $\begin{array}{l}47 \\
46\end{array}$ & $\begin{array}{l}25 \\
24\end{array}$ & $\begin{array}{l}\mathbf{L} \\
\mathrm{L}\end{array}$ & $\begin{array}{l}83 \\
84\end{array}$ & $\begin{array}{l}36 \\
36\end{array}$ & $\begin{array}{l}18 \\
24\end{array}$ & $\begin{array}{l}18 \\
12\end{array}$ & $\begin{array}{l}\mathrm{CL} \\
\mathrm{CL}\end{array}$ & $\begin{array}{l}1.65 \\
1.77\end{array}$ & $\overline{2.63}$ \\
\hline PD256 & $52541 \mathrm{~V}$ & 220162 & Low & $\begin{array}{l}\text { Terrace } \\
\text { flat }\end{array}$ & 1 & $\begin{array}{l}\text { Short-grass } \\
\text { prairie }\end{array}$ & Grazed & $\begin{array}{l}0-6 \\
6-12\end{array}$ & $\begin{array}{l}28 \\
27\end{array}$ & $\begin{array}{l}38 \\
37\end{array}$ & $\begin{array}{l}34 \\
36\end{array}$ & $\begin{array}{l}\mathrm{CL} \\
\mathrm{CL}\end{array}$ & $\begin{array}{l}83 \\
84\end{array}$ & $\begin{array}{l}58 \\
62\end{array}$ & $\begin{array}{l}25 \\
26\end{array}$ & $\begin{array}{l}33 \\
36\end{array}$ & $\begin{array}{l}\mathrm{CH} \\
\mathrm{CH}\end{array}$ & $\begin{array}{l}2.23 \\
1.77\end{array}$ & $\overline{2.71}$ \\
\hline $\operatorname{TS} 21$ & $5155 \mathrm{IV}$ & 771468 & Low & $\begin{array}{l}\text { Bot tonl and } \\
\text { f1at }\end{array}$ & 1 & $\begin{array}{l}\text { Short-grass } \\
\text { prairie }\end{array}$ & $\begin{array}{l}\text { Cultivated } \\
\text { (idle) }\end{array}$ & $\begin{array}{l}0-6 \\
6-12\end{array}$ & $\begin{array}{l}30 \\
30\end{array}$ & $\begin{array}{l}36 \\
33\end{array}$ & $\begin{array}{l}34 \\
37\end{array}$ & $\begin{array}{l}\text { CL } \\
\text { CL }\end{array}$ & $\begin{array}{l}78 \\
79\end{array}$ & $\begin{array}{l}53 \\
55\end{array}$ & $\begin{array}{l}18 \\
18\end{array}$ & $\begin{array}{l}35 \\
37\end{array}$ & $\begin{array}{l}\mathrm{CH} \\
\mathrm{CH}\end{array}$ & $\begin{array}{l}1.25 \\
1.05\end{array}$ & 2.70 \\
\hline TS22 & $5155 \mathrm{IV}$ & 794479 & Low & $\begin{array}{l}\text { Bot tomland } \\
\text { flat }\end{array}$ & 1 & $\begin{array}{l}\text { Short-grass } \\
\text { prafrie }\end{array}$ & $\begin{array}{l}\text { Cultivated } \\
\text { (1dle) }\end{array}$ & $\begin{array}{l}0-6 \\
6-12\end{array}$ & $\begin{array}{l}36 \\
34\end{array}$ & $\begin{array}{l}33 \\
34\end{array}$ & $\begin{array}{l}31 \\
32\end{array}$ & $\begin{array}{l}\mathrm{CL} \\
\mathrm{CL}\end{array}$ & $\begin{array}{l}74 \\
78\end{array}$ & $\begin{array}{l}49 \\
47\end{array}$ & $\begin{array}{l}20 \\
17\end{array}$ & $\begin{array}{l}29 \\
30\end{array}$ & $\begin{array}{l}\mathrm{CL} \\
\mathrm{CL}\end{array}$ & $\begin{array}{l}1.05 \\
1.05\end{array}$ & $\begin{array}{l}-- \\
--\end{array}$ \\
\hline $\operatorname{TS} 23$ & 515SIV & 808487 & Low & $\begin{array}{l}\text { Bottomland } \\
\text { flat }\end{array}$ & 1 & $\begin{array}{l}\text { Law scrub } \\
\text { savanna }\end{array}$ & $\begin{array}{l}\text { Cultivated } \\
\text { (idle) }\end{array}$ & $\begin{array}{l}0-6 \\
6-12\end{array}$ & $\begin{array}{l}31 \\
27\end{array}$ & $\begin{array}{l}41 \\
39\end{array}$ & $\begin{array}{l}28 \\
34\end{array}$ & $\begin{array}{l}\text { CL } \\
\text { CL }\end{array}$ & $\begin{array}{l}79 \\
83\end{array}$ & $\begin{array}{l}42 \\
51\end{array}$ & $\begin{array}{l}19 \\
18\end{array}$ & $\begin{array}{l}23 \\
33\end{array}$ & $\begin{array}{l}\mathrm{CL} \\
\mathrm{CH}\end{array}$ & $\begin{array}{l}1.55 \\
1.15\end{array}$ & $\begin{array}{l}-- \\
2.68\end{array}$ \\
\hline TS24 & $5155 \mathrm{IV}$ & 834489 & Low & $\begin{array}{l}\text { Lower } \\
\text { slope }\end{array}$ & 3 & Savanna & Undisturbed & $\begin{array}{l}0-6 \\
6-12\end{array}$ & $\begin{array}{l}43 \\
41\end{array}$ & $\begin{array}{l}32 \\
28\end{array}$ & $\begin{array}{l}25 \\
31\end{array}$ & $\begin{array}{l}\mathbf{L} \\
\mathrm{CL}\end{array}$ & $\begin{array}{l}70 \\
70\end{array}$ & $\begin{array}{l}37 \\
41\end{array}$ & $\begin{array}{l}21 \\
23\end{array}$ & $\begin{array}{l}16 \\
18\end{array}$ & $\begin{array}{l}\mathrm{CL} \\
\mathrm{CL}\end{array}$ & $\begin{array}{l}2.60 \\
1.65\end{array}$ & $\overline{--}$ \\
\hline TS25 & $5154 \mathrm{I}$ & 071039 & Low & $\begin{array}{l}\text { Terrace } \\
\text { f1at }\end{array}$ & 0 & $\begin{array}{l}\text { Short-grass } \\
\text { prairie }\end{array}$ & Cultivated & $\begin{array}{l}0-6 \\
6-12\end{array}$ & $\begin{array}{r}9 \\
12\end{array}$ & $\begin{array}{l}84 \\
78\end{array}$ & $\begin{array}{r}7 \\
10\end{array}$ & $\begin{array}{l}S_{i} \\
S_{i L}\end{array}$ & $\begin{array}{l}96 \\
94\end{array}$ & $\begin{array}{l}17 \\
16\end{array}$ & $\begin{array}{l}17 \\
16\end{array}$ & $\begin{array}{l}0 \\
0\end{array}$ & $\begin{array}{l}\mathrm{ML} \\
\mathrm{ML}\end{array}$ & $\begin{array}{l}0.85 \\
0.62\end{array}$ & $\overline{2.60}$ \\
\hline $\operatorname{TS} 25 \mathrm{~A}$ & $5254 \mathrm{II}$ & 084012 & Low & $\begin{array}{l}\text { Terrace } \\
\text { flat }\end{array}$ & 0 & Savanna & $\begin{array}{l}\text { Cultivated } \\
\text { (idle) }\end{array}$ & $\begin{array}{l}0-6 \\
6-12\end{array}$ & $\begin{array}{l}30 \\
29\end{array}$ & $\begin{array}{l}58 \\
51\end{array}$ & $\begin{array}{l}12 \\
20\end{array}$ & $\begin{array}{l}\text { SiL } \\
\text { SiL }\end{array}$ & $\begin{array}{l}78 \\
80\end{array}$ & $\begin{array}{l}27 \\
21\end{array}$ & $\begin{array}{l}22 \\
19\end{array}$ & $\begin{array}{l}5 \\
2\end{array}$ & $\begin{array}{l}\mathrm{ML} \\
\mathrm{ML}\end{array}$ & $\begin{array}{l}1.05 \\
0.62\end{array}$ & -- \\
\hline TS25B & $5154 \mathrm{II}$ & 084013 & Low & $\begin{array}{l}\text { Terrace } \\
\text { flat }\end{array}$ & 0 & $\begin{array}{l}\text { Tall scrub } \\
\text { savanna }\end{array}$ & Grazed & $\begin{array}{l}0-6 \\
6-12\end{array}$ & $\begin{array}{l}42 \\
43\end{array}$ & $\begin{array}{l}42 \\
41\end{array}$ & $\begin{array}{l}16 \\
16\end{array}$ & $\begin{array}{l}\mathrm{L} \\
\mathrm{L}\end{array}$ & $\begin{array}{l}66 \\
44\end{array}$ & $\begin{array}{l}29 \\
23\end{array}$ & $\begin{array}{l}22 \\
17\end{array}$ & $\begin{array}{l}7 \\
6\end{array}$ & $\underset{S M-S C}{M L}$ & $\begin{array}{l}1.05 \\
0.62\end{array}$ & -- \\
\hline TS26 & $5153 \mathrm{I}$ & 91.2764 & Low & $\begin{array}{l}\text { Bot tomland } \\
\text { flat }\end{array}$ & 0 & $\begin{array}{l}\text { Short-grass } \\
\text { pra1rie }\end{array}$ & Cultivated & $\begin{array}{l}0-6 \\
6-12\end{array}$ & $\begin{array}{l}16 \\
13\end{array}$ & $\begin{array}{l}49 \\
49\end{array}$ & $\begin{array}{l}35 \\
38\end{array}$ & $\begin{array}{l}\text { SICL } \\
\text { SICL }\end{array}$ & $\begin{array}{l}80 \\
97\end{array}$ & $\begin{array}{l}75 \\
74\end{array}$ & $\begin{array}{l}38 \\
31\end{array}$ & $\begin{array}{l}37 \\
43\end{array}$ & $\begin{array}{l}\mathrm{MH} \\
\mathrm{CH}\end{array}$ & $\begin{array}{l}5.20 \\
2.08\end{array}$ & -- \\
\hline & & & & & & & Bangtok Area & & & & & & & & & & & & \\
\hline PD244 & $515211 \mathrm{I}$ & 740113 & Low & $\begin{array}{l}\text { Bottomland } \\
\text { depresston }\end{array}$ & 0 & $\begin{array}{l}\text { Short-grass } \\
\text { prairie }\end{array}$ & $\begin{array}{l}\text { Cultivated } \\
\text { (Idle) }\end{array}$ & $\begin{array}{l}0-6 \\
6-12\end{array}$ & $\begin{array}{l}6 \\
6\end{array}$ & $\begin{array}{l}41 \\
37\end{array}$ & $\begin{array}{l}53 \\
57\end{array}$ & $\begin{array}{l}\text { Sic } \\
\text { C }\end{array}$ & $\begin{array}{l}99 \\
99\end{array}$ & $\begin{array}{l}72 \\
67\end{array}$ & $\begin{array}{l}27 \\
23\end{array}$ & $\begin{array}{l}45 \\
44\end{array}$ & $\begin{array}{l}\mathrm{CH} \\
\mathrm{CH}\end{array}$ & $\begin{array}{l}1.98 \\
1.55\end{array}$ & $\overline{2.68}$ \\
\hline PD245 & $5152 \mathrm{IV}$ & 711319 & Low & $\begin{array}{l}\text { Bot tomland } \\
\text { flat }\end{array}$ & 1 & $\begin{array}{l}\text { Short-grass } \\
\text { prairie }\end{array}$ & Cultivated & $\begin{array}{l}0-6 \\
6-12\end{array}$ & $\begin{array}{l}13 \\
11\end{array}$ & $\begin{array}{l}41 \\
41\end{array}$ & $\begin{array}{l}48 \\
48\end{array}$ & $\begin{array}{l}\text { SiC } \\
\text { Sic }\end{array}$ & $\begin{array}{l}98 \\
98\end{array}$ & $\begin{array}{l}67 \\
66\end{array}$ & $\begin{array}{l}29 \\
27\end{array}$ & $\begin{array}{l}38 \\
39\end{array}$ & $\begin{array}{l}\mathrm{CH} \\
\mathrm{CB}\end{array}$ & $\begin{array}{l}3.00 \\
1.65\end{array}$ & $\begin{array}{l}2.68 \\
--\end{array}$ \\
\hline TS8 & $5052 \mathrm{II}$ & 592229 & Low & $\begin{array}{l}\text { Bottomland } \\
\text { flat }\end{array}$ & 0 & $\begin{array}{l}\text { Short-grass } \\
\text { prairie }\end{array}$ & Cultivated & $\begin{array}{l}0-6 \\
6-12\end{array}$ & $\begin{array}{l}13 \\
12\end{array}$ & $\begin{array}{l}43 \\
41\end{array}$ & $\begin{array}{l}44 \\
47\end{array}$ & $\begin{array}{l}\text { Sic } \\
\text { Sic }\end{array}$ & $\begin{array}{l}94 \\
94\end{array}$ & $\begin{array}{l}65 \\
66\end{array}$ & $\begin{array}{l}29 \\
29\end{array}$ & $\begin{array}{l}36 \\
37\end{array}$ & $\begin{array}{l}\mathrm{CH} \\
\mathrm{CH}\end{array}$ & $\begin{array}{l}2.08 \\
1.45\end{array}$ & $-\overline{2.71}$ \\
\hline & & & & & & & Pran Buri Ax & & & & & & & & & & & & \\
\hline PD257 & $4948 \mathrm{I}$ & 040904 & High & $\begin{array}{l}\text { Terrace } \\
\text { flat }\end{array}$ & 1 & $\begin{array}{l}\text { Short-grass } \\
\text { prairle }\end{array}$ & Lawn & $\begin{array}{l}0-6 \\
6-12\end{array}$ & $\begin{array}{l}72 \\
75\end{array}$ & $\begin{array}{l}23 \\
19\end{array}$ & $\begin{array}{l}5 \\
6\end{array}$ & $\begin{array}{l}\text { SL } \\
\text { SL }\end{array}$ & $\begin{array}{l}37 \\
33\end{array}$ & $\overline{-}$ & $\overline{--}$ & $\begin{array}{l}\mathrm{NP} \\
\mathrm{NP}\end{array}$ & $\begin{array}{l}\text { SM } \\
\text { SM }\end{array}$ & $\begin{array}{l}0.70 \\
0.55\end{array}$ & -- \\
\hline $\operatorname{TS} 29$ & $49481 \mathrm{I}$ & 062776 & Low & $\begin{array}{l}\text { Bottomland } \\
\text { f1at }\end{array}$ & 1 & $\begin{array}{l}\text { Short-grass } \\
\text { prafrie }\end{array}$ & $\begin{array}{l}\text { Cultivated } \\
\text { (idle) }\end{array}$ & $\begin{array}{l}0-6 \\
6-12\end{array}$ & $\begin{array}{l}68 \\
54\end{array}$ & $\begin{array}{l}21 \\
17\end{array}$ & $\begin{array}{l}11 \\
29\end{array}$ & $\begin{array}{l}\text { SL } \\
\text { SCL }\end{array}$ & $\begin{array}{l}45 \\
49\end{array}$ & $\begin{array}{l}18 \\
28\end{array}$ & $\begin{array}{l}13 \\
11\end{array}$ & $\begin{array}{r}5 \\
17\end{array}$ & $\begin{array}{l}\text { SM-SC } \\
\text { SC }\end{array}$ & $\begin{array}{l}0.78 \\
0.55\end{array}$ & 2.63 \\
\hline TS 30 & $4948 \mathrm{II}$ & 053772 & 81gh & $\begin{array}{l}\text { Terrace } \\
\text { slope }\end{array}$ & 3 & $\begin{array}{l}\text { Tall scrub } \\
\text { savanna }\end{array}$ & Cu1tivated & $\begin{array}{l}0-6 \\
6-12\end{array}$ & $\begin{array}{l}64 \\
62\end{array}$ & $\begin{array}{l}24 \\
24\end{array}$ & $\begin{array}{l}12 \\
12\end{array}$ & $\begin{array}{l}\text { SL } \\
\text { SL }\end{array}$ & $\begin{array}{l}51 \\
48\end{array}$ & $\begin{array}{l}18 \\
17\end{array}$ & $\begin{array}{l}15 \\
13\end{array}$ & $\begin{array}{l}3 \\
4\end{array}$ & $\underset{\mathrm{SM}-\mathrm{SC}}{\mathrm{ML}}$ & $\begin{array}{l}1.05 \\
0.62\end{array}$ & $\overline{2.67}$ \\
\hline TS31 & $4948 \mathrm{II}$ & 951732 & High & $\begin{array}{l}\text { Terrace } \\
\text { flat }\end{array}$ & 0 & $\begin{array}{l}\text { Tall serub } \\
\text { wood land }\end{array}$ & Und ist urbed & $\begin{array}{l}0-6 \\
6-12\end{array}$ & $\begin{array}{l}44 \\
38\end{array}$ & $\begin{array}{l}45 \\
44\end{array}$ & $\begin{array}{l}11 \\
18\end{array}$ & $\begin{array}{l}\mathbf{L} \\
\mathbf{L}\end{array}$ & $\begin{array}{l}70 \\
73\end{array}$ & $\begin{array}{l}21 \\
20\end{array}$ & $\begin{array}{l}15 \\
13\end{array}$ & $\begin{array}{l}6 \\
7\end{array}$ & $\begin{array}{l}\text { CL-ML } \\
\text { CL-ML }\end{array}$ & $\begin{array}{l}1.33 \\
0.78\end{array}$ & -- \\
\hline TS32 & $4948 \mathrm{II}$ & 944730 & High & $\begin{array}{l}\text { Terrace } \\
\text { flat }\end{array}$ & 1 & $\begin{array}{l}\text { Tall-grass } \\
\text { prairie }\end{array}$ & Undisturbed & $\begin{array}{l}0-6 \\
6-12\end{array}$ & $\begin{array}{l}48 \\
43\end{array}$ & $\begin{array}{l}40 \\
43\end{array}$ & $\begin{array}{l}12 \\
14\end{array}$ & $\begin{array}{l}\mathrm{L} \\
\mathrm{L}\end{array}$ & $\begin{array}{l}64 \\
66\end{array}$ & $\begin{array}{l}18 \\
17\end{array}$ & $\begin{array}{l}14 \\
12\end{array}$ & $\begin{array}{l}4 \\
5\end{array}$ & $\underset{C L-M L}{C L-M L}$ & $\begin{array}{l}0.95 \\
0.78\end{array}$ & -- \\
\hline TS33 & $4948 \mathrm{II}$ & 925730 & High & $\begin{array}{l}\text { Terrace } \\
\text { flat }\end{array}$ & 0 & Low scrub & Und Isturbed & $\begin{array}{l}0-6 \\
6-12\end{array}$ & $\begin{array}{l}27 \\
30\end{array}$ & $\begin{array}{l}55 \\
46\end{array}$ & $\begin{array}{l}18 \\
24\end{array}$ & $\begin{array}{l}\text { SiL } \\
\text { L }\end{array}$ & $\begin{array}{l}85 \\
86\end{array}$ & $\begin{array}{l}28 \\
23\end{array}$ & $\begin{array}{l}19 \\
16\end{array}$ & $\begin{array}{l}9 \\
7\end{array}$ & $\underset{\mathrm{CL}-\mathrm{ML}}{\mathrm{CL}}$ & $\begin{array}{l}1.65 \\
0.95\end{array}$ & -- \\
\hline TS 34 & 4948 II & 967679 & High & $\begin{array}{l}\text { Terrace } \\
\text { flat }\end{array}$ & 1 & $\begin{array}{l}\text { Short-grass } \\
\text { prairie }\end{array}$ & $\begin{array}{l}\text { Cultivated } \\
\text { (Idle) }\end{array}$ & $\begin{array}{l}0-6 \\
6-12\end{array}$ & $\begin{array}{l}39 \\
34\end{array}$ & $\begin{array}{l}43 \\
39\end{array}$ & $\begin{array}{l}18 \\
27\end{array}$ & $\begin{array}{l}\text { L } \\
\text { L }\end{array}$ & $\begin{array}{l}76 \\
80\end{array}$ & $\begin{array}{l}26 \\
27\end{array}$ & $\begin{array}{l}15 \\
14\end{array}$ & $\begin{array}{r}9 \\
13\end{array}$ & $\begin{array}{l}\mathrm{CL} \\
\mathrm{CL}\end{array}$ & $\begin{array}{l}1.65 \\
1.15\end{array}$ & $\overline{2.65}$ \\
\hline
\end{tabular}




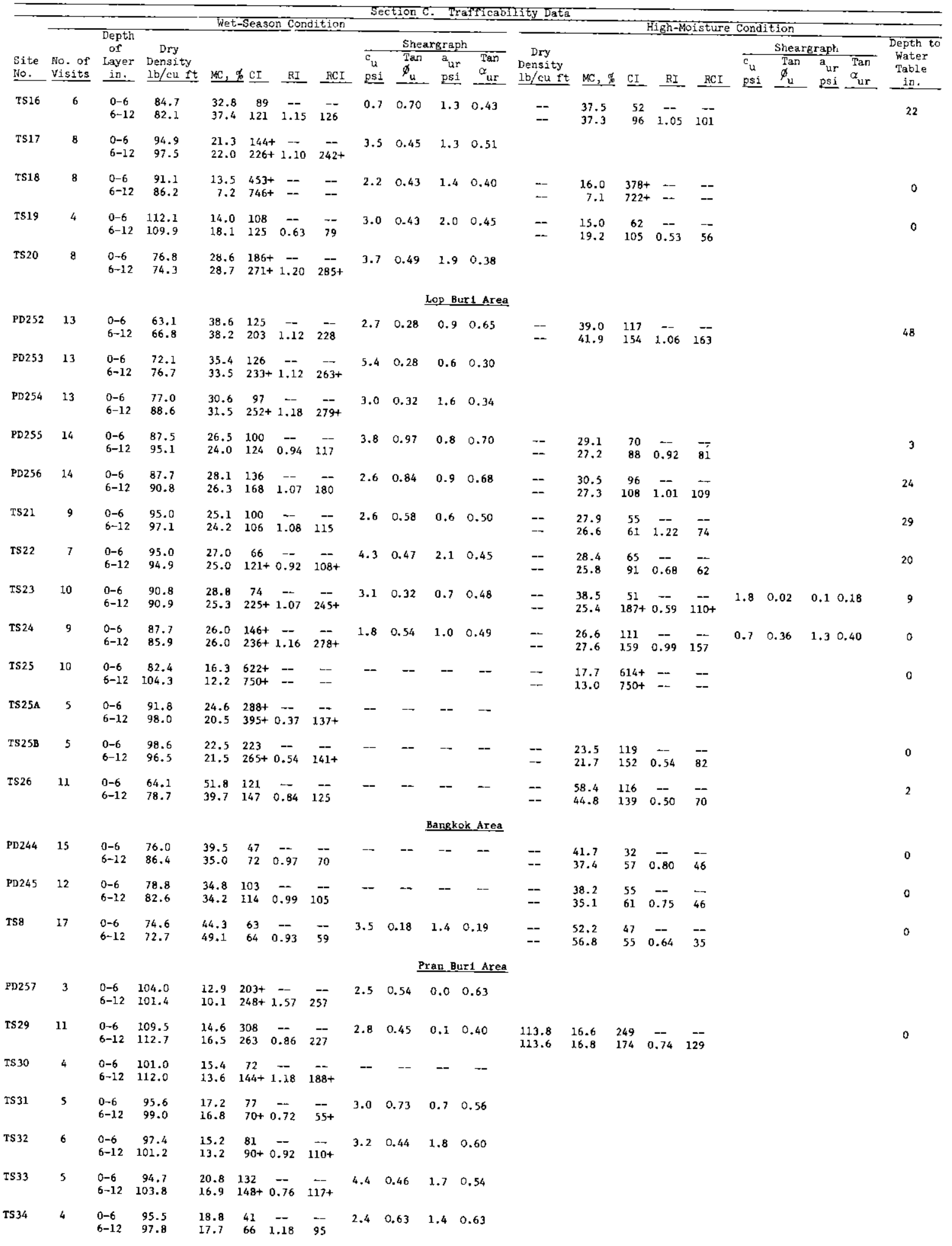




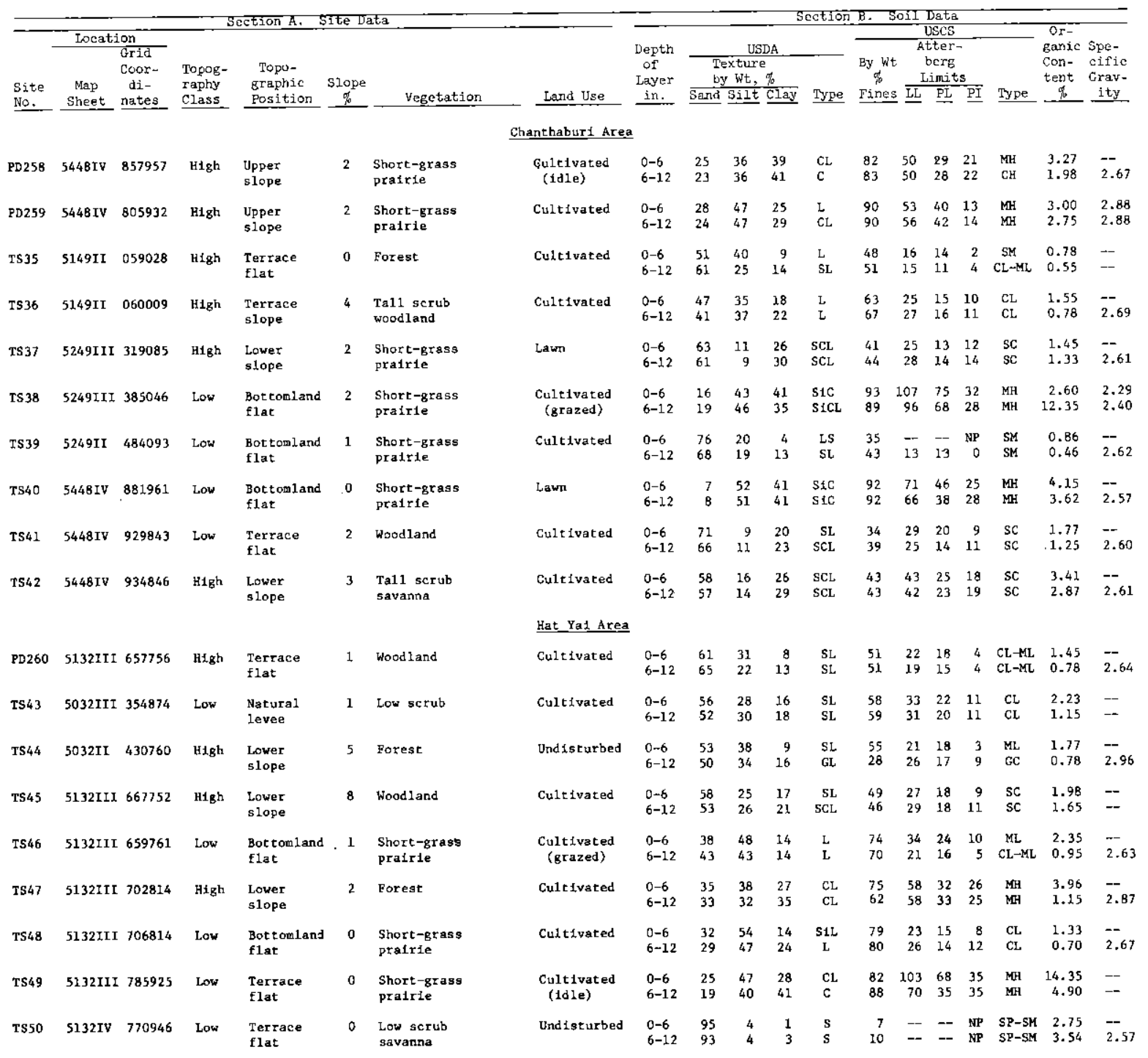




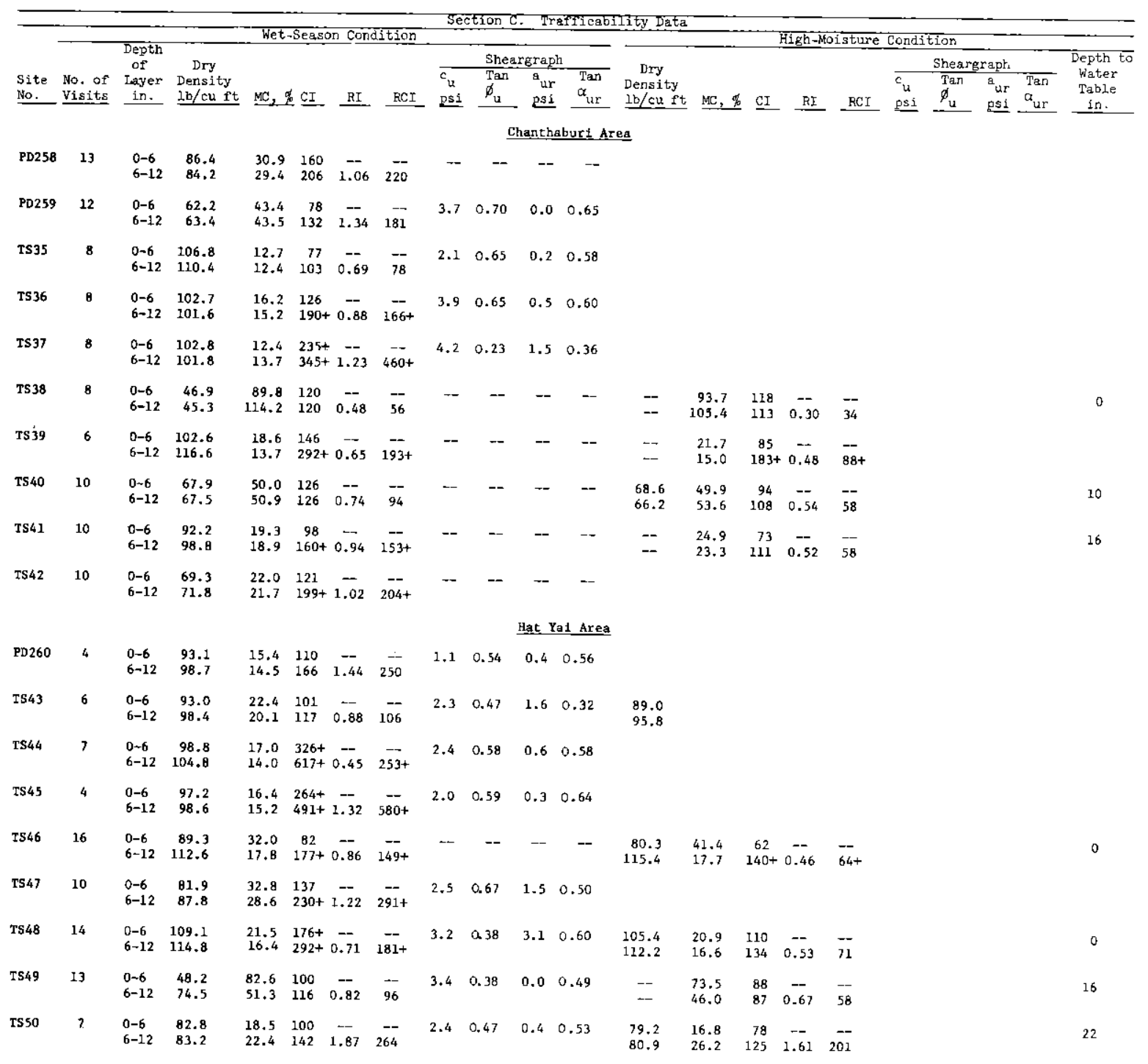


Table A5

CRREL Airphoto Pattern Study

Sumary of Site, SoIl, and Trafficability Data

\begin{tabular}{|c|c|c|c|c|c|c|c|c|c|c|c|c|c|c|c|c|c|c|c|}
\hline \multirow{4}{*}{$\begin{array}{l}\text { Site } \\
\text { Bo. }\end{array}$} & \multirow{2}{*}{\multicolumn{2}{|c|}{ Location }} & \multirow{4}{*}{$\begin{array}{l}\text { Topog- } \\
\text { raphy } \\
\text { Class } \\
\end{array}$} & ection A: Si & Ite Data & \multirow[b]{4}{*}{ Vegetation } & \multirow[b]{4}{*}{ Land Use } & \multirow{4}{*}{$\begin{array}{l}\text { Depth } \\
\text { of } \\
\text { Layex } \\
\text { in. } \\
\end{array}$} & \multirow{2}{*}{\multicolumn{9}{|c|}{ Section B. Soil Data }} & \multirow{4}{*}{$\begin{array}{l}\text { Or- } \\
\text { ganic } \\
\text { Con- } \\
\text { tent } \\
\% \\
\end{array}$} & \multirow{4}{*}{$\begin{array}{l}\text { Spe- } \\
\text { cific } \\
\text { Grav- } \\
\text { ity } \\
\end{array}$} \\
\hline & & & & \multirow{3}{*}{$\begin{array}{l}\text { Topo- } \\
\text { graphic } \\
\text { Position } \\
\end{array}$} & \multirow{3}{*}{$\begin{array}{c}\text { Slope } \\
\not \\
\end{array}$} & & & & \multirow{3}{*}{\multicolumn{3}{|c|}{$\begin{array}{c}\frac{\text { USDA }}{\text { Texture }} \\
\text { by wit, } \\
\text { Sand Silt clay }\end{array}$}} & & & \multicolumn{2}{|c|}{ Atter- } & & & & \\
\hline & Map & $\begin{array}{l}\text { Coor- } \\
\text { di- }\end{array}$ & & & & & & & & & & \multirow[b]{2}{*}{ Type* } & \multirow{2}{*}{$\begin{array}{l}\text { By Wt } \\
\% \\
\text { Fines } \\
\end{array}$} & \multirow{2}{*}{\multicolumn{3}{|c|}{ 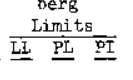 }} & \multirow[b]{2}{*}{ Type } & & \\
\hline & Shcet & nates & & & & & & & & & & & & & & & & & \\
\hline & & & & & & & Bur1 Area & & & & & & & & & & & & \\
\hline 1 & $5154 \mathrm{I}$ & 946123 & Low & $\begin{array}{l}\text { Bottonland } \\
\text { flat }\end{array}$ & -- & -- & Cultivated & $\begin{array}{l}0-6 \\
6-12\end{array}$ & $\begin{array}{l}9 \\
9\end{array}$ & $\begin{array}{l}65 \\
66\end{array}$ & $\begin{array}{l}26 \\
25\end{array}$ & $\begin{array}{l}\text { SiL } \\
\text { SIL }\end{array}$ & $\begin{array}{l}94 \\
93\end{array}$ & $\begin{array}{l}44 \\
57\end{array}$ & $\begin{array}{l}21 \\
22\end{array}$ & $\begin{array}{l}23 \\
35\end{array}$ & $\begin{array}{l}\mathrm{CL} \\
\mathrm{CH}\end{array}$ & $\begin{array}{l}0.92 \\
0.74\end{array}$ & $\begin{array}{l}2.68 \\
2.65\end{array}$ \\
\hline 2 & $5153 \mathrm{IV}$ & 820785 & Low & $\begin{array}{l}\text { Bottomland } \\
\text { flat }\end{array}$ & -- & -- & $\begin{array}{l}\text { Cultivated } \\
\text { (Idle) }\end{array}$ & $\begin{array}{l}0-6 \\
6-12\end{array}$ & $\begin{array}{l}5 \\
7\end{array}$ & $\begin{array}{l}67 \\
67\end{array}$ & $\begin{array}{l}28 \\
26\end{array}$ & $\begin{array}{r}\text { SiCL } \\
51 L\end{array}$ & $\begin{array}{l}97 \\
95\end{array}$ & $\begin{array}{l}57 \\
69\end{array}$ & $\begin{array}{l}29 \\
25\end{array}$ & $\begin{array}{l}28 \\
44\end{array}$ & $\begin{array}{l}\mathrm{CH} \\
\mathrm{CH}\end{array}$ & $\begin{array}{l}2.41 \\
0.97\end{array}$ & $\begin{array}{l}2.65 \\
2.74\end{array}$ \\
\hline 4 & $5155 \mathrm{II}$ & 992256 & H1gh & Upland Elat & -- & - & Cultivated & $\begin{array}{l}0-6 \\
6-12\end{array}$ & $\begin{array}{l}48 \\
48\end{array}$ & $\begin{array}{l}39 \\
39\end{array}$ & $\begin{array}{l}13 \\
13\end{array}$ & $\begin{array}{l}\mathrm{L} \\
\mathrm{L}\end{array}$ & $\begin{array}{l}60 \\
60\end{array}$ & $\begin{array}{l}48 \\
48\end{array}$ & $\begin{array}{l}25 \\
25\end{array}$ & $\begin{array}{l}23 \\
23\end{array}$ & $\begin{array}{l}\mathrm{CL} \\
\mathrm{CL}\end{array}$ & $\begin{array}{l}3.41 \\
3.41\end{array}$ & $\begin{array}{l}2.69 \\
2.69\end{array}$ \\
\hline 5 & 515511 & 923265 & Low & $\begin{array}{l}\text { Bottom1and } \\
\text { flat }\end{array}$ & -- & -- & $\begin{array}{l}\text { Culrivated } \\
\text { (1dle) }\end{array}$ & $\begin{array}{l}0-6 \\
6-12\end{array}$ & $\begin{array}{l}21 \\
18\end{array}$ & $\begin{array}{l}69 \\
71\end{array}$ & $\begin{array}{l}10 \\
11\end{array}$ & $\begin{array}{l}\text { SiL } \\
\text { SIL }\end{array}$ & $\begin{array}{l}88 \\
87\end{array}$ & $\begin{array}{l}90 \\
70\end{array}$ & $\begin{array}{l}40 \\
27\end{array}$ & $\begin{array}{l}50 \\
43\end{array}$ & $\begin{array}{l}\mathrm{MH} \\
\mathrm{CH}\end{array}$ & $\begin{array}{l}2.67 \\
2.22\end{array}$ & $\begin{array}{l}2.59 \\
2.77\end{array}$ \\
\hline 6 & $5154 \mathrm{IV}$ & 868128 & Low & $\begin{array}{l}\text { Natural } \\
\text { levee }\end{array}$ & -- & -- & Cultivated & $\begin{array}{l}0-6 \\
6-12\end{array}$ & $\begin{array}{l}10 \\
11\end{array}$ & $\begin{array}{l}66 \\
64\end{array}$ & $\begin{array}{l}24 \\
25\end{array}$ & $\begin{array}{l}\text { SiL } \\
\text { SiL }\end{array}$ & $\begin{array}{l}95 \\
93\end{array}$ & $\begin{array}{l}62 \\
59\end{array}$ & $\begin{array}{l}22 \\
28\end{array}$ & $\begin{array}{l}40 \\
31\end{array}$ & $\begin{array}{l}\mathrm{CH} \\
\mathrm{CH}\end{array}$ & $\begin{array}{l}1.55 \\
0.82\end{array}$ & $\begin{array}{l}2.63 \\
2.70\end{array}$ \\
\hline 7 & $5155 \mathrm{II}$ & 960348 & Low & $\begin{array}{l}\text { Bot tomland } \\
\text { flat }\end{array}$ & -- & -- & Cultivated & $\begin{array}{l}0-6 \\
6-12\end{array}$ & $\begin{array}{l}21 \\
11\end{array}$ & $\begin{array}{l}69 \\
81\end{array}$ & $\begin{array}{r}10 \\
8\end{array}$ & $\begin{array}{l}\text { S1L } \\
\text { S1 }\end{array}$ & $\begin{array}{l}88 \\
94\end{array}$ & $\begin{array}{l}79 \\
84\end{array}$ & $\begin{array}{l}37 \\
30\end{array}$ & $\begin{array}{l}42 \\
54\end{array}$ & $\begin{array}{l}\mathrm{MH} \\
\mathrm{CH}\end{array}$ & $\begin{array}{l}2.35 \\
1.82\end{array}$ & $\begin{array}{l}2.74 \\
2.73\end{array}$ \\
\hline 11 & 515SIII & 628387 & Low & $\begin{array}{l}\text { Natural } \\
\text { levee }\end{array}$ & -- & -- & Cultivated & $\begin{array}{l}0-6 \\
6-12\end{array}$ & $\begin{array}{l}10 \\
17\end{array}$ & $\begin{array}{l}63 \\
63\end{array}$ & $\begin{array}{l}27 \\
20\end{array}$ & $\begin{array}{l}\text { Sil } \\
\text { SiL }\end{array}$ & $\begin{array}{l}94 \\
89\end{array}$ & $\begin{array}{l}51 \\
46\end{array}$ & $\begin{array}{l}29 \\
24\end{array}$ & $\begin{array}{l}22 \\
22\end{array}$ & $\begin{array}{l}\text { MH } \\
\text { CL }\end{array}$ & $\begin{array}{l}1.67 \\
1.00\end{array}$ & $\begin{array}{l}2.69 \\
2.70\end{array}$ \\
\hline 14 & $5154 \mathrm{II}$ & 139879 & Low & $\begin{array}{l}\text { Bottomland } \\
\text { flat }\end{array}$ & -- & - & Cultivated & $\begin{array}{l}0-6 \\
6-12\end{array}$ & $\begin{array}{l}6 \\
3\end{array}$ & $\begin{array}{l}81 \\
79\end{array}$ & $\begin{array}{l}13 \\
18\end{array}$ & $\begin{array}{l}\mathrm{SiL} \\
\mathrm{SIL}\end{array}$ & $\begin{array}{l}96 \\
98\end{array}$ & $\begin{array}{l}28 \\
28\end{array}$ & $\begin{array}{l}23 \\
21\end{array}$ & $\begin{array}{l}5 \\
7\end{array}$ & $\underset{C L-M L}{M L}$ & 1.33 & $\begin{array}{l}2.60 \\
2.60\end{array}$ \\
\hline 27 & 51541 & 023087 & Low & $\begin{array}{l}\text { Natural } \\
\text { levee }\end{array}$ & -- & - & Cultivated & $\begin{array}{l}0-6 \\
6-12\end{array}$ & $\begin{array}{l}23 \\
25\end{array}$ & $\begin{array}{l}67 \\
62\end{array}$ & $\begin{array}{l}10 \\
13\end{array}$ & $\begin{array}{l}\text { SIL } \\
\text { SiL }\end{array}$ & $\begin{array}{l}83 \\
81\end{array}$ & $\begin{array}{l}38 \\
47\end{array}$ & $\begin{array}{l}28 \\
20\end{array}$ & $\begin{array}{l}10 \\
27\end{array}$ & $\begin{array}{l}\text { ML } \\
\text { CL }\end{array}$ & $\begin{array}{l}1.15 \\
0.51\end{array}$ & $\begin{array}{l}2.71 \\
2.70\end{array}$ \\
\hline 19 & $5154 \mathrm{I}$ & 045089 & Low & Terrace flat & t - & -- & Culcivated & $\begin{array}{l}0-6 \\
6-12\end{array}$ & $\begin{array}{l}14 \\
13\end{array}$ & $\begin{array}{l}66 \\
65\end{array}$ & $\begin{array}{l}20 \\
22\end{array}$ & $\begin{array}{l}\text { S1L } \\
\text { S\&L }\end{array}$ & $\begin{array}{l}90 \\
92\end{array}$ & $\begin{array}{l}52 \\
54\end{array}$ & $\begin{array}{l}25 \\
24\end{array}$ & $\begin{array}{l}27 \\
30\end{array}$ & $\begin{array}{l}\mathrm{CH} \\
\mathrm{CH}\end{array}$ & $\begin{array}{l}1.00 \\
0.70\end{array}$ & $\begin{array}{l}2.67 \\
2.67\end{array}$ \\
\hline 20 & $5154 \mathrm{r}$ & 048093 & Low & $\begin{array}{l}\text { Natural } \\
\text { levee }\end{array}$ & -- & - & Cultivated & $\begin{array}{l}0-6 \\
6-12\end{array}$ & $\begin{array}{r}20 \\
7\end{array}$ & $\begin{array}{l}67 \\
65\end{array}$ & $\begin{array}{l}13 \\
28\end{array}$ & $\begin{array}{r}\text { SiL } \\
\text { SiCL }\end{array}$ & $\begin{array}{l}85 \\
94\end{array}$ & $\begin{array}{l}35 \\
57\end{array}$ & $\begin{array}{l}22 \\
22\end{array}$ & $\begin{array}{l}13 \\
35\end{array}$ & $\begin{array}{l}\mathrm{CL} \\
\mathrm{CH}\end{array}$ & $\begin{array}{l}1.10 \\
0.74\end{array}$ & $\begin{array}{l}2.72 \\
2.64\end{array}$ \\
\hline 21 & $5154 \mathrm{I}$ & 021130 & Low & $\begin{array}{l}\text { Bottomland } \\
\text { flat }\end{array}$ & -- & - & Cultivated & $\begin{array}{l}0-6 \\
6-12\end{array}$ & $\begin{array}{l}10 \\
44\end{array}$ & $\begin{array}{l}80 \\
50\end{array}$ & $\begin{array}{r}10 \\
6\end{array}$ & $\begin{array}{l}\text { Si } \\
\text { siL }\end{array}$ & $\begin{array}{l}94 \\
59\end{array}$ & $\begin{array}{l}40 \\
32\end{array}$ & $\begin{array}{l}16 \\
22\end{array}$ & $\begin{array}{l}24 \\
10\end{array}$ & $\begin{array}{l}\text { CL } \\
\text { CL }\end{array}$ & $\begin{array}{l}1.15 \\
0.51\end{array}$ & $\begin{array}{l}2.60 \\
2.59\end{array}$ \\
\hline 23 & $5154 \mathrm{I}$ & 053164 & High & Lower slope & - & -- & Cultivated & $\begin{array}{l}0-6 \\
6-12\end{array}$ & $\begin{array}{l}19 \\
17\end{array}$ & $\begin{array}{l}70 \\
66\end{array}$ & $\begin{array}{l}11 \\
17\end{array}$ & $\begin{array}{l}\text { S1L } \\
\text { S1L }\end{array}$ & $\begin{array}{l}87 \\
88\end{array}$ & $\begin{array}{l}45 \\
51\end{array}$ & $\begin{array}{l}16 \\
21\end{array}$ & $\begin{array}{l}29 \\
30\end{array}$ & $\begin{array}{l}\mathrm{CL} \\
\mathrm{CH}\end{array}$ & $\begin{array}{l}1.15 \\
0.59\end{array}$ & $\begin{array}{l}2.62 \\
2.71\end{array}$ \\
\hline 27 & $5154 \mathrm{III}$ & 623946 & Low & $\begin{array}{l}\text { Natural } \\
\text { levee }\end{array}$ & -- & - & Cultivated & $\begin{array}{l}0-6 \\
6-12\end{array}$ & $\begin{array}{l}14 \\
13\end{array}$ & $\begin{array}{l}68 \\
71\end{array}$ & $\begin{array}{l}18 \\
16\end{array}$ & $\begin{array}{l}\text { SIL } \\
\text { SIL }\end{array}$ & $\begin{array}{l}91 \\
92\end{array}$ & $\begin{array}{l}48 \\
39\end{array}$ & $\begin{array}{l}25 \\
24\end{array}$ & $\begin{array}{l}23 \\
15\end{array}$ & $\begin{array}{l}\mathrm{CL} \\
\mathrm{CL}\end{array}$ & $\begin{array}{l}1.10 \\
1.05\end{array}$ & $\begin{array}{l}2.74 \\
2.73\end{array}$ \\
\hline 28 & 5154111 & 657860 & Low & $\begin{array}{l}\text { Bottomland } \\
\text { flat }\end{array}$ & -- & -- & Und isturbed & $\begin{array}{l}0-6 \\
6-12\end{array}$ & $\begin{array}{r}9 \\
13\end{array}$ & $\begin{array}{l}66 \\
62\end{array}$ & $\begin{array}{l}25 \\
25\end{array}$ & $\begin{array}{l}\text { SiL } \\
\text { SIL }\end{array}$ & $\begin{array}{l}95 \\
92\end{array}$ & $\begin{array}{l}58 \\
53\end{array}$ & $\begin{array}{l}26 \\
27\end{array}$ & $\begin{array}{l}32 \\
26\end{array}$ & $\begin{array}{l}\mathrm{CH} \\
\mathrm{CH}\end{array}$ & $\begin{array}{l}2.94 \\
1.45\end{array}$ & $\begin{array}{l}2.72 \\
2.76\end{array}$ \\
\hline 30 & $5153 I$ & 891751 & Low & $\begin{array}{l}\text { Bottomland } \\
\text { flat }\end{array}$ & -- & -- & Cultivated & $\begin{array}{l}0-6 \\
6-12\end{array}$ & $\begin{array}{l}35 \\
25\end{array}$ & $\begin{array}{l}44 \\
50\end{array}$ & $\begin{array}{l}21 \\
25\end{array}$ & $\stackrel{L}{\mathrm{~L}}$ & $\begin{array}{l}70 \\
79\end{array}$ & $\begin{array}{l}63 \\
69\end{array}$ & $\begin{array}{l}24 \\
35\end{array}$ & $\begin{array}{l}39 \\
34\end{array}$ & $\begin{array}{l}\mathrm{CH} \\
\mathrm{MH}\end{array}$ & $\begin{array}{l}3.82 \\
2.16\end{array}$ & $\begin{array}{l}2.68 \\
2.66\end{array}$ \\
\hline 31 & $5153 I$ & 948788 & Low & $\begin{array}{l}\text { Bottomland } \\
\text { flat }\end{array}$ & -- & - & $\begin{array}{l}\text { Cultivated } \\
\text { (1dle) }\end{array}$ & $\begin{array}{l}0-6 \\
6-12\end{array}$ & $\begin{array}{l}16 \\
17\end{array}$ & $\begin{array}{l}62 \\
56\end{array}$ & $\begin{array}{l}22 \\
27\end{array}$ & $\begin{array}{l}\text { SiL } \\
\text { Sit }\end{array}$ & $\begin{array}{l}89 \\
87\end{array}$ & $\begin{array}{l}68 \\
62\end{array}$ & $\begin{array}{l}31 \\
27\end{array}$ & $\begin{array}{l}37 \\
35\end{array}$ & $\begin{array}{l}\mathrm{CH} \\
\mathrm{CH}\end{array}$ & $\begin{array}{l}1.65 \\
0.87\end{array}$ & $\begin{array}{l}2.71 \\
2.69\end{array}$ \\
\hline 33 & 51531 & 963793 & Low & $\begin{array}{l}\text { Bottomland } \\
\text { flat }\end{array}$ & -- & -- & Cultivated & $\begin{array}{l}0-6 \\
6-12\end{array}$ & $\begin{array}{l}18 \\
22\end{array}$ & $\begin{array}{l}54 \\
47\end{array}$ & $\begin{array}{l}28 \\
31\end{array}$ & $\begin{array}{c}S_{1 C L} \\
\mathrm{CL}\end{array}$ & $\begin{array}{l}87 \\
83\end{array}$ & $\begin{array}{l}73 \\
79\end{array}$ & $\begin{array}{l}44 \\
39\end{array}$ & $\begin{array}{l}29 \\
40\end{array}$ & $\begin{array}{l}\text { MH } \\
\text { MH }\end{array}$ & $\begin{array}{l}4.70 \\
4.20\end{array}$ & $\begin{array}{l}2.58 \\
2.59\end{array}$ \\
\hline 37 & 5154 II & 040917 & Low & Terrace flat & $5--$ & -- & Cuitivaced & $\begin{array}{l}0-6 \\
6-12\end{array}$ & $\begin{array}{l}38 \\
41\end{array}$ & $\begin{array}{l}57 \\
43\end{array}$ & $\begin{array}{r}5 \\
16\end{array}$ & $\stackrel{S_{1 L}}{\mathrm{~L}}$ & $\begin{array}{l}74 \\
70\end{array}$ & $\begin{array}{l}17 \\
25\end{array}$ & $\begin{array}{l}16 \\
15\end{array}$ & $\begin{array}{r}1 \\
10\end{array}$ & $\begin{array}{l}\mathrm{ML} \\
\mathrm{CL}\end{array}$ & $\begin{array}{l}0.70 \\
0.63\end{array}$ & $\begin{array}{l}2.68 \\
2.65\end{array}$ \\
\hline 39 & $51541 \mathrm{I}$ & 071927 & Low & Lower slope & -- & -- & Cultivated & $\begin{array}{l}0-6 \\
6-12\end{array}$ & $\begin{array}{l}33 \\
29\end{array}$ & $\begin{array}{l}66 \\
70\end{array}$ & $\begin{array}{l}1 \\
1\end{array}$ & $\begin{array}{l}\text { SiL } \\
\text { SiL }\end{array}$ & $\begin{array}{l}79 \\
81\end{array}$ & $\overline{19}$ & $\overline{19}$ & $\begin{array}{r}\text { NP } \\
0\end{array}$ & $\begin{array}{l}\mathrm{ML} \\
\mathrm{ML}\end{array}$ & $\begin{array}{l}0.63 \\
0.36\end{array}$ & $\begin{array}{l}2.65 \\
2.60\end{array}$ \\
\hline 41 & $51541 \Upsilon$ & 081873 & Low & $\begin{array}{l}\text { Bottomland } \\
\text { flat }\end{array}$ & -- & - & -- & $\begin{array}{l}0-6 \\
6-12\end{array}$ & $\begin{array}{l}18 \\
33\end{array}$ & $\begin{array}{l}55 \\
52\end{array}$ & $\begin{array}{l}27 \\
15\end{array}$ & $\begin{array}{l}\text { S1L } \\
\text { SIL }\end{array}$ & $\begin{array}{l}87 \\
78\end{array}$ & $\begin{array}{l}40 \\
20\end{array}$ & $\begin{array}{l}24 \\
17\end{array}$ & $\begin{array}{r}16 \\
3\end{array}$ & $\begin{array}{l}\text { CL } \\
\text { ML }\end{array}$ & $\begin{array}{l}1.20 \\
0.74\end{array}$ & $\begin{array}{l}2.68 \\
2.64\end{array}$ \\
\hline 44 & \$154II & 908952 & Low & $\begin{array}{l}\text { Natural } \\
\text { levee }\end{array}$ & -- & -- & Cultivated & $\begin{array}{l}0-6 \\
6-12\end{array}$ & $\begin{array}{l}14 \\
15\end{array}$ & $\begin{array}{l}55 \\
50\end{array}$ & $\begin{array}{l}31 \\
35\end{array}$ & $\begin{array}{l}\text { SICI } \\
\text { SICL }\end{array}$ & $\begin{array}{l}90 \\
89\end{array}$ & $\begin{array}{l}56 \\
63\end{array}$ & $\begin{array}{l}27 \\
40\end{array}$ & $\begin{array}{l}29 \\
23\end{array}$ & $\begin{array}{l}\mathrm{CH} \\
\mathrm{MHA}\end{array}$ & $\begin{array}{l}1.98 \\
1.44\end{array}$ & $\begin{array}{l}2.61 \\
2.61\end{array}$ \\
\hline 46 & $5154 \mathrm{II}$ & 046963 & Low & $\begin{array}{l}\text { Natural } \\
\text { 1evee }\end{array}$ & -- & -- & Cultivated & $\begin{array}{l}0-6 \\
6-12\end{array}$ & $\begin{array}{r}10 \\
6\end{array}$ & $\begin{array}{l}67 \\
60\end{array}$ & $\begin{array}{l}23 \\
34\end{array}$ & $\begin{array}{r}\text { SiL } \\
\text { SiCL }\end{array}$ & $\begin{array}{l}94 \\
96\end{array}$ & $\begin{array}{l}44 \\
61\end{array}$ & $\begin{array}{l}25 \\
35\end{array}$ & $\begin{array}{l}19 \\
26\end{array}$ & $\begin{array}{l}\mathrm{CL} \\
\mathrm{MH}\end{array}$ & $\begin{array}{l}0.87 \\
0.78\end{array}$ & $\begin{array}{l}2.64 \\
2.66\end{array}$ \\
\hline 47 & 515411 & 052974 & High & Lower slope & - & -- & -- & $\begin{array}{l}0-6 \\
6-12\end{array}$ & $\begin{array}{l}69 \\
75\end{array}$ & $\begin{array}{l}29 \\
24\end{array}$ & $\begin{array}{l}2 \\
1\end{array}$ & $\begin{array}{l}\text { SL } \\
\text { LS }\end{array}$ & $\begin{array}{l}39 \\
32\end{array}$ & -- & -- & $\begin{array}{l}\text { NP } \\
\text { NP }\end{array}$ & $\begin{array}{l}S M \\
S M\end{array}$ & $\begin{array}{c}0.67 \\
--\end{array}$ & $\begin{array}{l}2.60 \\
2.65\end{array}$ \\
\hline 48 & $5154 \mathrm{II}$ & 069035 & Low & $\begin{array}{l}\text { Bottomland } \\
\text { flat }\end{array}$ & - & -- & Gultivated & $\begin{array}{l}0-6 \\
6-12\end{array}$ & $\begin{array}{l}15 \\
20\end{array}$ & $\begin{array}{l}84 \\
72\end{array}$ & $\begin{array}{l}1 \\
8\end{array}$ & $\begin{array}{l}\text { S1 } \\
\text { S1L }\end{array}$ & $\begin{array}{l}90 \\
85\end{array}$ & $\begin{array}{l}19 \\
17\end{array}$ & $\begin{array}{l}18 \\
16\end{array}$ & $\begin{array}{l}1 \\
1\end{array}$ & $\begin{array}{l}\text { ML } \\
\text { ML }\end{array}$ & $\begin{array}{l}0.70 \\
0.74\end{array}$ & $\begin{array}{l}2.60 \\
2.60\end{array}$ \\
\hline 49 & $5154 \mathrm{I}$ & 123124 & Low & Terrace flat & $t--$ & -- & Cultivated & $\begin{array}{l}0-6 \\
6-12\end{array}$ & $\begin{array}{l}59 \\
58\end{array}$ & $\begin{array}{l}32 \\
35\end{array}$ & $\begin{array}{l}9 \\
7\end{array}$ & $\begin{array}{l}\text { SL } \\
\text { SL }\end{array}$ & $\begin{array}{l}58 \\
58\end{array}$ & $\begin{array}{l}27 \\
21\end{array}$ & $\begin{array}{l}26 \\
--\end{array}$ & $\begin{array}{r}1 \\
\mathrm{NP}\end{array}$ & $\begin{array}{l}\text { MIL } \\
\text { ML. }\end{array}$ & $\begin{array}{c}1.10 \\
--\end{array}$ & $\begin{array}{l}2.61 \\
2.65\end{array}$ \\
\hline 50 & $5154 \mathrm{I}$ & 116087 & Low & $\begin{array}{l}\text { Natural } \\
\text { levee }\end{array}$ & -- & - & Cultivated & $\begin{array}{l}0-6 \\
6-12\end{array}$ & $\begin{array}{l}18 \\
18\end{array}$ & $\begin{array}{l}58 \\
50\end{array}$ & $\begin{array}{l}24 \\
32\end{array}$ & $\begin{array}{r}\text { S1L } \\
\text { S1CL }\end{array}$ & $\begin{array}{l}86 \\
84\end{array}$ & $\begin{array}{l}39 \\
54\end{array}$ & $\begin{array}{l}21 \\
22\end{array}$ & $\begin{array}{l}18 \\
32\end{array}$ & $\begin{array}{l}\mathrm{CL} \\
\mathrm{CH}\end{array}$ & - & $\begin{array}{l}2.70 \\
2.76\end{array}$ \\
\hline 54 & $5154 \mathrm{I}$ & 011092 & Low & $\begin{array}{l}\text { Natural } \\
\text { levee }\end{array}$ & -- & -- & Cultivated & $\begin{array}{l}0-6 \\
6-12\end{array}$ & $\begin{array}{r}18 \\
5\end{array}$ & $\begin{array}{l}50 \\
61\end{array}$ & $\begin{array}{l}32 \\
34\end{array}$ & $\begin{array}{l}\text { SICL } \\
\text { SICL }\end{array}$ & $\begin{array}{l}86 \\
96\end{array}$ & $\begin{array}{l}39 \\
49\end{array}$ & $\begin{array}{l}21 \\
23\end{array}$ & $\begin{array}{l}18 \\
26\end{array}$ & $\begin{array}{l}\text { CL } \\
\text { CL }\end{array}$ & -- & $\begin{array}{l}2.65 \\
2.69\end{array}$ \\
\hline 56 & $5154 \mathrm{I}$ & 972100 & Low & Terrace flat & t -- & -- & Cultivated & $\begin{array}{l}0-6 \\
6-12\end{array}$ & $\begin{array}{l}21 \\
23\end{array}$ & $\begin{array}{l}67 \\
77\end{array}$ & $\begin{array}{r}12 \\
0\end{array}$ & $\begin{array}{l}\text { SiL } \\
\text { SiL }\end{array}$ & $\begin{array}{l}86 \\
83\end{array}$ & $\begin{array}{l}27 \\
40\end{array}$ & $\begin{array}{l}21 \\
18\end{array}$ & $\begin{array}{r}6 \\
22\end{array}$ & $\begin{array}{l}\text { CL-ML } \\
\text { CL. }\end{array}$ & $\overline{--}$ & $\begin{array}{l}2.62 \\
2.69\end{array}$ \\
\hline
\end{tabular}




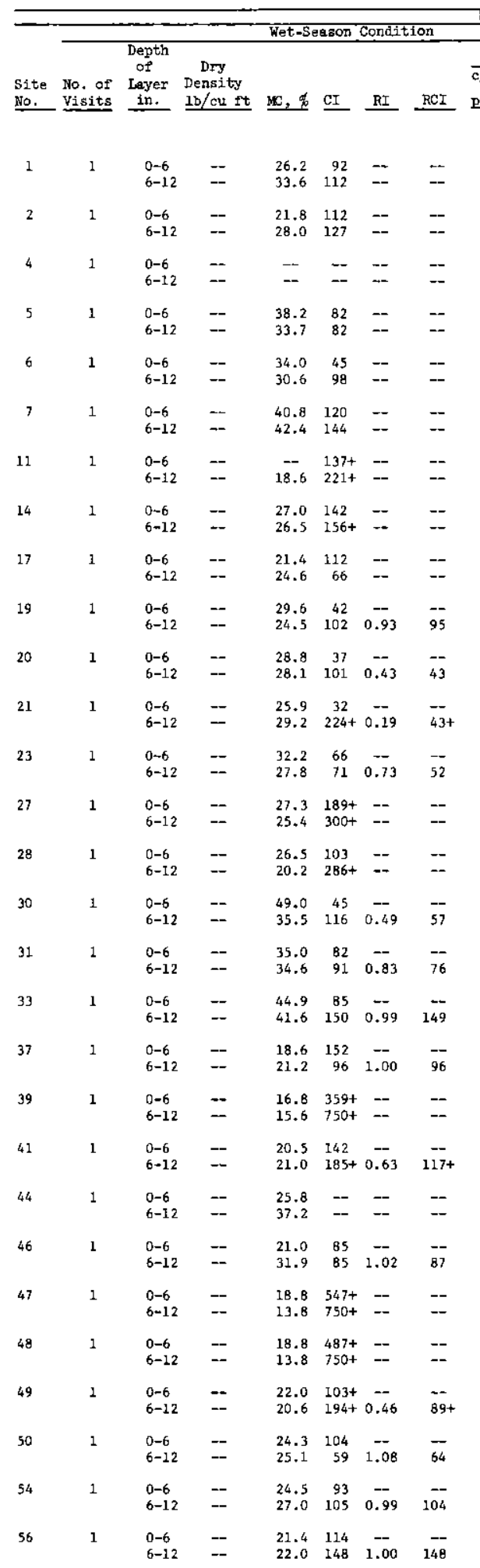

C. Sheargraph

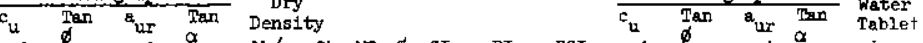

Lop Buri Area $-$ $-$

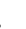

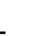
$-$

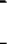

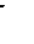
$\overline{-}$ $\overline{95}$ $-$ $4+$ $-$ $-$ $-$ 57

76

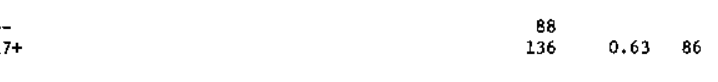

$$
-
$$$$
7
$$$$
--
$$$$
--
$$$$
-
$$$$
29+
$$$$
\begin{array}{cccc}
82 & & +3.0 \\
135+ & 0.46 & 62+ & +2.0 \\
61 & & & \\
49 & 1.08 & 53 & +2.5 \\
78 & & & \\
89 & 0.99 & 88 &
\end{array}
$$

(Continued)

\footnotetext{
** $c_{v}$, ultinate soil-to-soil cohesion; $\phi_{u 1}$, ultimate soil-to-soil angle of internal friction; a ${ }^{2}$, ultimete soil-to-rubber adhesion; $\alpha_{u r}$, ultimate soil-to-rubber angle of friction.

+ Plus (+) denotes depth of water above surfiace. 


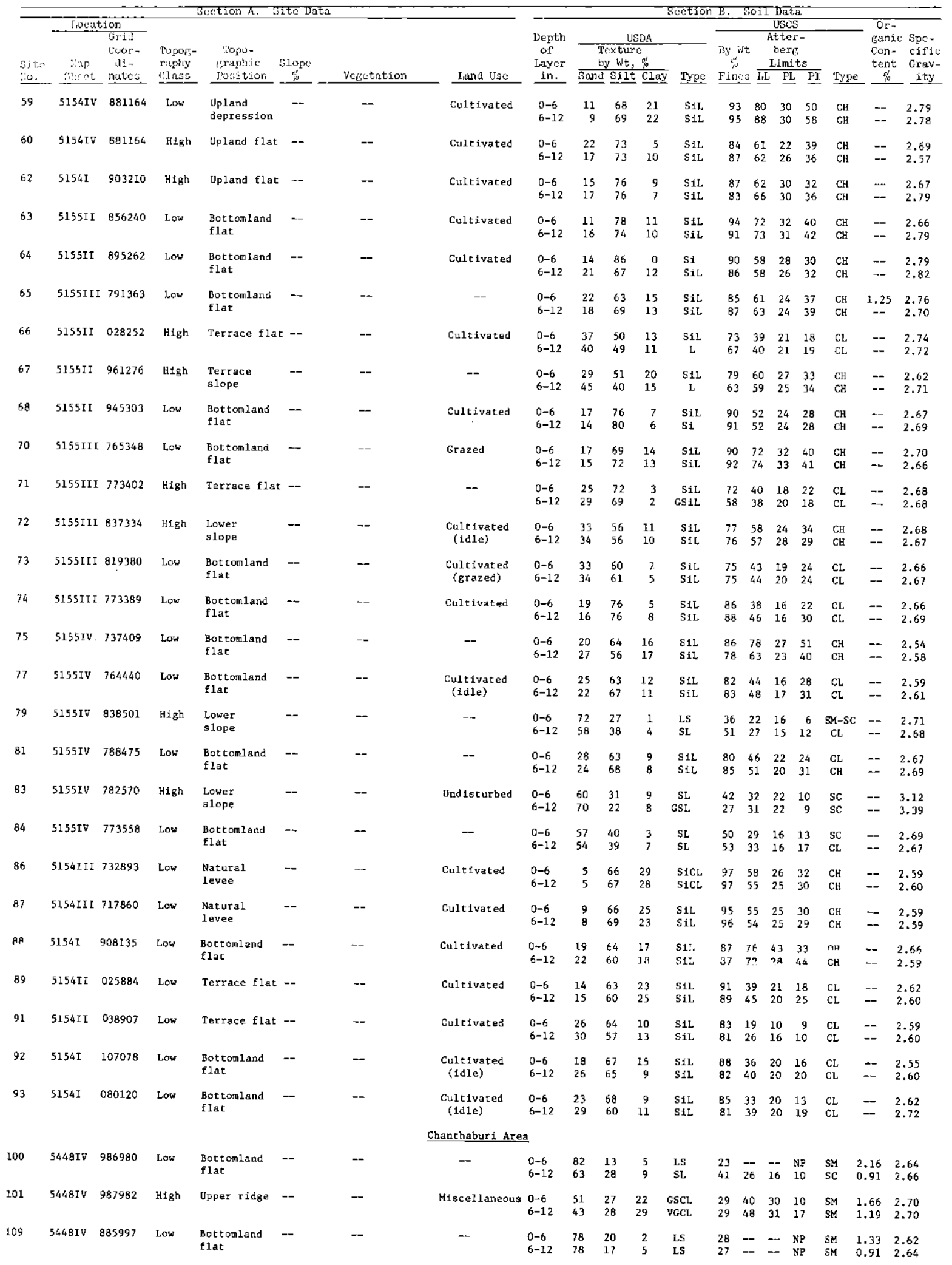




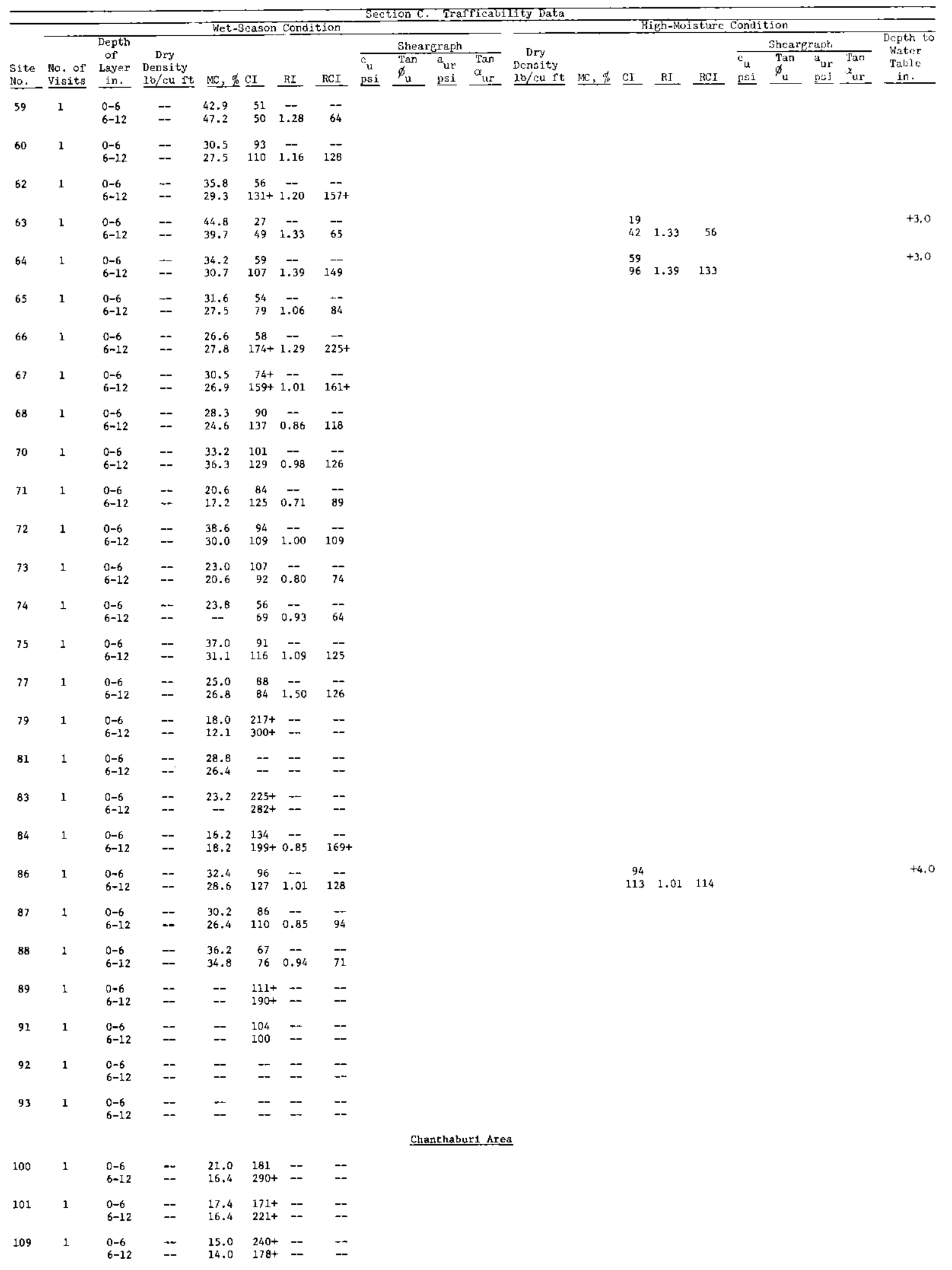




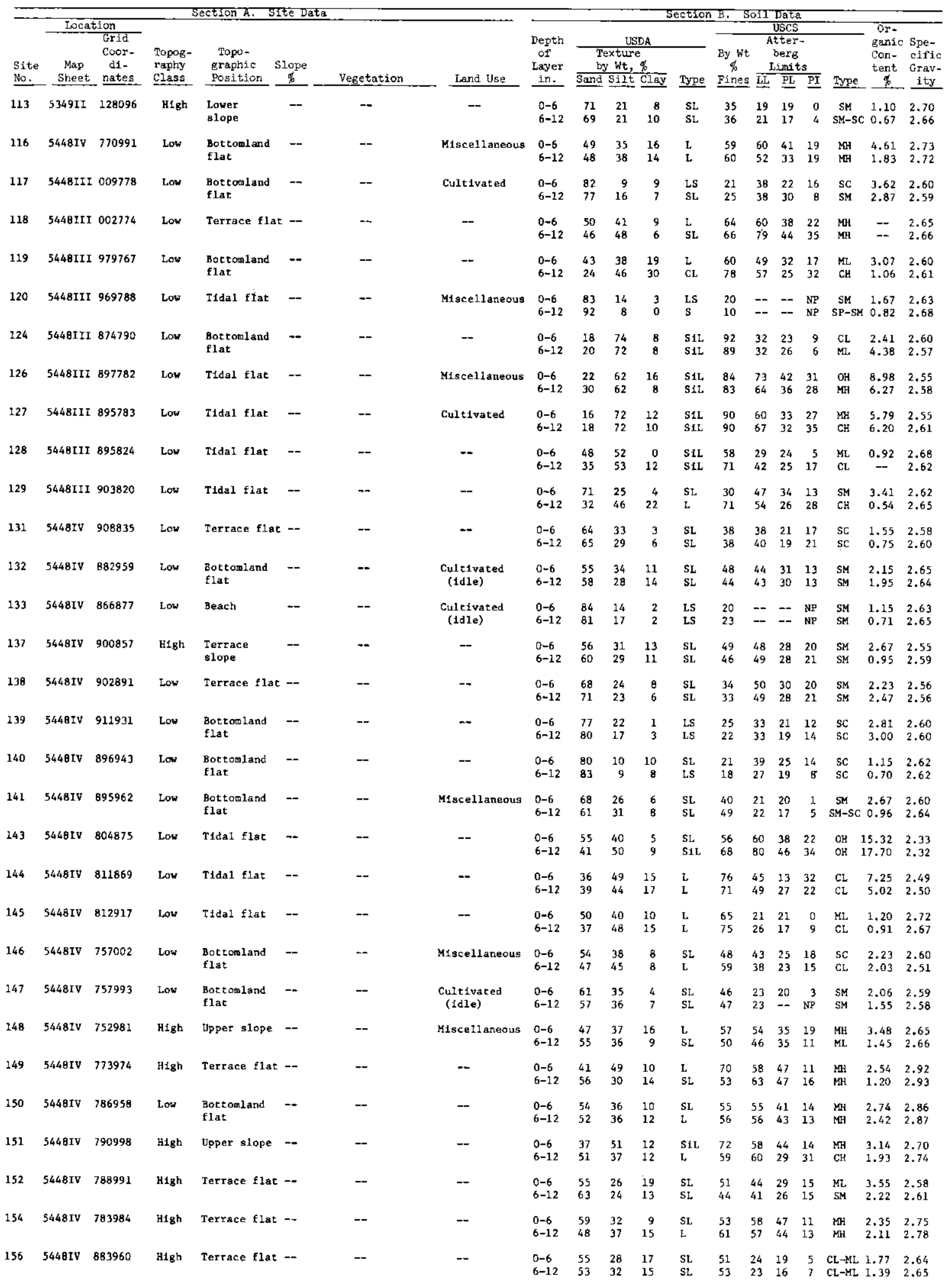


Table A5 (Continued)

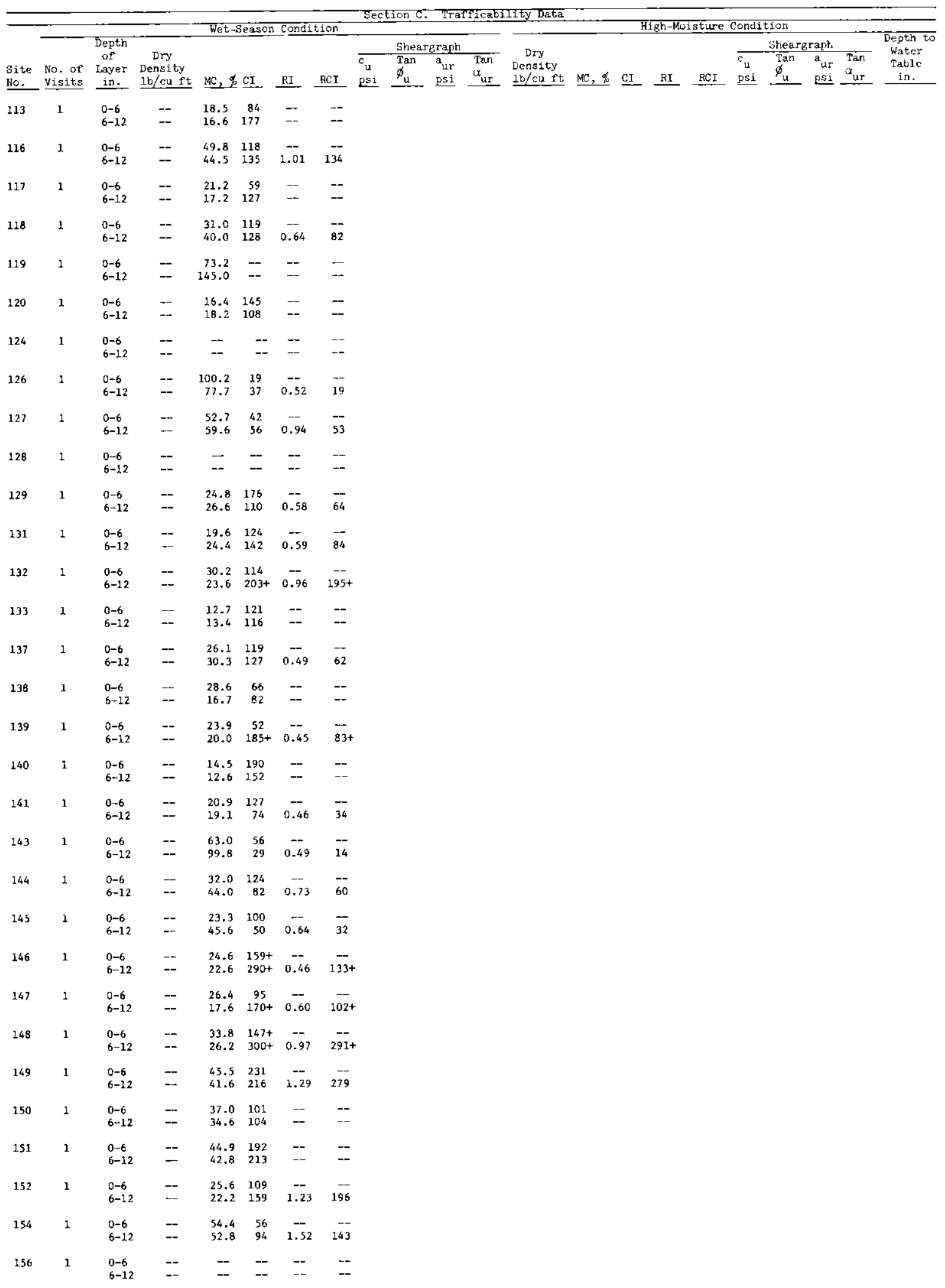




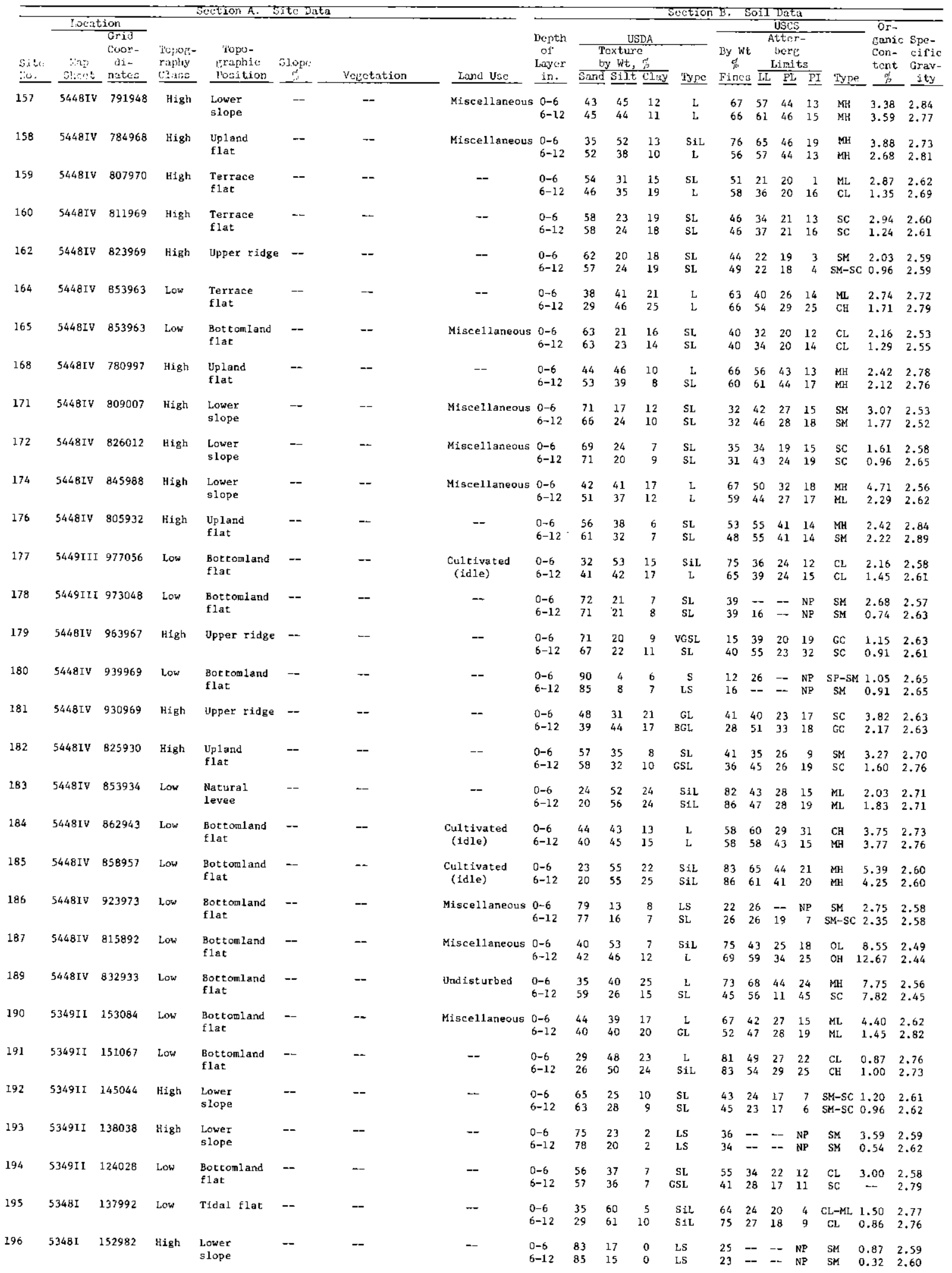




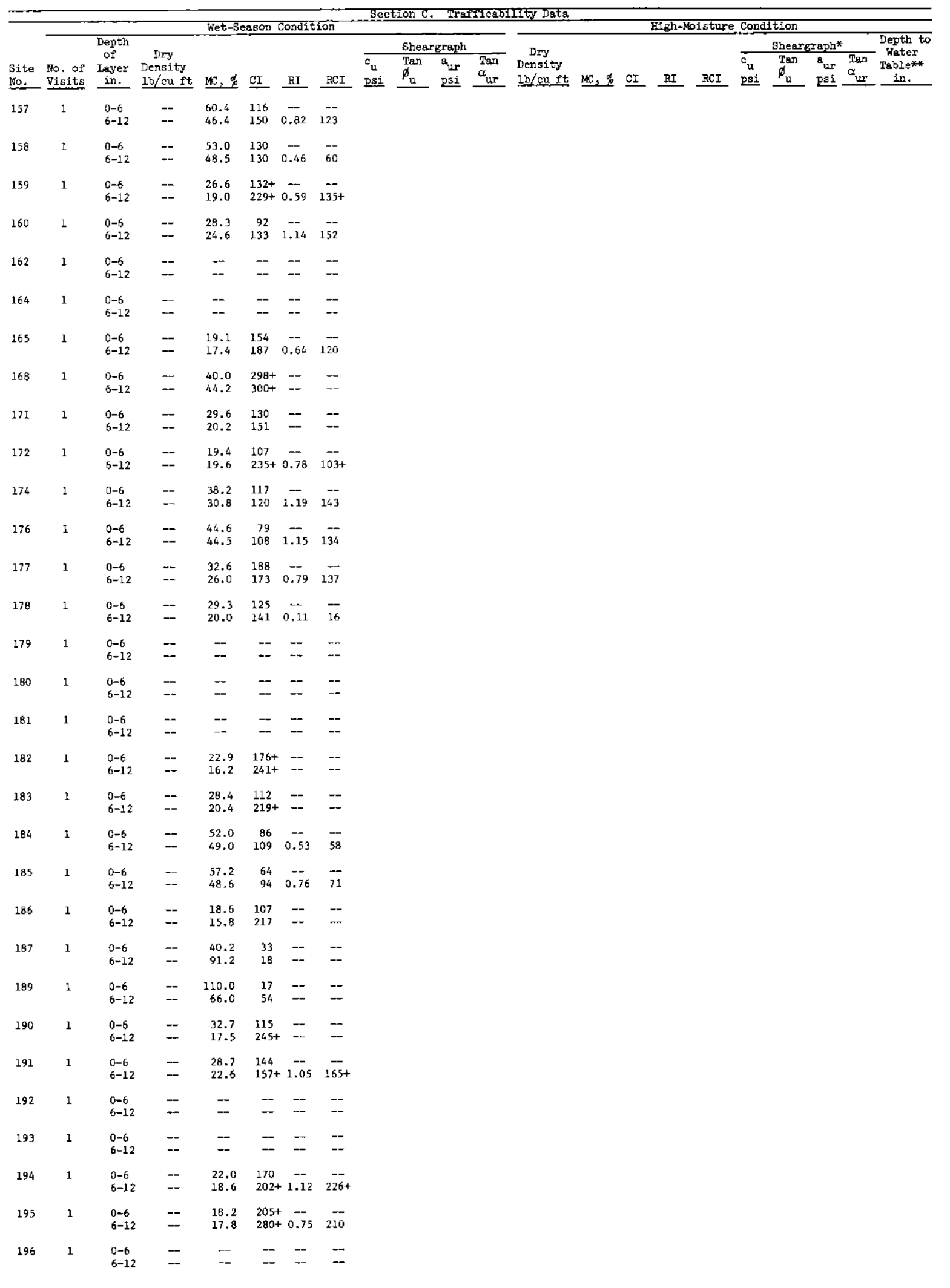


Table $A 6$

Terrain-Vehicle Tests

Summary of site, Soil, and Trafficability Data

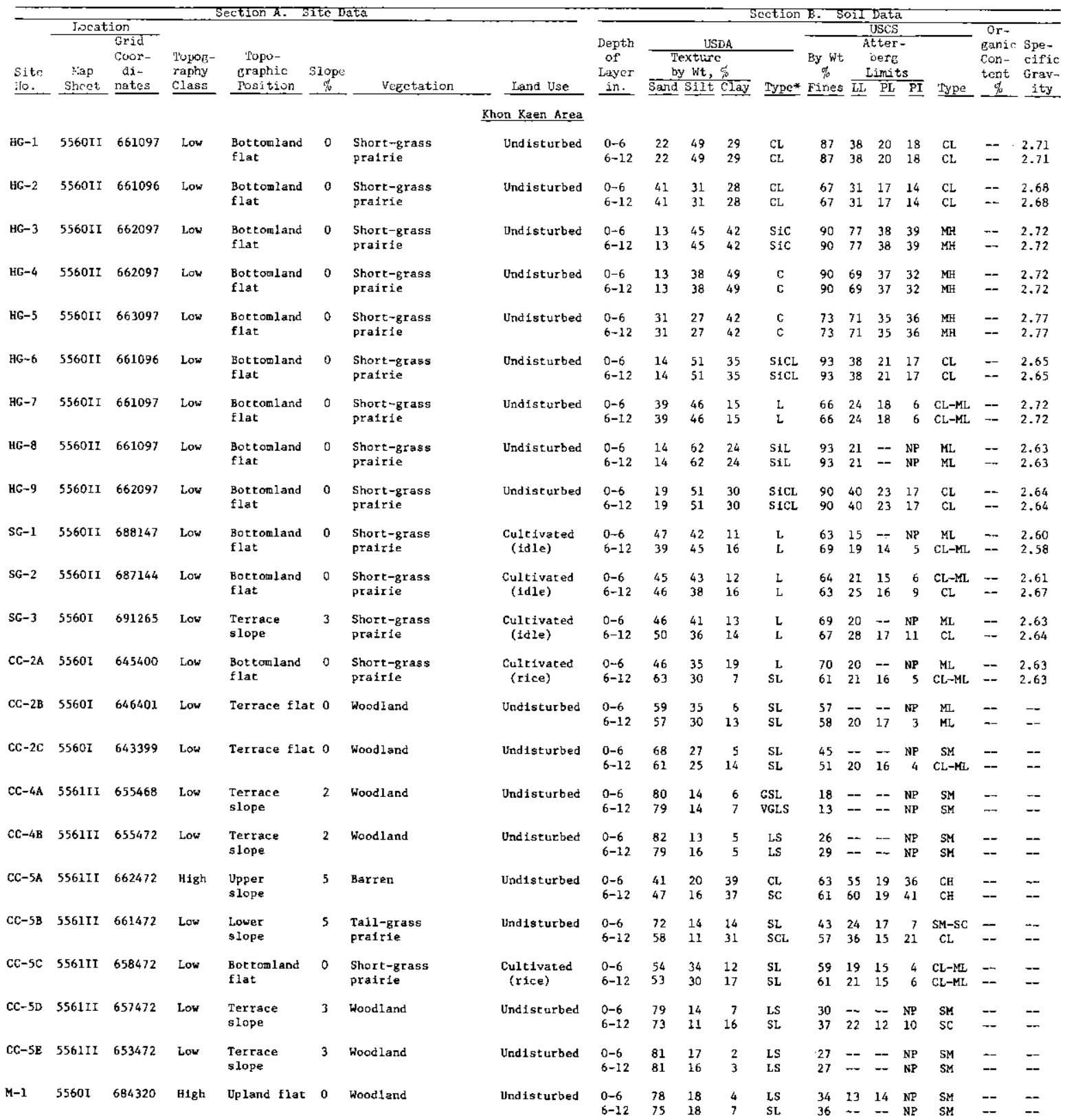




\begin{tabular}{|c|c|c|c|c|c|c|c|c|c|c|c|c|c|c|c|c|c|c|c|c|c|}
\hline \multirow{4}{*}{$\begin{array}{l}\text { Site } \\
\text { No. }\end{array}$} & \multicolumn{11}{|c|}{ Wet-Sesson Condition } & \multicolumn{10}{|c|}{ Pigh-Moisture Condition } \\
\hline & \multirow{3}{*}{$\begin{array}{l}\text { No. of } \\
\text { Visits }\end{array}$} & \multirow{3}{*}{$\begin{array}{c}\text { Depth } \\
\text { of } \\
\text { Layer } \\
\text { in. } \\
\end{array}$} & \multirow{3}{*}{$\begin{array}{c}\text { Dry } \\
\text { Density } \\
\text { lb/cu ft }\end{array}$} & \multirow{3}{*}{$\underline{M}, 2 \%$} & \multirow{3}{*}{$\mathrm{CI}$} & \multirow{3}{*}{$\underline{\mathrm{RI}}$} & \multirow{3}{*}{$\underline{\mathrm{RGI}}$} & \multirow{3}{*}{$\begin{array}{l}\overline{c_{u}} \\
\text { psi }\end{array}$} & \multicolumn{3}{|c|}{ Sheargraph ** } & \multirow{3}{*}{$\begin{array}{l}\begin{array}{c}\text { Dry } \\
\text { Density } \\
\text { lb/cu ft }\end{array} \\
\text { ea }\end{array}$} & \multirow{3}{*}{ MC, $\%$} & \multirow{3}{*}{$\underline{C I}$} & \multirow{3}{*}{$\underline{\mathrm{RI}}$} & \multirow{3}{*}{ RCI } & \multicolumn{4}{|c|}{ Sheargraph ${ }^{*}$} & \multirow{2}{*}{$\begin{array}{l}\text { Depth to } \\
\text { Water } \\
\text { Tablet } \\
\text { in. } \\
\end{array}$} \\
\hline & & & & & & & & & $\begin{array}{l}\operatorname{Tan} \\
\phi \\
\end{array}$ & $\begin{array}{l}{ }^{a} u r \\
\text { psi }\end{array}$ & $\begin{array}{r}\operatorname{Tan} \\
\alpha \\
\alpha_{u r} \\
\end{array}$ & & & & & & $\begin{array}{l}c_{u} \\
p s i\end{array}$ & $\begin{array}{l}\operatorname{Tan}_{\text {an }} \\
\phi_{u} \\
\end{array}$ & $\begin{array}{l}{ }^{\mathrm{a}} \mathrm{ur} \\
\mathrm{usi} \\
\mathrm{psi}\end{array}$ & $\begin{array}{r}\text { Tan } \\
\alpha_{\text {ur }} \\
\end{array}$ & \\
\hline & & & & & & & & & \multicolumn{3}{|c|}{ Khon Kaen Arrea } & & & & & & & & & & \\
\hline$H G-1$ & 1 & $\begin{array}{l}0-6 \\
6-12\end{array}$ & $\overline{--}$ & $\begin{array}{l}28.3 \\
28.5\end{array}$ & $\begin{array}{l}135 \\
313+\end{array}$ & $\begin{array}{l}0.72 \\
0.58\end{array}$ & $\begin{array}{c}97 \\
182+\end{array}$ & 3.0 & 0.23 & -- & - & $\overline{-}$ & $\begin{array}{l}28.3 \\
28.5\end{array}$ & $\begin{array}{l}135 \\
313+\end{array}$ & $\begin{array}{r}0.72 \\
-0.58\end{array}$ & $\begin{array}{c}97 \\
182+\end{array}$ & 3.0 & 0.23 & -- & -- & 0 \\
\hline $\mathrm{HG}-2$ & 1 & $\begin{array}{l}0-6 \\
6-12\end{array}$ & $=$ & $\begin{array}{l}26.6 \\
26.5\end{array}$ & $\begin{array}{r}71 \\
165\end{array}$ & $\begin{array}{l}0.68 \\
0.67\end{array}$ & $\begin{array}{r}48 \\
111\end{array}$ & 2.9 & 0.40 & -- & -- & -- & $\begin{array}{l}26.6 \\
26.5\end{array}$ & $\begin{array}{r}71 \\
165\end{array}$ & $\begin{array}{l}0.68 \\
0.67\end{array}$ & $\begin{array}{r}48 \\
111\end{array}$ & 2.9 & 0.40 & -- & -- & 0 \\
\hline $\mathrm{HG}-3$ & 1 & $\begin{array}{l}0-6 \\
6-12\end{array}$ & $=$ & $\begin{array}{l}29.2 \\
31.6\end{array}$ & $\begin{array}{r}86 \\
175\end{array}$ & $\begin{array}{l}0.48 \\
0.43\end{array}$ & $\begin{array}{l}41 \\
75\end{array}$ & 0.2 & 0.18 & -- & -- & -- & $\begin{array}{l}29.2 \\
31.6\end{array}$ & $\begin{array}{r}86 \\
175\end{array}$ & $\begin{array}{l}0.48 \\
0.43\end{array}$ & $\begin{array}{l}41 \\
75\end{array}$ & 0.2 & 0.18 & - & - & +1 \\
\hline $\mathrm{HG}-4$ & 1 & $\begin{array}{l}0-6 \\
6-12\end{array}$ & -- & $\begin{array}{l}26.6 \\
30.1\end{array}$ & $\begin{array}{l}139 \\
246\end{array}$ & $\begin{array}{l}0.70 \\
0.58\end{array}$ & $\begin{array}{r}97 \\
143\end{array}$ & 1.5 & 0.22 & -- & -- & -- & $\begin{array}{l}26.6 \\
30.1\end{array}$ & $\begin{array}{l}139 \\
246\end{array}$ & $\begin{array}{l}0.70 \\
0.58\end{array}$ & $\begin{array}{r}97 \\
143\end{array}$ & 1.5 & 0.22 & -- & -- & 0 \\
\hline $\mathrm{HG}-5$ & 1 & $\begin{array}{l}0-6 \\
6-12\end{array}$ & $\begin{array}{l}92.3 \\
--\end{array}$ & $\begin{array}{l}27.5 \\
31.0\end{array}$ & $\begin{array}{l}142 \\
180\end{array}$ & $\begin{array}{l}0.80 \\
0.74\end{array}$ & $\begin{array}{l}114 \\
133\end{array}$ & 1.7 & 0.40 & -- & -- & 92.3 & $\begin{array}{l}27.5 \\
31.0\end{array}$ & $\begin{array}{l}142 \\
180\end{array}$ & $\begin{array}{l}0.80 \\
0.74\end{array}$ & $\begin{array}{l}114 \\
133\end{array}$ & 1.7 & 0.40 & -- & -- & 0 \\
\hline $\mathrm{HG}-6$ & 2 & $\begin{array}{l}0-6 \\
5-12\end{array}$ & $\begin{array}{l}99.3 \\
98.4\end{array}$ & $\begin{array}{l}24.0 \\
25.5\end{array}$ & $\begin{array}{r}44 \\
115\end{array}$ & $\begin{array}{l}0.70 \\
0.66\end{array}$ & $\begin{array}{l}31 \\
76\end{array}$ & 0.0 & 0.16 & -- & -- & $\begin{array}{l}99.3 \\
98.4\end{array}$ & $\begin{array}{l}24.0 \\
25.5\end{array}$ & $\begin{array}{r}44 \\
115\end{array}$ & $\begin{array}{l}0.70 \\
0.66\end{array}$ & $\begin{array}{l}31 \\
76\end{array}$ & 0.0 & 0.16 & -- & . & 0 \\
\hline $\mathrm{HG}-7$ & 1 & $\begin{array}{l}0-6 \\
6-12\end{array}$ & $\stackrel{104.1}{--}$ & $\begin{array}{l}21.7 \\
19.9\end{array}$ & $\begin{array}{r}73 \\
207\end{array}$ & $\begin{array}{l}0.62 \\
0.45\end{array}$ & $\begin{array}{l}45 \\
93\end{array}$ & 1.2 & 0.18 & -- & -- & 104.1 & $\begin{array}{l}21.7 \\
19.9\end{array}$ & $\begin{array}{r}73 \\
207\end{array}$ & $\begin{array}{l}0.62 \\
0.45\end{array}$ & $\begin{array}{l}45 \\
93\end{array}$ & 1.2 & 0.18 & -- & -- & 0 \\
\hline HG-8 & 1 & $\begin{array}{l}0-6 \\
6-12\end{array}$ & $\stackrel{100.5}{--}$ & $\begin{array}{l}21.5 \\
22.4\end{array}$ & $\begin{array}{l}111 \\
299\end{array}$ & $\begin{array}{l}0.61 \\
0.77\end{array}$ & $\begin{array}{r}68 \\
230\end{array}$ & 0.0 & 0.34 & -- & -- & 100.5 & $\begin{array}{l}21.5 \\
22.4\end{array}$ & $\begin{array}{l}111 \\
299\end{array}$ & $\begin{array}{l}0.61 \\
0.77\end{array}$ & $\begin{array}{r}68 \\
230\end{array}$ & 0.0 & 0.34 & -- & -- & 0 \\
\hline $4 G-9$ & 1 & $\begin{array}{l}0-6 \\
6-12\end{array}$ & $\begin{array}{l}97.2 \\
--\end{array}$ & $\begin{array}{l}23.4 \\
28.0\end{array}$ & $\begin{array}{r}45 \\
138\end{array}$ & $\begin{array}{l}0.60 \\
0.61\end{array}$ & $\begin{array}{l}27 \\
84\end{array}$ & 0.0 & 0.18 & -- & -- & 97.2 & $\begin{array}{l}23.4 \\
28.0\end{array}$ & $\begin{array}{r}45 \\
138\end{array}$ & $\begin{array}{l}0.60 \\
0.61\end{array}$ & $\begin{array}{l}27 \\
84\end{array}$ & 0.0 & 0.18 & - & -- & 0 \\
\hline $5 G-1$ & 3 & $\begin{array}{l}0-6 \\
6-12\end{array}$ & $\begin{array}{l}102.0 \\
109.6\end{array}$ & $\begin{array}{l}17.3 \\
16.6\end{array}$ & $\begin{array}{r}96 \\
182\end{array}$ & $\begin{array}{l}0.43 \\
0.46\end{array}$ & $\begin{array}{l}36 \\
74\end{array}$ & 1.3 & 0.37 & 0.3 & 0.40 & $\begin{array}{l}106.9 \\
107.0\end{array}$ & $\begin{array}{l}17.0 \\
18.4\end{array}$ & $\begin{array}{r}72 \\
161\end{array}$ & $\begin{array}{l}0.33 \\
0.38\end{array}$ & $\begin{array}{l}24 \\
61\end{array}$ & 0.0 & 0.27 & 0.0 & 0.27 & +2 \\
\hline SG-2 & 1 & $\begin{array}{l}0-6 \\
6-12\end{array}$ & $\stackrel{103.6}{--}$ & 17.1 & $\begin{array}{r}85 \\
174\end{array}$ & $\stackrel{0.51}{--}$ & 43 & 2.2 & 0.55 & 0.0 & 0.47 & 103.6 & 17.1 & $\begin{array}{r}85 \\
174\end{array}$ & $\begin{array}{c}0.51 \\
--\end{array}$ & 43 & 2.2 & 0.55 & 0.0 & 0.47 & +2 \\
\hline$S G-3$ & 2 & $\begin{array}{l}0-6 \\
6-12\end{array}$ & $\begin{array}{r}102.6 \\
95.1\end{array}$ & $\begin{array}{l}20.7 \\
25.2\end{array}$ & $\begin{array}{l}111 \\
139\end{array}$ & $\begin{array}{l}0.68 \\
0.72\end{array}$ & $\begin{array}{l}76 \\
98\end{array}$ & 2.0 & 0.33 & 0.6 & 0.30 & $\begin{array}{c}103.4 \\
--\end{array}$ & $\begin{array}{l}20.4 \\
24.1\end{array}$ & $\begin{array}{l}104 \\
142\end{array}$ & $\begin{array}{l}0.56 \\
0.62\end{array}$ & $\begin{array}{l}58 \\
88\end{array}$ & 1.2 & 0.30 & 0.3 & 0.27 & +3 \\
\hline$C C-2 \mathrm{~A}$ & 2 & $\begin{array}{l}0-6 \\
6-12\end{array}$ & $\begin{array}{r}99.6 \\
107.8\end{array}$ & $\begin{array}{l}21.3 \\
17.9\end{array}$ & $\begin{array}{r}79 \\
133\end{array}$ & $\begin{array}{l}0.47 \\
0.33\end{array}$ & $\begin{array}{l}36 \\
46\end{array}$ & 0.5 & 0.32 & 0.0 & 0.34 & $\begin{array}{l}108.0 \\
106.4\end{array}$ & $\begin{array}{l}18.2 \\
18.7\end{array}$ & $\begin{array}{l}72 \\
86\end{array}$ & $\begin{array}{l}0.63 \\
0.26\end{array}$ & $\begin{array}{l}45 \\
22\end{array}$ & 0.5 & 0.32 & 0.0 & 0.34 & +6 \\
\hline$C C-2 B$ & 1 & $\begin{array}{l}0-6 \\
6-12\end{array}$ & $\begin{array}{l}110.7 \\
104.1\end{array}$ & $\begin{array}{l}24.0 \\
13.3\end{array}$ & $\begin{array}{l}225 \\
237\end{array}$ & -- & -- & 2.7 & 0.36 & 1.0 & 0.32 & & & & & & & & & & \\
\hline $\mathrm{CC}-2 \mathrm{C}$ & 1 & $\begin{array}{l}0-6 \\
6-12\end{array}$ & $\begin{array}{l}101.6 \\
107.6\end{array}$ & $\begin{array}{l}11.1 \\
13.0\end{array}$ & $\begin{array}{l}214 \\
239\end{array}$ & $=$ & -- & 1.6 & 0.55 & 0.0 & 0.51 & & & & & & & & & & \\
\hline $\mathrm{CC}-4 \mathrm{~A}$ & 1 & $\begin{array}{l}0-6 \\
6-12\end{array}$ & -- & -- & $\begin{array}{l}399+ \\
699+\end{array}$ & -- & -- & 0.2 & 0.38 & 0.2 & 0.42 & & & & & & & & & & \\
\hline$C \mathrm{C}-4 \mathrm{~B}$ & 1 & $\begin{array}{l}0-6 \\
6-12\end{array}$ & $=$ & 9.5 & $\begin{array}{l}169 \\
130\end{array}$ & -- & $=$ & 2.3 & 0.36 & 1.5 & 0.25 & & & & & & & & & & \\
\hline CC-5A & 1 & $\begin{array}{l}0-6 \\
6-12\end{array}$ & $=$ & $\begin{array}{l}20.4 \\
20.1\end{array}$ & $\begin{array}{l}616+ \\
750+\end{array}$ & -- & -- & - & - & 0.0 & 0.36 & & & & & & & & & & \\
\hline$C C-5 B$ & 1 & $\begin{array}{l}0-6 \\
6-12\end{array}$ & $\begin{array}{r}98.2 \\
105.3\end{array}$ & $\begin{array}{l}10.5 \\
17.1\end{array}$ & $\begin{array}{l}365 \\
296\end{array}$ & -- & -- & 2.0 & 0.47 & 0.0 & 0.47 & & & & & & & & & & \\
\hline $\mathrm{CC}-\mathrm{SC}$ & 1 & $\begin{array}{l}0-6 \\
6-12\end{array}$ & $\begin{array}{r}109.2 \\
99.1\end{array}$ & $\begin{array}{l}17.2 \\
20.9\end{array}$ & $\begin{array}{r}98 \\
136\end{array}$ & $\begin{array}{l}0.32 \\
0.43\end{array}$ & $\begin{array}{l}31 \\
58\end{array}$ & 2.8 & 0.34 & 1.2 & 0.23 & $\begin{array}{r}109.2 \\
99.1\end{array}$ & $\begin{array}{l}17.2 \\
20.9\end{array}$ & $\begin{array}{r}98 \\
136\end{array}$ & $\begin{array}{l}0.32 \\
0.43\end{array}$ & $\begin{array}{l}31 \\
58\end{array}$ & 2.8 & 0.34 & 1.2 & 0.23 & +3 \\
\hline$C C-5 D$ & 1 & $\begin{array}{l}0-6 \\
6-12\end{array}$ & -- & $\begin{array}{l}12.2 \\
14.9\end{array}$ & $\begin{array}{l}145 \\
188\end{array}$ & $=$ & $=$ & 2.0 & 0.45 & 0.6 & 0.38 & & & & & & & & & & \\
\hline$C C-5 E$ & 1 & $\begin{array}{l}0-6 \\
6-12\end{array}$ & $\begin{array}{r}94.8 \\
100.5\end{array}$ & $\begin{array}{l}23.2 \\
19.3\end{array}$ & $\begin{array}{l}33 \\
46\end{array}$ & $\begin{array}{l}0.36 \\
0.75\end{array}$ & $\begin{array}{l}12 \\
35\end{array}$ & 2.4 & 0.40 & 0.6 & 0.38 & $\begin{array}{r}94.8 \\
100.5\end{array}$ & $\begin{array}{l}23.2 \\
19.3\end{array}$ & $\begin{array}{l}33 \\
46\end{array}$ & $\begin{array}{l}0.36 \\
0.75\end{array}$ & $\begin{array}{l}12 \\
35\end{array}$ & 2.4 & 0.40 & 0.6 & 0.38 & 0 \\
\hline$M-1$ & 2 & $\begin{array}{l}0-6 \\
6-12\end{array}$ & $\begin{array}{r}102.0 \\
99.8\end{array}$ & $\begin{array}{l}12.5 \\
12.7\end{array}$ & $\begin{array}{l}154 \\
129\end{array}$ & $=-$ & -- & 2.0 & 0.42 & 0.6 & 0.40 & & & & & & & & & & \\
\hline
\end{tabular}

** $c_{u}$, wltimate soil-to-soil cohesion; $\phi_{u}$, ultimate soil-to-soil angle of intemal friction; a $u$, ultimate soil-to-mubber adhesion;

$\alpha_{u r}$, ultimate soil-to-rubber ansle of friction.

+ Plus (+) denotes depth of water above surface. 

Secretary of Defense, ATTN: ARPA/AGILE, The Pentagon, Washington, D. C.

Commanding GeneraI, U. S. Army Materiel Command, ATTN: AMCRD-RV-E, 2 Washington, D. C.

Dr. Daniel C. Drucker, Physical Sciences Council, Brown University, Providence, R. I.

Dr. Emil H. Jebe, Operations Research Dept., Institute of Science and Technology, University of Michigan, Box 618, Ann Arbor, Mich.

Commanding General, U. S. Army Tank-Automotive Center, ATTN: SMOTA-RCI (Mr. R. A. Iiston), Warren, Mich.

Dr. Ralph E. Fadum, Dean, School of Engineering, North Carolina State College of the University of North Carolina, Raleigh, N. C.

Prof. Robert Horonjeff, 3643 Brook st., Lafayette, Calif.

Director, Joint Research and Test Activity,

APO San Francisco 96243

Director, OSD/ARPA R\&D Field Unit, APO San Francisco 96243

Director, OSD/ARPA R\&D Field Unit, APO San Francisco 96346

Commander, U. S. MiIitary Assistance Command, APO San Francisco 96346

Dr. Clark N. Crain, Director, Project DUTY, Dept. of Geography, University of Denver, Denver, Colo.

CINCPAC, Camp H. M. Smith, Oahu, Hawaii

Mr. A. C. Orvedal, Chief, World Soil Geography Unit, Soil Conservation Service, USDA, Hyattsville, Ma.

Commanding General, U. S. Army Natick Laboratories, ATTN: AMXRE-ED (Dr. L. W. Trueblood), Natick, Mass.

Chief, Source Material Unit, Branch of Military Geology,

U. S. Geological Survey, Washington, D. C. 
Director, OSD/ARPA Regional Field Office (Latin America)

P. O. Drawer 942, Ft. Clayton, Canal Zone

Director, OSD/ARPA Regional Field Office, APO New York 09694

Director, U. S. Army Engineer Geodesy Intelligence \& Mapping 1 Research \& Development Agency, ATTN: ENGGM-IN

(Mr. J. E. Gillis, Jr.), Fort Belvoir, Va.

Wilson, Nuttall, Raimond, Engineers, Inc.,

ATTN: Mr. C. J. Nuttall, Jr., Chestertown, Ma.

Commanding Officer, U. S. Army Cold Regions Research and Engineering Laboratory, ATTN: AMXCR-PI (Mr. R. E. Frost), ATTN: Library, P. O. Box 282, Hanover, N. H. 1

President, U. S. Army Armor Board, Fort Knox, Ky. 1

President, U. S. Army Air Defense Board, Fort Bliss, Tex. 1

President, U. S. Army Infantry Board, Fort Benning, Ga. 1

President, U. S. Army Infantry Board, ATTN: Tactical Section, TIS, 1 Fort Benning, Ga.

President, U. S. Army Artillery Board, Fort Sill, Okla. 1

President, U. S. Army Airborne, Electronic-Special Warfare Board I Fort Bragg, N. C.

President, U. S. Army Aviation Test Board, Fort Rucker, Ala.

Director, U. S. Army Development \& Proof Services

ATIN: Technical Library

ATIN: Automotive Engineering Laboratory (STEAP-DS-LU)

ATTV: Automotive Division (STEAP-DS-TU)

Aberdeen Proving Ground, Md.

Chief of Engineers, Department of the Army,

ATTN: ENGSA

ATTTN: ENGTTE-E

ATTN: ENTGTE-M

ATTN: ENGTE-S

Washington, D. C.

(1) 1 1 1 . 1

Chief of Research and Development, Headquarters, Dept. of the Army, AnTN: Director of Army Technical Information Washington, D. C. 
Commanding General, U. S. Army Weapons Command, ATIN: AMSWE-RDR, Rock Island, Ill.

Commanding General, U. S. Army Forces Southern Command, ATHIN: Engineer, Fort Amador, Canal Zone

Director, Institute for Defense Analysis, 400 Army-Navy Drive, Arlington, Va.

U. S. Army Attaché, American Embassy, U. S. Navy 100, Box 36, FPO, New York

Commanding Officer, U. S. Army Engineer Research \& Development Laboratories, ATTN: Technical Documents Center, Fort Belvoir, Va.

Commandant, U. S. Army Engineer School, ATTN: Library, Fort Belvoir, Va.

Commander, U. S. Army Combat Development Command ENGINEER AGENCY, I ATrT: CAGEN-SC, Fort Belvoir, Va.

Commanding General, U. S. Continental Army Command, Engineering I Division, DCSLOG, ATTI: ATLOG-E-MB, Fort Monroe, Va.

Commanding General, U. S. Continental Army Command, ATTN: Combat I Development (ATSND), Fort Monroe, Va.

Assistant Chief of Staff for Force Development, Department of the Army, ATrN: FOR DS SSS, The Pentagon, washington, D. C.

Commanding officer \& Director, U. S. Naval Civil Engineering Laboratory, Port Hueneme, Calif.

Commanding officer, Office of Naval Research, ATrN: Naval Applications Group, Surface \& Amphibious Programs (Code 463), Washington, D. C.

Commanding Officer, U. S. Naval Photographic Interpretation Center, 1 ATTN: Iibrary, 4301 Suitland Road, Washington, D. C.

Commanding Officer, Office of Naval Research, ATrN: Earth Sciences Division (Code 410), Washington, D. C.

Commandant, U. S. Marine Corps, ATrN: AO4E, Washington, D. C.

Chief, Bureau of Yards \& Docks, ATIM: Code 70, Department of the Navy, Washington, D. C. 
Commanding Officer, PHIBCB one, U. S. Naval Amphibious Base, Coronado, San Diego, Calif.

Commanding Officer, $\mathrm{PH} \mathrm{BCB}$ T'wo, U. S. Naval Amphibious Base, Iittle Creek, Norfolk, Va.

Director, Naval Warfare Research Center, Stanford Research Institute, Menlo Park, Calif.

Commandant, U. S. Army Signal School, ATTN: Library, Fort Monmouth, N. J.

Air University Library, Maxwell AFB, Ala.

Defense Documentation Center, ATIN: Mr. Myer Kahn, Cameron Station, 20 Alexandria, Va.

New York University, ATriN: Engineering Library, University Heights, Bronx, N. Y.

Davidson Laboratory, Stevens Institute of Technology, 711 Hudson Street, Hoboken, N. J.

Commandant, U. S. Military Academy, ATrN: Library, West Point, N. Y.

Research Analysis Corporation, ATTN: Iibrary, McLean, Va. 22101

Commandant, Command \& General Staff College, ATrN: Archives, $I$ Fort Leavenworth, Kans.

Commandant, Armed Forces Staff College, ATrN: Library, Norfolk, $\mathrm{Va}$.

Commanding Officer, U. S. Army Combat Developments Command Experimentation Center, Fort Ord, Calif.

Commanding General, U. S. Army Mobility Command, ATTN: AMSMO-R, Warren, Mich.

Massachusetts Institute of Technology, ATTN: Soil Engineering Library, Cambridge, Mass.

Library of Congress, Documents Expediting Project, Washington, D. C. 
National Tillage Machinery Laboratory, U. S. Dept. of Agriculture,

P. O. Box 792, Auburn, Ala.

Commanding General, U. S. Army Electronic Proving Ground, ATIN: Iibrary, Fort Huachuca, Ariz.

Director, Project Michigan, Willow Run Laboratories, Ypsilanti, Mich.

Prof. L. C. Stuart, University of Michigan, Ann Arbor, Mich.

Engineering Societies Library, 345 E. 47 th st., New York, N. Y. 1

University of Arkansas, College of Engineering, Fayetteville, Ark. I

Library, Division of Public Documents, U. S. Government Printing 1 Office, Washington, D. C.

Commanding Officer, Picatinny Arsenal, ATTN: Ammunition Research Laboratory, Engr Res Section, Dover, N. J.

Commanding Officer, U. S. Army Rock Island Arsenal, Rock Island, Ill.

Commander, U. S. Strike Command, ATTN: J4-E, MacDill AFB, Fla.

Headquarters, USAF, ATTM: Deputy Chief of Staff, Research and Development (AFRDC) I ATTN: Deputy Chief of Staff, Director of Civil Engineering (AFOCE)

ATTN: Director of Science and Technology (AFRST) Washington, D. C.

Commander, Air Proving Ground Center, ATTN: PGBPS-12, Eglin AFB, Fla.

Commander, Systems Engineering Group, Deputy for Systems Engineering, Directorate of Technical Publications and Specifications (SEPRR), Wright-Patterson AFB, Ohio

Director, Terrestrial Sciences Laboratory (CRJT), Air Force Cambridge Research Iaboratories, L. G. Hanscom Field, Bedford, Mass. 
Commanding officer, U. S. Army Aviation Materiel Laboratories, ATCN: OSMFE-ES (Mr. H. P. Simon), Fort Eustis, Va.

Commanding Officer, Defense Intelligence Agency, ATTN: DIAAP-1E Washington, D. C.

Commanding Officer, U. S. Army Tropic Test Center, Fort Clayton, Canal Zone

Commanding officer, U. S. Army Limited War Iaboratory, ATTN: Development Engineering Division, Aberdeen Proving Ground, Md.

Commanding Officer, Special Warfare Agency, Fort Bragg, N. C.

Air Force Office of Scientific Research, Building T-D, Washington, D. C.

Battelle Memorial Institute, ATIN: RACIC, 505 King Ave., Columbus, Ohio

Battelle Memorial Institute, ATTN: RACIC, 1755 Massachusetts Ave., N. W., Washington, D. C.

GM Defense Research Laboratories, General Motors Corporation Box T, Santa Barbara, Calif. 93101 
Unclassified

Security Classification

\section{DOCUMENT CONTROL DATA - R\&D}

(Security clesaiflcation of title, body of abstract and indexing annotation muat be entered when the ovesall seport is clessified) 1. ORIGINATIN G ACTIVITY (Comporate author) Ta. REPORT SECURITY CLASSIFICATION U. S. Army Engineer Waterways Experiment Station Vicksburg, Mississippi

3. REPORT TITLE

TRAFFICABILITY CLASSIFICATION OF THAILAND SOILS

\section{Final report}

4. OESCRIPTIVE NOTES (Type of report and inclusive datos)

5. AUTHOR(S) (Last namo, first name, inttial)

Meyer, M. P.

\begin{tabular}{|c|c|c|}
\hline $\begin{array}{l}\text { 6. REPORT DATE } \\
\text { January } 1967\end{array}$ & $\begin{array}{c}\text { 7a. TOTAL NO. OF PAGES } \\
137\end{array}$ & $\begin{array}{c}7 \mathrm{~b} . \text { NO. OF REFS } \\
15\end{array}$ \\
\hline $\begin{array}{l}\text { BA. CONTRACT OR GRANT NO. } \\
\text { b. PROJECT NO. I-V-O-21701-A-046 }\end{array}$ & $\begin{array}{l}\text { 90. ORIGINATOR'S REPORT } \\
\text { Technical Repor }\end{array}$ & OER(S) \\
\hline $\begin{array}{l}\text { c. Task No. 1-V-O-21701-A-046-O2 } \\
\text { d. }\end{array}$ & $\begin{array}{l}\text { gb. OYHER REPORT NO(S) } \\
\text { this report) }\end{array}$ & othor numbers that may be assigned \\
\hline
\end{tabular}

11. SUPPL EMENTARY NOTES

12. SPONSORING MILITARY ACTIVITY

U. S. Army Materiel Command

13. ABSTRACT

Pertinent soil trafficability data were collected during the wet season at 846 sites in Thailand. The soils were identified according to the Unified Soil Classification System and the U. S. Department of Agriculture textural classification system. Two general topographic positions (high topography and low topography) and two general levels of wetness were considered. A scheme for classifying soils according to their trafficability was developed. The scheme lists the soil types in order of decreasing trafficability under each of three topography-wetness level categories and shows the probability of successful. passage on each soil for vehicles of known soil strength requirements. The scheme permits the estimation of the probability of a successful operation for given soil type, topography, and wetness-level conditions. If a choice of several routes and vehicles is available, the determination of the vehicles with the best chances of success over a given route or of the best route for given vehicles can be made. 
Unclassif'ied

Security Classification

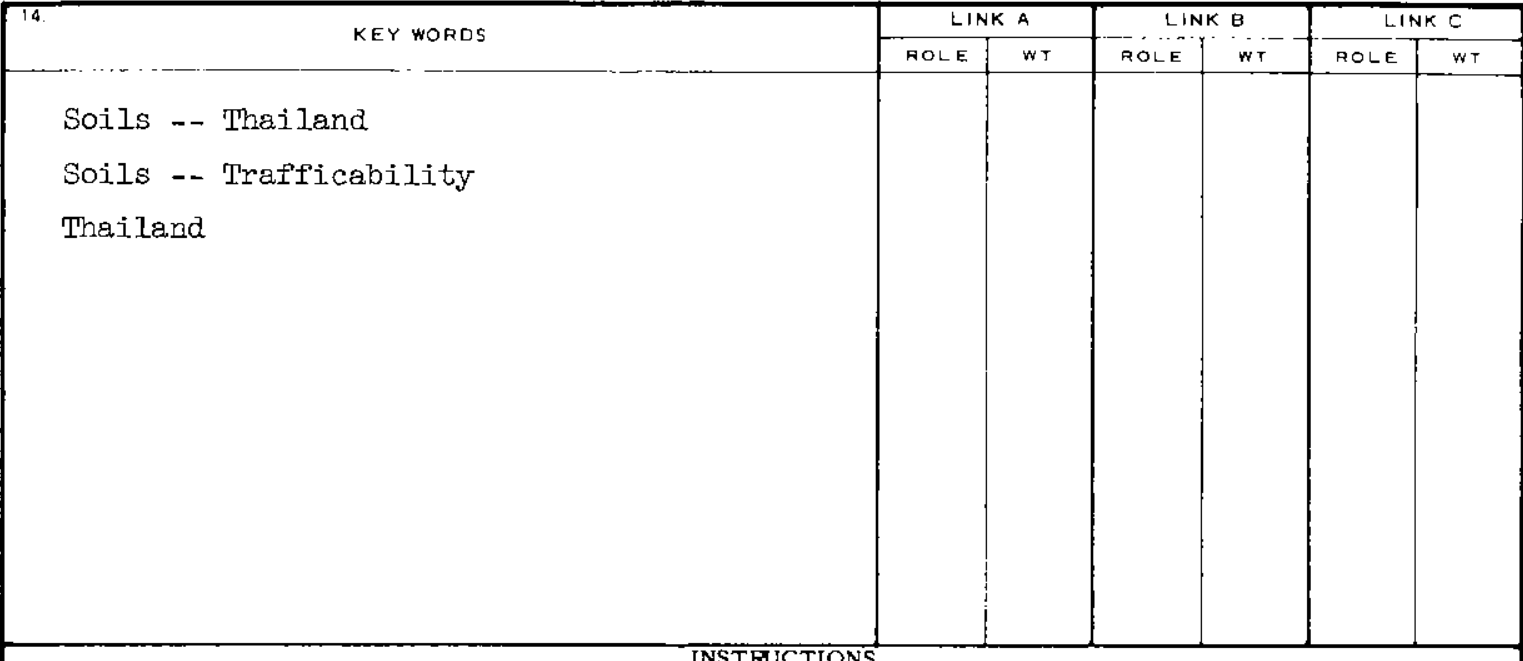

INSTRUCTIONS

1. ORIGINATING ACTIVITY: Enter the name and address of the contractor, subcontractor, grantee, Department of Defense activity or other organization (corporate author) issuing the report.

2a. REPORT SECURTY CLASSIFICATION: Enter the overall security classification of the report. Indicate whether "Restricted Data" is included Marking is to be in accordance with appropriate security regulations.

2b. GROUP: Automatic downgrading is specified in DoD Directive 5200.10 and Armed Forces Industrial Manual. Enter the group number. Also, when applicable, show that optional markings have been us ed for Group 3 and Group 4 as authorized.

3. REPORT TITLE: Enter the complete report title in al: capital letters. Titles in all cases should be unclessified. If a meaningful title cannot be selected without classification, show title classification in all capitals in parenthesis immediately following the title.

4. DESCRIP'TIVE NO'TES: If appropriate, enter the type of report, e.g., interim, progress, summary, annual, or final. Give the inclusive dates when a specific reporting period is covered.

5. AUTHOR(S): Enter the name(s) of author(s) as shown on or in the report. Enter last name, first name, middle initial. If military, show rank and branch of service. The name of the principal author is an absolute minimum requirement.

6. REPORT DATE: Enter the date of the report as day, month, year; or month, year. If more than one date appears on the report, use date of publication.

7a. TOTAL NUMBER OF PAGES: The total page count should follow normal pagination procedures, i. e., enter the number of pages containing information

7b. NUMBER OF REFERENCES. Enter the total number of teferences cited in the report.

8s. CONTRACT OR GRANT NUMBER: If appropriate, enter the applicable number of the contract or grant under which the teport was written

$8 b, 8 c, \& 8 d$. PROJECT NUMBER: Enter the appropriate military department identification, such as project number. subproject number, system numbers, task number, etc.

9a. ORIGINATOR'S REPORT NUMBER(S): Enter the official report number by which the document will be identified and controlled by the originating activity. This number must be unique to this report.

9b. OTHER REPORT NUMBER(S): If the report has been assigned any other report numbers (either by the orisinator or by the sponsor), al so enter this number(s).
10. AVAILABILITY/LIMITATION NOTICES: Enter any limitations on further dissemination of the report, other than those imposed by security classification, using standard statements such as:

(1) "Qualified requesters may obtain copies of this report from DDC"

(2) "Foreign announcement and dissemination of this report by DDC is not authorized."

(3) "U. S. Government agencies may obtain copies of this report directiy from DDC. Other qualified DDC users shall request through

(4) "U. S. military agencies may obtain copies of this report directly from DDC. Other qualified users shall request through

."

(5) "All distribution of this report is controlled. Quatified DDC users shall request through ,

If the report has been furnished to the Office of Technical Services, Department of Commerce, for sale to the public, indicate this fact and enter the price, if known.

11. SUPPLEMENTARY NOTES: Use for additional explanatory notes.

12. SPONSORING MILITARY ACTIVITY: Enter the name of the departmental project office or laboratory sponsoring (pay ing for) the research and development. Include address.

13. ABSTRACT: Enter an abstract giving a brief and factual summary of the document indicative of the report, even though it may also appear elsewhere in the body of the technical report. If additional space is required, a continuation sheet shall be attached.

It is highly desirable that the abstract of classified reports be unclassified. Each paragraph of the abstract shall end with an indication of the military security classification of the information in the paragraph, represented as (TS), (S), (C), or $(U)$

There is no limitation on the length of the abstract. However, the suggester length is from 150 to 225 words.

14. KEY WORDS: Key words are technically meaningful terms or short phrases that characterize a report and may be used as index entries for cataloging the report. Key words must be selected so that no security classification is required. Idenselected so that no security classification is required. tary project code name, geopraphic location, may be used as key words but will be followed by an indication of technical context. The assignment of links, rules, and weights is optional. 



Metz Reference Roo: University of III
B106 NCEI

B106 NCEI Street

208 N. Rominois 61801

Uurbana. II 